\title{
Derived Colourings of Graphs
}

\author{
by
}

\section{Benjamin D. Seamone}

A thesis submitted to the Faculty of Graduate and Postdoctoral Affairs in partial fulfillment of the requirements for the degree of

\author{
Doctor of Philosophy \\ in \\ Applied Mathematics \\ Carleton University \\ Ottawa, Ontario, Canada
}

(C) 2012

Benjamin D. Seamone 
Library and Archives

Canada

Published Heritage

Branch

395 Wellington Street

Ottawa ON K1A ON4

Canada
Bibliothèque et

Archives Canada

Direction du

Patrimoine de l'édition

395 , rue Wellington

Ottawa ON K1A ON4

Canada
Your file Votre référence

ISBN: 978-0-494-93691-7

Our file Notre référence

ISBN: $978-0-494-93691-7$
NOTICE:

The author has granted a nonexclusive license allowing Library and Archives Canada to reproduce, publish, archive, preserve, conserve, communicate to the public by telecommunication or on the Internet, loan, distrbute and sell theses worldwide, for commercial or noncommercial purposes, in microform, paper, electronic and/or any other formats.

The author retains copyright ownership and moral rights in this thesis. Neither the thesis nor substantial extracts from it may be printed or otherwise reproduced without the author's permission.
AVIS:

L'auteur a accordé une licence non exclusive permettant à la Bibliothèque et Archives Canada de reproduire, publier, archiver, sauvegarder, conserver, transmettre au public par télécommunication ou par l'Internet, prêter, distribuer et vendre des thèses partout dans le monde, à des fins commerciales ou autres, sur support microforme, papier, électronique et/ou autres formats.

L'auteur conserve la propriété du droit d'auteur et des droits moraux qui protege cette thèse. $\mathrm{Ni}$ la thèse ni des extraits substantiels de celle-ci ne doivent être imprimés ou autrement reproduits sans son autorisation.
In compliance with the Canadian Privacy Act some supporting forms may have been removed from this thesis.

While these forms may be included in the document page count, their removal does not represent any loss of content from the thesis.
Conformément à la loi canadienne sur la protection de la vie privée, quelques formulaires secondaires ont été enlevés de cette thèse.

Bien que ces formulaires aient inclus dans la pagination, il n'y aura aucun contenu manquant. 


\section{Abstract}

The 1-2-3 Conjecture, posed by Karonski, Luzcak, and Thomason in 2004, states that any connected graph on at least three vertices can be edge-weighted from $\{1,2,3\}$ so that any two adjacent vertices have differing sums of weights from their respective incident edges. The 1-2-3 Conjecture, which is motivated by the study of irregularity strength, is open to a wide variety of variations. This thesis explores the following general problem:

"What is the fewest number of weights required to induce a particular type of colouring of a graph by some operation on the weights?"

This general problem lends itself to a variety of approaches; those used here include constructive methods, algebraic methods, probability theory, and graph homomorphisms.

In Chapter 2, we summarize the progress that has been made toward solving the 1-2-3 Conjecture, as well as the many interesting problems that it has inspired. In Chapter 3 , the focus is on classifying graphs for which two edge weights may be used to properly colour vertices by sums. Chapter 4 focuses on list-weightings. Using the Combinatorial Nullstellensatz, we prove the first non-trivial bounds for the maximum list size which guarantees an edge weighting which properly colours vertices by sums; 
these methods are extended to total weightings and colouring by products. Proper colourings of vertices obtained from vertex list-weightings are also considered. In Chapter 5 , we apply the methods of the previous chapter to solve the colouring by sums problem for digraphs. In Chapter 6 , the first study of weightings that properly colour vertices by sequences is undertaken. Using probabilistic methods, a variety of results are proven on the sequence variations of the 1-2-3 Conjecture and irregularity strength. Chapter 7 focuses on a new colouring problem. Given an edge weighting, we require that the multisets of colours seen by adjacent vertices are as different as possible. This problem has ties to injective colourings and fractional colourings; upper and lower bounds are proven on the number of weights needed for a given graph. 


\section{Acknowledgments}

Thank you to my supervisor, Brett Stevens. Your unwavering belief in my abilities was constant motivation. You are a model of the academic I strive to become.

Thank you to Prosenjit Bose. You saw me as a research colleague when I still saw

myself as a student. I am grateful for the opportunities that have arisen because of your interest in working with me.

Thank you to Mike Newman, Reza Naserasr, and Mahdad Khatirinejad for the helpful (and sometimes lively) discussions on the 1-2-3 Conjecture.

Thank you to the members of my examination committee: Mike Newman, Jason Gao, Mateja Šajna, Prosenjit Bose, and Roger Yu. Your comments and suggestions improved the final presentation of this work.

Thank you to the Natural Sciences and Engineering Research Council of Canada, le Fonds de recherche du Québec - nature et technologies, and Carleton University for their financial support.

Thank you to my parents, Laura Lee and Gary, and my brother, Luke. You are the reason I am who I am today. You are always loved and appreciated.

Thank you to my wife, Jen. It is a testament to your patience and self-giving that this thesis exists. Thank you so much for the motivation and the love. I could never have done it without you. 
To my son, Elliott - thank you for being you. My life is immeasurably better because you are here.

To everyone else who has had a positive influence on my life - you are too numerous to continue to acknowledge by name. Thank you. I am blessed. 
Dedicated to my family. 


\section{Table of Contents}

$\begin{array}{ll}\text { Abstract } & \text { ii }\end{array}$

Acknowledgments $\quad$ iv

Table of Contents vii

List of Tables $\quad$ x

List of Figures $\quad$ xi

1 Definitions and Notation 1

1.1 Graphs, multigraphs, and digraphs ................ 1

1.2 Walks, paths, and connectivity . . . . . . . . . . . . 3

1.3 Important graphs and subgraphs ............... 4

1.4 Graph functions . . . . . . . . . . . . . . 7

1.4 .1 Colourings .................... 7

1.4 .2 Weightings.................... 8

1.4.3 Colourings Derived from Weightings . . . . . . . . . . 10

2 Weights and Colours $\quad 11$ 
2.1 The $1-2-3$ Conjecture $\ldots \ldots \ldots \ldots \ldots \ldots$

2.2 Variation I: Colouring by products, multisets, and sets $\ldots \ldots \ldots .14$

2.3 Variation II: Total and vertex weightings $\ldots \ldots \ldots \ldots \ldots$

2.3 .1 Total weightings $\ldots \ldots \ldots \ldots \ldots \ldots$

2.3 .2 Vertex weightings . . . . . . . . . . . . . . 23

2.4 Variation III: List weightings . . . . . . . . . . . . . . 26

2.4 .1 Edge list-weightings . . . . . . . . . . . . . . 27

2.4 .2 Total list-weightings $\ldots \ldots \ldots \ldots \ldots \ldots \ldots$

2.4 .3 Vertex list-weightings . . . . . . . . . . . . . . . 33

2.5 Variation IV: Edge colourings $\ldots \ldots \ldots \ldots$

2.5 .1 Edge colourings . . . . . . . . . . . . . . . 34

2.6 Variation V: Distinguishing colourings $\ldots \ldots \ldots \ldots \ldots$

2.6.1 Distinguishing elements by edge-weightings . . . . . . 36

2.6.2 Distinguishing elements by total- and vertex-weightings . . . 38

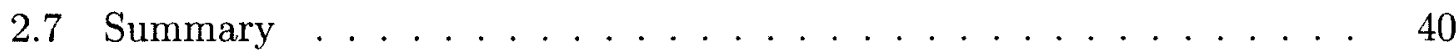

3 Edge 2-Weightings Which Colour Vertices by Sums 43

3.1 Preliminaries . . . . . . . . . . . . . . . 43

3.2 Building blocks: Weight colourings of basic graphs . . . . . . . . 46

3.3 Bipartite graphs . . . . . . . . . . . . . . . 65

3.4 Families of 2-weight colourable graphs $\ldots \ldots \ldots \ldots \ldots 72$

3.5 Graphs which are not 2-weight colourable . . . . . . . . 81

4 List Weightings Which Colour Graph Elements by Sums $\quad 86$

4.1 Polynomials, permanents, and the Combinatorial Nullstellensatz . . 87 
4.2 Bounds for edge list-weightings and total list-weightings . . . . . . . . 96

4.3 Colouring graph products by list-weightings . . . . . . . . . . 111

4.4 Additive colourings from list-weightings . . . . . . . . . . . . 115

5 Edge Weightings Which Colour Vertices in Digraphs by Sums 119

5.1 Main Result . . . . . . . . . . . . . . . . . 119

6 List Weightings Which Distinguish Vertices by Sequences 123

6.1 Introduction . . . . . . . . . . . . . . . . 123

6.2 Colouring by sequences for some $E(G)$ ordering . . . . . . . . . . . . 126

6.3 Colouring by sequences for any $E(G)$ ordering . . . . . . . . . . . . 131

6.4 Sequence irregularity strength . . . . . . . . . . . . . 140

7 Minimally Intersecting Multisets and Injective Colourings $\quad 146$

7.1 Motivation and definitions . . . . . . . . . . . . . 146

7.2 Relation to other edge colourings . . . . . . . . . . . . 149

7.3 A homomorphism approach ................. 151

7.4 Parameter values for classes of graphs . . . . . . . . . . . 158

8 Conclusion $\quad 166$

8.1 Summary and open problems . . . . . . . . . . . 167

List of References $\quad 182$ 


\section{List of Tables}

$1 \quad(k, l)$-weight choosability of classes of graphs . . . . . . . . . . 29

2 An edge 2-weighting that properly colours $V\left(C_{5}\right)$ by sequences . . . . 124

3 Summary of derived colouring parameter values . . . . . . . . . . . . 181 


\section{List of Figures}

1 Graphs having $\chi(G)=\Delta(G)$ with different values of $\chi_{\Sigma}^{e}(G) . \ldots . \quad 48$

2 An $\{a, b\}$-weight colouring of the Petersen graph . . . . . . . 73

3 Replacement operation to expand 2-weight colourings to larger cycles. $\quad 75$

$4 \quad$ An $\{a, b\}$-weight colouring of $K_{2} \square K_{n} \ldots \ldots \ldots \ldots$

$5 \quad$ An $\{a, b\}$-weight colouring of $K_{2} \square C_{7} \ldots \ldots \ldots 77$

6 Replacement operation for $\{a, b\}$-weight colourings of $K_{2} \square C_{2 k+1} \ldots \quad 78$

$7 \quad$ A partial edge $\{a, b\}$-weighting of $K_{2} \square C_{3} \ldots \ldots \ldots$

8 2-weight colourable graphs with forced weightings . . . . . . . 83

$9 \quad$ A digraph used to illustrate $A_{D}, B_{D}$, and $M_{D} \ldots \ldots . \ldots 9$

10 The associated matrices of $D$ from Figure $9 \ldots \ldots$. . . . . . . . 93

11 The block matrix $Y \ldots \ldots \ldots \ldots$. . . . . . . . . . . . 98

12 The matrices $Y^{\prime}$ and $Z^{\prime} \ldots \ldots \ldots \ldots$. . . . . . . . . . . . . .

13 An orientation of $K_{3}$ with a leaf . . . . . . . . . 101

14 An orientation of $C_{4}$ with a chord . . . . . . . . . . 102

15 The induced 2-path $u v w$ in $G \ldots \ldots 103$

16 An orientation $D$ of a graph $G$ with an induced 2-path uvw $\ldots . .105$

17 An operation on two columns of $A_{D} \ldots \ldots \ldots 5$

18 A labelled 5 -cycle . . . . . . . . . . . . . . . . . 124 
19 Two types of alternating 3-walks . . . . . . . . . . . . 154

20 An edge weighting showing that $\chi_{n}^{e}\left(C_{4 n} \square C_{4 m}\right)>3 \ldots \ldots 163$

21 A planar graph with $\chi_{n}^{e}(G)=8 \ldots \ldots \ldots \ldots$. . . . . . . . 177 


\section{Chapter 1}

\section{Definitions and Notation}

Standard graph theory notation and terminology will be used (see [20], [38]) but, for clarity, some terms and notation are explicitly defined here. Terminology required only in particular sections of this thesis will be defined as needed.

\subsection{Graphs, multigraphs, and digraphs}

A graph $G$ is a pair of sets $(V(G), E(G))$, often written $(V, E)$, where $V(G)$ is an arbitrary set, called the vertex set of $G$, and $E(G)$ is a set of distinct unordered pairs of distinct elements from $V(G)$, called the edge set of $G$. An edge $\{u, v\}$ is frequently written $u v$ for convenience. The collection of vertices and edges of a graph is called the set of elements of the graph. Two vertices $u, v \in V(G)$ are said adjacent in $G$ if $u v \in E(G)$, and two adjacent vertices are said to be neighbours. The set of neighbours of $v \in V(G)$ is called the (open) neighbourhood of $v$, and is denoted $N_{G}(v)$. The closed neighbourhood of $v \in V(G)$ is $N_{G}[v]=N_{G}(v) \cup\{v\}$. Two distinct edges $e, f \in E(G)$ are adjacent if there exists a vertex $v \in V(G)$ such that $v \in e \cap f$. A vertex $v \in V(G)$ and an edge $e \in E(G)$ are incident if $v \in e$. 
The degree of a vertex $v \in V(G)$ is the number of edges to which it is incident (equivalently, the size of $N_{G}(v)$ ), and is denoted $d_{G}(v)$ or simply $d(v)$ when $G$ is clear from context. The minimum and maximum degree of a vertex in $G$ are denoted $\delta(G)$ and $\Delta(G)$. A leaf of a graph is a vertex of degree 1. A graph is regular if every vertex has the same degree; if every vertex $v \in V(G)$ has degree $d_{G}(v)=d$, then we say $G$ is $d$-regular. A vertex is universal if it is adjacent to every other vertex in the graph. Two vertices $u, v \in V(G)$ are called twins if $N_{G}(u) \backslash\{v\}=N_{G}(v) \backslash\{u\}$, and are specifically called adjacent twins if they are twins and $u v \in E(G)$.

A multigraph $M$ is a pair $(V(M), E(M))$ defined similarly to a graph, however one no longer requires than an edge be a pair of distinct vertices (i.e. an edge may be a loop incident to a single vertex) nor do all pairs need to be distinct (i.e. multiple edges are allowed between vertices and multiple loops may be incident to a vertex). Two or more edges between the same pair of vertices are said to be parallel. The maximum edge multiplicity of a multigraph $M$ is the size of the largest set of parallel edges and is denoted $\mu(M)$. Note that graphs are occasionally called simple graphs to emphasize that they are not multigraphs.

A directed graph (or, digraph) $D$ is a pair $(V(D), A(D))$ where $V(D)$ is an arbitrary set and $A(D)$ is a set of ordered pairs of vertices, called the $\operatorname{arcs}$ of $D$. For an arc $a=(u, v) \in A(D), u$ is called the tail of $a$ and $v$ is called the head of $a$. Note that, unless otherwise stated, digraphs are permitted to have parallel arcs (arcs whose heads and tails are equal) and loops. The underlying graph of a digraph $D$ is the graph with vertex set $V(D)$ and an edge connecting any two vertices which appear in an arc of $D$. An orientation of a graph $G$ is a directed graph $D$ whose underlying graph is $G$. The indegree of a vertex in a digraph $v \in V(D)$ is the 
number of arcs having $v$ as their head and the outdegree of a vertex in a digraph $D$ is the number of arcs having $v$ as their tail; these two parameters are denoted $d^{-}(v)$ and $d^{+}(v)$, respectively. The weighted degree of a vertex $v \in V(D)$ is defined to be $d_{H}(v)=d_{H}^{+}(v)-d_{H}^{-}(v)$. A digraph $D$ is Eulerian if $d_{D}^{-}(v)=d_{D}^{+}(v)$ for every vertex $v \in V(D)$ (in other words, every vertex has weighted degree 0 ).

\subsection{Walks, paths, and connectivity}

A walk in a graph $G$ is a finite (ordered) sequence of vertices $W=v_{0}, v_{1}, \ldots, v_{t}$ (not necessarily distinct) such that $v_{i}$ is adjacent to $v_{i+1}$ for $0 \leq i \leq t-1$; for ease of notation, walks are frequently denoted $W=v_{0} v_{1} \cdots v_{t}$ and such a walk is said to have length $t$. A walk $W$ may also be considered as an ordered sequence of edges $\left\{v_{0} v_{1}, v_{1} v_{2}, \ldots, v_{t-1} v_{t}\right\}$; the length of $W$ is equal to the length of this edge sequence. The ends of a walk $W=v_{0} v_{1} \cdots v_{t}$ are the vertices $v_{0}, v_{t}$; the remaining vertices are called internal vertices.

A path is a walk where all vertices are distinct, and a $u v$-path is a path whose ends are $u, v \in V(G)$. Note that the length of a path is precisely the number of edges it contains. The distance between two vertices $u, v \in V(G)$, denoted $d_{G}(u, v)$, is the length of a shortest $u v$-path. The distance between two edges $u v, x y \in E(G)$ is the minimum of $\left\{d_{G}(u, x), d_{G}(u, y), d_{G}(v, x), d_{G}(v, y)\right\}$. The diameter of $G$, denoted $\operatorname{diam}(G)$, is the maximum distance between any two vertices of $G$.

Two paths are called internally disjoint if they share no internal vertices. A graph is $k$-connected if there exist at least $k$ pairwise internally disjoint paths between any pair of vertices. In particular, a graph is connected if there exists a path 
between any pair of vertices. A component of a graph is a maximal connected subgraph with respect to subgraph containment. A cut vertex of a graph is one whose removal (and hence the removal of all incident edges) increases the number of components in the graph. Note that a graph on at least 3 vertices is 2-connected if and only if it has no cut vertex. A separation of a connected graph is a decomposition of the graph into two nontrivial subgraphs (subgraphs containing at least one edge) which have precisely one vertex in common; the common vertex is called a separating vertex of the graph. In the case of a loopless graph, a separating vertex is precisely a cut vertex. A graph is nonseparable if it has no separating vertices and separable otherwise. Nonseparable graphs on at least 3 vertices are 2-connected, and any 2-connected (loopless) graph is nonseparable. A maximal nonseparable subgraph of $G$ is a block of $G$; a block is isomorphic either to $K_{2}$ or to a 2-connected graph. An end block of $G$ is a block which contains at most one separating vertex of $G$.

\subsection{Important graphs and subgraphs}

Let $k \in Z^{+}$and $m, n, n_{1}, n_{2}, \ldots, n_{k} \in Z^{+}$. Below are the definitions of some commonly used graphs:

- $P_{n}$ - a path with $n$ edges (and hence $n+1$ vertices);

- $C_{n}$ - a cycle with $n$ edges (and hence $n$ vertices), or the graph with $V\left(C_{n}\right)=\left\{v_{i}: 1 \leq i \leq n\right\}$ and $E\left(C_{n}\right)=\left\{v_{1} v_{2}, v_{2} v_{3}, \ldots, v_{n-1} v_{n}, v_{n} v_{1}\right\}$

- $K_{n}$ - a complete graph on $n$ vertices, or the graph on $n$ vertices where every pair of vertices is connected by an edge; 
- $K_{m, n}$ - a complete bipartite graph with parts of size $m$ and $n$, or the graph with $V\left(K_{m, n}\right)=A \cup B, A \cap B=\emptyset,|A|=m$, and $|B|=n$, and $E\left(K_{m, n}\right)=\{a b: a \in A, b \in B\}$;

- $K_{n_{1}, \ldots, n_{k}}$ - complete $k$-partite graph, or the graph on the vertex set $V(G)=A_{1} \cup \cdots \cup A_{k}$ (where $\left|A_{i}\right|=n_{i}$ for each $i=1, \ldots, k$ and $A_{i} \cap A_{j}=\emptyset$ for any $i \neq j$ ) and edge set $\left\{a_{i} a_{j}: a_{i} \in A_{i}, a_{j} \in A_{j}, i \neq j\right\}$;

- $\Theta_{n_{1}, \ldots, n_{k}}$ - generalized theta graph, or the graph obtained by taking two vertices, say $x$ and $y$, and connecting them with $k$ pairwise internally disjoint $x y$-paths of lengths $n_{1}, \ldots, n_{k}$;

- $W_{n}$ - $n$-wheel, or the graph obtained from adding a universal vertex to a cycle on $n$ vertices, $C_{n}$;

- $L(G)$ - the line graph of a graph $G$, or the graph with vertex set $E(G)$ where two vertices in $L(G)$ are adjacent if they are adjacent edges in $G$;

- $G \square H$ - the Cartesian product of two graphs $G$ and $H$, or the graph having vertex set $V(G) \times V(H)$ where two vertices $\left(u, u^{\prime}\right)$ and $\left(v, v^{\prime}\right)$ are adjacent if and only if either $u=v$ and $u^{\prime}$ is adjacent to $v^{\prime}$ in $H$ or $u^{\prime}=v^{\prime}$ and $u$ is adjacent to $v$ in $G$.

A graph $H$ is subgraph of a graph $G$ if $V(H) \subseteq V(G)$ and $E(H) \subseteq E(G)$. A spanning subgraph $H$ of $G$ is a subgraph with $V(H)=V(G)$. The subgraph of $G$ obtained by deleting a set of edges $A \subseteq E(G)$ is denoted $G-A$. The subgraph obtained by deleting a set of vertices $X \subseteq V(G)$ (and, hence, also deleting all edges incident to vertices in $X$ ) is similarly denoted $G-X$. For a subset of vertices 
$X \subseteq V(G)$, the subgraph $G-(V(G) \backslash X)$ is denoted $G[X]$ and is called the subgraph of $G$ induced by $X$. An induced subgraph $G[X]$ may also be viewed as the graph obtained by taking $X$ as the vertex set and the set of all edges of $G$ which have both ends in $X$ as the edge set. The graph obtained from $G$ by contracting an edge $u v=e \in E(G)$, denoted $G / e$, is the graph obtained by adding a vertex $x$ to $G-\{u, v\}$ with $x$ adjacent to precisely those vertices in $N_{G}(u) \cup N_{G}(v) \backslash\{u, v\}$. If $U \subseteq E(G)$, then the graph $G / U$ is obtained by iteratively contracting the edges in $U$; alternately, if $X \subseteq V(G)$ is the set of ends of the edges of $U$, then $G / U$ is obtained by adding a vertex $x$ to $G-X$, with $x$ adjacent to precisely those vertices in $\cup_{v \in X} N_{G}(v) \backslash X$.

A $t$-factor of a graph $G$ is a spanning subgraph with each vertex having degree $t$. The core ${ }^{1}$ of a graph $G$ is the subgraph obtained by repeated deletion of vertices of degree 1 until none remain. A tree is a connected graph containing no cycle as a subgraph. A forest is a graph where every connected component is a tree. A unicyclic graph is a graph which contains exactly one cycle as a subgraph. The girth of a graph is the length of its shortest cycle; the girth of a tree is assumed to be 0 . A chord of a cycle $C$ in a graph $G$ is an edge $e \in E(G)-E(C)$ whose ends lie on $C$.

If the vertices of a set $X \subseteq V(G)$ are pairwise non-adjacent, then $X$ is called an independent set or stable set of the graph $G$. A clique in a graph $G$ is a set of vertices $X \subseteq V(G)$ such that $G[X]$ is isomorphic to a complete graph. The clique number of a graph $G$, denoted $\omega(G)$, is the size of a largest clique in $G$.

\footnotetext{
${ }^{1}$ The core of a graph has multiple standard definitions, including one that is fundamental to the study of graph homomorphisms; we will not require other such definitions.
} 


\subsection{Graph functions}

The focus of this thesis is the interplay of functions on graphs. There are three basic types of graph functions:

- $f: V(G) \rightarrow S$ (vertex function)

- $f: E(G) \rightarrow S$ (edge function)

- $f: V(G) \cup E(G) \rightarrow S$ (total function).

Graph functions may go by a variety of names, two of the most common being colourings and weightings. The choice of name depends solely on the purpose of the function, described below.

\subsubsection{Colourings}

A colouring is a graph function whose purpose is to distinguish objects in the graph from one another according to some specified rule. The following types of colourings play a role in the work that follows:

- a proper colouring of $G$ is a graph function $c$ such that $c(a) \neq c(b)$ for any $a, b$ in the domain of $c$ which are adjacent or incident in $G$;

- a distinguishing colouring is a graph function $c$ such that $c(a) \neq c(b)$ for any distinct $a, b$ in the domain of $c$;

- a $t$-distance vertex colouring is a function $c: V(G) \rightarrow S$ such that $c(u) \neq c(v)$ for any $u, v \in V(G)$ such that $d(u, v) \leq t$, and a $t$-distance edge colouring of $G$ is a $t$-distance vertex colouring of $L(G)$; 
- an acyclic colouring of $G$ is a vertex colouring such that the subgraph induced by any two colour classes is a forest.

Proper colourings are of particular interest. We denote by $[n]$ the set $\{1,2, \ldots, n\}$. A graph $G$ is $k$-colourable (or edge $k$-colourable, or totally $k$-colourable) if there exists a proper colouring $c: V(G) \rightarrow[k]$ (respectively, $c: E(G) \rightarrow[k]$ or $c: V(G) \cup E(G) \rightarrow[k])$. The smallest values of $k$ for which a graph $G$ is $k$-colourable, edge $k$-colourable, totally $k$-colourable are called the chromatic number $(\chi(G))$, chromatic index $\left(\chi^{\prime}(G)\right)$, and total chromatic number $\left(\chi^{\prime \prime}(G)\right)$ of $G$, respectively. The smallest $k$ such that $G$ has an acyclic colouring with $k$ colours is called the acyclic chromatic number of $G$, which is denoted $A(G)$.

If each vertex $v$ is given a list of potential colours $L_{v}$, then a list colouring of $V(G)$ is an assignment $c(v) \in L_{v}$ for each $v \in V(G)$. A graph is $k$-choosable if there exists a proper list colouring for any assignment of lists of size $k$. The smallest $k$ such that $G$ is $k$-choosable is the choosability of $G$ (or list chromatic number of $G$ ), denoted $\operatorname{ch}(G)$. The list chromatic index and total list chromatic number are similarly defined for edge and total colourings, and are denoted $\operatorname{ch}^{\prime}(G)$ and $\operatorname{ch}^{\prime \prime}(G)$, respectively.

\subsubsection{Weightings}

A weighting (sometimes also called a labelling) of a graph is a function which is to be used for some other purpose or calculation. In this thesis, we will be particularly interested in ways in which weightings induce different types of colourings.

Given a set $S$, an edge $S$-weighting of $G$ is a weighting function $w: E(G) \rightarrow S$. A vertex $S$-weighting and total $S$-weighting are similarly defined. For sets $R$ 
and $S$, a total $(R, S)$-weighting of $G$ is a weighting $w: V(G) \cup E(G) \rightarrow R \cup S$ such that $w(v) \in R$ and $w(e) \in S$ for each $v \in V(G)$ and $e \in E(G)$. While it may sometimes be of interest to weight vertices or edges with elements from an arbitrary monoid, group, or field, we typically weight a graph with elements from $\mathbb{R}$.

We will often wish to distinguish the size of the image of a weighting function. Let $G$ be a graph and $k, r, s \in \mathbb{Z}^{+}$. A edge $k$-weighting, vertex $k$ weighting, or total $k$-weighting of $G$ is a weighting $w$ from $E(G), V(G)$, or $V(G) \cup E(G)$, respectively, into the set $[k]$. A total $(r, s)$-weighting of $G$ is a weighting $w: V(G) \cup E(G) \rightarrow\{1, \ldots, \max (r, s)\}$ such that $w(v) \leq r$ and $w(e) \leq s$ for each $v \in V(G)$ and $e \in E(G)$.

Finally, just as $k$-colourings may be generalized to list colourings, so too may the concept of $k$-weightings be extended to list weightings. Let $G$ be a graph and $k, r, s \in \mathbb{Z}^{+}$. Assign to each edge $e \in E(G)$ a list of weights $L_{e}$ and to each vertex $v$ a list of weights $L_{v}$. Let $\mathcal{E}=\cup_{e \in E(G)} L_{e}, \mathcal{V}=\cup_{v \in V(G)} L_{v}$, and $\mathcal{L}=\mathcal{E} \cup \mathcal{V}$. An edge list-weighting of $G$ is a function $w: E(G) \rightarrow \mathcal{E}$ such that $w(e) \in L_{e}$ for each $e \in E(G)$, a vertex list-weighting of $G$ is a function $w: V(G) \rightarrow \mathcal{V}$ such that $w(v) \in L_{v}$ for each $v \in V(G)$, and a total list-weighting of $G$ is a function $w: E(G) \cup V(G) \rightarrow \mathcal{L}$ such that $w(e) \in L_{e}$ for each $e \in E(G)$ and $w(v) \in L_{v}$ for each $v \in V(G)$. If the size of each list is at most $k$, then each weighting is referred to as an edge $k$-list-weighting, vertex $k$-list-weighting, or total $k$-list-weighting, respectively. If a total list-weighting has $\left|L_{v}\right| \leq r$ and $\left|L_{e}\right| \leq s$ for each $L_{v}$ and $L_{e}$, then the weighting is called a total $(r, s)$-list-weighting. 


\subsubsection{Colourings Derived from Weightings}

This thesis is concerned with the following general question:

Under what conditions does a graph weighting induce a particular type of graph colouring?

To illustrate, consider one of the earliest problems of this type, that of finding a graph's irregularity strength. Let $w: E(G) \rightarrow[k]$ be an edge weighting of a graph $G$. Define the weighted degree of a vertex $v$ to be $d_{G, w}(v)=\sum_{e \ni v} w(e)$. If the function $d_{G, w}$ is a vertex-distinguishing colouring (that is, $d_{G, w}(u) \neq d_{G, w}(v)$ for any distinct $u, v \in V(G)$ ), then $w$ is called an irregular assignment of $G$. The smallest value of $k$ for which a graph $G$ has an irregular assignment $w$ is called the irregularity strength of $G$. Irregularity strength was introduced by Chartrand et al. [29], motivated by a question regarding multigraphs. It is a well known fact that any simple graph $G$ must contain two vertices of the same degree. A multigraph need not have this property. A multigraph is called irregular if ay two distinct vertices have distinct degrees. "Let $M$ be a multigraph obtained from a simple graph $G$ by replacing an edge of $G$ with at most $k$ parallel edges. For a given $G$, what is the smallest such $k$ such that $M$ is irregular?" This value of $k$ is precisely the irregularity strength of $G$.

In the following chapter, we will survey a wide variety of colourings derived from weightings and their corresponding parameters, summarizing what is known about the possible values of these parameters and, where possible, illustrating relationships between them. 


\section{Chapter 2}

\section{Weights and Colours}

\subsection{The 1-2-3 Conjecture}

Much of the work on proper colourings derived from weightings is motivated by the following conjecture due to Karoński, Luczak, and Thomason [64]:

If $G$ is a graph without a component isomorphic to $K_{2}$, then it has an edge 3-weighting such any two adjacent vertices have distinct sums of incident edge weights.

Note that this conjecture relates closely to graph irregularity strength, explained at the end of the previous chapter. An edge 3-weighting corresponds to a multigraph with maximum edge multiplicity 3 , and so we are looking for a multigraph with adjacent vertices having distinct weighted degrees rather than every pair of vertices. If an edge $k$-weighting gives rise to such a proper vertex colouring, the weighting is a proper vertex colouring by sums. Denote by $\chi_{\Sigma}^{e}(G)$ the smallest value of $k$ such that a graph $G$ has a edge $k$-weighting which is a proper vertex colouring by sums. The notation $\chi_{\Sigma}^{e}(G)$ is a modification of notation proposed by Györi and 
Palmer [51]; there is no standard notation that is consistent across the literature on the subject. Note the three components - " $\chi$ " indicates that we desire a proper vertex colouring, the superscript " $e$ " indicates that the weighting of interest is of the edges, and the subscript " $\Sigma$ " indicates that vertex colours are obtained by adding up edge weights. As new parameters are defined for different combinations of weightings and colourings, we will develop notation consistent with this format.

A graph $G$ is nice if no connected component is isomorphic to $K_{2}$. Karoński, Euczak, and Thomason's conjecture, usually referred to as the 1-2-3 Conjecture, may be succinctly stated as follows:

1-2-3 Conjecture (Karoński, Luczak, Thomason [64]). If $G$ is nice, then $\chi_{\Sigma}^{e}(G) \leq 3$.

Early approaches to the 1-2-3 Conjecture focused on relating $\chi_{\Sigma}^{e}(G)$ to values of $k$ for which $G$ is $k$-colourable, including the following notable result from the authors of the conjecture:

Theorem 2.1.1 (Karoński, Łuczak, Thomason [64]). If $(\Gamma,+)$ is a finite abelian group of odd order and $G$ is a nice $|\Gamma|$-colourable graph, then there is a weighting of the edges of $G$ with the elements of $\Gamma$ such that the vertex colouring by sums is a proper vertex colouring.

In particular, if $G$ is nice and $k$-colourable for $k$ odd, then $\chi_{\Sigma}^{e}(G) \leq k$. This theorem was then extended to the following:

Theorem 2.1.2. If $G$ is 2-connected and $\chi(G) \geq 3$, then $\chi_{\Sigma}^{e}(G) \leq \chi(G)$. In particular, for any integer $k \geq 3$ and nice graph $G$, the following hold:

1. (Karoński, Luczak, Thomason [64]) if $G$ is $k$-colourable for $k$ odd, then $\chi_{\Sigma}^{e}(G) \leq k$ 
2. (Duan, Lu, Yu [40]) if $G$ is $k$-colourable for $k \equiv 0(\bmod 4)$, then $\chi_{\Sigma}^{e}(G) \leq k$;

3. (Duan, Lu, Yu [40]) if $\delta(G) \leq k-2$, then $\chi_{\Sigma}^{e}(G) \leq k$;

4. (Lu, Yang, Yu [69]) if $G$ is 2-connected, $k$-colourable, and has $\delta(G) \geq k+1$ for $k \equiv 2(\bmod 4)$, then $\chi_{\Sigma}^{e}(G) \leq k$.

It is also shown in [40] that, for a connected graph $G, \chi_{\Sigma}^{e}(G) \leq \chi(G)$ if $G$ has an odd number of vertices or if there is a proper $\chi(G)$-colouring where one colour class has even size.

The most significant progress toward solving the 1-2-3 Conjecture is the establishment and improvement of constant bounds on $\chi_{\Sigma}^{e}(G)$ for every nice graph $G$. This is the best known bound to date:

Theorem 2.1.3 (Kalkowski, Karo'nski, Pfender [62]). If $G$ is a nice graph, then $\chi_{\Sigma}^{e}(G) \leq 5$

Addario-Berry, Dalal, McDiarmid, Reed, and Thomason [2] previously showed that if $G$ is a nice graph, then $\chi_{\Sigma}^{e}(G) \leq 30$. This was improved to $\chi_{\Sigma}^{e}(G) \leq 16$ by Addario-Berry, Dalal, and Reed [3], then to $\chi_{\Sigma}^{e}(G) \leq 13$ by Wang and Yu [90], and then to $\chi_{\Sigma}^{e}(G) \leq 6$ by Kalkowski, Karoński, and Pfender [61].

It is easily seen that there exist nice graphs for which two edge weights do not suffice to colour the vertices by sums (e.g. $K_{3}, C_{6}$ ), and hence the best possible constant bound for all nice graphs is the conjectured value of 3 . However, it is known that, for any constant $p \in(0,1)$, if $G$ is a random graph chosen from $\mathcal{G}_{n, p}$, (that is, a graph with vertex set $[n]$ and, for each $1 \leq i<j \leq n$, an edge connects $i$ and $j$ with probability $p$ ), then asymptotically almost surely $\chi_{\Sigma}^{e}(G) \leq 2[3]$. It is also known that $\chi_{\Sigma}^{e}(G) \leq 2$ if $G$ lies in one of a few classes of graphs. Chang, Lu, Wu, and Yu 
[28] showed that $\chi_{\Sigma}^{e}(G) \leq 2$ for a variety of graph classes, notably if $G$ is bipartite and $d$-regular for $d \geq 3$. Lu, Yu, and Zhang [70] proved that if $G$ is a nice graph which is either 3-connected and bipartite or has minimum degree $\delta(G) \geq 8 \chi(G)$, then $\chi_{\Sigma}^{e}(G) \leq 2$. Recently, Jahanbekam and West [57] showed that $\chi_{\Sigma}^{e}(G) \leq 2$ if $G$ is $r$-regular with $\chi(G) \leq p \leq \frac{r+2}{2}$ for some odd prime $p$.

\subsection{Variation I: Colouring by products, multisets, and sets}

There are a variety of ways in which one may modify the way in which vertex colours are obtained from an edge weighting of a graph. The first variations we consider are those where addition of edge weights as the colouring method is replaced by another operation; in particular, we consider variations where colours are obtained by taking the product, multiset, or set of weights from edges incident to $v$ for each $v \in V(G)$. We will adopt the notation $\{\{\cdot\}\}$ for a multiset. If such a colouring is proper, then the edge $k$-weighting of $G$ is a proper vertex colouring by products, multisets or sets, respectively. The smallest $k$ for which such colourings exist for a graph $G$ are denoted $\chi_{\Pi}^{e}(G), \chi_{m}^{e}(G)$ and $\chi_{s}^{e}(G)$, respectively. The following proposition is used frequently in our study of colouring by multisets:

Proposition 2.2.1. If $\min \left\{\chi_{\Sigma}^{e}(G), \chi_{\Pi}^{e}(G), \chi_{s}^{e}(G)\right\} \leq k$, then $\chi_{m}^{e}(G) \leq k$.

The first bound on $\chi_{m}^{e}(G)$ was established in [64], where Karoński, Luczak, and Thomason showed that if $G$ is a nice graph, then $\chi_{m}^{e}(G) \leq 183$. It follows from the 1-2-3 Conjecture and Proposition 2.2.1 that the expected upper bound for $\chi_{m}^{e}(G)$ is 
3 ; whether or not $\chi_{m}^{e}(G) \leq 3$ remains open. However, it is known that $\chi_{m}^{e}(G) \leq 4$ for any nice graph $G$ :

Theorem 2.2.2 (Addario-Berry, Aldred, Dalal, Reed [1]). Let $G$ be a connected graph which is not 3-colourable. There exists a partition of $V(G)$ into sets $V_{0}, V_{1}, V_{2}$ such that there exists a weighting $w: E(G) \rightarrow\left\{c_{0}, c_{1}, c_{2}, c^{*}\right\}$ with the following properties

1. for each $i \in\{0,1,2\}$, every $v \in V_{i}$ is incident to at least one edge weighted $c_{i}$;

2. for each $i \in\{0,1,2\}$, the vertices in $V_{i}$ are incident only to edges weighted $c_{i}, c_{i-1(\bmod 3)}, c^{*}$, and

3. for each $i \in\{0,1,2\}$, if $u, v \in V_{i}$ are adjacent, then the number of edges incident to $u$ with weight $c_{i}$ is different from the number of edges incident to $v$ with weight $c_{i}$.

Theorem 2.2.3 (Addario-Berry, Aldred, Dalal, Reed [1]). If $G$ is a nice graph, then $\chi_{m}^{e}(G) \leq 4$.

Proof. If $G$ is 3-colourable, then $\chi_{m}^{e}(G) \leq \chi_{\Sigma}^{e}(G) \leq 3$ by Theorem 2.1.1 and Proposition 2.2.1. Suppose $G$ is not 3-colourable, and consider the partition of $V(G)$ and weighting function $w$ guaranteed by Theorem 2.2.2. Let $c(v)$ denote the multiset of weights from edges incident to $v$. Let $u v \in E(G)$. If $u, v \in V_{i}$, then property 3 guarantees that $c(u)$ and $c(v)$ have distinct numbers of $c_{i}$ 's. If $u \in V_{i}$ and $v \in V_{i+1}(\bmod 3)$, then $c(u)$ contains only the weights $c_{i}, c_{i-1}(\bmod 3), c^{*}$ and $c(v)$ contains only the weights $c_{i}, c_{i+1}(\bmod 3), c^{*}$. Property 1 from Theorem 2.2 .2 guarantees that $c(v)$ contains at least one weight of $c_{i+1(\bmod 3)}$, and hence $c(u) \neq c(v)$. Hence $\chi_{m}^{e}(G) \leq 4$ if $G$ is not 3-colourable. 
Addario-Berry et al. also use Theorem 2.2.2 to prove the following theorem:

Theorem 2.2.4 (Addario-Berry, Aldred, Dalal, Reed [1]). If $G$ is nice and $\Delta(G) \geq 1000$, then $\chi_{m}^{e}(G) \leq 3$.

Note that a bound on $\chi_{m}^{e}(G)$ implies a constant bound on $\chi_{\Pi}^{e}(G)$. The following corollary to the work of Addario-Berry et al. was first noted in [82]:

Corollary 2.2.5. If $G$ is a nice graph, then $\chi_{\Pi}^{e}(G) \leq 5$.

Proof. If $G$ is 3-colourable, then $\chi_{m}^{e}(G) \leq 3$ by Theorem 2.1.1. Let $w$ be an edge 3weighting of $G$ which properly colours $V(G)$ by multisets, and $m$ the induced proper vertex colouring by multisets. Define $w^{\prime}: E(G) \rightarrow\{2,3,5\}$ by $w^{\prime}(e)=w(e)$ if $w(e) \in\{2,3\}$ and $w^{\prime}(e)=5$ if $w(e)=1$. Let $c(v)=\prod_{e \ni v} w^{\prime}(e)$ for each $v \in V(G)$. If $c(u)=c(v)$, then, since 2,3 , and 5 are pairwise relatively prime, $m(u)$ must be equal to $m(v)$. Since $m$ is proper, so is $c$.

If $G$ is not 3-colourable, then let $w: E(G) \rightarrow\left\{c_{0}, c_{1}, c_{2}, c^{*}\right\}$ be the edge weighting guaranteed by Theorem 2.2.2. Define a new edge weighting $w^{\prime}: E(G) \rightarrow\{1,2,3,5\}$ by $w^{\prime}(e)=2$ if $w(e)=c_{0}, w^{\prime}(e)=3$ if $w(e)=c_{1}, w^{\prime}(e)=5$ if $w(e)=c_{3}$, and $w^{\prime}(e)=1$ if $w(e)=c^{*}$. Theorem 2.2 .2 guarantees that, for any two adjacent vertices $u, v \in V(G)$, there is a weight $j \in\{2,3,5\}$ for which $u$ and $v$ are incident to different numbers of edges with weight $j$. Since 2,3 , and 5 are pairwise relatively prime, this implies that $\prod_{e \ni u} w^{\prime}(e) \neq \prod_{e \ni v} w^{\prime}(e)$ for any $u v \in E(G)$, and hence $w^{\prime}$ properly colours $V(G)$ by products.

While constant bounds exist for $\chi_{\Sigma}^{e}(G), \chi_{\Pi}^{e}(G)$ and $\chi_{m}^{e}(G)$, this is not possible for the parameter $\chi_{s}^{e}(G)$. To see this, note that an edge $k$-weighting allows at most $2^{k}-1$ possible vertex colours by sets and so the complete graph on $2^{k}$ vertices must 
have $\chi_{s}^{e}(G)>k$. In the study of colouring by sets, Györi and Palmer establish a link between $\chi_{s}^{e}(G)$ and a hypergraph induced by $G$. A hypergraph $H=(V(H), E(H))$ consists of a set of vertices $V(H)$ and a set of hyperedges $E(H)$, where a hyperedge is a subset of $V(H)$; note that a graph is precisely a hypergraph whose hyperedges have size exactly 2. A hypergraph is said to have Property B if there exists a colouring $c: V(H) \rightarrow\{1,2\}$ such that every hyperedge contains vertices of both colours; this concept has its roots in set theory and is due to Bernstein [19].

Theorem 2.2.6 (Győri, Palmer [51]). Let $G$ be a bipartite graph with bipartition of the vertices $V(G)=X \cup Y$. If $H$ is the hypergraph with $V(H)=Y$ and $E(H)=\left\{N_{G}(x): x \in X\right\}$, then $\chi_{s}^{e}(G)=2$ if and only if $H$ has Property $B$.

In [50], Győri, Hornák, Palmer, and Woźniak show that if $G$ is nice, then $\chi_{s}^{e}(G) \leq 2\left\lceil\log _{2} \chi(G)\right\rceil+1$, a bound which was subsequently refined.

Theorem 2.2.7 (Györi, Palmer [51]). If $G$ is nice, then $\chi_{s}^{e}(G)=\left\lceil\log _{2} \chi(G)\right\rceil+1$.

The parameter $\chi_{s}^{e}(G)$ is denoted $\operatorname{gndi}(G)$ in [50] and called the general neighbourhood distinguishing index. The motivation for the parameter comes from the study of the neighbourhood distinguishing index of a graph $G$, denoted $\operatorname{ndi}(G)$, which is the smallest integer $k$ such that $G$ has a proper edge $k$-colouring such that adjacent vertices have distinct sets of colours on their incident edges. This parameter is also known as the adjacent vertex distinguishing chromatic index, and the type of colouring is called an adjacent strong edge-colouring or 1-strong edge-colouring. The neighbourhood distinguishing index was first introduced by Liu, Wang and Zhang [68], who propose the following conjecture: 
Conjecture 2.2.8 (Liu, Wang, Zhang [68]). If $G \notin\left\{K_{2}, C_{5}\right\}$ and $G$ is connected, then $\Delta(G) \leq \operatorname{ndi}(G) \leq \Delta(G)+2$.

While the lower bound is trivially true, the upper bound appears to be difficult to prove. Here are a few known bounds for $\operatorname{ndi}(G)$ :

Theorem 2.2.9 (Balister, Györi, Lehel, Schelp [13]). Conjecture 2.2.8 holds if $G$ is bipartite or $\Delta(G) \leq 3$.

Theorem 2.2.10 (Balister, Györi, Lehel, Schelp [13]). If a graph $G$ is nice, then $\operatorname{ndi}(G) \leq \Delta(G)+O(\log \chi(G))$.

Theorem 2.2.11 (Hatami [55]). If a graph $G$ is nice and $\Delta(G)>10^{20}$, then $\operatorname{ndi}(G) \leq \Delta(G)+300$.

Recalling Theorem 2.1.1 of Karoński et al., one may consider the minimum $s$ such that, for any abelian group $(\Gamma,+)$ of order $s$, a graph $G$ has an edge weighting from $\Gamma$ which properly colours $V(G)$ by sums. This parameter, called the group sum chromatic number and denoted $\chi_{g}^{\Sigma}(G)$, was introduced by Anholcer and Cichacz [12], who prove the following theorem:

Theorem 2.2.12 (Anholcer, Cichacz [12]). If $G$ is a graph with no component having fewer than 3 vertices, then $\chi(G) \leq \chi_{g}^{\Sigma}(G) \leq \chi(G)+2$.

Furthermore, the authors give a complete characterization of which graphs have group sum chromatic number $\chi(G), \chi(G)+1$, and $\chi(G)+2$.

Finally, we present one further variation of neighbour-distinguishing edge weightings, introduced by Baril and Togni [14]. A proper $k$-tuple edge-colouring of a graph $G$ assigns a set of $k$ colours to each edge such that adjacent edges have disjoint 
colour sets. The vertex version of such a colouring was introduced by Stahl [84]. Let $S(v)$ denote the union of all sets of colours assigned to edges incident to $v \in V(G)$. If $S(u) \neq S(v)$ for each $u v \in E(G)$, then the edge colouring is a $k$-tuple neighbourdistinguishing colouring. Baril and Togni determine the smallest $k$ for which various classes of graphs have $k$-tuple neighbour-distinguishing colourings. They also make the following conjecture, which extends Conjecture 2.2 .8 to multigraphs:

Conjecture 2.2.13 (Baril, Togni [14]). If $G$ is a connected graph, $G \neq C_{5}$, with edge multiplicity $\mu(G)$ and maximum degree $\Delta(G)$, then $\operatorname{ndi}(G) \leq \Delta(G)+\mu(G)+1$.

\subsection{Variation II: Total and vertex weightings}

\subsubsection{Total weightings}

Recall that a total weighting of a graph $G$ is an assignment of a real number weight to each $e \in E(G)$ and each $v \in V(G)$. The concept of proper colourings induced by total $k$-weightings was introduced by Przybyło and Wozniak [76]. Given a total $k$-weighting of $G$, we consider vertex colourings obtained by taking either the sum, product, multiset, or set of weights taken from the edges incident to $v$ and from $v$ itself for each $v \in V(G)$. If such a colouring is proper, then the total $k$-weighting of $G$ is a proper vertex colouring by sums, products, multisets or sets, respectively. The smallest values of $k$ such that a proper colouring of each type exists for a graph $G$ are denoted $\chi_{\Sigma}^{t}(G), \chi_{\Pi}^{t}(G), \chi_{m}^{t}(G)$ and $\chi_{s}^{t}(G)$, respectively. Note that, while one must exclude graphs with connected components containing precisely one edge when considering edge weightings which properly colour vertices by sums, this exclusion is not necessary for total weightings. 
Proposition 2.3.1. If $G$ is any graph, then

1. (Przybyło, Wozniak [76]) $\chi_{\Sigma}^{t}(G) \leq \chi_{\Sigma}^{e}(G)$,

2. (Skowronek-Kaziów, $[82]) \chi_{\Pi}^{t}(G) \leq \chi_{\Pi}^{e}(G)$,

3. $\chi_{m}^{t}(G) \leq \chi_{m}^{e}(G)$,

4. $\chi_{s}^{t}(G) \leq\left\lceil\log _{2} \chi(G)\right\rceil+1=\chi_{s}^{e}(G)$.

Proof. 1. Suppose $\chi_{\Sigma}^{e}(G)=k$. Let $w: V(G) \rightarrow[k]$ be an edge weighting which properly colours $V(G)$ by sums. Define the total weighting $w_{t}: V(G) \cup E(G) \rightarrow[k]$ by $w_{t}(e)=w(e)$ for each $e \in E(G)$ and $w_{t}(v)=1$ for each $v \in V(G)$ and define $c(v)=w_{t}(v)+\sum_{e \ni v} w_{t}(e)=1+\sum_{e \ni v} w(e)$. Since $\sum_{e \ni u} w(e) \neq \sum_{e \ni v} w(e)$ for any $u v \in E(G), c(u) \neq c(v)$ as well and so $w_{t}$ is a total $k$-weighting which properly colours $V(G)$ by sums.

2. Apply the same argument as 1 , assigning each vertex the weight 1 .

3. Apply the same argument as 1 , assigning all vertices the same weight from $[k]$.

4. We may assume that $G$ is connected. If $|V(G)|=1$, then $\chi_{s}^{t}(G)=1$ and the stated bound is obtained. Assume $|V(G)| \geq 3$, and hence that $\chi(G) \geq 2$. Let $V(G)=\left\{v_{1}, \ldots, v_{n}\right\}$ and let $k=\left\lceil\log _{2} \chi(G)\right\rceil+1$. Construct a new graph $H$ with vertex set $V(H)=V(G) \cup\left\{u_{1}, \ldots, u_{n}\right\}$ and edges $E(H)=E(G) \cup\left\{v_{1} u_{1}, v_{2} u_{2}, \ldots, v_{n} u_{n}\right\}$. Since $\chi(H)=\chi(G)$ and $H$ is nice, Theorem 2.2 .7 implies that $H$ has an edge $k$-weighting which properly colours $V(H)$ by sets, say $w: V(H) \cup E(H) \rightarrow[k]$. Define the total weighting $w_{t}: V(G) \cup E(G) \rightarrow[k]$ as $w^{\prime}(e)=w(e)$ for each $e \in E(G)$ and 
$w^{\prime}\left(v_{i}\right)=w\left(v_{i} u_{i}\right)$ for each $v_{i} \in V(G)$. Since each $v \in V(G)$ will receive the same colour under $w^{\prime}$ as under $w, w^{\prime}$ is a total $k$-weighting which properly colours $V(G)$ by sets, and so $\chi_{s}^{t}(G) \leq k$. Hence, $\chi_{n}^{e}(G) \leq k$, and since $k$ is the smallest such $k$ for which such a homomorphism exists, $\chi_{n}^{e}(G)=k$.

In general, allowing vertex weights can lead to fewer weights being needed to obtain a proper vertex colouring of a graph. The following conjecture motivates the study of total weightings and vertex colouring by sums:

1-2 Conjecture (Przybyło, Wozniak [76]). For every graph $G, \chi_{\Sigma}^{t}(G) \leq 2$.

The best known bound, to date, comes from the following result on total $(k, l)$ weightings; recall that a total $(k, l)$-weighting $w$ assigns $w(v) \in[k]$ and $w(e) \in[l]$.

Theorem 2.3.2 (Kalkowski [60]). Every graph has a total (2,3)-weighting which is a proper vertex colouring by sums. As a consequence, $\chi_{\Sigma}^{t}(G) \leq 3$ for any graph $G$.

This theorem improves previous results from [76], where it is shown that $\chi_{\Sigma}^{t}(G) \leq \min \{\lfloor\chi(G) / 2\rfloor+1,11\}$ for any graph $G$, and from [75], where it is shown that $\chi_{\Sigma}^{t}(G) \leq 7$ if $G$ is regular. It is shown in [76] that the 1-2 Conjecture holds if $G$ is complete, 3-colourable, or 4-regular. The aforementioned result of Jahanbekam and West [57] that $\chi_{\Sigma}^{e}(G) \leq 2$ if $G$ is an $r$-regular graph with $\chi(G) \leq p \leq \frac{r+2}{2}$ for some odd prime $p$ implies that $\chi_{\Sigma}^{t}(G) \leq 2$ for the same graphs. Furthermore, they show that if $\chi(G) \leq p \leq \frac{\delta(G)+4}{2}$ for some odd prime $p$, then $\chi_{\Sigma}^{t}(G) \leq 2$; as a consequence, $\chi_{\Sigma}^{t}(G) \leq 2$ if $\chi(G) \leq \frac{\delta(G)+10}{2}$.

The problem of obtaining proper vertex colourings by products from total $k$ weightings has also been studied; the following conjecture is proposed:

Conjecture 2.3.3 (Skowronek-Kaziów [82]). For every graph $G, \chi_{\Pi}^{t}(G) \leq 2$. 
In [82], Conjecture 2.3.3 was verified for graphs which are 3-colourable or complete, and it was shown that $\chi_{\Pi}^{t}(G) \leq 3$ for every graph $G$.

By the discussion above, the following theorem summarizes the known bounds for total weightings which are proper vertex colourings:

Theorem 2.3.4. For every graph $G, \chi_{\Sigma}^{t}(G), \chi_{\Pi}^{t}(G)$ and $\chi_{m}^{t}(G)$ are all at most 3 .

On the topic of total weighting which properly colour vertices by sets, much more attention has been paid to weightings which are themselves proper total colourings. Recall that, in a proper total colouring of $G$, adjacent vertices, adjacent edges, and incident vertices and edges must all receive different colours. As an extension of the neighbourhood distinguishing index discussed in Section 2.2, the authors of [96] study proper total colourings which, considered as weightings, also properly colour $V(G)$ by sets (the set of weights consisting of the colour of the vertex itself as well as the colours on all incident edges). The fewest number of colours required for such a colouring to exist for a graph $G$ is called the adjacent vertex distinguishing total chromatic number, which is denoted $\chi_{a t}^{\prime}(G)$. Since $\chi_{a t}^{\prime}(G) \geq \chi^{\prime \prime}(G)$, it follows immediately that $\chi_{a t}^{\prime}(G) \geq \Delta(G)+1$ for any graph $G$. Conversely, it is conjectured that any graph can be so coloured using a constant number of colours greater than its maximum degree.

Conjecture 2.3.5 (Zhang, Chen, Li, Yao, Lu, Wang [96]). For any graph $G$, $\Delta(G)+1 \leq \chi_{a t}^{\prime}(G) \leq \Delta(G)+3$.

This conjecture is reminiscent of Conjecture 2.2 .8 on edge colourings and, more notably, the Total Colouring Conjecture.

Total Colouring Conjecture (Behzad [18] and Vizing [88]). For any graph $G$, $\chi^{\prime \prime}(G) \leq \Delta(G)+2$. 
The best known bound on a graph's total chromatic number is due to Molloy and Reed [72], who show that $\chi^{\prime \prime}(G) \leq \Delta(G)+10^{26}$. The Total Colouring Conjecture is notoriously difficult, which suggests that Conjecture 2.3 .5 may also be very difficult to solve. However, Coker and Johannson [35] have recently shown that there exists a universal constant $C$ such that $\chi_{a t}^{\prime}(G) \leq \Delta(G)+C$. Their proof relies on a probabilistic argument to show that $\chi_{a t}^{\prime}(G) \leq \chi^{\prime \prime}(G)+C^{\prime}$ for some other constant $C^{\prime}$, then invokes Molloy and Reed's aforementioned bound on $\chi^{\prime \prime}(G)$.

\subsubsection{Vertex weightings}

Having considered edge and total weightings, we now turn our attention to vertex weightings. We consider the usual four methods of obtaining a vertex colouring from a vertex $k$-weighting, taking either the sum, product, multiset, or set of weights from vertices adjacent to $v$ for each $v \in V(G)$. If such a colouring is proper, then the vertex $k$-weighting of $G$ is a proper vertex colouring by sums, products, multisets or sets, respectively. The smallest values of $k$ such that a colouring of each type exists for a graph $G$ are denoted $\chi_{\Sigma}^{v}(G), \chi_{\Pi}^{v}(G), \chi_{m}^{v}(G)$ and $\chi_{s}^{v}(G)$, respectively.

A vertex weighting of $G$ which is a proper vertex colouring by sums is also known as an additive colouring of $G$ (initially called a lucky labelling of $G$ ), studied in [36] by Czerwiński, Grytczuk, and Żelazny. The value $\chi_{\Sigma}^{v}(G)$ is called the additive colouring number of $G$ (denoted $\eta(G)$ in [36]). The following conjecture is proposed:

Additive Colouring Conjecture (Czerwiński, Grytczuk, Żelazny [36]). For every graph $G, \chi_{\Sigma}^{v}(G) \leq \chi(G)$.

As evidence for the conjecture, note the following bound on $\chi_{m}^{v}(G)$ : 
Proposition 2.3.6 (Czerwiński, Grytczuk, Żelazny [36]; Chartrand, Okamoto, Salehi, Zhang [32]). For any graph $G, \chi_{m}^{v}(G) \leq \chi(G)$.

Proof. Let $k=\chi(G)$ and let $c: V(G) \rightarrow[k]$ be a vertex weighting which is a proper vertex colouring. Let $m(u)$ denote the multiset of weights from the vertices in $N_{G}(u)$. If $u v \in E(G)$, then $c(u) \in m(v) \backslash m(u)$. Thus, $m(v) \neq m(u)$ for any $u v \in E(G)$ and so $c$ is a vertex weighting which properly colours $V(G)$ by sums.

Note that the proof above actually shows the following:

Proposition 2.3.7. For any graph $G, \chi_{s}^{v}(G) \leq \chi(G)$.

Furthermore, it is shown in [34] that, for any graph $G$, there exists a set $S_{G}$ of $\chi(G)$ integers such that there exists a vertex weighting $w: V(G) \rightarrow S_{G}$ which is an additive colouring. This result is further extended in [33], where it is shown that the size of the smallest set of reals such that $G$ has an additive colouring is exactly $\chi_{m}^{v}(G)$ for any connected graph $G$.

It is easily shown that no constant bound is possible for $\chi_{\Sigma}^{v}(G)$ by noting that $\chi_{\Sigma}^{v}\left(K_{n}\right)=n$ for any $n \geq 2$. In [21], it is shown that $\chi_{\Sigma}^{v}(G) \leq 468$ if $G$ is planar, $\chi_{\Sigma}^{v}(G) \leq 36$ if $G$ is planar and 3-colourable, and $\chi_{\Sigma}^{v}(G) \leq 4$ if $G$ is planar and has girth at least 13. Czerwiński et al. [36] prove a general upper bound on $\chi_{\Sigma}^{v}(G)$ in terms of the acyclic chromatic number of $G$, denoted $A(G)$.

Theorem 2.3.8 (Czerwiński, Grytczuk, Żelazny [36]). For every graph $G$, $\chi_{\Sigma}^{v}(G) \leq p_{1} \cdots p_{r}$ where $p_{i}$ denotes the $i^{\text {th }}$ odd prime number and $r=\left(\begin{array}{c}A(G) \\ 2\end{array}\right)$.

Unfortunately, the bound given by Theorem 2.3.8 is extraordinarily large. Some bounds are known for $A(G)$ when $\Delta(G)$ is small: 
Lemma 2.3.9. If $G$ is a graph with maximum degree $\Delta(G)$, then

1. (Grünbaum [49]) $A(G) \leq 4$ if $\Delta(G)=3$;

2. (Burstein [26]) $A(G) \leq 5$ if $\Delta(G)=4$;

3. (Yadav, Varagani, Kothapalli, Venkaiah [94]) $A(G) \leq 8$ if $\Delta(G)=5$;

4. (Varagani, Venkaiah, Yadav, Kothapalli [87]) $A(G) \leq 12$ if $\Delta(G)=6$.

By applying Theorem 2.3.8, the following (unsatisfactory) bounds are obtained on $\chi_{\Sigma}^{v}(G)$ :

Corollary 2.3.10. If $G$ is a graph with maximum degree $\Delta(G)$, then

1. $\chi_{\Sigma}^{v}(G) \leq 3 \times 5 \times 7 \times 11 \times 13 \times 17=255255$ if $\Delta(G)=3$;

2. $\chi_{\Sigma}^{v}(G) \leq 3 \times 5 \times 7 \times \cdots \times 31=100280245065$ if $\Delta(G)=4$;

3. $\chi_{\Sigma}^{v}(G) \leq 3 \times 5 \times 7 \times \cdots \times 157 \approx 1.769 \times 10^{61}$ if $\Delta(G)=5$; and

4. $\chi_{\Sigma}^{v}(G) \leq 3 \times 5 \times 7 \times \cdots \times 331 \approx 3.204 \times 10^{132}$ if $\Delta(G)=6$.

As with the previously considered variations on the 1-2-3 Conjecture, one may vary the operation used to obtain a vertex colouring from a vertex weighting. In [32], Chartrand, Okamoto, Salehi, and Zhang study vertex weightings which properly colour vertices by multisets. Aside from establishing the easy upper bound of $\chi(G)$ for $\chi_{m}^{v}(G)$, exact values of $\chi_{m}^{v}(G)$ are determined for bipartite graphs, complete multipartite graphs, and powers of cycles.

Colouring by sets are considered in [31] by Chartrand, Okamoto, Rasmussen, and Zhang, who show that $\chi_{s}^{v}(G) \leq \chi(G)$ and that $\chi_{s}^{v}(G)$ is bounded below by 
$\left\lceil\log _{2}(\chi(G)+1)\right\rceil$ and by $1+\left\lceil\log _{2} \omega(G)\right\rceil$. Particular values are determined for some graph classes, and the effects of vertex and edge deletions are considered. This parameter is closely related to a graph's locally identifying chromatic number, which is the fewest number of colours needed to properly colour $V(G)$ such that the set of colours in $N_{G}[u]$ differs from the set of colours in $N_{G}[v]$ for any adjacent vertices $u, v$ with $N_{G}[u] \neq N_{G}[v]$. This concept was introduced in [45] by Esperet et al., who show that there exist graphs which require $\Omega\left(\Delta(G)^{2}\right)$ colours. They ask whether or not $O\left(\Delta(G)^{2}\right)$ colours suffices for every admissible graph; this was answered in the affirmative by Foucould et al. in [46].

\subsection{Variation III: List weightings}

A popular variation of many colouring problems is to colour elements from independently assigned lists rather than from one universal colour set. Research on listcolouring problems often provides great insight into classical colouring problems. For instance, Thomassen's proof that $\operatorname{ch}(G) \leq 5$ for any planar graph $G$ [85] is arguably the most elegant proof of the classical result that every planar graph $G$ has $\chi(G) \leq 5$.

Similarly, we hope to gain insight on the 1-2-3 Conjecture by considering colourings that come from list-weightings. Recall that, in a list-weighting of a graph $G$, each edge or vertex receives a weight from its own independently assigned list of real numbers; a colouring is then derived using some binary operation. Let $\operatorname{ch}_{\Sigma}^{e}(G)$ denote the smallest value of $k$ for which assigning a list of size $k$ of permissible weights, called a $k$-list assignment, to each edge of a graph permits an edge $k$-list-weighting which is a proper vertex colouring by sums; each of the parameters given thus far generalizes similarly. 
Note that we refer to lists of "permissible" weights begin assigned to edges, vertices, or both in the preceding paragraph. For most colouring operations, these lists may be chosen freely from $\mathbb{R}$. However, if one were to colour vertices by products from an edge weighting, it is clear that one would never choose an edge weight to be 0 as this would colour each of its ends 0 . Hence, we exclude the edge weight 0 when colouring by products from edge list-weightings (for total and vertex list-weightings, 0 is permissible for vertex lists).

\subsubsection{Edge list-weightings}

We will be primarily motivated by the following conjecture, a strengthening of the 1-2-3 Conjecture:

List 1-2-3 Conjecture (Bartnicki, Grytczuk, Niwcyk [15]). For every nice graph G, $\operatorname{ch}_{\Sigma}^{e}(G) \leq 3$

While one may be tempted to conjecture that $\operatorname{ch}_{\Sigma}^{e}(G)=\chi_{\Sigma}^{e}(G)$ for any graph $G$, we will see that this is not the case for graphs as simple as paths (Proposition 3.1.2).

The authors of the List 1-2-3 Conjecture have developed an approach to the problem which makes use of Alon's Combinatorial Nullstellensatz; this approach is used in [15] to show that $\operatorname{ch}_{\Sigma}^{e}(G) \leq 3$ if $G$ is complete, complete bipartite, or a tree.

One may easily extend the concept of vertex colouring edge weightings of graphs to the realm of digraphs. For an arc-weighting of a digraph $D$, the natural way to define a vertex colouring by sums is to define the colour of a vertex as the sum of the weights of its incident incoming arcs less the sum of the weights of its incident outgoing arcs. Such vertex colouring edge weightings are completely understood; a constructive method is used to prove the following result: 
Theorem 2.4.1 (Bartnicki, Grytczuk, Niwcyk [15]). For any digraph $D, \operatorname{ch}_{\Sigma}^{e}(D) \leq 2$.

\subsubsection{Total list-weightings}

Just as the 1-2-3 Conjecture has a natural list generalization, so too does the 1-2 Conjecture.

List 1-2 Conjecture (Przybyło, Wozniak [77]). For every graph $G, \operatorname{ch}_{\Sigma}^{t}(G) \leq 2$.

In [92], Wong and Zhu study $(k, l)$-total list-assignments, which are assignments of lists of size $k$ to the vertices of a graph and lists of size $l$ to the edges. If any $(k, l)$-total list-assignment of $G$ permits a total weighting which is a proper vertex colouring by sums, then $G$ is $(k, l)$-weight choosable. Obviously, if a graph $G$ is $(k, l)$-weight choosable, then $\operatorname{ch}_{\Sigma}^{t}(G) \leq \max \{k, l\}$. Wong and Zhu make the following two conjectures which, if true, would be stronger than the List 1-2-3 and List 1-2 Conjectures:

Conjecture 2.4.2 (Wong, Zhu [92]). Every graph is $(2,2)$-weight choosable. Every nice graph is $(1,3)$-weight choosable.

The most current work on $(k, l)$-weight choosability focuses on graph classes which are $(k, l)$-weight choosable for small values of $k$ and $l$ (see [15], [77], [93], [92]). Table 1 on page 29 summarizes these results, most of which are proven using the Combinatorial Nullstellensatz approach given in [15].

Horn̆ák and Woźniak [56] consider the list variation of $\chi_{s}^{e}(G)$ (or $\operatorname{gndi}(G)$ ). They denote this parameter $\operatorname{gln} \operatorname{di}(G)$, while we denote it $\operatorname{ch}_{s}^{e}(G)$. They show that $\operatorname{ch}_{s}^{e}(G)=\chi_{s}^{e}(G)=\left\lceil\log _{2} \chi(G)\right\rceil+1$ in the case where $G$ is a path or cycle, and that $\operatorname{ch}_{s}^{e}(T) \leq 3$ for any tree $T$. If the edge weighting is required to give a proper edge 


\begin{tabular}{|c|c|}
\hline Type of graph & $(k, l)$-weight choosability \\
\hline$K_{2}$ & $(2,1)$ \\
$K_{n}, n \geq 3$ & $(1,3),(2,2)$ \\
$K_{n, m}, n \geq 2$ & $(1,2)$ \\
$K_{m, n, 1, \ldots, 1}$ & $(2,2)$ \\
trees & $(1,3),(2,2)$ \\
unicyclic graphs & $(2,2)$ \\
generalized theta graphs & $(1,3),(2,2)$ \\
wheels & $(2,2)$ \\
\hline
\end{tabular}

Table 1: $(k, l)$-weight choosability of classes of graphs

colouring as well, they denote the corresponding parameter $\operatorname{lndi}(G)$ in correspondence with the parameter $\operatorname{ndi}(G)$ for the non-list version of the problem. They show that $\operatorname{lndi}(G)=\operatorname{ndi}(G)$ if $G$ is a cycle or a tree and conjecture that $\operatorname{lndi}(G)=\operatorname{ndi}(G)$ for every graph $G$, a conjecture reminiscent of the List Colouring Conjecture (see [58] for a history of the problem).

List Colouring Conjecture. For any graph $G, \operatorname{ch}^{\prime}(G)=\chi^{\prime}(G)$.

Note that a vertex list-colouring of a graph is in fact a special case of a total list-weighting which gives a vertex colouring by sums; if each edge is given the list $\{0\}$ and each vertex is given an arbitrary list $L_{v}$, then a total list-weighting which is a vertex colouring by sums is precisely a vertex list-colouring from $\left\{L_{v}: v \in V(G)\right\}$.

There is an easy to prove relationship between the weighting parameters $\operatorname{ch}_{\Sigma}^{t}(G)$, $\operatorname{ch}_{\Pi}^{t}(G)$, and $\operatorname{ch}_{m}^{t}(G)$ and the choosability number of $G, \operatorname{ch}(G)$ (part 1 is also shown by Wong and Zhu in [92]). 
Proposition 2.4.3. For every nontrivial connected graph $G$,

1. $\operatorname{ch}_{\Sigma}^{t}(G) \leq \operatorname{ch}(G)$

2. $\operatorname{ch}_{\Pi}^{t}(G) \leq \operatorname{ch}(G)$, and

3. $\operatorname{ch}_{m}^{t}(G) \leq \operatorname{ch}(G)$.

Proof. In each case, we prove a stronger statement about total $(\operatorname{ch}(G), 1)$-listweightings.

1. Consider a total $(\operatorname{ch}(G), 1)$-list-weighting, $\phi$, from vertex weight lists $\left\{L_{v} \mid v \in V(G)\right\}$ and edge weights $\{w(e) \mid e \in E(G)\}$. For each vertex $v \in V(G)$, let $L_{v}^{+}=\left\{w+\sum_{e \ni v} w(e) \mid w \in L_{v}\right\}$. Since $\left|L_{v}^{+}\right|=\operatorname{ch}(G)$, there is a proper colouring from the lists $\left\{L_{v}^{+} \mid v \in V(G)\right\}$ and hence $\phi$ gives a proper vertex colouring by sums.

2. Let $\dot{\psi}$ be a total $(\operatorname{ch}(G), 1)$-list-weighting from vertex weight lists $\left\{L_{v} \mid v \in V(G)\right\}$ and edge weights $\{w(e) \mid e \in E(G)\}$. For each vertex $v \in V(G)$, let $L_{v}^{+}=\left\{w \prod_{e \ni v} w(e) \mid w \in L_{v}\right\}$. Since $\left|L_{v}^{+}\right|=\operatorname{ch}(G)$, there is a proper colouring from the lists $\left\{L_{v}^{+} \mid v \in V(G)\right\}$ and hence $\psi$ gives a proper vertex colouring by products.

3. Since $\operatorname{ch}_{m}^{t}(G) \leq \operatorname{ch}_{\Sigma}^{t}(G)$ and $\operatorname{ch}_{m}^{t}(G) \leq \operatorname{ch}_{\Pi}^{t}(G)$ for every graph $G$, the result follows.

Part 1 of Proposition 2.4.3 implies the following corollary:

Corollary 2.4.4. A graph $G$ has a total $(k, 1)$-list-weighting which is a proper vertex colouring by sums if and only if $k \geq \operatorname{ch}(G)$. 
Proof. If $k \geq \operatorname{ch}(G)$, then $G$ has a total $(k, 1)$-list-weighting by part 1 of Proposition 2.4.3. If $\phi$ is a total list-weighting where each edge receives weight 0 , then a vertex colouring by sums from $\phi$ corresponds precisely to a vertex list-colouring of $G$. If $k<\operatorname{ch}(G)$, then this colouring cannot be proper.

Having established these bounds, it follows that the following graphs which have low choosability number also have small values of $\operatorname{ch}_{\Sigma}^{t}(G), \operatorname{ch}_{\Pi}^{t}(G)$ and $\operatorname{ch}_{m}^{t}(G)$ :

Proposition 2.4.5. Let $n$ be a positive integer.

1. If $G$ is a graph whose core is $K_{1}, C_{2 n+2}$, or $\theta_{2,2,2 n}$, then $\max \left\{\operatorname{ch}_{\Sigma}^{t}(G), \operatorname{ch}_{\Pi}^{t}(G), \operatorname{ch}_{m}^{t}(G)\right\} \leq 2$.

2. If all cycles of $G$ have length divisible by an integer $k \geq 3$, then $\max \left\{\operatorname{ch}_{\Sigma}^{t}(G), \operatorname{ch}_{\mathrm{II}}^{t}(G), \operatorname{ch}_{m}^{t}(G)\right\} \leq 3$.

3. If $G$ is planar, then $\max \left\{\operatorname{ch}_{\Sigma}^{t}(G), \operatorname{ch}_{\Pi}^{t}(G), \operatorname{ch}_{m}^{t}(G)\right\} \leq 5$.

4. If $G$ is bipartite and planar then $\max \left\{\operatorname{ch}_{\Sigma}^{t}(G), \operatorname{ch}_{\Pi}^{t}(G), \operatorname{ch}_{m}^{t}(G)\right\} \leq 3$.

Proof. 1. If $G$ has $K_{1}, C_{2 n+2}$, or $\theta_{2,2,2 n}$ as its core, then $\operatorname{ch}(G) \leq 2$ (Erdös, Rubin, Taylor $[44])$.

2. If every cycle in $G$ has length $0(\bmod k)$, then $\operatorname{ch}(G) \leq 3$ (Akbari, Ghanbari, Jahanbekam, Jamaali [6]).

3. If $G$ is planar, then $\operatorname{ch}(G) \leq 5$ (Thomassen [85]).

4. If $G$ is bipartite and planar, then $\operatorname{ch}(G) \leq 3$ (Alon, Tarsi [7]).

Furthermore, every graph $G$ has the following two upper bounds on $\operatorname{ch}_{\Sigma}^{t}(G)$, $\operatorname{ch}_{\Pi}^{t}(G)$, and $\operatorname{ch}_{m}^{t}(G)$ : 
Proposition 2.4.6. If $G$ is a graph on $n$ vertices, then

1. $\max \left\{\operatorname{ch}_{\Sigma}^{t}(G), \operatorname{ch}_{\Pi}^{t}(G), \operatorname{ch}_{m}^{t}(G)\right\} \leq \Delta(G)+1$, and

2. $\max \left\{\operatorname{ch}_{\Sigma}^{t}(G), \operatorname{ch}_{\Pi}^{t}(G), \operatorname{ch}_{m}^{t}(G)\right\} \leq \chi(G) \log n$.

Proof. The first upper bound follows from $\operatorname{ch}(G) \leq \Delta(G)+1$ (Erdös, Rubin, Taylor [44]; Vizing [89]). The second upper bound follows from $\operatorname{ch}(G) \leq \chi(G) \log n$ (Eaton [42]).

We conclude with a consideration of colouring vertices by products from list weightings. By excluding 0 from our ground set of potential edge weights, it is possible that $\operatorname{ch}_{\mathrm{II}}^{e}(G) \leq 3$ (were we not to exclude 0 , the lists $\{-1,0,1\}$ would not permit a vertex colouring for a nice non-bipartite graph). The following proposition shows that, while we hope that $\operatorname{ch}_{\Sigma}^{t}(G) \leq 2$ for any graph $G$, a similar bound is not possible for all graphs when colouring by products.

Proposition 2.4.7. If $G$ is a graph with $\chi(G) \geq 3$, then both $\operatorname{ch}_{\Pi}^{e}(G)$ and $\operatorname{ch}_{\Pi}^{t}(G)$ are at least 3 .

Proof. Suppose $\chi(G) \geq 3$ and $\operatorname{ch}_{\Pi}^{t}(G) \leq 2$. For each $e \in E(G)$ and each $v \in V(G)$, let $L_{e}=L_{v}=\{-1,1\}$. Let $c(v)$ denote the product of weights associated with $v$ from either an edge list-weighting or total list-weighting from these lists. In either case, we have $c(v) \in\{1,-1\}$, and so $c$ cannot properly colour $V(G)$.

The following proposition shows that $\operatorname{ch}_{\Pi}^{e}(G)$ and $\operatorname{ch}_{\Pi}^{t}(G)$ can be bounded by functions of $\operatorname{ch}_{\Sigma}^{e}(G)$ and $\operatorname{ch}_{\Sigma}^{t}(G)$, respectively. To avoid confusion between the number $e$ and an edge $e \in E(G)$, the function $e^{x}$ is denoted $\exp (x)$ in the proof below. 
Proposition 2.4.8. If $G$ is a nice graph, then $\operatorname{ch}_{\mathrm{II}}^{e}(G) \leq 2 \mathrm{ch}_{\Sigma}^{e}(G)$. For any graph $G$, $\operatorname{ch}_{\Pi}^{t}(G) \leq 2 \operatorname{ch}_{\Sigma}^{t}(G)+1$.

Proof. Let $L$ be a list of distinct numbers from $\mathbb{R} \backslash\{0\}$, and denote by $\log L$ the list of distinct numbers $\log L=\{\log |x|: x \in L\}$. The set $\left\{ \pm e^{y}: y \in \log L\right\}$ contains $L$ as a subset, and so $2|\log L| \geq|L|$.

Let $G$ be a nice graph with $\operatorname{ch}_{\Sigma}^{e}(G)=k$. Assign to each edge $e \in E(G)$ a list $L_{e}$ of $(2 k+1)$ real numbers. For each $e \in E(G)$, consider $\log L_{e}$, which has size at least $k$. There exists a choice of weights $w(e) \in \log L_{e}$ for each $e \in E(G)$ such that $\sum_{e \ni u} w(e) \neq \sum_{e \ni v} w(e)$ for each $u v \in E(G)$. This implies that $\exp \left(\sum_{e \ni u} w(e)\right) \neq \exp \left(\sum_{e \ni v} w(e)\right)$, and hence $\prod_{e \ni u} \exp (w(e)) \neq \prod_{e \ni v} \exp (w(e))$. Both products are positive, and so certainly $\prod_{e \ni u} \exp (w(e)) \neq \pm \prod_{e \ni v} \exp (w(e))$. Since either $\exp (w(e)) \in L_{e}$ or $-\exp (w(e)) \in L_{e}$ for each $e \in E(G)$, the weighting function $w^{\prime}(e)=\exp (w(e))$ colours $V(G)$ by products from the lists $\left\{L_{e}: e \in E(G)\right\}$.

By excluding the possible vertex weight 0 before taking the logarithms of potential weights, the result on total list-weightings follows by a similar argument.

\subsubsection{Vertex list-weightings}

Little has been published on the topic of colourings from list vertex weightings. Denote by $\operatorname{ch}_{\Sigma}^{v}(G)$ the smallest $k$ such that $G$ has an additive colouring from any assignment of lists of size $k$ to the vertices of $G$, and call $\operatorname{ch}_{\Sigma}^{v}(G)$ the additive choosability number of $G$. Such list-weightings were considered by Czerwiński et al. [36], who show that if $T$ is a tree, then $\operatorname{ch}_{\Sigma}^{v}(T) \leq 2$, and if $G$ is a bipartite planar graph, then $\operatorname{ch}_{\Sigma}^{v}(G) \leq 3$. 


\subsection{Variation IV: Edge colourings}

\subsubsection{Edge colourings}

Each section so far has dealt with the problem of properly colouring the vertices of a graph given an edge weighting, vertex weighting, or total weighting. We now define how one might similarly define edge colourings and total colourings from a weighting.

Given an edge $k$-weighting, we colour an edge $e \in E(G)$ with the sum, product, multiset, or set of weights from edges adjacent to $e$. If the resulting edge colouring is proper, then the $k$-edge weighting of $G$ is a proper edge colouring by sums, products, multisets or sets, respectively. The smallest $k$ for which such colourings exist for a graph $G$ are denoted $\chi_{\Sigma}^{\prime e}(G), \chi_{\Pi}^{\prime e}(G), \chi_{m}^{\prime e}(G)$ and $\chi_{s}^{\prime e}(G)$, respectively.

These parameters do not appear in the present literature, but a few bounds are easily obtained by noting that each is a particular case of its vertex-weighting vertexcolouring analog, e.g., $\chi_{\Sigma}^{\prime e}(G)=\chi_{\Sigma}^{v}(L(G)), \operatorname{ch}_{\Sigma}^{\prime e}(G)=\operatorname{ch}_{\Sigma}^{v}(L(G))$, etc. In the particular case of $\chi_{\Sigma}^{\prime e}(G)$, we have a few easy to prove upper bounds. Theorem 2.3.8 bounds $\chi_{\Sigma}^{\prime e}(G)$ in terms of the acyclic chromatic number of $L(G)$. The case of proper colouring by multisets also has a bound which follows from previous work.

Proposition 2.5.1. For any graph $G, \chi_{m}^{\prime e}(G) \leq \chi^{\prime}(G)$.

Proof. By Proposition 2.3.6, $\chi_{m}^{v}(L(G)) \leq \chi(L(G))$. Since $\chi_{m}^{\prime e}(G)=\chi_{m}^{v}(L(G))$, the result follows.

One may also colour edges from a vertex or total weighting, or by list weightings of each type; such scenarios have yet to be studied.

A total $k$-weighting of a graph $G, w: V(G) \cup E(G) \rightarrow[k]$ is a proper edge colouring by sums if the edge colouring function $c(u v)=w(u)+w(v)+w(u v)$ 
is proper. The smallest value of $k$ such that there exists a total $k$-weighting which is a proper edge colouring by sums is denoted $\chi_{\Sigma}^{\prime t}(G)$. While one may easily define similar parameters for proper colourings by products, multisets, or sets, only $\chi_{\Sigma}^{\prime t}(G)$ has been explicitly studied in published literature.

Proposition 2.5.2 (Brandt, Budajová, Rautenbach, Stiebitz [22]). If a graph G has maximum degree $\Delta(G)=\Delta$, then $\chi_{\Sigma}^{\prime t}(G) \leq \frac{\Delta}{2}+O(\sqrt{\Delta \log \Delta})$.

Brandt et al. also establish bounds for trees and complete graphs, and give necessary and sufficient conditions for a cubic graph $G$ to have $\chi_{\Sigma}^{\prime t}(G)=2$.

The topic of weightings and list-weightings which are proper total colourings via some binary operation remains unstudied.

\subsection{Variation V: Distinguishing colourings}

As mentioned, one motivation for the 1-2-3 Conjecture comes from graph irregularity strength. Recall that the irregularity strength of a graph $G$ is the smallest positive integer $k$ for which there exists an edge weighting $w: E(G) \rightarrow[k]$ such that every vertex of $G$ has a distinct sum of incident edge weights; that is, $\sum_{e \ni u} w(e) \neq \sum_{e \ni v} w(e)$ for all $u, v \in V(G)$. The original notation for the irregularity strength of a graph $G$, given by Chartrand et al. [29], is $\mathrm{s}(G)$; to maintain consistency with the notation developed so far, we will denote it $s_{\Sigma}^{e}(G)$. There is a natural "global" variation of each of the "local" parameters defined so far. For the local parameters $\chi_{x}^{y}(G), \chi_{x}^{\prime y}(G)$, and $\chi_{x}^{\prime \prime}(G)$, where $x \in\left\{\sum, \Pi, m, s\right\}$ and $y \in\{e, v, t\}$, let $\mathrm{s}_{x}^{y}(G), \mathrm{s}_{x}^{\prime y}(G)$, and $\mathrm{s}_{x}^{\prime \prime y}(G)$ denote their respective "global" counterparts. Similarly, let $\operatorname{ls}_{x}^{y}(G), \operatorname{ls}_{x}^{\prime y}(G)$, and $\operatorname{ls}_{x}{ }_{x}^{y}(G)$ denote the global versions of $\operatorname{ch}_{x}^{y}(G), \operatorname{ch}_{x}^{\prime y}(G)$, and $\operatorname{ch}_{x}^{\prime \prime}(G)$, respectively. 
Irregularity strength and its variations have generated a significant amount of interest since the topic's inception in 1986. Due to the breadth of work done in the field, and since it is not a primary focus of this thesis, we will highlight some of the more significant results in the field in lieu of providing a complete survey (see [48], [67], [91] for more results).

\subsubsection{Distinguishing elements by edge-weightings}

A major conjecture on the topic of irregularity strength was one of Aigner and Triesch [4], who asked if $s_{\Sigma}^{e}(G) \leq|V(G)|-1$ for every nice graph. This conjecture was answered in the affirmative by Nierhoff [73]. The following is the best known irregularity strength bound:

Theorem 2.6.1 (Kalkowski, Karoński, Pfender [63]). For any nice graph $G$, $\mathrm{s}_{\Sigma}^{e}(G) \leq\lceil 6 n / \delta(G)\rceil$

Recently, Anholcer and Cichacz proved a group analog of Aigner and Triesch's conjecture. They define the group irregularity strength of a graph $G, s_{g}(G)$, to be the smallest value of $s$ such that, for any abelian group $(\Gamma,+)$ of order $s$, there exists an edge weighting of $G$ from $\Gamma$ such that distinct vertices receive distinct sums of edge weights.

Theorem 2.6.2 (Anholcer, Cichacz [11]). For any connected graph of order $n \geq 3$, $n \leq s_{g}(G) \leq n+2$.

As with their result on group sum chromatic numbers (Theorem 2.2.12), Anholcer and Cichacz characterize which graphs have group irregularity strength $n, n+1$, and $n+2$. 
Note that irregular weightings are closely related to antimagic labelings. A graph $G$ with $m$ edges is called antimagic if there exists a bijection between $E(G)$ and $\{1, \ldots, m\}$ such that every vertex has a distinct sum of edge weights from its adjacent edges. Such a bijection is called an antimagic labeling. Antimagic labelings and their variations are widely studied; a thorough survey may be found in Chapter 6 of [48].

Aigner et al. [5] introduce a version of irregularity strength where each vertex is assigned the multiset of edge weights from incident edges rather than the sum; the corresponding parameter is $\mathrm{s}_{m}^{e}(G)$ in our notation and is called the multiset irregularity strength of $G$. The following result is proven using probabilistic methods:

Theorem 2.6.3 (Aigner, Triesch, Tuza [5]). If $G$ is a d-regular graph, then $\mathrm{s}_{m}^{e}(G) \in \Theta\left(n^{1 / d}\right)$.

Anholcer [9] studied the product irregularity strength of a graph, or $\mathrm{s}_{\Pi}^{e}(G)$. He establishes bounds on $\mathrm{s}_{\Pi}^{e}(G)$ when $G$ is a cycle, path or grid. Pikhurko [74] has shown that if a graph is sufficiently large, then $\mathrm{s}_{\Pi}^{e}(G) \leq|E(G)|$ (in antimagic labelling terminology, he shows that sufficiently large graphs are product antimagic).

A vertex-distinguishing edge-colouring of a graph $G$ is a colouring of the edges such that for any two vertices $u$ and $v$, the set of colours assigned to the set of edges incident to $u$ differs from the set of colours assigned to the set of edges incident to $v$. Harary and Plantholt [54] introduced the study of vertex-distinguishing proper edge-colourings in 1985; they use $\chi_{0}(G)$ to denote the smallest value of $k$ such that a graph $G$ has such an edge-colouring, and they call the parameter the pointdistinguishing chromatic index of $G$. Note that $\chi_{0}(G)$ is a global version of $\chi_{s}^{e}(G)$ with the added constraint that the edge weighting is a proper edge-colouring, 
and hence is an upper bound on $\mathrm{s}_{s}^{e}(G)$.

Theorem 2.6.4 (Burris, Schelp [25]). If $n_{i}$ denotes the number of vertices of degree $i$ in a graph $G$, then for any nice graph $G, \chi_{0}(G) \leq C \max \left\{n_{i}^{1 / i}: 1 \leq i \leq \Delta(G)\right\}$, where $C$ is a constant relying only on $\Delta(G)$.

Burris and Schelp also make the following two conjectures:

Conjecture 2.6.5 (Burris, Schelp [25]). 1. Let $G$ be a simple graph with no more than one isolated vertex and no connected component isomorphic to $K_{2}$. If $k$ is the minimum integer such that $\left(\begin{array}{l}k \\ d\end{array}\right) \geq n_{d}$ for all $\delta(G) \leq d \leq \Delta(G)$, then $\chi_{0}(G) \in\{k, k+1\}$

2. If $G$ is a simple graph with no more than one isolated vertex and no connected component isomorphic to $K_{2}$, then $\chi_{0}(G) \leq|V(G)|+1$.

The second conjecture was answered in the affirmative by Bazgan et al. [17].

\subsubsection{Distinguishing elements by total- and vertex- weightings}

Bača, Jendrol', Miller, and Ryan introduced the total edge irregularity strength of a graph $G$ in [16], which is defined to be the smallest $k$ for which there is a total $k$-weighting $w$ such that $w(u)+w(v)+w(u v) \neq w(x)+w(y)+w(x y)$ for any distinct $u v, x y \in E(G)$. In our notation, this parameter is $\mathrm{s}_{\Sigma}^{\prime t}(G)$. They show that if $G$ has $m$ edges, then $\left\lceil\frac{m+2}{3}\right\rceil \leq \mathrm{s}_{\Sigma}^{t}(G) \leq m$. They also make the following conjecture:

Conjecture 2.6.6 (Bača, Jendrol', Miller, Ryan [16]). If $G \nsubseteq K_{5}$, then

$$
\mathrm{s}_{\Sigma}^{\prime t}(G)=\max \left\{\left\lceil\frac{\Delta(G)+1}{2}\right\rceil,\left\lceil\frac{|E(G)|+2}{3}\right\rceil\right\}
$$


This conjecture was recently confirmed in [23] for graphs with $|E(G)|>111000 \Delta(G)$ and for a random graph from $\mathcal{G}(n, p)$ for any $p=p(n)$. It is also shown in $[23]$ that $\mathrm{s}_{\Sigma}^{\prime t}(G) \leq\left\lceil\frac{|E(G)|}{2}\right\rceil$ for any graph $G$ which is not a star. The conjecture is also verified in [24] for any graph $G$ having $|E(G)| \leq \frac{3}{2}|V(G)|-1$ and $\Delta(G) \leq\left\lceil\frac{|E(G)|+2}{3}\right\rceil$.

Bača et al. [16] also introduce the total vertex irregularity strength of a graph $G$, denoted $\mathrm{s}_{\Sigma}^{t}(G)$, which is the smallest $k$ such that $G$ has a total $k$-weighting $w$ such that $w(u)+\sum_{e \ni u} w(e) \neq w(v)+\sum_{e \ni v} w(e)$ for any distinct $u, v \in V(G)$. The best known bound on $\mathrm{s}_{\Sigma}^{t}(G)$ was given by Anholcer, Kalkowski, and Przybyło [10], who showed that $\mathrm{s}_{\Sigma}^{t}(G) \leq 3\lceil n / \delta\rceil+1$ for a graph on $n$ vertices with minimum degree $\delta(G)=\delta$. For a survey of results on total edge irregularity strength and total vertex irregularity strength, see Section 7.17 in [48].

Chartrand, Lesniak, VanderJagt, and Zhang [30] have very recently considered vertex weightings for which the multiset of colours of the vertices in $N(u)$ differs from the multiset of colours of the vertices of $N(v)$ for any distinct vertices $u$ and $v$. The smallest $k$ such that a graph $G$ has such a vertex $k$-weighting is denoted $\mathrm{s}_{m}^{v}(G)$. Such weightings are also known as recognizable colourings and $\mathrm{s}_{m}^{v}(G)$ is the recognition number of $G$. Recognition numbers are determined in [30] for a number of classes of graphs.

An edge-distinguishing vertex weighting of a graph $G$ is a weighting $w: V(G) \rightarrow[k]$ such that $\{w(u), w(v)\} \neq\{w(x), w(y)\}$ for any two distinct edges $u v, x y \in E(G)$. Such weightings were introduced by Frank, Harary and Plantholt [47], where they were called line-distinguishing vertex colourings. The smallest value of $k$ for which a graph $G$ has an edge-distinguishing vertex-weighting is 
called the line-distinguishing chromatic number, and is denoted $\mathrm{s}_{s}^{\prime v}(G$ ) (note that $\{w(u), w(v)\} \neq\{w(x), w(y)\}$ as sets if and only if they are distinct as multisets, and hence we could also use $\mathrm{s}_{m}^{v}(G)$ ). Harary and Plantholt [53] conjectured that $\mathrm{s}_{m}^{\prime v}(G) \geq \chi^{\prime}(G)$ for every graph $G$, which was proven by Salvi [79].

\subsection{Summary}

\section{Significant conjectures}

1-2-3 Conjecture (Karoński, Luczak, Thomason [64]). If $G$ is nice, then $\chi_{\Sigma}^{e}(G) \leq 3$.

1-2 Conjecture (Przybyło, Wozniak [76]). For every graph $G, \chi_{\Sigma}^{t}(G) \leq 2$.

List 1-2-3 Conjecture (Bartnicki, Grytczuk, Niwcyk [15]). For every nice graph G, $\operatorname{ch}_{\Sigma}^{e}(G) \leq 3$

List 1-2 Conjecture (Przybyło, Wozniak [77]). For every graph $G, \operatorname{ch}_{\Sigma}^{t}(G) \leq 2$.

Wong and Zhu [92] further conjecture that every graph is $(2,2)$-weight choosable and every nice graph is $(1,3)$-weight choosable.

Additive Colouring Conjecture (Czerwiński, Grytczuk, Żelazny [36]). For every $\operatorname{graph} G, \chi_{\Sigma}^{v}(G) \leq \chi(G)$.

\section{Thesis overview}

While it is known that, asymptotically almost surely, a random graph $G$ has $\chi_{\Sigma}^{e}(G) \leq 2$, no characterization is known for graphs with $\chi_{\Sigma}^{e}(G) \leq 2$. In Chapter 3 , we will establish a variety of classes of graphs for which this bound holds. In many 
cases the results given provide generalizations of results given in [28], in that they hold for weighting sets of size two other than $\{1,2\}$. We will also prove that there is a set of minimal graphs, with respect to subgraph containment, for which $\chi_{\Sigma}^{e}(G)>2$.

Chapter 4 is concerned with the List 1-2-3 Conjecture, List 1-2 Conjecture, and the list variation of the Additive Colouring Conjecture. While some classes of graphs have known values of $\operatorname{ch}_{\Sigma}^{e}(G)$, no bound for a general graph exists in the current literature on the subject. We will show how the Combinatorial Nullstellensatz can be used to prove that $\operatorname{ch}_{\Sigma}^{e}(G) \leq 2 \Delta(G)+1$. Using the same technique, we will show that $\operatorname{ch}_{\Sigma}^{t}(G) \leq\left\lceil\frac{2}{3} \Delta(G)\right\rceil+1$, an improvement on the "trivial" upper bound of $\operatorname{ch}(G)$. The results presented are, in fact, bounds on the aforementioned $(k, l)$-weight choosability introduced by Wong and Zhu. The bound on $\operatorname{ch}_{\Sigma}^{e}(G)$ also implies an upper bound on the list size necessary to be able to properly colour vertices by products via an edge list-weighting. Finally, we establish a general bound on the additive choosability number of a graph $G, \operatorname{ch}_{\Sigma}^{v}(G)$, as well as improved upper bounds for particular graph classes. These results are a significant improvement on the bounds on $\chi_{\Sigma}^{v}(G)$ found in [36], presented earlier in Theorem 2.3.8 and Corollary 2.3.10.

The Combinatorial Nullstellensatz method presented in Chapter 4 is used in Chapter 5 to show that the natural digraph extension of the 1-2-3 Conjecture can be completely solved, answering a question posed in [15].

Just as there is a natural relaxation of the 1-2-3 Conjecture to a multiset colouring problem, so too can the multiset version be relaxed to sequences. Weightings which colour vertices by sequences are introduced in Chapter 6 ; this is the first study of this weighting problem. By imposing a global ordering on the edge set of a graph, a natural order is induced on the edges adjacent to a vertex. In the case where we are 
free to choose the global ordering as we like, will we show that any nice graph has an edge 2-list-weighting which properly colours the vertices of the graph by sequences. If we are not free to choose the ordering, then certainly four edge weights are sufficient to achieve a proper vertex colouring by sequences, since Theorem 2.2.3 states that four edge weights are sufficient for multisets. The 1-2-3 Conjecture would suggest that three edge weights should suffice. We consider the list-weighting version of the problem, with the primary tool being the Lovász Local Lemma. We will show that graphs which have sufficiently large minimum degree in terms of the maximum degree can be so weighted using edge weight lists of size 3 . These results are generalized to total weightings, and both sets of results are generalizable to multigraphs. A number of the results are further extended to a sequence relaxation of graph irregularity strength.

Finally, in Chapter 7, we introduce an edge weighting problem which can be motivated by the 1-2-3 Conjecture, fractional colourings, and injective colourings. In such an edge weighting, one colours vertices with multisets in the usual way, but with the requirement that adjacent vertices receive multisets of edge weights which are as different as possible; this differs from edge-weightings which properly colour vertices by multisets, which only require distinct multisets. Upper bounds are proven for the fewest number of edge weights needed for such a vertex colouring; these bounds are in terms of the injective chromatic number of line graphs and the chromatic number of powers of line graphs. A lower bound is given as a function of a graph's clique number. Other results are proven by using graph homomorphisms, and exact values for the fewest number of edge weights required are determined for some classes of graphs. 


\section{Chapter 3}

\section{Edge 2-Weightings Which Colour Vertices by Sums}

An earlier version of this chapter appears in [66], as a paper co-authored with Khatirinejad, Naserasr, Newman and Stevens.

\subsection{Preliminaries}

Recall the following two primary motivating conjectures on vertex colourings from edge weightings:

1-2-3 Conjecture. For every nice graph $G, \chi_{\Sigma}^{e}(G) \leq 3$.

List 1-2-3 Conjecture. For every nice graph $G, \operatorname{ch}_{\Sigma}^{e}(G) \leq 3$.

While the List 1-2-3 Conjecture is a natural generalization of the 1-2-3 Conjecture, there is a middle ground, of sorts, where every edge receives a weight from the same arbitrary set of size $k$. If a set of real numbers $S$ exists such that $G$ has an $S$-edge weighing which is a proper vertex colouring by sums, then $G$ is $S$-weight colourable. If $G$ is $S$-weight colourable for every set $S$ of size $k$, then $G$ is $k$-weight colourable. 
The problem of determining conditions under which a graph $G$ has $\chi_{\Sigma}^{e}(G) \leq 2$ was explored in [28] and [70]. In this chapter, we study the following, more general, problem:

Problem 3.1.1. For which graphs $G$ and for which $a, b \in \mathbb{R}$ is $G\{a, b\}$-weight colourable? In particular, which classes of graphs are 2-weight colourable?

Note that this is not a trivial generalization of determining which graphs $G$ have $\chi_{\Sigma}^{e}(G)=2$. For instance, although $\chi_{\Sigma}^{e}(T) \leq 2$ for every tree $T$ (see Corollary 3.2.7), paths of length $4 k+1$ are not $\{0, a\}$-weight colourable for any choice of $a$.

Proposition 3.1.2. Let $k$ be a positive integer and a a nonzero real number. If $P=v_{1} v_{2} \cdots v_{4 k+2}$ is a path, then $P$ is not $\{0, a\}$-weight colourable.

Proof. Suppose $w: E(P) \rightarrow\{0, a\}$ is a proper vertex colouring of $P$ by sums, and let $c(v)$ denote the colour of $v \in V$. If $w\left(v_{2} v_{3}\right)=0$, then $c\left(v_{1}\right)=w\left(v_{1} v_{2}\right)=w\left(v_{1} v_{2}\right)+w\left(v_{2} v_{3}\right)=c\left(v_{2}\right)$, a contradiction. Hence $w\left(v_{2} v_{3}\right)=a$ and, by a symmetric argument, $w\left(v_{4 k} v_{4 k+1}\right)=a$.

For any $1 \leq i \leq 4 k-1, w\left(v_{i} v_{i+1}\right) \neq w\left(v_{i+2} v_{i+3}\right)$, else $c\left(v_{i+1}\right)=c\left(v_{i+2}\right)$. Iteratively applying this inequality gives $w\left(v_{i} v_{i+1}\right)=w\left(v_{j} v_{j+1}\right)$ if $i \equiv j(\bmod 4)$ and $w\left(v_{i} v_{i+1}\right) \neq w\left(v_{j} v_{j+1}\right)$ if $i \equiv j-2(\bmod 4)$. Hence, $w\left(v_{2} v_{3}\right) \neq w\left(v_{4 k} v_{4 k+1}\right)$, a contradiction, so no such $w$ exists.

Whether or not a graph $G$ is $S$-weight colourable for a set $S \subset \mathbb{R}$ is not only dependent on the size of $S$ but also on the particular elements of $S$. However, if a graph $G$ is $S$-weight colourable, then there exists an $i_{0}=i_{0}(G, S)$ such that for all $i>i_{0}$ the graph is also $(S+i)$-weight colourable, where $S+i:=\{s+i: s \in S\}$. 
Proposition 3.1.3. Let $G$ be a nice graph which is $S$-weight colourable. If $M$ is the maximum element of $S$ and $m$ is the minimum element of $S$, then $G$ is $(S+i)$-weight colourable for every $i \geq(M+|m|) \Delta(G)$.

Proof. First note that, given an edge-weighting which colours vertices by sums, changing the sign of every edge weight gives a new edge weighting which also colours vertices by sums. This implies that we may assume, without loss of generality, that $M>0$.

Let $w: E(G) \rightarrow S$ be an edge weighting which is a proper vertex colouring by sums and let $c(v)$ be the induced colour of $v \in V(G)$. Let $i$ be a real number such that $i \geq(M+|m|) \Delta(G)$. Define $w_{0}(e)=w(e)+i$ for each $e \in E(G)$ and let $c_{0}(v)$ be the colour induced by $w_{0}$ for each $v \in V(G)$.

Consider an edge $u v \in E(G)$. Without loss of generality, assume that $d(u) \geq d(v)$. If $d(u)=d(v)$, then clearly $c(u) \neq c(v)$ implies that $c_{0}(u) \neq c_{0}(v)$. If $d(u)>d(v)$, then

$$
\begin{aligned}
c_{0}(u)-c_{0}(v) & =i d(u)+c(u)-i d(v)-c(v) \\
& =i(d(u)-d(v))+c(u)-c(v) \\
& \geq(M+|m|) \Delta(G)(d(u)-d(v))+m d(u)-M d(v) \\
& \geq(M+|m|) \Delta(G)(1)-|m| d(u)-M d(v) \\
& =M(\Delta(G)-d(v))+|m|(\Delta(G)-d(u)) \\
& \geq M(1)+|m|(0)>0 .
\end{aligned}
$$

Hence $c_{0}(u) \neq c_{0}(v)$ and so $c_{0}$ properly colours $V(G)$.

In Section 3.2, a wide range of basic graphs which admit $\{a, b\}$-weight colourings is established, as are some classes of graphs which do not admit $\{a, b\}$-weight colourings 
but which do admit an edge $\{a, b\}$-weighting which is almost a proper colouring by sums. These results provide building blocks for the results on the weight colourability of bipartite graphs presented in Section 3.3 and of other infinite classes of graphs in Sections 3.4 and 3.5 .

\subsection{Building blocks: Weight colourings of basic graphs}

From this point forward, $w$ denotes a weighting function on the edges of a graph $G$ and $c$ denotes the induced vertex colouring by sums. We begin with a few simple observations.

Proposition 3.2.1. Let $a, b, t$ be nonzero real numbers.

1. A graph $G$ is $\{a, b\}$-weight colourable if and only if $G$ is $\{a t, b t\}$-weight colourable.

2. If $G$ is $\{a, b\}$-weight colourable, then $G$ is $\{p, q\}$-weight colourable for any $p, q \in \mathbb{R} \backslash\{0\}$ which are linearly independent over $\mathbb{Q}$.

Proof. 1. This follows from the fact that, for two vertices $u v \in V(G), c(u) \neq c(v)$ if and only if $t \cdot c(u) \neq t \cdot c(v)$.

2. Let $w$ be an $\{a, b\}$-weight colouring of $G$, let $u v \in E(G)$, and suppose $c(u)=r a+s b$ and $c(v)=r^{\prime} a+s^{\prime} b$ for integers $r, r^{\prime} s, s^{\prime}$. Since $c(u) \neq c(v)$, either $r \neq r^{\prime}$ or $s \neq s^{\prime}$. Since $p$ and $q$ are linearly independent over $\mathbb{Q}$, $\left(r-r^{\prime}\right) p+\left(s-s^{\prime}\right) q \neq 0$ and so $r p+s q \neq r^{\prime} p+s^{\prime} q$. Hence, the edge weighting $w^{\prime}$ 
given by $w^{\prime}(e)=p$ if and only if $w(e)=a$ and $w^{\prime}(e)=q$ if and only if $w(e)=b$ is a $\{p, q\}$-weight colouring of $G$.

The following is deduced from Proposition 3.2.1, with the convention that 0 and 1 are relatively prime integers:

Corollary 3.2.2. A graph $G$ is 2 -weight colourable if and only if $G$ is $\{a, b\}$-weight colourable for every pair of relatively prime integers $a$ and $b$.

In other words, Proposition 3.2.1 allows one to prove a graph $G$ is $\{a, b\}$-weight colourable for many pairs of numbers by reducing to the case where $a$ and $b$ are relatively prime integers.

Proposition 3.2.3. If $G$ is $d$-regular and $\{a, b\}$-weight colourable for some $a, b \in \mathbb{R}$, then

1. $G$ is d-colourable, and

2. $G$ is 2-weight colourable.

Proof. 1. The colour $c$ of a vertex induced by an $\{a, b\}$-weighting must be a number of the form $t a+(d-t) b$ for some $0 \leq t \leq d$, and so an $\{a, b\}$-weighting gives $d+1$ different sums as colours. Clearly a vertex coloured $d a$ cannot be adjacent to a vertex coloured $d b$. The vertex colouring $c^{\prime}$ given by $c^{\prime}(v)=c(v)$ if $c(v) \notin\{d a, d b\}$ and $c^{\prime}(v)=d a$ otherwise gives a $d$-colouring of $G$.

2. In an edge $\{a, b\}$-weighting of a $d$-regular graph, vertex colours are in one-toone correspondence with the numbers of incident edges weighted $a$. Thus, the weight-colouring does not depend on the value of $a$ (or $b$ ), and so $\{a, b\}$ may be chosen arbitrarily. 
Corollary 3.2.4. If $\chi(G)=\Delta(G)+1$ (equivalently, by Brooks' theorem, $G$ is an odd cycle or a complete graph), then $G$ is not $\{a, b\}$-weight colourable for any $a, b \in \mathbb{R}$.

Unfortunately, $\chi(G)=\Delta(G)$ is neither sufficient nor necessary for a graph $G$ to be $\{1,2\}$-weight colourable. The graphs in Figure 1 each have $\chi(G)=\Delta(G)$. However, the graph on the left, known as the Penny graph, is $\{1,2\}$-weight colourable, while graph on the right in Figure 1, $K_{2} \square C_{3}$, is not (see Proposition 3.4.3). On the other hand, we will see that there exist bipartite graphs with arbitrarily high maximum degree which are $\{1,2\}$-weight colourable (e.g. trees; see Corollary 3.2.7) and which are not (e.g. generalized theta graphs of the form $\Theta_{\left(1,4 k_{2}+1, \ldots, 4 k_{d}+1\right)}$; see Theorem $3.2 .13)$
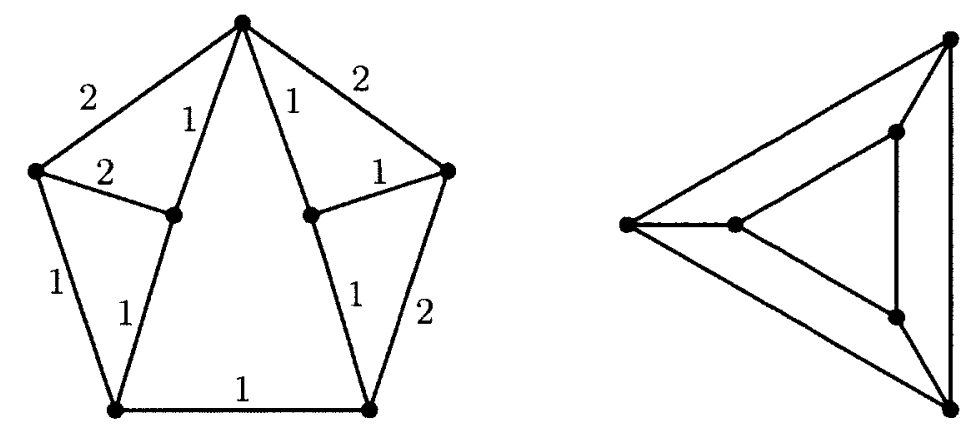

Figure 1: Graphs having $\chi(G)=\Delta(G)$ with different values of $\chi_{\Sigma}^{e}(G)$.

Even though complete graphs are not $S$-weight colourable for any set of size 2, they do have edge $S$-weightings that are very close to being an $S$-weight colouring. This specific weighting will be useful in constructing families of 2-weight colourable graphs and non-2-weight colourable graphs in Section 3.4.

Lemma 3.2.5. Given an integer $n \geq 2$ and $a, b \in \mathbb{R}, a \neq b$, there is an edge $\{a, b\}$ weighting of $K_{n}$ such that the colours of all the vertices are distinct except for two 
of them. Furthermore, in any such edge $\{a, b\}$-weighting, the degree sequence of the subgraph containing exactly the edges of weight a (as well as the subgraph induced by the edges of weight b) is either

$$
\left(1,2, \ldots,\left\lfloor\frac{n}{2}\right\rfloor-1,\left\lfloor\frac{n}{2}\right\rfloor,\left\lfloor\frac{n}{2}\right\rfloor,\left\lfloor\frac{n}{2}\right\rfloor+1, \ldots, n-2, n-1\right)
$$

or

$$
\left(0,1, \ldots,\left\lceil\frac{n}{2}\right\rceil-2,\left\lceil\frac{n}{2}\right\rceil-1,\left\lceil\frac{n}{2}\right\rceil-1,\left\lceil\frac{n}{2}\right\rceil, \ldots, n-3, n-2\right)
$$

Proof. Order the vertices $v_{1}, v_{2}, \ldots, v_{n}$. Call an edge incident to $v_{i}$ backwards (with respect to $v_{i}$ ) if it has the form $v_{i} v_{j}$ with $j<i$. If $i$ is even, weight all backwards edges incident to $v_{i}$ with $a$; otherwise weight the backwards edges incident to $v_{i}$ with b. We prove that this weighting colours $V\left(K_{n}\right)$ by sums as desired by induction on $n$. The cases of $n=2,3$ are trivial (note that $v_{1}$ and $v_{2}$ are the vertices with equal colours). Suppose the statement holds for $k<n$. Let $G \cong K_{n}$ be a graph on $V(G)=\left\{v_{1}, \ldots, v_{n}\right\}$ and $\{a, b\}$-weight colour the induced $K_{n-1}$ on $\left\{v_{1}, v_{2}, \ldots, v_{n-1}\right\}$ by induction. Note that $c\left(v_{n-1}\right)$ is $(n-2) a$ if $n$ is odd and $(n-2) b$ if $n$ is even, and that no other vertex is incident to a monochromatic set of edges. This implies that weighting the edges incident to $v_{n}$ (which are all backwards edges) according to the parity of $n$ gives the desired colouring, with $v_{1}$ and $v_{2}$ having equal colours.

We prove the second part of the lemma by induction on $n$. Suppose $w$ is an edge weighting of $K_{n}$ guaranteed by the first part of the statement of the lemma. Let $u$ and $v$ be the vertices such that $c(u)=c(v)$. It is easy to verify the claim for $n=2$ and $n=3$. If $c(x) \notin\{(n-1) a,(n-1) b\}$ for every vertex $x$, then $c(x)$ can only take $n-2$ values, a contradiction to the choice of $w$. If $c(u)=c(v) \in\{(n-1) a,(n-1) b\}$, 
then by removing $u$ and $v, w$ induces an $\{a, b\}$-weight colouring of $K_{n-2}$, a contradiction to Corollary 3.2.4. Thus, there exists a vertex $x \notin\{u, v\}$ such that $c(x) \in\{(n-1) a,(n-1) b\}$. Without loss of generality, say that $c(x)=(n-1) a$. This implies every vertex $v \in V\left(K_{n}\right) \backslash\{x\}$ is incident to at least one edge weighted b. Let $G=K_{n}-x$. Since $|V(G)|=n-1$, the degree sequence of the subgraph of $G$ containing exactly those edges weighted $a$ must be

$$
\left(0,1, \ldots,\left\lceil\frac{n-1}{2}\right\rceil-2,\left\lceil\frac{n-1}{2}\right\rceil-1,\left\lceil\frac{n-1}{2}\right\rceil-1,\left\lceil\frac{n-1}{2}\right\rceil, \ldots, n-4, n-3\right)
$$

by induction (note that the other possible degree sequence is not an option since it implies one vertex in $G$ is incident to only edges weighted $a$ ). Hence, the degree sequence of the subgraph of $K_{n}$ with edges weighted $a$ is

$$
\left(1,2, \ldots,\left\lceil\frac{n-1}{.2}\right\rceil-1,\left\lceil\frac{n-1}{2}\right\rceil,\left\lceil\frac{n-1}{2}\right\rceil,\left\lceil\frac{n-1}{2}\right\rceil+1, \ldots, n-3, n-2, n-1\right)
$$

or,

$$
\left(1,2, \ldots,\left\lfloor\frac{n}{2}\right\rfloor-1,\left\lfloor\frac{n}{2}\right\rfloor,\left\lfloor\frac{n}{2}\right\rfloor,\left\lfloor\frac{n}{2}\right\rfloor+1, \ldots, n-2, n-1\right)
$$

The following lemma shows that the presence of a vertex in a graph with sufficiently many neighbours of degree 1 allows one to determine the graph's weightcolourability by considering a smaller graph instead:

Lemma 3.2.6. Suppose $G$ has a vertex $v$ with a set of leaf neighbours $L$ where $|L| \geq\lceil\operatorname{deg}(v) / 2\rceil$. Let $a \neq b$ be real numbers with $a b>0$. If $G \backslash L$ is $\{a, b\}$-weight colourable, then so is $G$. 
Proof. As mentioned, Proposition 3.2.1 implies that only $a, b \in \mathbb{Z}^{+}$need be considered. Suppose $w$ is an $\{a, b\}$-weight colouring of $G \backslash L$. The possible extensions of $w$ to $G$ give exactly $|L|+1$ possible colours for $v$. Since $a b>0$, the colour of $v$ is different from the colour of the neighbours of $v$ in $L$. Since $v$ has at most $|L|$ neighbours in $G \backslash L$, in at least one of the extensions, the colour of $v$ is different from the colour of the neighbours of $v$ in $G \backslash L$ and so $w$ may be extended as desired.

Corollary 3.2.7. Every tree with at least 3 vertices is $\{a, b\}$-weight colourable, where $a \neq b$ are real numbers with $a b>0$.

Proof. The statement holds for any star, $K_{1, n-1}$, since the assignment of $a$ to all edges achieves the desired result. This implies that the result holds for $n=3$ since the unique tree on 3 vertices is a star. Let $T$ be a tree on $n$ vertices which is not a star and assume the result holds for any tree with fewer than $n$ vertices. Every tree has a vertex $v$ that has at least $\lceil\operatorname{deg}(v) / 2\rceil$ leaf neighbours. Since $T$ is not a star, removing the leaf neighbours of $v$ gives a subtree $T^{\prime}$ on at least 3 vertices. By the induction hypothesis $T^{\prime}$ has an $\{a, b\}$-weight colouring, and so by Lemma 3.2.6, $T$ does as well.

A thread $T=v_{0}, v_{1}, \ldots, v_{t}$ is a walk containing distinct vertices $v_{1}, \ldots, v_{t-1}$ with $\left\{v_{0}, v_{t}\right\} \cap\left\{v_{1}, \ldots, v_{t-1}\right\}=\emptyset$, ends (which are possibly equal) having degree in $G$ at least 3 , and internal vertices with degree in $G$ exactly 2 . If $v_{0}=v_{t}$, then the vertices of $T$ induce a cycle and $v_{0}$ is a cut vertex of the graph. If $v_{0}$ and $v_{t}$ are distinct, then the walk is a path; in this case, the thread is called an ear. If the condition that $\operatorname{deg}_{G}\left(v_{0}\right), \operatorname{deg}_{G}\left(v_{t}\right) \geq 3$ is replaced by $\operatorname{deg}_{G}\left(v_{0}\right), \operatorname{deg}_{G}\left(v_{t}\right) \geq 2$, then a thread and ear become a subthread and subear, respectively. 
The following lemma establishes that long threads may be contracted in a way that maintains weight colourability:

Lemma 3.2.8. Let $G$ be a graph, $P=v_{0} v_{1} \cdots v_{5}$ be a subthread of $G$, and $a \neq b$ be any two real numbers. Let $e_{i}=v_{i} v_{i-1}$ for each $i \in[5]$ and let $G^{\prime}=G /\left\{e_{1}, e_{2}, e_{3}, e_{4}\right\}$.

1. If $w$ is an $\{a, b\}$-weight colouring of $G$, then $w\left(e_{1}\right)=w\left(e_{5}\right) \neq w\left(e_{3}\right)$.

2. If $G^{\prime}$ is $\{a, b\}$-weight colourable, then so is $G$.

3. If $\operatorname{deg}\left(v_{0}\right)=2$ or $\operatorname{deg}\left(v_{5}\right)=2$, then $G$ is $\{a, b\}$-weight colourable if and only if $G^{\prime}$ is $\{a, b\}$-weight colourable.

Proof. 1. If $w\left(e_{1}\right) \neq w\left(e_{5}\right)$, then either one of the two choices for $w\left(e_{3}\right)$ results in an improper colouring between the ends of either $e_{2}$ or $e_{4}$. Hence $w\left(e_{1}\right)=w\left(e_{5}\right)$ and $w\left(e_{3}\right)$ must be distinct.

2. Denote the vertex obtained from the contraction by $v_{0}$; the contraction operation may be considered as replacing the path $P$ by the single edge $v_{0} v_{5}$. If $w^{\prime}$ is an $\{a, b\}$-weight colouring of $G^{\prime}$, then $c^{\prime}\left(v_{0}\right) \neq c^{\prime}\left(v_{5}\right)$. Without loss of generality assume $w^{\prime}\left(v_{0} v_{5}\right)=a$. Let $w(e)=w^{\prime}(e)$ for each $e \notin\left\{e_{1}, e_{2}, e_{3}, e_{4}, e_{5}\right\}$, $w\left(e_{1}\right)=w\left(e_{5}\right)=a$ and $w\left(e_{3}\right)=b$. There are two possibilities for the weights of $e_{2}$ and $e_{4}$. Assigning $w\left(e_{2}\right)=a$ and $w\left(e_{4}\right)=b$ does not yield a proper vertex colouring of $G$ if and only if $c\left(v_{0}\right)=2 a, c\left(v_{5}\right)=a+b$, or both. Similarly, defining $w\left(e_{2}\right)=b$ and $w\left(e_{4}\right)=a$ does not yield a proper vertex colouring of $G$ if and only if $c\left(v_{0}\right)=a+b, c\left(v_{5}\right)=2 a$, or both. Suppose that neither weighting properly colours $V(G)$ by sums. If the first possibility gives $c\left(v_{0}\right)=2 a$, then the second must give $c\left(v_{5}\right)=2 a$. If the first possibility gives $c\left(v_{5}\right)=a+b$, then the second gives $c\left(v_{0}\right)=a+b$. In either case $c\left(v_{0}\right)=c\left(v_{5}\right)$, a contradiction. 
3. Assume, without loss of generality, that $\operatorname{deg}\left(v_{0}\right)=2$ and let $e_{0}$ be the other edge incident with $v_{0}$. Suppose $w$ is an $\{a, b\}$-weight colouring of $G$. By 1 , $w\left(e_{0}\right)=w\left(e_{4}\right)$ and $w\left(e_{1}\right)=w\left(e_{5}\right)$. Hence $c\left(v_{0}\right)=c\left(v_{4}\right) \neq c\left(v_{5}\right)$. Thus, by assigning the common weight of $e_{1}$ and $e_{5}$ to the edge $v_{0} v_{5} \in E\left(G^{\prime}\right)$, an $\{a, b\}$ weight colouring of $G^{\prime}$ is obtained.

The degree condition on the ends of $P$ in Lemma 3.2 .8 (iii) cannot be dropped. For example, by taking $G$ to be the path of length $5, a=1$, and $b=2$, Lemma 3.2 .8 (iii) fails.

Necessary and sufficient conditions for the existence of $\{a, b\}$-weight colourings of cycles are now easily obtained.

Proposition 3.2.9. If $a, b, c \in \mathbb{R}$ are distinct real numbers, then

1. every cycle is $\{a, b, c\}$-weight colourable, and

2. a cycle $C_{n}$ is $\{a, b\}$-weight colourable if and only if $n \equiv 0(\bmod 4)$.

Proof. 1. Bartnicki et al. [15] show that every cycle is weight-colourable from any collection of lists of size 3 assigned to the cycle's edges (this is stated later as Corollary 4.2.4).

2. For any cycle and any edge 2-weighting $w$, if the edges $e_{1}$ and $e_{2}$ are adjacent to a common edge, then $w\left(e_{1}\right) \neq w\left(e_{2}\right)$. By Lemma 3.2 .8 (iii), one only needs to consider the following four cases $-C_{3}, C_{4}, C_{5}$ and $C_{6}$.

(a) Every pair of edges in $C_{3}$ is joined by another edge, and so three edge weights are required. 
(b) If $C_{4}$ has vertices, in order, $v_{1}, v_{2}, v_{3}, v_{4}$, then the weighting $w\left(v_{1} v_{2}\right)=w\left(v_{2} v_{3}\right)=a, w\left(v_{3} v_{4}\right)=w\left(v_{4} v_{1}\right)=b$ gives the proper colouring $c$ with $c\left(v_{1}\right)=c\left(v_{3}\right)=a+b, c\left(v_{2}\right)=2 a$, and $c\left(v_{4}\right)=2 b$.

(c) Let $C_{5}$ have the vertices, in order, $v_{1}, v_{2}, v_{3}, v_{4}, v_{5}$. Without loss of generality, let $w\left(v_{3} v_{4}\right)=a$. If $C_{5}$ is $\{a, b\}$-weight colourable, then $w\left(v_{1} v_{2}\right)=w\left(v_{5} v_{1}\right)=b$, and this in turn implies that $w\left(v_{2} v_{3}\right)=w\left(v_{4} v_{5}\right)=a$. Hence $c\left(v_{3}\right)=2 a=c\left(v_{4}\right)$, a contradiction.

(d) Let $C_{6}$ have the vertices, in order, $v_{1}, v_{2}, v_{3}, v_{4}, v_{5}, v_{6}$. Without loss of generality, let $w\left(v_{1} v_{2}\right)=a$. If $C_{6}$ is $\{a, b\}$-weight colourable, then $w\left(v_{3} v_{4}\right)=b$, and this in turn implies that $w\left(v_{5} v_{6}\right)=a$. Hence $c\left(v_{1}\right)=a+w\left(v_{1} v_{6}\right)=c\left(v_{6}\right)$, a contradiction.

Just as with complete graphs, there exist edge $\{a, b\}$-weightings of $C_{n}$ for $n \not \equiv 0$ $(\bmod 4)$ which yield vertex colourings with only one colouring conflict.

Proposition 3.2.10. For any $a, b \in \mathbb{R}$ and integer $k \geq 1, C_{2 k+1}$ has an edge $\{a, b\}$ weighting $w$ such that only one edge $e=u v$ has the property that $c(u)=c(v)$.

To verify Proposition 3.2.10, we note that Lemma 3.2.8 allows us to only consider $C_{3}$ or $C_{5}$. The "bad" weighting of $C_{5}$ given in the proof of Proposition 3.2.9 gives the colouring stated in Proposition 3.2.10. In the case of $C_{3}$, giving any two edges the same weight and the other edge the other weight achieves the desired colouring.

Proposition 3.2.11. For any $a, b \in \mathbb{R}$ and integer $k \geq 1, C_{4 k+2}$ has an edge $\{a, b\}$ weighting $w$ such that precisely two non adjacent edges at even distance, $e=u v$ and $e^{\prime}=u^{\prime} v^{\prime}$, have the property that $c(u)=c(v)$ and $c\left(u^{\prime}\right)=c\left(v^{\prime}\right)$. Furthermore,

1. e and $e^{\prime}$ may be chosen to be any two edges at an even distance, 
2. the edges adjacent to e must receive the same weight (which may be chosen to be $a$ or $b)$, and

3. the edges adjacent to $e^{\prime}$ must receive the same weight (which may be chosen to be $a$ or $b)$.

Proof. The desired weighting $w$ will be constructed by case analysis. Choose $e$ and $e^{\prime}$ to be any two edges at even distance. Let $f_{1}, f_{2}$ be the edges of the cycle adjacent to $e$. Since $c(u)=c(v)$, it clearly follows that $f_{1}$ and $f_{2}$ must receive the same weight; without loss of generality, let $w\left(f_{1}\right)=w\left(f_{2}\right)=a$ (if one wishes to have $w\left(f_{1}\right)=w\left(f_{2}\right)=b$, then one simply swaps the roles of $a$ and $b$ in the appropriate weighting constructed below).

Suppose the two shortest disjoint paths connecting $e$ and $e^{\prime}$ have length $0(\bmod 4)$. If the edges adjacent to $e^{\prime}$ are to receive the label $a$, then label the edges (in order, beginning with each edge adjacent to $e$ ) according to the pattern $a, b, b, a, a, b, b, a, a, \ldots$ and let $w(e)=w\left(e^{\prime}\right)=a$. If the edges adjacent to $e^{\prime}$ are to receive the label $b$, then label the edges (in order, beginning with each edge adjacent to $e$ ) according to the pattern $a, a, b, b, a, a, b, b, a, a, \ldots$ and let $w(e)=b$ and $w\left(e^{\prime}\right)=a$.

Suppose now that the two disjoint paths connecting $e$ and $e^{\prime}$ have length 2 $(\bmod 4)$. If the edges adjacent to $e^{\prime}$ are to receive the label $a$, then label the edges (in order, beginning with each edge adjacent to $e$ ) according to the pattern $a, a, b, b, a, a, b, b, a, a, \ldots$ and let $w(e)=w\left(e^{\prime}\right)=b$. If the edges adjacent to $e^{\prime}$ are to receive the label $b$, then label the edges (in order, beginning with each edge adjacent to $e$ ) according to the pattern $a, b, b, a, a, b, b, a, a, \ldots$ and let $w(e)=w\left(e^{\prime}\right)=b$.

By applying the proof method of Proposition 3.2.11 to two adjacent edges in the 
cycle $C_{4 k+2}$, the following result is obtained:

Proposition 3.2.12. Let $k$ be an integer, $k \geq 1$. A cycle of length $4 k+2, C_{4 k+2}$, has an edge $\{a, b\}$-weighting such that three consecutive vertices have equal colours and the rest of the cycle is properly coloured. Furthermore, the edge weighting can be chosen so that the weights of the four edges which contribute to the colours of those three vertices will all be $a$, all $b$, or alternate between $a$ and $b$.

Recall that the generalized theta graph $\Theta_{\left(m_{1}, \ldots m_{d}\right)}, d \geq 3$, is the graph constructed from $d$ internally disjoint paths between distinct vertices $x$ and $y$, where the $i^{\text {th }}$ path has of length $m_{i}$. For simplicity, it will be assumed that $m_{1} \leq m_{2} \leq \cdots \leq m_{d}$. The following theorem gives a necessary and sufficient condition for generalized theta graphs to be 2-weight colourable:

Theorem 3.2.13. Let $d \geq 3$ and let $a, b$ be real numbers. The graph $\Theta_{\left(m_{1}, m_{2}, \ldots, m_{d}\right)}$, where $m_{1}, m_{2}, \ldots, m_{d} \in \mathbb{Z}^{+}$, is 2-weight colourable if and only if it is not of the form $\Theta_{\left(1,4 k_{2}+1, \ldots, 4 k_{d}+1\right)}$ with $k_{2}, \ldots, k_{d} \in \mathbb{Z}^{+}$.

Proof. Let $x$ and $y$ be the two vertices of degree greater than two, and let $\left\{P_{i}: 1 \leq i \leq d\right\}$ be the $d$ internally disjoint paths between $x$ and $y$, where $P_{i}$ has length $m_{i}$ for each $1 \leq i \leq d$.

Suppose $w$ is an $\{a, b\}$-weight colouring of $\Theta_{\left(1,4 k_{2}+1, \ldots, 4 k_{d}+1\right)}$. By applying part 1 of Lemma 3.2.8 to each $P_{i}$, the first and last edges of any of the $d$ disjoint paths between $x$ and $y$ must receive same weight. Thus $c(x)=c(y)$, a contradiction since $x$ and $y$ are adjacent. Hence $\Theta_{\left(1,4 k_{2}+1, \ldots, 4 k_{d}+1\right)}$ is not $\{a, b\}$-weight colourable for any $a, b$.

Consider now a generalized theta graph $G=\Theta_{\left(m_{1}, m_{2}, \ldots, m_{d}\right)} ¥ \Theta_{\left(1,4 k_{2}+1, \ldots, 4 k_{d}+1\right)}$. By Lemma 3.2.8, we may consider each $P_{i}$ to be a path of length $1,2,3,4$, or 5 . In 
order to weight the edges so that, for adjacent $u, v \in V(G) \backslash\{x, y\}$, the colour of $u$ is different from the colour of $v$, each $P_{i}$ must be weighted as follows, based on the path's "reduced length" and beginning with the edge incident to $x$ :

- length 1: $a, b$

- length 2: $a a, a b, b a, b b$

- length 3: $a a b, a b b, b a a, b b a$

- length 4: $a a b b, a b b a, b a a b, b b a a$

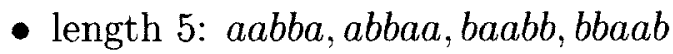

Choose a weighting of the edges based on the above possibilities so that, for each $P_{i}$, every edge incident to $x$ is weighted $a$ (note that there are two such choices for any $P_{i}$ of length at least 2). Call this weighting $w^{\prime}$ and the induced vertex colouring by sums $c^{\prime}$. Certainly, $c^{\prime}(u) \neq c^{\prime}(v)$ for any adjacent $u, v \in V(G) \backslash\{x, y\}$ by our choice of weighting. We may assume, without loss of generality, that $|a| \geq|b|$ (and hence that $a \neq 0)$. Since $d \geq 3$ and $c^{\prime}(x)=d a$, this implies that $c^{\prime}(x) \notin\{2 a, a+b, 2 b\}$. Since $c^{\prime}(v) \in\{2 a, a+b, 2 b\}$ for every $v \in V(G) \notin\{x, y\}$, this in turn implies that $c^{\prime}(x) \neq c^{\prime}(v)$ for any $v \in N_{G}(x) \backslash\{y\}$. Thus, the only possible colouring conflict is if $c^{\prime}(y)=c^{\prime}(v)$ for some $v \in N_{G}(y)$.

We now show that $w^{\prime}$ may be modified to a new weighting $w$, with induced vertex colouring $c$, which gives

- $c(x) \neq c(v)$ for all $v \in N_{G}(x) \backslash\{y\}$ (usually $c(x)=c^{\prime}(x)$ ),

- $c(u) \neq c(v)$ for any adjacent $u, v \in V(G) \backslash\{x, y\}$, and 
- $c(y) \neq c(v)$ for all $v \in N_{G}(y)$

in other words, $c$ properly colours $V(G)$.

Let $n_{j}$ denote the number of paths from $\left\{P_{i}: 1 \leq i \leq d\right\}$ having length congruent to $j(\bmod 4)$. Note that $n_{0}+n_{1}+n_{2}+n_{3}=d=\operatorname{deg}(x)=\operatorname{deg}(y)$. We show how to construct $w$ from $w^{\prime}$ by a case analysis of various values of $n_{0}+n_{2}$, recalling that we may assume that all paths have length $1,2,3,4$, or 5 . Unless explicitly stated otherwise, this is done by taking one or more path $P_{i}$ and swapping the weights assigned to the edges of $P_{i}$ by $w^{\prime}$ to the other possible edge-weighting that begins with $a$ (again, recall that there are two such weightings for each $P_{i}$ with length at least 2).

Case 1: $\mathbf{n}_{\mathbf{0}}+\mathbf{n}_{\mathbf{2}} \geq \mathbf{4}$

For any path $P_{i}$ of even length, swapping from one weighting to the other alters the weight of the edge incident to $y$. There are at least 5 possible values for $c(y)$ if $n_{0}+n_{2} \geq 4$, and so there is a choice of weights $w$ for these such that $c(y) \notin\{2 b, a+b, 2 a, d a\}$.

Case 2: $\mathbf{n}_{\mathbf{0}}+\mathbf{n}_{\mathbf{2}}=\mathbf{3}$

If no $P_{i}$ has length 1 , then $c^{\prime}(y)=d a$ is not forbidden. Since the choice of weights for those $P_{i}$ of even length give 4 possible colours for $c(y)$, there exists a choice such that $c(y) \notin\{2 b, a+b, 2 a\}$.

If $n_{3} \geq 1$, then there is an edge incident to $y$ with weight $b$, since a path $P_{i}$ of length 3 must be weighted $a b b$ or $a a b$; this implies that $c^{\prime}(y) \neq d a$. Again, since the possible weightings of those $P_{i}$ having length 2 and 4 give 4 possible colours for $c(y)$, 
there exists a choice such that $c(y) \notin\{2 b, a+b, 2 a\}$.

Assume that $m_{1}=1$ and that $n_{3}=0$. In the initial weighting, every path $P_{i}$ of length $1(\bmod 4)$ contributes $a$ to $c^{\prime}(y)$. Since there are $d-3$ such $P_{i}$, the possible values of $c^{\prime}(y)$ are $(d-3) a+3 b,(d-2) a+2 b,(d-1) a+b$, and $d a$. If

$$
\{2 b, a+b, 2 a, d a\} \neq\{(d-3) a+3 b,(d-2) a+2 b,(d-1) a+b, d a\}
$$

then there is a valid choice of weightings such that $c$ properly colours $V(G)$ by sums. Suppose that

$$
\{2 b, a+b, 2 a, d a\}=\{(d-3) a+3 b,(d-2) a+2 b,(d-1) a+b, d a\}
$$

This implies that $b=-(d-3) a$. Since $y$ is incident to at least one edge weighted $a$ by $w^{\prime}$ (the edge $x y$ ), we must have that $d-3 \geq 1$, or $d \geq 4$. If $d \geq 5$, then $|b|=(d-3)|a| \geq 2|a|$, contradicting that $|a| \geq|b|$. Hence $d=4$ and so $b=-a$.

We explicitly construct $w$ by weighting $P_{1}, P_{2}, P_{3}, P_{4}$ as follows:

case $\mathrm{i}$. If $n_{0}=3$, then weight the edges of one $P_{i}$ of length $4 a, a,-a,-a$, the other two $a,-a,-a, a$, and let $w(x y)=a$. This gives $c(y)=2 a$ with all neighbours of $y$ having colours in $\{4 a,-2 a, 0\}$.

case ii. If $n_{0}=2$ and $n_{2}=1$ weight the edges of those $P_{i}$ of length 4 with $a, a,-a,-a$, the path of length 2 with $a, a$, and let $w(x y)=a$. This gives $c(y)=0$ with all neighbours of $y$ having colours in $\{4 a,-2 a, 2 a\}$. 
case iii. If $n_{0}=1$ and $n_{2}=2$ weight the edges of the $P_{i}$ of length 4 with $a,-a,-a, a$, those two $P_{i}$ having length 2 with $a,-a$, and let $w(x y)=-a$. This gives $c(x)=2 a$ (a change from $\left.c^{\prime}(x)=4 a\right), c(y)=-2 a$, and all neighbours of $x$ and $y$ (excluding themselves) having colour 0 .

case iv. If $n_{0}=0$ and $n_{2}=3$ weight the edges of those $P_{i}$ having length 2 with $a,-a$. This gives $c(y)=-2 a$ with all neighbours of $y$ having colours in $\{4 a, 0\}$.

Case 3: $\mathbf{n}_{\mathbf{0}}+\mathbf{n}_{\mathbf{2}}=\mathbf{2}$

We first suppose that $n_{3}=0$. If $n_{0}>0$, then assign weights $a, b, b, a$ to the edges of one $P_{i}$ which is length 4 and assign the other $P_{i}$ of even length the weights $a, b$ or $a, a, b, b$ (depending on its length). The colour of $y$ will then be $c(y)=a+b+(d-2) a$. Since $y$ is incident to vertices with colours in $\{d a, a+b\}, c$ is a proper vertex colouring.

Now, suppose that $n_{3}=n_{0}=0$. If $d>3$ or $b \neq 0$, then assign $a, b$ and $a, a$ to the edges of the two $P_{i}$ having length 2 . Weight all $P_{i}$ of length 5 with weights $a, a, b, b, a$, and let $w(x y)=a$ if $x y \in E(G)$. The colour of $y$ will then be $c(y)=a+b+(d-2) a=(d-1) a+b$. Based on the new weighting $w, y$ is incident to vertices with colours in $\{d a, a+b, 2 a\}$. The condition that either $d>3$ or $b \neq 0$ prohibits $(d-1) a+b \in\{a+b, 2 a\}$, and so $c$ is a proper vertex colouring. If $d=3$ and $b=0$, then assign the weights $a, 0$ to each path $P_{i}$ of length 2 , and the single remaining $P_{i}$ (having length $1(\bmod 4)$ ) with $0,0, a, a, 0$ if it has length 5 and 0 otherwise. We then have $c(x)=2 a, c(y)=0$, any neighbour of $x$ which is an internal vertex of some $P_{i}$ has weight 0 or $a$, and any neighbour of $y$ which is an internal vertex of some $P_{i}$ has weight $a$. Hence, $c$ properly colours $V(G)$. 
Assume $n_{3} \geq 1$. Suppose that $n_{0} \neq n_{2}$; choose the initial weighting $w^{\prime}$ so that $c(x)=d a$ and each remaining neighbour of $y$ has colour $a+b$ (note that, for each length $2,3,4,5$ there exists such an edge weighting). This gives $c^{\prime}(y)=a n_{0}+a n_{1}+b n_{2}+b n_{3}$. Since $n_{3} \geq 1$, it follows that $c^{\prime}(x) \neq c^{\prime}(y)$, and so the only possible conflict is if $c^{\prime}(y)=a+b$. In this case change both even length $P_{i}^{\prime}$ 's to their alternate weighting. Call this colouring $c$. Clearly $c(x)=c^{\prime}(x)=d a$, and $c$ produces a new colour at $y$ :

$$
\begin{aligned}
c(y) & =b n_{0}+a n_{1}+a n_{2}+b n_{3} \\
& =c^{\prime}(y)+(a-b)\left(n_{2}-n_{0}\right) \\
& =a+b+(a-b)\left(n_{2}-n_{0}\right) \\
& = \begin{cases}3 a-b & \text { if } n_{0}=0, n_{2}=2 \\
3 b-a & \text { if } n_{0}=2, n_{2}=0\end{cases}
\end{aligned}
$$

In either case, $c(y)=a+b$ implies that $a=b$, a contradiction. If $n_{0}=2$, then all neighbours of $y$ (except perhaps $x$ ) have colour $2 b$, and $3 b-a \neq 2 b$. Similarly if $n_{2}=2$, then the colour at $y$ avoids conflict with its neighbours with colour $2 a$. Hence $c$ is a proper vertex colouring of $G$.

Now, suppose that $n_{3} \geq 1$ and $n_{0}=n_{2}=1$. Again, choose the initial weighting $w^{\prime}$ so that $c^{\prime}(x)=d a$ and each remaining neighbour of $y$ has colour $a+b$. This gives $c^{\prime}(x)=d a \neq\left(n_{1}+1\right) a+\left(n_{3}+1\right) b=c^{\prime}(y)$, and so there is a colouring conflict only if $c(y)=a+b$ or, equivalently, $a n_{1}+b n_{3}=0$. If the edges of $P_{i}$ 's of lengths $1,2,3,4$, and 5 are weighted with $a, a a, a b b, a a b b$, and $a b b a a$, respectively, then

$$
c(y)=a n_{1}+a n_{2}+b n_{3}+b n_{0}=a n_{2}+b n_{0}=a+b \neq d a=c(x),
$$


and no neighbour of $y$ has colour $a+b$. Hence, $c$ properly colours $V(G)$.

Case $4: \mathbf{n}_{\mathbf{0}}+\mathbf{n}_{\mathbf{2}}=\mathbf{1}$

Suppose that $n_{3}=0$. Weight the edges of the path $P_{i}$ having even length $a b$ if it has length 2 and $a a b b$ if it has length 4 . If $x y \in E(G)$, then let $w(x y)=a$. Weight the edges of the path $P_{i}$ having length 5 with aabba. Since $d \geq 3$ and $|a| \geq|b|$, neither $d a$ nor $(d-1) a+b$ can equal $2 a, a+b$, or $2 b$. Since $c(x)=d a$ and $c(y)=b+(d-1) a$, $c$ properly colours $V(G)$.

Suppose that $n_{3} \geq 1$. Choose the initial weighting $w^{\prime}$ so that $c^{\prime}(x)=d a$ and each remaining neighbour of $y$ has colour $a+b$. Note that $w^{\prime}$ assigns the weight $b$ to the edge of the even length $P_{i}$ that is incident to $y$. Since $n_{3} \geq 1$, we have that $c^{\prime}(x)=d a \neq c^{\prime}(y)$. If $c^{\prime}(y) \neq a+b$, then $w^{\prime}$ is an $\{a, b\}$-weight colouring. Suppose that $c^{\prime}(y)=a+b$, or equivalently that

$$
\left(n_{0}+n_{1}-1\right) a+\left(n_{2}+n_{3}-1\right) b=0
$$

We now alter the weight of the even length path to be $b a$ if it has length 2 and baab if it has length 4. Call this weighting $w^{\prime \prime}$ and its induced colouring $c^{\prime \prime}$. Note that $c^{\prime \prime}(x)=(d-1) a+b$ and $c^{\prime \prime}(y) \neq a+b$. All neighbours of $y$ still have colour $a+b$, so the only possible conflicts are between $x$ and its neighbours. A potential conflict falls into one of four cases; in each of these cases we show how $w^{\prime \prime}$ can be altered to give our desired $w$ which properly colours $V(G)$ by sums.

If $c^{\prime \prime}(x)=c^{\prime \prime}(y)$, then $y$ is incident with precisely one edge with weight $b$ since $c^{\prime \prime}(x)=(d-1) a+b$. Since $n_{3} \geq 1$, the edge with weight $b$ comes from a path of length 3. This gives $n_{0}=0, n_{2}=1$ and $n_{3}=1$; Equation 3.2 .1 and $|a| \geq|b|$ gives either 
- $n_{0}=0, n_{1}=1, n_{2}=1, n_{3}=1$ and $b=0$ (case iii. below).

- $n_{0}=0, n_{1}=2, n_{2}=1, n_{3}=1$ and $b=-a$ (case iv. below).

The neighbours of $x$ have accumulated colours either $a+b$ or $2 a$. If $c^{\prime \prime}(x)=(d-1) a+b=a+b$, then this implies that $d=2$ but the hypotheses of the theorem include $d \geq 3$. If $c^{\prime \prime}(x)=(d-1) a+b=2 a$, then $b=-(d-3) a$. The fact that $d \geq 3$ and $|a| \geq|b|$ now give either

- $n_{0}=1, n_{1}=0, n_{2}=0, n_{3}=2$ and $b=0$ which is dealt with in case $\mathbf{i}$. below.

- $n_{0}=1, n_{1}=1, n_{2}=0, n_{3}=2$ and $b=-a$ which is dealt with in case ii. below.

- $n_{0}=0, n_{1}=1, n_{2}=1, n_{3}=1$ and $b=0$ which is dealt with in case iii. below.

- $n_{0}=0, n_{1}=2, n_{2}=1, n_{3}=1$ and $b=-a$ which is dealt with in case iv. below.

Let $w$ be an edge weighting of $G$, given as follows:

case $\mathrm{i}$. In this case $x$ and $y$ are not adjacent. Weight the edges of the path $P_{i}$ of length 4 with bbaa and the two paths of length 3 with $a b b$. Since $b=0$, we have $c(x)=2 a, c(y)=a$, neighbours of $x$ having colours 0 or $a$, and neighbours of $y$ having colour $2 a$ or 0 . Hence, $c$ is a proper vertex colouring.

case ii. In this case $x$ and $y$ are not adjacent. Weight the edges of those $P_{i}$ having length 3 with $a b b$, length 4 with $b b a a$, and length 5 with $a b b a a$. 
Since $b=-a$, we have $c(x)=2 a, c(y)=0$, neighbours of $x$ having colours $-2 a$ or 0 , and neighbours of $y$ having colour $2 a$ or $-2 a$. Hence, $c$ is a proper vertex colouring.

case iii. In this case $x$ and $y$ may be adjacent. If so, weight $x y$ with $a$. Weight the edges of those $P_{i}$ having length 2 with $b b$, length 3 with $b b a$, and length 5 with aabba. Since $b=0$, we have $c(x)=a, c(y)=2 a$, neighbours of $x$ other than $y$ having colours $2 a$ or 0 , and neighbours of $y$ other than $x$ having colour $a$ or 0 . Hence, $c$ is a proper vertex colouring.

case iv. Again, $x$ and $y$ may be adjacent. If so, weight $x y$ with $a$. Weight the edges of those $P_{i}$ having length 2 with $b b$, length 3 with $a b b$, and length 5 with abbaa. Since $b=-a$, we have $c(x)=2 a, c(y)=0$, neighbours of $x$ other than $y$ having colours $-2 a$ or 0 , and neighbours of $y$ other than $x$ having colour $2 a$ or $-2 a$. Hence, $c$ is a proper vertex colouring.

Case 5: $\mathbf{n}_{\mathbf{0}}+\mathbf{n}_{\mathbf{2}}=\mathbf{0}$

Every weighting of the paths $P_{i}$ which gives $c(x)=d a$ must give $c(y)=a n_{1}+b n_{3}$. If $m_{1}=1$, then $n_{3} \geq 1$, since the graph is not $\Theta_{\left(1,4 k_{2}+1, \ldots, 4 k_{d}+1\right)}$; thus $c(x) \neq c(y)$. Suppose $m_{1} \neq 1$. For each $P_{i}$ there are two choices for the colour of $y$ 's neighbour. Each choice leaves $c(y)$ constant. Thus, there is a choice for each path which so that the edge weighting is a proper vertex colouring by sums. 
Having exhausted all cases for $n_{0}+n_{2}$, we have shown that $G$ may be $\{a, b\}$-weight coloured for any $a, b \in \mathbb{R}$.

\subsection{Bipartite graphs}

The property of being $\{a, b\}$-weight colourable is, unfortunately, not closed under subgraph containment, nor is the property of being non- $\{a, b\}$-weight colourable. For example, the graph consisting of $K_{4}$ with a leaf attached is $\{1,2\}$-weight colourable, however $K_{4}$ is not $\{a, b\}$-weight colourable for any choice of $a$ and $b$. Similarly $K_{4}$ contains the subgraph $C_{4}$ which is 2 -weight colourable. This makes it difficult, in general, to determine if a family of graphs $\mathcal{G}$ that is closed under subgraph containment has the property that every $G \in \mathcal{G}$ is 2 -weight colourable.

For a pair of real numbers $a, b \in \mathbb{R}$, let $\mathcal{H}_{a, b}$ be the set of graphs which are not $\{a, b\}$-weight colourable. We show in this section that is possible to characterize the minimal graphs of $\mathcal{H}_{a, b}$ with respect to subgraph containment for many pairs $\{a, b\}$, most notably for the pair $\{1,2\}$. To be precise, Theorem 3.3.8 states that any graph which is not $\{1,2\}$-weight colourable (among other sets $\{a, b\}$ ) must contain $C_{2 k+1}$ or $C_{4 k+2}$ as a subgraph for some positive integer $k$.

A graph $G$ is round if every cycle of $G$ has length $0(\bmod 4)$. Note that the class of round graphs is much richer than those trivially obtained by taking a graph and subdividing each edge into a path of length 4 ; for example, $\Theta_{(2,2,2)} \cong K_{2,3}$ is a round graph which is not obtained in this way. Round graphs are a particular case of a larger class of graphs studied by Akbari et al. in [6], where they determine the choosability of graphs whose cycles have length a multiple of some fixed integer $k \geq 3$.

The following lemma establishes a useful subgraph property of round graphs: 
Proposition 3.3.1. If $G$ is a round graph and $\Theta_{(i, j, k)}$ is a subgraph of $G$, then $i, j$ and $k$ are even and $i \equiv j \equiv k(\bmod 4)$.

Proof. Let $\Theta_{(i, j, k)}$ be a subgraph of $G$ and let $P_{i}, P_{j}$ and $P_{k}$ be the corresponding paths of length $i, j$ and $k$, respectively. Since $G$ is round, $i+j \equiv i+k \equiv j+k \equiv 0$ $(\bmod 4)$. The result follows.

Given an edge $\{a, b\}$-weighting of a graph, the colour of every vertex is of the form $r a+s b$ for some nonnegative integers $r, s$. A vertex is called $a$-even ( $a$-odd) if its colour is $r a+s b$ with $r$ even (odd). Note that the $a$-parity of a vertex does not necessarily refer to the parity of the colour of the vertex. By Proposition 3.2.1, if $a$ and $b$ are not independent over $\mathbb{Q}$, then they may be assumed to be relatively prime integers and in particular one may be assumed to be odd (without loss of generality, say $a$ is odd in this case). If $b$ is even, which will be the case in a number of the following results, then the $a$-parity of a vertex does, in fact, coincide with the parity of its colour.

A number of the arguments which follow rely on this notion of $a$-parity. For instance, in some cases $G$ is shown to be $\{a, b\}$-weight colourable only for values of $a, b$ whose ratio cannot be reduced to a ratio of odd integers. Also occasionally excluded from consideration are those sets $\{a, b\}$ for which $a$ and $b$ do not have the same sign or which have one element equal to zero. Let the sets $\mathcal{E}$ and $\mathcal{N}$ be defined as follows:

$$
\begin{aligned}
& \mathcal{E}=\left\{\begin{array}{l|l}
\{a, b\} & \left.\frac{a}{b}=\frac{p}{q}, p, q \text { odd integers }\right\}
\end{array}\right. \\
& \mathcal{N}=\left\{\begin{array}{l|l}
\{a, b\} & \left.\frac{a}{b}=\frac{p}{q}, p, q \in \mathbb{Z}, p q \leq 0\right\}
\end{array}\right.
\end{aligned}
$$


We have already seen examples of bipartite graphs which are 2-weight colourable $\left(C_{4 k}\right.$ for any $k \geq 1$, bipartite theta graphs except $\left.\Theta_{\left(1,4 k_{2}+1, \ldots, 4 k_{d}+1\right)}\right)$ and some which are not $\left(C_{4 k+2}\right.$ for any $\left.k \geq 1\right)$. From these examples, it can be seen that a bipartite graph $G$ with both parts of odd size is not necessarily $\{a, b\}$-weight colourable. However, if $G$ has one part of even size, it can be shown that $G$ is $\{a, b\}$-weight colourable for particular values of $a$ and $b$. This generalizes the bipartite case of Duan et al.'s result that $\chi_{\Sigma}^{e}(G) \leq k$ if $G$ has a proper $k$-colouring where one colour class has even size $[40]$.

Theorem 3.3.2. Let $a, b \in \mathbb{R}$ be such that $\{a, b\} \notin \mathcal{E}$. If $G$ is a connected bipartite graph with at least one part being of even size, then $G$ is $\{a, b\}$-weight colourable. Furthermore, if $(X, Y)$ is the bipartition of $V(G)$ into independent sets with $|X|$ even, then the weighting may be chosen so that $x$ is a-odd for every $x \in X$ and $y$ is a-even for every $y \in Y$.

Proof. By Corollary 3.2.2 and since $\{a, b\} \notin \mathcal{E}$, we may assume that $a$ is an odd integer and $b$ is an even integer. Assign the weight $b$ to each edge of $G$. Clearly $v$ is $a$-even for each $v \in V(G)$. Let $X=\left\{x_{1}, x_{2} \ldots x_{2 k}\right\}$ and let $P_{i}$ be an $x_{2 i-1} x_{2 i}$-path in $G$. By changing every edge weight along $P_{1}$, only the $a$-parity of $x_{1}$ and $x_{2}$ are changed. By repeating this process for each $P_{i}$, every vertex of $X$ will be $a$-odd and every vertex of $Y$ will be $a$-even.

Call the resulting edge weighting $w$. If $w$ is not an $\{a, b\}$-weight colouring, then there are adjacent vertices $x$ and $y$ such that $c(x)=c(y)$. This implies that there exist integers $r, r^{\prime}, s, s^{\prime}$ such $c(x)=r a+s b$ where $r$ is odd, $c(y)=r^{\prime} a+s^{\prime} b$ where $r^{\prime}$ is even, and $r a+s b=r^{\prime} a+s^{\prime} b$. If $a$ and $b$ are linearly independent over $\mathbb{Q}$, then $r=r^{\prime}$, a contradiction. Hence $b=(p / q) a$ for some $p, q \in \mathbb{Z}$ with $\operatorname{gcd}(p, q)=1$. Thus 
$r q+s p=r^{\prime} q+s^{\prime} p$. If $p$ is even, then the fact that $r$ is odd and $r^{\prime}$ is even implies that $q$ is even, a contradiction. If $p$ is odd, then our choice of $a$ odd and $b$ even implies that $a p \neq b q$, a contradiction. Therefore, no such $p, q \in \mathbb{Z}$ exist, so no adjacent vertices $x, y$ with $c(x)=c(y)$ exist, and thus $w$ is an $\{a, b\}$-weight colouring of $G$.

Corollary 3.3.3. Let $a, b \in \mathbb{R}$ be such that $\{a, b\} \notin \mathcal{E} \cup \mathcal{N}$. If $G ¥ K_{2}$ is a connected bipartite graph with a vertex of degree 1 , then $G$ is $\{a, b\}$-weight colourable.

Proof. Let $V(G)=X \cup Y$ be a bipartition of the vertices of $G$. Let $x \in X$ be a vertex of degree 1 and let $y \in Y$ be its neighbour. If $|X|$ or $|Y|$ is even, then $G$ is $\{a, b\}$-weight colourable by Theorem 3.3.2. If $|X|$ is odd, then $G-x$ has an $\{a, b\}$ weight colouring by Theorem 3.3.2, say $w^{\prime}$, such that vertices in $X \backslash\{x\}$ are $a$-odd and vertices in $Y$ are $a$-even. By assigning $b$ to the edge $x y$, the $a$-parity of all the vertices is maintained. Also, since $\{a, b\} \notin \mathcal{N}, c^{\prime}(y) \neq 0$ and so $x$ and $y$ will receive different colours, thus giving an $\{a, b\}$-weight colouring of $G$.

Note that this implies that trees are $\{a, b\}$-weight colourable if $\{a, b\} \notin \mathcal{E} \cup \mathcal{N}$, a result which we already know from Corollary 3.2.7.

We now move to our study of round graphs, showing in Theorem 3.3.7 that round graphs are $\{a, b\}$-weight colourable for many sets $\{a, b\}$, notably $\{1,2\}$.

Theorem 3.3.4. Let $a, b \in \mathbb{R}$ be such that $\{a, b\} \notin \mathcal{E} \cup \mathcal{N}$. Let $G$ be a connected bipartite graph with a thread $P$ of even length and let $U$ be the set of internal vertices of $P$. If $G-U$ is connected, then $G$ is $\{a, b\}$-weight colourable.

Proof. Assume, without loss of generality, that $a$ is a positive odd integer and $b$ is a positive even integer. If $X \cup Y$ is the bipartition of $V(G)$ and either $|X|$ or $|Y|$ is 
even, then $G$ is $\{a, b\}$-weight colourable by Theorem 3.3.2. Assume both parts of $G$ are of odd size. Let $x$ and $y$ be the ends of $P$.

Case 1: $x$ and $y$ are distinct

By Lemma 3.2.8, $P$ may be assumed to be a path of length either 2 or 4 .

Suppose that $P$ is of length 2 ; say $P=x v y$. Let $G^{\prime}$ be the bipartite graph obtained from $G$ by deleting $v$ and adding two leaves, $v_{1}$ adjacent to $x$ and $v_{2}$ adjacent to $y$. Now $G^{\prime}$ is connected and bipartite with an even part, where $v_{1}$ and $v_{2}$ both belong to the even part. Theorem 3.3.2 states that there exists an $\{a, b\}$-weight colouring of $G^{\prime}$, say $w^{\prime}$, so that $v_{1}$ and $v_{2}$ are both $a$-odd vertices. It follows that $x v_{1}$ and $y v_{2}$ must both receive $a$ as their weight. Let $w$ be an edge $\{a, b\}$-weighting of $G$, where $w(x v)=w^{\prime}\left(x v_{1}\right)=a, w(y v)=w^{\prime}\left(y v_{2}\right)=a$ and $w(e)=w^{\prime}(e)$ for $e \in E(G) \backslash\{x v, y v\}$. If $w$ is not an $\{a, b\}$-weight colouring of $G$, then either $c(x)=2 a$ or $c(y)=2 a$. Without loss of generality, suppose $c(x)=r a+s b=2 a$ (a similar argument will hold for $y$ ). Since $w(x v)=a$ and $r$ is even, it follows that $r \geq 2$. If $r=2$, then $s b=0$. This implies that $s=0$ or, equivalently, that $\operatorname{deg}_{G}(x)=2$, a contradiction. If $r \geq 3$, then $s b<0$. This implies that $b<0$, a contradiction. Thus $w$ is an $\{a, b\}$-weight colouring of $G$.

Suppose that $P$ has length 4 , and let $P=x v_{1} v_{2} v_{3} y$. The graph $G^{\prime}=G-v_{2}$ is bipartite with an even side $X^{\prime}$, and $x, y \in X^{\prime}$. Theorem 3.3 .2 states that there exists an $\{a, b\}$-weight colouring of $G^{\prime}$, say $w^{\prime}$, such that $v_{1}$ and $v_{3}$ are both $a$-even vertices. It follows that $x v_{1}$ and $y v_{3}$ must both receive $b$ as their weight. Let $w$ be an edge $\{a, b\}$-weighting of $G$ such that $w\left(v_{1} v_{2}\right)=w\left(v_{2} v_{3}\right)=a$ and $w(e)=w^{\prime}(e)$ for $e \in E(G) \backslash\left\{v_{1} v_{2}, v_{2} v_{3}\right\}$. If $w$ is not an $\{a, b\}$-weight colouring of $G$, then either $c(x)=a+b$ or $c(y)=a+b$. If $c(x)=r a+s b=a+b$, then $(r-1) a=-(s-1) b$, 
and hence $r$ is odd. Since $a$ and $b$ are positive integers, either $r-1<0$ or $s-1<0$. Since $w\left(x v_{1}\right)=b, s$ is nonzero. Since $r$ is odd, $r \neq 0$ as well, a contradiction. Thus $w$ is an $\{a, b\}$-weight colouring of $G$.

Case 2: $x$ and $y$ are not distinct

Call this vertex $x$. The thread $P$ is a cycle which is an end block of $G$ and $x$ is a cut vertex of $G$. Let $z_{1}$ and $z_{2}$ be the neighbours of $x$ in $P$. Since $G^{\prime}=G-U$ is a connected bipartite graph with one part having even size, Theorem 3.3 .2 says that there is an $\{a, b\}$-weight colouring of $G^{\prime}$, say $w^{\prime}$. Let $w^{\prime \prime}$ be an edge weighting of $P$, defined as follows:

- If $P$ has length $2(\bmod 4)$, then by Proposition 3.2 .12 there exists an $\{a, b\}$ weight colouring of $P, w^{\prime \prime}$, so that $c^{\prime \prime}\left(z_{1}\right)=c^{\prime \prime}(x)=c^{\prime \prime}\left(z_{2}\right)=2 a$ and $P$ is properly coloured elsewhere.

- If $P$ has length $0(\bmod 4)$, then by Proposition 3.2 .9 there exists an edge $\{a, b\}$ weighting of $P, w^{\prime \prime}$, which properly colours $P$. Furthermore, since the colours of the vertices of a cycle will be $2 a, 2 b$, or $a+b, w^{\prime \prime}$ may be chosen so that $c^{\prime \prime}(x)$ the larger of $2 a$ and $2 b$.

Let $w$ be the weighting obtained by combining $w^{\prime}$ and $w^{\prime \prime}$. Since $c(x)>c\left(z_{1}\right)$, $c(x)>c\left(z_{2}\right)$ and $x$ has the same $a$-parity under $w$ as under $w^{\prime}$, the colour of $x$ must be distinct from its neighbours in $G$. Since all other vertices are properly coloured by $w^{\prime}$ or $w^{\prime \prime}, w$ gives an $\{a, b\}$-weight colouring of $G$.

Theorem 3.3.5. If $G$ is a 2-connected round graph which is not a cycle, then $G$ contains at least 2 even ears. 
Proof. We first claim that $G$ contains no 2-connected proper subgraph which contains all even ears of $G$. Suppose, to the contrary, that $H$ is a maximal 2-connected proper subgraph of $G$ that contains all even ears of $G$. There exist two vertices of $H$, say $x$ and $y$, which are connected by a path $P$ such that $H \cap P=\{x, y\}$.

Since $H$ is 2-connected, there are also 2 internally disjoint paths $P^{\prime}$ and $P^{\prime \prime}$ in $H$ between $x$ and $y$. Thus $P \cup P^{\prime} \cup P^{\prime \prime}$ is a theta graph and, by Lemma 3.3.1, $P$ must be of even length. Since $H$ already contains all even ears of $G, H^{\prime}=H \cup P$ is a proper 2-connected subgraph of $G$, which contradicts the maximality of $H$.

If $G$ has no even ear, then any cycle of $G$ is a 2-connected subgraph containing all the even ears, a contradiction since $G$ is not a cycle. If $G$ has only one ear, let $T$ be the ear and let $x$ and $y$ be the ends of $T$. There are two internally disjoint paths connecting $x$ and $y$, one of which must be internally disjoint from $T$. This path together with $T$ forms a cycle that contains all the even ears of $G$, a contradiction.

Corollary 3.3.6. If $G$ is a round graph and all threads of $G$ are odd, then $G$ has at least two leaves.

We are now able to prove the major result of this section.

Theorem 3.3.7. Every round graph is $\{a, b\}$-weight colourable for $\{a, b\} \notin \mathcal{E} \cup \mathcal{N}$.

Proof. Let $G$ be a round graph. Let $B$ be an end block with vertex of attachment $v$. If $B$ is isomorphic to $K_{2}$, then $G$ is a bipartite graph with a leaf and thus is $\{a, b\}$ weight colourable by Corollary 3.3.3. If $B$ is a cycle, then $B$ is an even thread and $G$ is $\{a, b\}$-weight colourable by Theorem 3.3.4. Otherwise, if $B$ is a 2-connected graph which is not a cycle, then by Theorem 3.3.5, $B$ has at least two even ears and thus $B$ has at least one even ear, say $P$, which does not contain $v$ as an internal vertex. 
Let $U$ be the internal vertices of $P$. Since $G-U$ is connected, $G$ is $\{a, b\}$-weight colourable by Theorem 3.3.4.

As stated in the introduction of the section, Theorem 3.3.7, together with Proposition 3.2 .9 , gives a class of minimal subgraphs with respect to containment which cannot be $\{a, b\}$-weight coloured for $\{a, b\} \notin \mathcal{E} \cup \mathcal{N}$.

Corollary 3.3.8. Let $a$ and $b$ be real numbers such that $\{a, b\} \notin \mathcal{E} \cup \mathcal{N}$. Any graph which is not $\{a, b\}$-weight colourable must contain a cycle of length 1,2 or $3(\bmod 4)$.

We end this section with the following problem, proposed by myself along with Khatirinejad, Naserasr, Newman, and Stevens in [66]; a similar question was independently posed by Lu, Yang and Zhang in [70] for $\{1,2\}$-weight colourings.

Problem 3.3.9. Is it true that all bipartite graphs except $C_{4 k+2}$ and $\Theta_{\left(1,4 k_{1}+1,4 k_{2}+1, \ldots, 4 k_{d}+1\right)}$ are 2 -weight colourable?

\subsection{Families of 2-weight colourable graphs}

The previous section established a number of examples of $\{a, b\}$-weight colourable graphs for a wide array of values of $a$ and $b$. However we have seen few examples of graphs for which $a$ and $b$ can be any distinct real numbers. The Petersen graph is an example of a 2-weight colourable graph, as shown in Figure 2 on page 73.

By Proposition 3.2.3, note that any 2-weight colouring of the Petersen graph gives a proper 3-colouring of it, which is an optimal proper vertex colouring.

In this section, more families of 2 -weight colourable graphs are given, as is a class of graphs which is $\{a, b\}$-weight colourable when $a b>0$. In particular, unicyclic graphs and Cartesian products of graphs will be studied. 


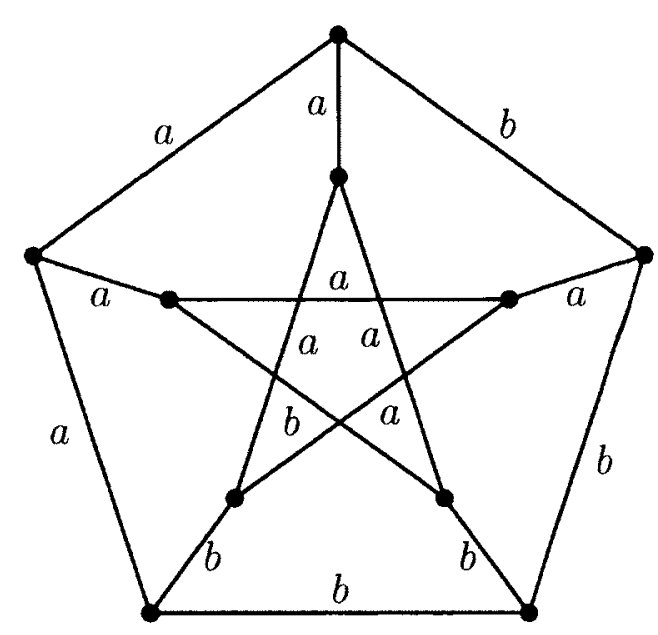

Figure 2: An $\{a, b\}$-weight colouring of the Petersen graph

Theorem 3.4.1. Every connected unicyclic graph except $C_{2 k+1}$ and $C_{4 m+2}$ is $\{a, b\}$ weight colourable, where $a$ and $b$ are real numbers with $a b>0$.

Proof. Assume, without loss of generality, that $0<a<b$. Suppose the theorem is false, and let $G$ be the counterexample on the fewest number of vertices. Let $C$ be the only cycle of $G$. By Lemma 3.2.6, every vertex of $G$ may be assumed to be on $C$ or adjacent to a vertex of $C$. Every vertex of $G$ may also be assumed to have degree at most 3. Suppose that $C$ has only one vertex of degree 3 , say $v$. Let $x$ and $y$ be the neighbours of $v$ on $C$. By Propositions 3.2.10 and 3.2.12, there exists an edge weighting $w$ of $C$ which yields a proper colouring on $C-v$ and $c(v) \geq c(x), c(y)$. By assigning $b$ to the other edge incident with $v$, we get an $\{a, b\}$-weight colouring of $G$, a contradiction.

Next, we claim that $G$ has at most one ear of length at least 2. Suppose not; choose a maximal path of degree 3 vertices on $C, x_{1} x_{2} \cdots x_{k}$, and remove all leaves of $G$ adjacent to those vertices. Call this subgraph $G^{\prime}$. By minimality of $G$, there 
exists an $\{a, b\}$-weight colouring $w^{\prime}$ of $G^{\prime}$. Let $w$ be the weighting of $E(G)$ given by $w(e)=w^{\prime}(e)$ if $e \in E\left(G^{\prime}\right)$ and $w(e)=b$ otherwise. The only possible conflicts are between $x_{1}$ and its neighbour on $C$ which is not $x_{2}$, say $y$ (or, similarly, between $x_{k}$ and it's neighbour on $C$ which is not $\left.x_{k-1}\right)$. However, since $c\left(x_{1}\right) \geq a+b+w\left(x_{1} y\right)$ and $c(y) \leq w\left(x_{1} y\right)+b$ (similar for $\left.x_{k}\right), w$ is an $\{a, b\}$-weight colouring of $G$ which contradicts the choice of $G$.

Suppose $G$ has exactly one ear of length at least 2. Let $e=r s$ and $e^{\prime}=r^{\prime} s^{\prime}$ be the two edges that have exactly one endpoint of degree 2; without loss of generality, let $\operatorname{deg}(r)=\operatorname{deg}\left(r^{\prime}\right)=2, \operatorname{deg}(s)=\operatorname{deg}\left(s^{\prime}\right)=3$. Note that $r$ and $r^{\prime}$ need not be distinct, while $s$ and $s^{\prime}$ are necessarily distinct since there are at least 2 vertices on $C$ of degree 3. We construct an $\{a, b\}$-weight colouring of $G$ based on the length of $C(\bmod 4)$.

- Suppose $|C|$ is odd. By Proposition 3.2.10, $C$ has an $\{a, b\}$-edge weighting $w^{\prime}$ which gives a proper vertex colouring except across rs. Let $w(e)=w^{\prime}(e)$ if $e \in E(C)$. If $c^{\prime}\left(r^{\prime}\right)-c^{\prime}\left(s^{\prime}\right)=a$, let $w(e)=b$ for all $e \in E(G) \backslash E(C)$. Otherwise, let $w(e)=a$ for all $e \in E(G) \backslash E(C)$. Clearly each leaf's neighbour has a colour strictly greater than its own. Since $w^{\prime}$ gives a proper colouring of $C$ except for $r$ and $s$, the only adjacent vertices of $G$ which might not be properly coloured are $r$ and $s$ or $r^{\prime}$ and $s^{\prime}$. However, the choice of colours available for the leaves of $G$ guarantees that $r, s, r^{\prime}, s^{\prime}$ are properly coloured as well. Thus $w$ is an $\{a, b\}$-weight colouring of $G$.

- Suppose $|C| \equiv 0(\bmod 4)$. By Proposition 3.2.9, $C$ has an $\{a, b\}$-weight colouring $w^{\prime}$ such that $c(r)=2 a$ and $c(s)=a+b$. Let $w(e)=w^{\prime}(e)$ if $e \in E(C)$. If $c^{\prime}\left(r^{\prime}\right)-c^{\prime}\left(s^{\prime}\right)=a$, let $w(e)=b$ for $e \in E(G) \backslash E(C)$. Otherwise, let $w(e)=a$ for $e \in E(G) \backslash E(C)$. By the argument above, $G$ is $\{a, b\}$-weight coloured by $w$. 
- Suppose $|C| \equiv 2(\bmod 4)$. Let $t$ be the other neighbour of $s$ on $C$ (note that $t$ may be equal to $s^{\prime}$ ) and let $t^{\prime}$ be the other neighbour of $r^{\prime}$ on $C$. By Proposition 3.2.12, there is an $\{a, b\}$-edge weighting such that all vertices are properly coloured except $r, s$ and $t$, and such that $w\left(t^{\prime} r^{\prime}\right)=w\left(r^{\prime} s^{\prime}\right)=a$. Let $w(e)=w^{\prime}(e)$ for all $e \in E(C)$. Let $f$ be the edge between $s$ and its leaf, and let $w(f)=a$. For each $e \in E(G) \backslash E(C) \backslash\{f\}$, let $w(e)=b$. The only possible improperly coloured pairs of vertices are $r$ and $s, s$ and $t$ or $r^{\prime}$ and $s^{\prime}$. However,

$$
\begin{aligned}
c(r) & =c^{\prime}(r)=c^{\prime}(s)<c(s) \\
c(s) & =c^{\prime}(s)+a=c^{\prime}(t)+a<c^{\prime}(t)+b=c(t) \\
c\left(r^{\prime}\right) & =2 a<a+2 b=c\left(s^{\prime}\right)
\end{aligned}
$$

and so $w$ is an $\{a, b\}$-weight colouring of $G$.

The only remaining case is that every vertex of $C$ has degree 3 . If $|C|$ is even, assign the same weight to all the edges on the cycle and alternating weights to the leaf edges. The reader can verify that a solution for the cases when $|C|=3$ or $|C|=5$ exists. Each of these cases can be extended to larger odd cycle by making the replacement indicated in Figure 3. Note that the variables $\bar{\ell}$ and $\bar{n}$ refer to the weights different from $\ell$ and $n$, respectively.
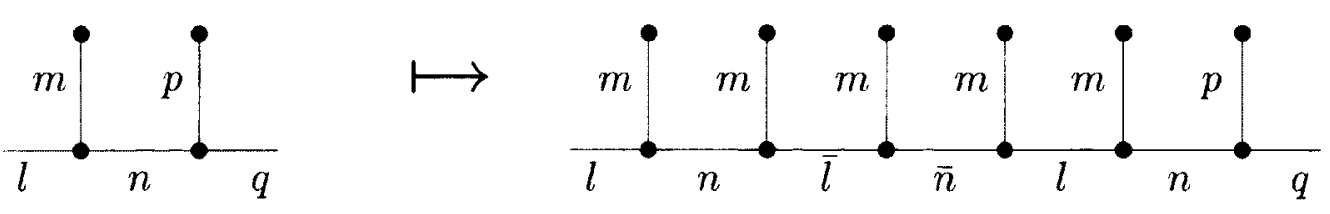

Figure 3: Replacement operation to expand 2-weight colourings to larger cycles. 
Thus, no minimal counterexample $G$ exists.

We now turn our attention to Cartesian products of graphs, showing that many products are 2-weight colourable graphs.

Proposition 3.4.2. For $n \geq 4$, the graph $K_{2} \square K_{n}$ is 2-weight colourable.

Proof. Let $K_{n}$ and $K_{n}^{\prime}$ be the two copies of the complete graph and let

$$
\begin{aligned}
V\left(K_{n}\right) & =\left\{u_{1}, u_{2}, \ldots, u_{\lfloor n / 2\rfloor}, v_{\lfloor n / 2\rfloor}, v_{\lfloor n / 2\rfloor+1}, \ldots, v_{n-2}, v_{n-1}\right\} \\
V\left(K_{n}^{\prime}\right) & =\left\{u_{1}^{\prime}, u_{2}^{\prime}, \ldots, u_{\lfloor n / 2\rfloor}^{\prime}, v_{\lfloor n / 2\rfloor}^{\prime}, v_{\lfloor n / 2\rfloor+1}^{\prime}, \ldots, v_{n-2}^{\prime}, v_{n-1}^{\prime}\right\}
\end{aligned}
$$

Let $p$ be a derangement (i.e. a permutation with no fixed points) of $[1,\lfloor n / 2\rfloor]$ and $\pi$ be a derangement of $[\lfloor n / 2\rfloor, n-1]$. Let $u_{i}$ be adjacent to $u_{p(i)}^{\prime}$ for all $1 \leq i \leq\lfloor n / 2\rfloor$ and $v_{i}$ be adjacent to $v_{\pi(i)}^{\prime}$ for $\lfloor n / 2\rfloor \leq i \leq n-1$.

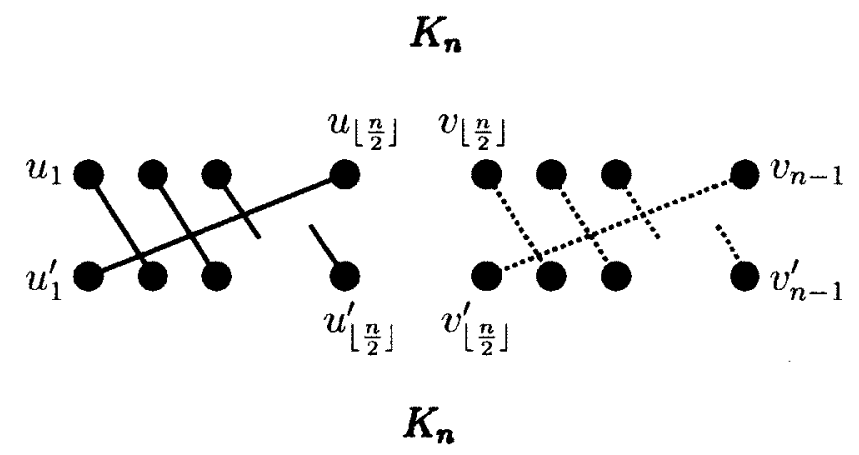

Figure 4: An $\{a, b\}$-weight colouring of $K_{2} \square K_{n}$

Since the graph is $n$-regular, if adjacent vertices have distinct colours, then they have distinct numbers of incident edges having weight $b$. By Lemma 3.2.5, there exist edge weightings of $K_{n}$ and $K_{n}^{\prime}$ so that the subscript of the vertex is precisely equal to 
the number of edges weighted $b$ incident to that edge in $K_{n}$. Label $u_{i} u_{p(i)}^{\prime}$ with $a$ for all $1 \leq i \leq\lfloor n / 2\rfloor$ and weight $v_{i} v_{\pi(i)}^{\prime}$ with $b$ for $\lfloor n / 2\rfloor \leq i \leq n-1$ (Figure 4 on page 76 gives an illustration of this construction where the dotted edges receive weight $b$ ). Any two vertices that are adjacent have a distinct number of incident edges weighted $b$ and thus $K_{2} \square K_{n}$ is 2-weight colourable.

Proposition 3.4.3. The graph $K_{2} \square C_{n}$ is 2-weight colourable if and only if $n \geq 4$ and $n \neq 5$.

Proof. If $n$ is even, then give every edge of one copy of $C_{n}$ weight $a$ and every edge of the other copy weight $b$. By alternating the weights of the images of $K_{2}$ between $a$ and $b$ along the cycles, the desired $\{a, b\}$-weight colouring is obtained.

An example of an $\{a, b\}$-weight colouring of $K_{2} \square C_{7}$ is given in Figure 5; dotted edges are to receive weight $b$.

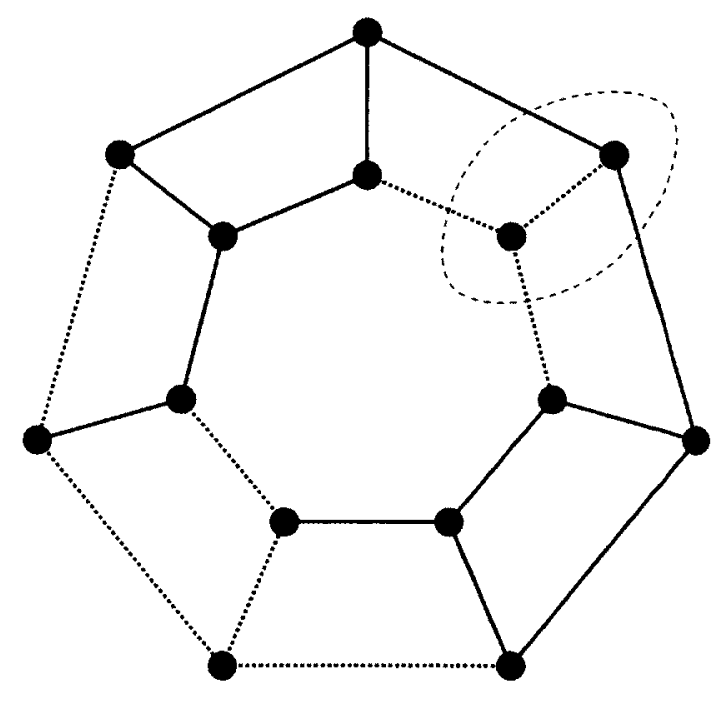

Figure 5: An $\{a, b\}$-weight colouring of $K_{2} \square C_{7}$

The $\{a, b\}$-weight colouring given for $K_{2} \square C_{7}$ can be extended to an $\{a, b\}$-weight 
colouring of $K_{2} \square C_{9}$ by the replacement operation shown in Figure 6. Note that the right subgraph contains the left one, and thus this operation may be repeated as many times as needed to give an $\{a, b\}$-weight colouring for any $K_{2} \square C_{2 k+1}(k \geq 3)$.

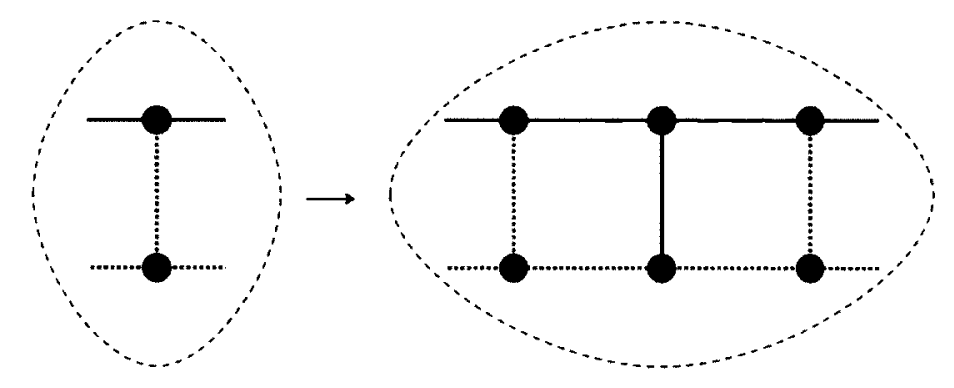

Figure 6: Replacement operation for $\{a, b\}$-weight colourings of $K_{2} \square C_{2 k+1}$

We now show that $K_{2} \square C_{3}$ has no $\{a, b\}$-weight colouring for any choice of $a$ and b. Let $G=(V, E)$ have vertex and edge sets as follows:

$$
\begin{gathered}
V(G)=\left\{x_{1}, x_{2}, x_{3}, y_{1}, y_{2}, y_{3}\right\} \\
E(G)=\left\{x_{1} x_{2}, x_{2} x_{3}, x_{3} x_{1}, y_{1} y_{2}, y_{2} y_{3}, y_{3} y_{1}, x_{1} y_{1}, x_{2} y_{2}, x_{3} y_{3}\right\}
\end{gathered}
$$

Since two of $x_{1} y_{1}, x_{2} y_{2}, x_{3} y_{3}$ will receive the same weight, neither of the cycles $x_{1} x_{2} x_{3} x_{1}$ or $y_{1} y_{2} y_{3} y_{1}$ may be monochromatic in terms of $a$ or $b$. Assume, without loss of generality, that $w\left(x_{1} x_{2}\right)=w\left(x_{2} x_{3}\right)=a$ and $w\left(x_{3} x_{1}\right)=b$ (see Figure 7 on page 79 ).

The edges $y_{1} y_{2}$ and $y_{2} y_{3}$ cannot both have weight $a$, since this would give $c\left(x_{2}\right)=c\left(y_{2}\right)$. If $w\left(y_{1} y_{2}\right)=w\left(y_{2} y_{3}\right)=b$, then $w\left(x_{1} y_{1}\right) \neq w\left(x_{3} y_{3}\right)$ (else $\left.c\left(y_{1}\right)=c\left(y_{3}\right)\right)$. Without loss of generality, suppose that $w\left(x_{1} y_{1}\right)=a$ and $w\left(x_{3} y_{3}\right)=b$. This forces $w\left(y_{1} y_{3}\right)=b$. It may be checked that any choice of $w\left(x_{2} y_{2}\right)$ creates a conflict at this point. Now, suppose that only one of $y_{1} y_{2}$ and $y_{2} y_{3}$ is weighted $a$, say $w\left(y_{1} y_{2}\right)=b$ and $w\left(y_{2} y_{3}\right)=a$. If $w\left(y_{3} y_{1}\right)=a$, then $c\left(x_{1}\right)=c\left(y_{1}\right) ;$ if $w\left(y_{3} y_{1}\right)=b$, then $c\left(x_{3}\right)=c\left(y_{3}\right)$. Hence $K_{2} \square C_{3}$ has no $\{a, b\}$-weight colouring. 


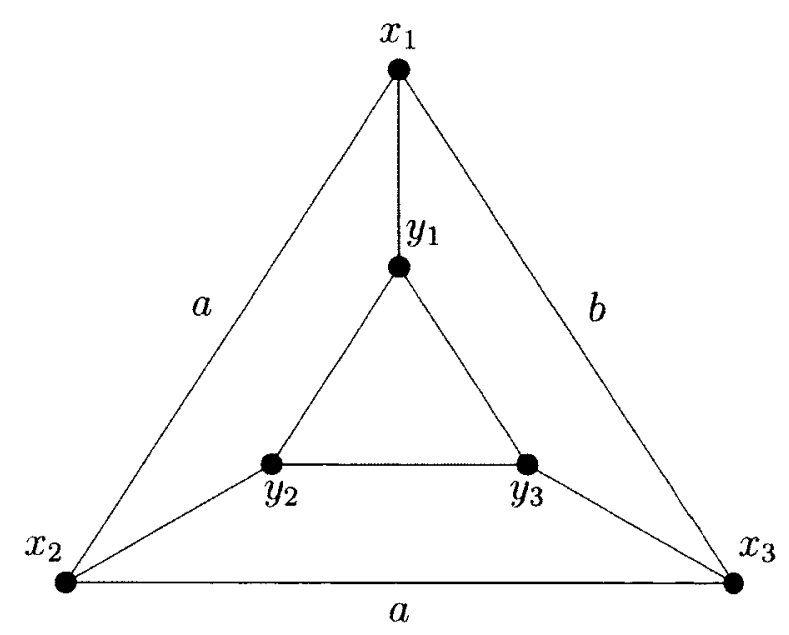

Figure 7: A partial edge $\{a, b\}$-weighting of $K_{2} \square C_{3}$

A similar (and much longer) analysis can show that no $\{a, b\}$-weight colouring of $K_{2} \square C_{5}$ exists.

Theorem 3.4.4. Let $G$ be a graph and $H$ be a regular bipartite graph. If $G \square K_{2}$ is 2-weight colourable, then $G \square H$ is 2-weight colourable.

Proof. Let $w$ be an $\{a, b\}$-weight colouring of $G \square K_{2}$. Denote the two copies of $G$ by $G_{1}$ and $G_{2}$ and denote the vertices of $K_{2}$ by $t_{1}$ and $t_{2}$. Since $H$ is regular (say $d$-regular) and bipartite, Hall's Theorem guarantees a perfect matching $M$ of $H$. Let $X$ and $Y$ be the parts of $V(H)$.

Define an edge weighting of $G \square H$ as follows. For each edge $e=x y \in M$ where $x \in X$ and $y \in Y$, weight the edges of the subgraph $G \square e$ by $w$ so that each vertex $\left(u_{G}, x\right) \in V(G \square H)$ has colour $c\left(u_{G}, t_{1}\right)$ and $\left(u_{G}, y\right) \in V(G \square H)$ has colour $c\left(u_{G}, t_{2}\right)$. Assign every other edge of $G \square H$ weight $a$. Call this weighting $\phi$ and the induced colouring $c_{\phi}$. 
If $u_{H} \in X$, then $c_{\phi}(u)=c\left(u_{G}, t_{1}\right)+(d-1) a$, and if $u_{H} \in Y$, then $c_{\phi}(u)=c\left(u_{G}, t_{2}\right)+(d-1) a$. Two vertices are adjacent if either their $H$-coordinates agree and they are adjacent in a copy of $G$ or if their $G$-coordinates agree and they are adjacent in a copy of $H$. In the former case, their colours are distinct under $\phi$ since they are distinct under $w$. In the latter, consider two adjacent vertices $u=\left(u_{G}, u_{H}\right)$ and $u^{\prime}=\left(u_{G}, u_{H}^{\prime}\right)$ where $u_{H} \in X, u_{H}^{\prime} \in Y$. By choice of $w, c\left(u_{G}, t_{1}\right) \neq c\left(u_{G}, t_{2}\right)$ and so $\phi(u) \neq \phi\left(u^{\prime}\right)$. Thus $\phi$ is an $\{a, b\}$-weight colouring of $G \square H$.

Corollary 3.4.5. If $G$ and $H$ are regular bipartite graphs, then the following graphs are 2-weight colourable:

1. $K_{n} \square H$, if $n \geq 4$;

2. $C_{n} \square H$ if $n \geq 4, n \neq 5$;

3. $G \square H$.

Proof. Applying Theorem 3.4.4 to Propositions 3.4.2 and 3.4.3 immediately gives results 1 and 2, respectively. For 3 , since $K_{2} \square K_{2} \cong C_{4}, K_{2} \square K_{2}$ is 2-weight colourable by Proposition 3.2.9. By Theorem 3.4.4, $K_{2} \square H$ is 2-weight colourable; applying Theorem 3.4.4 again implies that $G \square H$ is 2-weight colourable.

On a related note, Davoodi and Omooni [37] claim to have recently proven that, for any two bipartite graphs $G$ and $H$, one has that $\chi_{\Sigma}^{e}(G \square H) \leq 2$ if $G \square H \neq K_{2}$. Their manuscript also states that, for any two graphs $G$ and $H$, $\chi_{\Sigma}^{e}(G \square H) \leq \max \left\{\chi_{\Sigma}^{e}(G), \chi_{\Sigma}^{e}(H)\right\}$ (though not stated in their paper, one should assume that both $G$ and $H$ are nice, or adopt the convention that $\left.\chi_{\Sigma}^{e}\left(K_{2}\right):=\infty\right)$. 


\subsection{Graphs which are not 2-weight colourable}

By using a class of "gadget" graphs, one can explicitly construct infinite classes of graphs which may not be $\{a, b\}$-weight coloured for infinitely many pairs $\{a, b\}$. These gadgets are themselves 2-weight colourable, but they have the property that in any of their 2-weight colourings, certain edges receive a predetermined weight.

The graph $\widehat{K}_{n}$ is defined to be the graph obtained from $K_{n}$ by subdividing one edge exactly once.

Proposition 3.5.1. For $n \geq 4$, the graph $\widehat{K}_{n}$ is 2 -weight colourable. Moreover, in any 2-weight colouring of $\widehat{K}_{n}$, the edges incident to its degree 2 vertex must receive the same colour.

Proof. Let $x$ be the vertex of $\widehat{K}_{n}$ of degree 2 and let $u, v$ be its neighbours. An $\{a, b\}$ weight colouring of $\widehat{K}_{4}$ is given on the left hand side in Figure 8 on Page 83 , where the dotted edges have non-zero weight; assume $n \geq 5$.

Let $K_{n}$ be obtained by adding the edge $u v$ to $\widehat{K}_{n}-x$. By Lemma 3.2 .5 , there exists an edge weighting $w$ of $K_{n}$ such that all the vertices have distinct colours except for $u$ and $v$. Moreover, $c(u)=c(v)=r a+(n-1-r) b$, where $r \in\{\lfloor n / 2\rfloor,\lceil n / 2\rceil-1\}$. Assign the weight $w(u v)$ from $K_{n}$ to the edges $x u$ and $x v$ in $\widehat{K}_{n}$. Note that $w$ is an $\{a, b\}$-weight colouring as long as $c(u)=c(v) \neq c(x)$. Clearly $c(x) \in\{2 a, 2 b\}$. Since $a$ and $b$ are set arbitrarily in Lemma 3.2.5, one may assume that $c(x)=2 a$. If $c(u) \neq 2 a$, then the weighting properly colours $V(G)$. Suppose that $c(u)=2 a$.

- If $n$ is odd, then the edge weighting $w^{\prime}$ given by swapping every edge's weight gives $c^{\prime}(u)=c(u)=2 a \neq 2 b=c^{\prime}(x)$.

- If $n$ is even, then, by the construction of the weighting in Lemma 3.2.5, 
$c(u)=\frac{n}{2} a+\left(\frac{n}{2}-1\right) b$. So, $2 a=\frac{n}{2}(a+b)-b$. If the edge weighting $w^{\prime}$ given by swapping every edge's weight gives a conflict between $u$ and $x$, then $2 b=\frac{n}{2}(a+b)-a$. Together, these imply that $a=b$, a contradiction.

Thus $\widehat{K}_{n}$ admits an $\{a, b\}$-weight colouring.

To prove the second part of the proposition, suppose $\widehat{K}_{n}$ is the smallest counterexample for which there exists an $\{a, b\}$-weight colouring $w$ such that $w(x u) \neq w(x v)$. It can be easily seen that $\widehat{K}_{4}$ does not admit such edge weighting; assume $n \geq 5$. Note that there exists no vertex $y \in V\left(\widehat{K}_{n}\right) \backslash\{u, v\}$ such that $c(y) \in\{(n-1) a,(n-1) b\}$, otherwise $\widehat{K}_{n}-y$ would be a smaller counterexample. Therefore, since $w$ induces a proper vertex colouring and all the colours (except for that of $x$ ) are of the form $r a+(n-1-r) b$ for some $0 \leq r \leq n-1$, it must be that $c(u), c(v) \in\{(n-1) a,(n-1) b\}$. By removing $u, v$, and $x$, an $\{a, b\}$-weight colouring of $K_{n-2}$ is obtained, a contradiction to Corollary 3.2.4.

Corollary 3.5.2. Let $G$ be a graph, $x \in V(G)$, and let $G^{\prime}$ be obtained by identifying a $x$ with the degree 2 vertex of $\widehat{K}_{n}$. If $G^{\prime}$ is 2 -weight colourable, then the edges in $\widehat{K_{n}}$ incident to $x$ must receive the same colour.

Proof. Since the proof of Proposition 3.5.1 did not depend in any way on the accumulated colour at vertex $x$, then regardless of graph joined to $\widehat{K_{n}}$ at $x$, the two edges incident with $x$ in $\widehat{K_{n}}$ must still receive the same weight.

An example is given on the left of Figure 8 , found on page 83 , where $G=K_{2}$ and $n=4$. The weight of the leaf's edge is forced to be equal to that of its adjacent edges; this is another useful gadget. It is shown on the right of Figure 8. In both cases the dotted edges represent one class of weights in the forced colouring; on the 
left, the dotted edges must receive non-zero weight, while on the right the solid edges must receive non-zero weight.
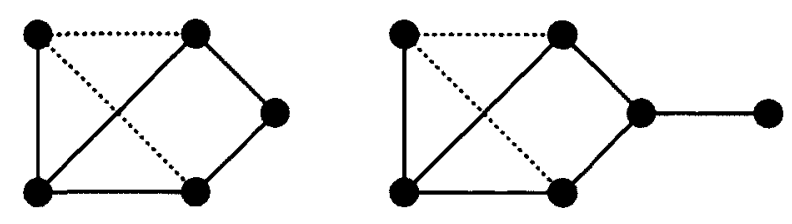

Figure 8: 2-weight colourable graphs with forced weightings

By applying Proposition 3.5.1, which established the weight colourability of $\widehat{K_{n}}$, the following examples of graphs which are not 2-weight colourable may be constructed.

1. Join two copies of $\widehat{K_{4}}$ by an edge attached at their vertices of degree 2 .

2. Join $2 n+1$ copies of $\widehat{K_{4}}$ to a $C_{2 n+1}$ by an edge attaching the degree 2 vertex in each copy of $\widehat{K}_{4}$ to a distinct cycle vertex.

To see why the graph defined in 2 , which we denote $H$, cannot be $S$-weight coloured for any set $S$ of size 2, consider the accumulated colour at one of the cycle vertices, say $v$. Since $H$ is a 3 -regular graph, $c(v) \in\{3 a, 2 a+b, a+2 b, 3 b\}$. If $c(v)=3 a$, then the edge incident to $v$ which is not in the cycle $C_{2 n+1}$, say $e$, must have weight $a$ and, as shown in Figure 8, so must the two edges in the copy of $\widehat{K_{4}}$ joined to $v$ by an edge. Thus both endpoints of $e$ would have colour $3 a$. A similar argument shows that $c(v) \neq 3 b$. Thus the only possible accumulated colours on cycle vertices are $2 a+b$ and $2 b+a$. Since an odd cycle cannot be properly 2 -coloured, $H$ cannot be 2 -weight coloured. 
Proposition 3.5.3. Let $0 \leq a \in \mathbb{Z}$ and $d \mid a$. Let $H$ be a graph and $G$ be a graph obtained from identifying a vertex $u$ of $H$ with a vertex of a $K_{n}$ (all other vertices of $H$ and $K_{n}$ being disjoint). If

$$
\operatorname{deg}_{H}(u)<\left(\frac{d}{a+d}\right)\left\lfloor\frac{n-1}{2}\right\rfloor
$$

then $G$ is not $\{a, a+d\}$-weight colourable. Furthermore, if

$$
\operatorname{deg}_{H}(u)=\left(\frac{d}{a+d}\right)\left\lfloor\frac{n-1}{2}\right\rfloor,
$$

then in any $\{a, a+d\}$-weight colouring of $G$, all edges in $H$ incident to $u$ must receive weight $a+d$.

Proof. Suppose $d=1$, and suppose that $w$ is an $\{a, a+1\}$-weight colouring of $G$. Every vertex of $K_{n}-u$ has colour $r a+(n-1-r)(a+1)=(n-1)(a+1)-r$ for some $0 \leq r \leq n-1$ and both of the colours $(n-1) a$ and $(n-1)(a+1)$ cannot appear simultaneously on $K_{n}-u$. If $c(u)<(n-1)(a+1)$, then there are only $n-1$ colours available for the vertices of $K_{n}$, a contradiction. So $c(u) \geq(n-1)(a+1)$.

Let $\left.w\right|_{K_{n}}$ be the restriction of $w$ to $K_{n}$. By Corollary $3.2 .4, K_{n}$ is not $\{a, a+1\}$ weight colourable. Thus, there must be exactly two vertices of $K_{n}$ with the same colour given by $\left.w\right|_{K_{n}}$ and $u$ must be one such vertex. By Lemma 3.2.5, $\left.w\right|_{K_{n}}(u)=r a+(n-1-r)(a+1)=(n-1)(a+1)-r$, where $r \in\{\lfloor n / 2\rfloor,\lceil n / 2\rceil-1\}$. Note that $r \geq\lfloor(n-1) / 2\rfloor$. If $u$ is incident with $s$ edges of weight $a$ in $H$, then

$$
(n-1)(a+1) \leq c(u)=r a+(n-1-r)(a+1)+s a+\left(\operatorname{deg}_{H}(u)-s\right)(a+1),
$$


which simplifies to $\operatorname{deg}_{H}(u) \geq\left(\frac{1}{a+1}\right)(r+s)$. Hence $\operatorname{deg}_{H}(u) \geq\left(\frac{1}{a+1}\right)\left(\left\lfloor\frac{n-1}{2}\right\rfloor+s\right)$, contradicting the fact that $\operatorname{deg}_{H}(u)<\left(\frac{1}{a+1}\right)\left\lfloor\frac{n-1}{2}\right\rfloor$. Also, if $\operatorname{deg}_{H}(u)=\left(\frac{1}{a+1}\right)\left\lfloor\frac{n-1}{2}\right\rfloor$ then $s=0$, proving the second claim.

Now, let $d>1$ be any positive divisor of $a$. By Proposition 3.2.1, $G$ has an $\{a, a+d\}$-weight colouring if and only if it has a $\left\{\frac{a}{d}, \frac{a}{d}+1\right\}$-weight colouring. If $\operatorname{deg}_{H}(u)<\left(\frac{1}{a / d+1}\right)\left\lfloor\frac{n-1}{2}\right\rfloor$, then $G$ has no $\left\{\frac{a}{d}, \frac{a}{d}+1\right\}$-weight colouring by the above argument. Hence, if $\operatorname{deg}_{H}(u)<\left(\frac{d}{a+d}\right)\left\lfloor\frac{n-1}{2}\right\rfloor$, then $G$ has no $\{a, a+d\}$-weight colouring. The second result follows similarly.

Proposition 3.5.3 may be used to construct many graphs which are not $\{a, a+1\}$ weight colourable and so, in particular, are not $\{1,2\}$-weight colourable. In fact, if $H$ is any graph and $u$ any vertex of $H$, then there exists a sufficiently large $n$ such that attaching $K_{n}$ to $u$ (and only to $u$ ) gives a graph which is not $\{a, a+1\}$ weight colourable. The equality condition may also be used to construct graphs with no $\{a, a+1\}$-weight colouring (for a specific $a$ ). For example, the graph obtained by joining two copies of $K_{n}, n \geq 5$, with a path of length 3 , say $e_{1}, e_{2}, e_{3}$, is not $\{a, a+1\}$-weight colourable for $a=\left\lfloor\frac{n-1}{2}\right\rfloor-1$ since the weights of $e_{1}$ and $e_{3}$ are forced to be $a+1$, and thus the ends of $e_{2}$ will receive the same colour.

In general, we do not know whether or not there exists a graph which is uniquely $\{1,2,3\}$-weight colourable, up to isomorphism, nor graphs which require three edge weights to properly vertex colour the graph by sums where certain edges are forced to receive a particular weight. If such graphs were known to exist, it is possible that they could be used as gadgets to prove or disprove the 1-2-3 Conjecture. 


\section{Chapter 4}

\section{List Weightings Which Colour Graph}

\section{Elements by Sums}

As discussed in the introductory chapters, it is natural to generalize colouring and weighting problems to their list versions. By doing so, stronger results are obtained and, often, insight is gained into the original problem. Some natural (and powerful) tools which one typically uses to analyze graph functions, such as algebraic methods or the probabilistic method, lend themselves well to these list variations. This is indeed the case for the List 1-2-3 Conjecture and List 1-2 Conjecture.

List 1-2-3 Conjecture (Bartnicki, Grytczuk, Niwcyk [15]). For every nice graph G, $\operatorname{ch}_{\Sigma}^{e}(G) \leq 3$.

List 1-2 Conjecture (Przybyło, Wozniak [77]). For every graph $G, \operatorname{ch}_{\Sigma}^{t}(G) \leq 2$.

The results in this section are largely inspired by the work of Bartnicki, Grytczuk, and Niwczyk [15], who present an approach to the List 1-2-3 Conjecture (and, thus, the 1-2-3 Conjecture) which focuses on the analysis of a natural colouring polynomial. The most natural way to analyze this polynomial is via Alon's algebraic tool known 
as the Combinatorial Nullstellensatz.

To date, all that is known about $\operatorname{ch}_{\Sigma}^{e}(G)$ and $\operatorname{ch}_{\Sigma}^{t}(G)$ are bounds for a handful of graphs, noted in Section 2.4. This chapter represents significant progress on the study of vertex colourings from list-weightings. In Section 4.1, we will show how the Combinatorial Nullstellensatz is used to study these list-weightings. The major results in Section 4.2 are the first known bounds on $\operatorname{ch}_{\Sigma}^{e}(G)$ and $\operatorname{ch}_{\Pi}^{e}(G)$, and the first non-trivial bound on $\operatorname{ch}_{\Sigma}^{t}(G)$. Better bounds are subsequently obtained for graph products in Section 4.3. Finally, in Section 4.4, the list variation of additive colourings is considered (that is, vertex list-weightings which properly colour vertices by sums); the approach in this section is constructive rather than algebraic.

\subsection{Polynomials, permanents, and the Combina- torial Nullstellensatz}

Let $G=(V, E)$ be a graph, with $E(G)=\left\{e_{1}, \ldots, e_{m}\right\}$ and $V(G)=\left\{v_{1}, \ldots, v_{n}\right\}$. Associate with each $e_{i}$ the variable $x_{i}$ and with each $v_{j}$ the variable $x_{m+j}$. Define two more variables for each $v_{j} \in V(G): X_{v_{j}}=\sum_{e_{i} \ni v_{j}} x_{i}$ and $Y_{v_{j}}=x_{m+j}+X_{v_{j}}$. For an an orientation $D$ of $G$, define the following two polynomials, where $l=m+n$ :

$$
\begin{aligned}
P_{D}\left(x_{1}, \ldots, x_{m}\right) & =\prod_{(u, v) \in E(D)}\left(X_{v}-X_{u}\right) \\
T_{D}\left(x_{1}, \ldots, x_{l}\right) & =\prod_{(u, v) \in E(D)}\left(Y_{v}-Y_{u}\right) .
\end{aligned}
$$

Let $w$ be an edge weighting of $G$. By letting $x_{i}=w\left(e_{i}\right)$ for $1 \leq i \leq m, w$ is a proper vertex colouring by sums if and only if $P_{D}\left(w\left(e_{1}\right), \ldots, w\left(e_{m}\right)\right) \neq 0$. A similar 
conclusion can be made about $T_{D}$ if $w$ is a total weighting of $G$.

This leads us to the problem of determining when the polynomials $P_{D}$ and $T_{D}$ do not vanish everywhere, i.e., when there exist values of the variables for which the polynomial is non-zero. Alon's famed Combinatorial Nullstellensatz gives sufficient conditions to guarantee that a polynomial does not vanish everywhere; as stated in the introduction to the chapter, this theorem is the primary tool used to prove the results of Sections 4.2 and 4.3 .

Combinatorial Nullstellensatz (Alon [8]). Let $\mathbb{F}$ be an arbitrary field, and let $f=f\left(x_{1}, \ldots, x_{n}\right)$ be a polynomial in $\mathbb{F}\left[x_{1}, \ldots, x_{n}\right]$. Suppose the total degree of $f$ is $\sum_{i=1}^{n} t_{i}$, where each $t_{i}$ is a nonnegative integer, and suppose the coefficient of $\prod_{i=1}^{n} x_{i}^{t_{i}}$ in $f$ is nonzero. If $S_{1}, \ldots, S_{n}$ are subsets of $\mathrm{F}$ with $\left|S_{i}\right|>t_{i}$, then there are $s_{1} \in S_{1}, s_{2} \in S_{2}, \ldots, s_{n} \in S_{n}$ so that

$$
f\left(s_{1}, \ldots, s_{n}\right) \neq 0
$$

For a polynomial $P \in \mathbb{F}\left[x_{1}, \ldots, x_{l}\right]$ and a monomial term $M$ of $P$, let $h(M)$ be the largest exponent of any variable in $M$. The monomial index of $P$, denoted mind $(P)$, is the minimum $h(M)$ taken over all monomials of $P$. Define the graph parameters $\operatorname{mind}(G):=\operatorname{mind}\left(P_{D}\right)$ and $\operatorname{tmind}(G):=\operatorname{mind}\left(T_{D}\right)$, where $D$ is an orientation of $G$. Note that, given a graph $G$ and two orientations $D$ and $D^{\prime}$, $P_{D}\left(x_{1}, \ldots, x_{l}\right)= \pm P_{D^{\prime}}\left(x_{1}, \ldots, x_{l}\right)$; a similar argument holds for $T_{D}$. The parameters $\operatorname{mind}(G)$ and $\operatorname{tmind}(G)$ are hence well-defined. Note that, for any graph $G$, $\operatorname{tmind}(G) \leq \operatorname{mind}(G)$

The following lemma is obtained by applying the Combinatorial Nullstellensatz 
to $P_{D}$ and $T_{D}$ :

Lemma 4.1.1. Let $G$ be a graph and $k$ a positive integer.

1. (Bartnicki, Grytczuk, Niwczyk [15]) If $G$ is nice and $\operatorname{mind}(G) \leq k$, then $\operatorname{ch}_{\Sigma}^{e}(G) \leq k+1$.

2. (Przybyło, Woźniak [77]) If $\operatorname{tmind}(G) \leq k$, then $\operatorname{ch}_{\Sigma}^{t}(G) \leq k+1$.

The following proposition will also be useful.

Proposition 4.1.2. If $G$ is a graph with connected components $G_{1}, G_{2}, \ldots, G_{k}$, then $\operatorname{mind}(G)=\max \left\{\operatorname{mind}\left(G_{i}\right): 1 \leq i \leq k\right\}$

Proof. For each $i=i, \ldots, k$, let $D_{i}$ be an orientation of $G_{i}$ and $D$ be the resulting orientation of $G$. Since $P_{D}=P_{D_{1}} P_{D_{2}} \cdots P_{D_{k}}$ and, for any $1 \leq i<j \leq k$, the variables of $P_{D_{i}}$ are distinct from those of $P_{D_{j}}$, the highest exponent in any mononial term of $P_{D}$ is equal to the highest exponent of any monomial term among $P_{D_{1}}, \ldots, P_{D_{k}}$. The result immediately follows.

In [15], Bartnicki et al. show how one may study the permanent of particular $\{-1,0,1\}$-matrices in order to gain insight on $\operatorname{mind}(G)$ and $\operatorname{tmind}(G)$. Let $\mathbb{M}(m, n)$ denote the set of all real valued matrices with $m$ rows and $n$ columns, and $\mathbb{M}(m)$ denote the set of square $m \times m$ matrices. The permanent of a matrix $A \in \mathbb{M}(m)$, denoted per $A$, is calculated as follows:

$$
\operatorname{per} A=\sum_{\sigma \in S_{m}} \prod_{i=1}^{m} a_{i, \sigma(i)}
$$

Note that the formulas for the permanent and determinant of a matrix are very similar, with the formula for per $A$ lacking the alternating signs used in the calculation 
of $\operatorname{det} A$. Unfortunately, this difference is substantial in terms of the complexity of the calculations. Ryser's formula, given below, is one of the fastest known algorithms for permanent computation, yet it requires $O\left(2^{n} n^{2}\right)$ arithmetic operations to compute the permanent of an $n \times n$ matrix:

Ryser's Formula (Ryser [78]). If $A$ is an $n \times n$ square matrix, then

$$
\operatorname{per}(A)=(-1)^{n} \sum_{S \subseteq[n]}(-1)^{|S|} \prod_{i=1}^{n} \sum_{j \in S} a_{i j}
$$

Furthermore, Valiant [86] showed that the problem of determining per $A$ is \#P-complete, and hence belongs to a class of problem believed to be more difficult to compute than NP (where many NP problems ask "Are there any solutions...?", a \#P problem asks "How many...?").

Fortunately, some properties of determinants hold for permanents. For instance, a permanent may be calculated using expansion along a row or column. Consider the following $3 \times 3$ matrix $A$ :

$$
A=\left(\begin{array}{ccc}
1 & -1 & 2 \\
3 & 0 & 5 \\
-1 & 4 & 6
\end{array}\right)
$$

Expansion along the first row gives the determinant

$$
\begin{aligned}
\operatorname{det} A & =(1)[(0)(6)-(5)(4)]-(-1)[(3)(6)-(5)(-1)]+(2)[(3)(4)-(0)(-1)] \\
& =27
\end{aligned}
$$


whereas the same expansion for the permanent gives

$$
\begin{aligned}
\operatorname{per} A & =(1)[(0)(6)+(5)(4)]+(-1)[(3)(6)+(5)(-1)]+(2)[(3)(4)+(0)(-1)] \\
& =31
\end{aligned}
$$

The permanent may also be defined for a general matrix $A \in \mathbb{M}(m, n)$ if $n \geq m$. Let $Q_{m, n}$ denote the set of sequences of length $m$ with entries from $[n]$ which contain no repetition of elements; such sequences are also known as $m$-permutations from $[n]$. For example, $Q_{2,3}=\{(1,2),(1,3),(2,1),(2,3),(3,1),(3,2)\}$. The permanent of $A$ is defined as follows:

$$
\operatorname{per} A=\sum_{\alpha \in Q_{m, n}} \prod_{i=1}^{m} a_{i, \alpha(i)}
$$

For example, if

$$
B=\left(\begin{array}{ccc}
1 & -1 & 2 \\
3 & 0 & 5
\end{array}\right)
$$

then

$$
\text { per } B=(1)(0)+(1)(5)+(-1)(3)+(-1)(5)+(2)(3)+(2)(0)=3
$$

Note that the terms may be regrouped according to the $2 \times 2$ submatrices of $B$ :

$$
\begin{aligned}
\operatorname{per} B & =[(1)(0)+(-1)(3)]+[(1)(5)+(2)(3)]+[(-1)(5)+(2)(0)] \\
& =\operatorname{per}\left(\begin{array}{cc}
1 & -1 \\
3 & 0
\end{array}\right)+\text { per }\left(\begin{array}{ll}
1 & 2 \\
3 & 5
\end{array}\right)+\operatorname{per}\left(\begin{array}{cc}
-1 & 2 \\
0 & 5
\end{array}\right)
\end{aligned}
$$

The following proposition generalizes this regrouping observation: 
Proposition 4.1.3. Let $A \in \mathbb{M}(m, n), n \geq m$. Let $\left\{B_{i} \mid 1 \leq i \leq\left(\begin{array}{c}n \\ m\end{array}\right)\right\}$ be the set of all $m \times m$ submatrices of $A$. The permanent of $A$ is

$$
\operatorname{per} A=\sum_{i=1}^{\left(\begin{array}{c}
n \\
m
\end{array}\right)} \operatorname{per} B_{i}
$$

The permanent rank of a matrix $A$ (not necessarily square) is the size of the largest square submatrix of $A$ having nonzero permanent. Let $A^{(k)}=[A A \cdots A]$ denote the matrix formed of $k$ consecutive copies of $A$. If $A$ has size $m \times l$, then the permanent index of $A$ is the smallest $k$, if it exists, such that $A^{(k)}$ has permanent rank $m$. This parameter is denoted pind $(A)$. If such a $k$ does not exist, then $\operatorname{pind}(A):=\infty$. Alternately, $\operatorname{pind}(A)$ is the smallest $k$ such that a square matrix of size $m$ having nonzero permanent can be constructed by taking columns from $A$, each column taken no more than $k$ times.

There are three matrices related to directed graphs which will be of interest:

Definition 4.1.4. Let $G=(V, E)$ be a graph, $V(G)=\left\{v_{1}, \ldots, v_{n}\right\}$, $E(G)=\left\{e_{1}, \ldots, e_{m}\right\}$. For an orientation $D$ of $G$, define the matrices $A_{D} \in \mathbb{M}(m)$, $B_{D} \in \mathbb{M}(m, n)$, and $M_{D} \in \mathbb{M}(m, m+n)$ as follows:

$$
\text { - } A_{D}=\left(a_{i, j}\right) \text { where } a_{i, j}=\left\{\begin{array}{cl}
1 & \text { if } e_{j} \text { is incident with the head of } e_{i} \\
-1 & \text { if } e_{j} \text { is incident with the tail of } e_{i} \\
0 & \text { otherwise }
\end{array}\right.
$$


- $B_{D}=\left(b_{i, j}\right)$ where $b_{i, j}=\left\{\begin{array}{cl}1 & \text { if } v_{j} \text { is the head of } e_{i} \\ -1 & \text { if } v_{j} \text { is the tail of } e_{i} \\ 0 & \text { otherwise }\end{array}\right.$

- $M_{D}=\left(A_{D} \mid B_{D}\right)$.

For example, consider the digraph $D$ in Figure 9 and its associated matrices given in Figure 10.

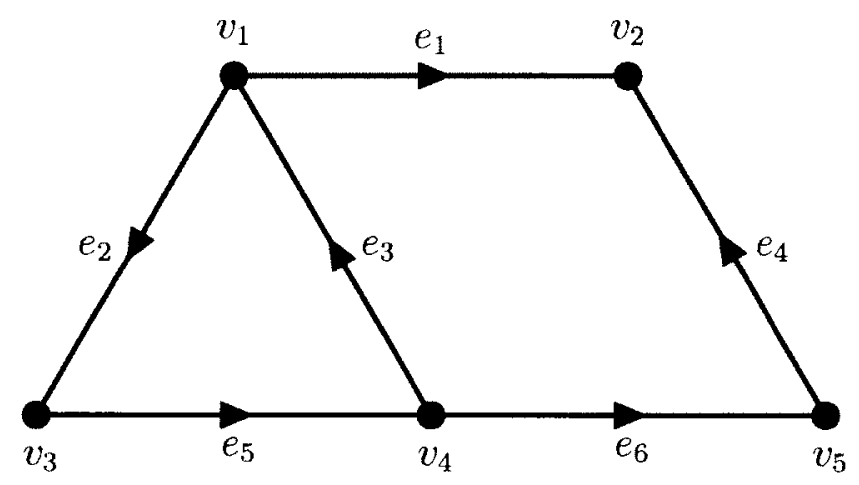

Figure 9: A digraph used to illustrate $A_{D}, B_{D}$, and $M_{D}$

$M_{D}=\left(A_{D} \mid B_{D}\right)=\left(\begin{array}{cccccc|ccccc}0 & -1 & -1 & 1 & 0 & 0 & -1 & 1 & 0 & 0 & 0 \\ -1 & 0 & -1 & 0 & 1 & 0 & -1 & 0 & 1 & 0 & 0 \\ 1 & 1 & 0 & 0 & -1 & -1 & 1 & 0 & 0 & -1 & 0 \\ 1 & 0 & 0 & 0 & 0 & -1 & 0 & 1 & 0 & 0 & -1 \\ 0 & -1 & 1 & 0 & 0 & 1 & 0 & 0 & -1 & 1 & 0 \\ 0 & 0 & -1 & 1 & -1 & 0 & 0 & 0 & 0 & -1 & 1\end{array}\right)$

Figure 10: The associated matrices of $D$ from Figure 9 
The following lemmas, which relate the matrices $A_{D}, B_{D}$, and $M_{D}$ to the polynomials $P_{D}$ and $T_{D}$, provide the fundamental link between the graphic polynomials of interest and matrix permanents:

Lemma 4.1.5 (Bartnicki, Grytczuk, Niwczyk [15]). Let $A=\left(a_{i j}\right) \in \mathbb{M}(m)$ have finite permanent index. If $P\left(x_{1}, \ldots, x_{m}\right)=\prod_{i=1}^{m}\left(a_{i 1} x_{1}+\ldots+a_{i m} x_{m}\right)$, then $\operatorname{mind}(P)=\operatorname{pind}\left(A_{D}\right)$

The proof is omitted, but the result follows from the fact that the coefficient of $x_{1}^{k_{1}} x_{2}^{k_{2}} \cdots x_{m}^{k_{m}}$ in the expansion of $P$ is equal to $\frac{\operatorname{per}(M)}{k_{1} ! \cdots k_{m} !}$ where $M$ is the $m \times m$ matrix where column $a_{j}$ from $A$ appears $k_{j}$ times. Lemma 4.1 .5 immediately implies the following vital link between the (total) monomial index of a graph $G$ and the permanent index of $A_{D}$ (respectively, $T_{D}$ ) for any orientation $D$ of $G$ :

Lemma 4.1.6. Let $D$ be an orientation of a graph $G$.

1. (Bartnicki, Grytczuk, Niwczyk [15]) If $G$ is nice, then $\operatorname{mind}(G)=\operatorname{pind}\left(A_{D}\right)$.

2. (Przybyło, Woźniak [77]) For any graph $G, \operatorname{tmind}(G)=\operatorname{pind}\left(M_{D}\right)$.

Lemmas 4.1.1 and 4.1.6 imply the following relationship between $\mathrm{ch}_{\Sigma}^{e}(G)$ and $A_{D}$, and $\operatorname{ch}_{\Sigma}^{t}(G)$ and $T_{D}$ :

Corollary 4.1.7. Let $G$ be a graph, $D$ an orientation of $G$, and $k$ a positive integer.

1. If $G$ is nice and $\operatorname{pind}\left(A_{D}\right) \leq k$, then $\operatorname{ch}_{\Sigma}^{e}(G) \leq k+1$.

2. If $\operatorname{pind}\left(M_{D}\right) \leq k$, then $\operatorname{ch}_{\Sigma}^{t}(G) \leq k+1$.

Consider the following illustrative example of what we henceforth call "the permanent method." Let $D$ be the digraph in Figure 9 on page 93 and let $G$ be its 
underlying simple graph. The associated polynomial $P_{D}$ is:

$$
\begin{aligned}
P_{D}\left(x_{1}, \ldots, x_{6}\right) & =\left(x_{1}+x_{4}-x_{1}-x_{2}-x_{3}\right) \times\left(x_{2}+x_{5}-x_{1}-x_{2}-x_{3}\right) \\
& \times\left(x_{1}+x_{2}+x_{3}-x_{3}-x_{5}-x_{6}\right) \times\left(x_{1}+x_{4}-x_{4}-x_{6}\right) \\
& \times\left(x_{3}+x_{5}+x_{6}-x_{2}-x_{5}\right) \times\left(x_{4}+x_{6}-x_{3}-x_{5}-x_{6}\right) \\
& =\left(x_{4}-x_{2}-x_{3}\right) \times\left(x_{5}-x_{1}-x_{3}\right) \times\left(x_{1}+x_{2}-x_{5}-x_{6}\right) \\
& \times\left(x_{1}-x_{6}\right) \times\left(x_{3}+x_{6}-x_{2}\right) \times\left(x_{4}-x_{3}-x_{5}\right)
\end{aligned}
$$

Recalling $M_{D}$, note that the coefficients of each factor in $P_{D}$ correspond to the entries in each row of $A_{D}$ :

$$
M_{D}=\left[A_{D} \mid B_{D}\right]=\left(\begin{array}{cccccc|ccccc}
0 & -1 & -1 & 1 & 0 & 0 & -1 & 1 & 0 & 0 & 0 \\
-1 & 0 & -1 & 0 & 1 & 0 & -1 & 0 & 1 & 0 & 0 \\
1 & 1 & 0 & 0 & -1 & -1 & 1 & 0 & 0 & -1 & 0 \\
1 & 0 & 0 & 0 & 0 & -1 & 0 & 1 & 0 & 0 & -1 \\
0 & -1 & 1 & 0 & 0 & 1 & 0 & 0 & -1 & 1 & 0 \\
0 & 0 & -1 & 1 & -1 & 0 & 0 & 0 & 0 & -1 & 1
\end{array}\right) .
$$

Since per $A_{D}=-4 \neq 0$, we have $\operatorname{pind}\left(A_{D}\right)=1$ (each column from $A_{D}$ is chosen once). The coefficient of the monomial $x_{1} x_{2} x_{3} x_{4} x_{5} x_{6}$ in $P_{D}$ also nonzero by Lemma 4.1.5. This gives $\operatorname{mind}(G)=1$ (the highest exponent in the term $x_{1} x_{2} x_{3} x_{4} x_{5} x_{6}$ is 1 ), and hence $\operatorname{ch}_{\Sigma}^{e}(G) \leq 2$ by the Combinatorial Nullstellensatz. Since there are adjacent vertices of equal degree in $G, \chi_{\Sigma}^{e}(G) \neq 1$, implying $\operatorname{ch}_{\Sigma}^{e}(G) \neq 1$ and so $\operatorname{ch}_{\Sigma}^{e}(G)=2$. 


\subsection{Bounds for edge list-weightings and total list- weightings}

Armed with the Combinatorial Nullstellensatz and the permanent method, we may now proceed with the major results of this chapter. The following theorem summarizes two major results which will be proven on vertex colouring list-weightings:

Theorem 4.2.1. If $G$ is a nice graph with maximum degree $\Delta(G)$, then $\operatorname{ch}_{\Sigma}^{e}(G) \leq 2 \Delta(G)+1$. If $G$ is any graph, then $\operatorname{ch}_{\Sigma}^{t}(G) \leq\left\lceil\frac{2}{3} \Delta(G)\right\rceil+1$.

As mentioned, the first result gives the first known upper bound on $\operatorname{ch}_{\Sigma}^{e}(G)$; the second is an improvement on the trivial bound on $\operatorname{ch}_{\Sigma}^{t}(G)$ of $\Delta(G)+1$ (stated in Proposition 2.4.6).

Theorem 4.2 .1 is proven by establishing bounds on $\operatorname{mind}(G)$ and $\operatorname{tmind}(G)$ using the permanent method. One important tool is the following lemma, a generalization of a similar result in [15]:

Lemma 4.2.2 (Przybyło, Woźniak [77]). Let $A$ be an $m \times l$ matrix, and let $L$ be an $m \times m$ matrix where each column of $L$ is a linear combination of columns of $A$. Let $n_{j}$ denote the number of columns of $L$ in which the $j^{\text {th }}$ column of $A$ appears with nonzero coefficient. If $\operatorname{per} L \neq 0$, then $\operatorname{pind}(A) \leq \max \left\{n_{j} \mid j=1, \ldots l\right\}$.

We will also find the following theorem useful, which gives a method for constructing graphs in a way that preserves the property of having low monomial index:

Theorem 4.2.3 (Bartnicki, Grytczuk, Niwczyk [15]). Let $G$ be a simple graph with $\operatorname{mind}(G) \leq 2$. Let $U$ be a nonempty subset of $V(G)$. If $F$ is a graph obtained by 
adding two new vertices $u, v$ to $V(G)$ and joining them to each vertex of $U$, and $H$ is a graph obtained from $F$ by joining $u$ and $v$, then $\operatorname{mind}(F), \operatorname{mind}(H) \leq 2$.

As a consequence, the following graph classes have low monomial index and hence small values of $\operatorname{ch}_{\Sigma}^{e}(G)$ :

Corollary 4.2.4 (Bartnicki, Grytczuk, Niwczyk [15]). If $G$ is a complete graph, a complete bipartite graph, or tree, then $\operatorname{mind}(G) \leq 2$ and hence $\operatorname{ch}_{\Sigma}^{e}(G) \leq 3$.

It can also be easily shown that the same bound holds for cycles.

Proposition 4.2.5. If $G=C_{n}$, then $\operatorname{mind}(G) \leq 2$ and hence $\operatorname{ch}_{\Sigma}^{e}(G) \leq 3$.

Proof. Let $V(G)=\left\{v_{1}, v_{2}, \ldots, v_{n}\right\}$ and $E(G)=\left\{v_{1} v_{2}, v_{2} v_{3}, \ldots, v_{n-1} v_{n}, v_{n} v_{1}\right\}$. Let $D$ be the orientation of $G$ with $A(D)=\left\{\left(v_{1}, v_{2}\right),\left(v_{2}, v_{3}\right), \ldots,\left(v_{n-1}, v_{n}\right),\left(v_{n}, v_{1}\right)\right\}$. Consider the colouring polynomial

$$
P_{D}=\left(x_{2}-x_{n}\right)\left(x_{3}-x_{1}\right)\left(x_{4}-x_{2}\right) \cdots\left(x_{n}-x_{n-2}\right)\left(x_{1}-x_{n-1}\right) .
$$

Since each variable appears in exactly two factors of $P_{D}$, no exponent in the expansion of $P_{D}$ exceeds 2 , and hence $\operatorname{mind}(G) \leq 2$.

In order to prove Theorem 4.2.1, the following generalization of Theorem 4.2 .3 is required:

Lemma 4.2.6. Let $G$ be a graph with finite monomial index $\operatorname{mind}(G) \geq 1$. Let $U$ be a nonempty subset of $V(G)$. If $F$ is a graph obtained by adding two new vertices $u, v$ to $V(G)$ and joining them to each vertex of $U$, and $H$ is a graph obtained from $F$ by joining $u$ and $v$, then $\operatorname{mind}(F), \operatorname{mind}(H) \leq \max \{2, \operatorname{mind}(G)\}$. 
The proof which follows is an adaptation of the proof of Theorem 4.2.3 in [15]. Given a matrix $A$ with columns $a_{1}, a_{2}, \ldots, a_{n}$ and a sequence of (not necessarily distinct) column indices $K=\left(i_{1}, i_{2}, \ldots, i_{k}\right), A(K)$ is defined to be the matrix $A(K)=\left(\begin{array}{llll}a_{i_{1}} & a_{i_{2}} & \cdots & a_{i_{k}}\end{array}\right)$.

Proof. Let $U=\left\{u_{1}, \ldots, u_{k}\right\}$ be the subset of $V(G)$ stated in the theorem. Let $E_{u}=\left\{e_{1}, e_{3}, \ldots, e_{2 k-1}\right\}$ and $E_{v}=\left\{e_{2}, e_{4}, \ldots, e_{2 k}\right\}$ be the sets of edges incident to the vertices $u$ and $v$, respectively. Assume that these edges are oriented toward $U$, and that for each $i=1,2, \ldots, k$ the edges $e_{2 i-1}$ and $e_{2 i}$ have the same head.

Let $D$ be an orientation of $F, D^{\prime}$ the induced orientation of $G$, and consider the matrices $A_{D}$ and $A_{D^{\prime}}$. Let $A_{1}, \ldots, A_{2 k}$ be the first $2 k$ columns of $A_{D}$, corresponding to $\left\{e_{1}, e_{2}, \ldots, e_{2 k}\right\}$. If we write $A=\left(A_{1} \cdots A_{2 k}\right)$, then $A_{F}=(A B)$ where $B=\left(\begin{array}{c}X \\ A_{D^{\prime}}\end{array}\right)$.

Let $Y$ be the $(2 k) \times(2 k)$ matrix and $Z$ the $(|E(F)|-2 k) \times(2 k)$ matrix such that $A=\left[\begin{array}{l}Y \\ Z\end{array}\right]$. Since the edges $e_{2 i-1}$ and $e_{2 i}$ have the same head for each $i=1,2, \ldots, k$, $A_{2 i-1}$ and $A_{2 i}$ agree on $Z$. Furthermore, $Y$ may be written as a block matrix, where $\left(\begin{array}{ll}0 & 1 \\ 1 & 0\end{array}\right)$ occupies the diagonals and $\left(\begin{array}{cc}-1 & 0 \\ 0 & -1\end{array}\right)$ is everywhere else, as seen in Figure 11.

$$
Y=\left(\begin{array}{ccccccc}
0 & 1 & -1 & 0 & & -1 & 0 \\
1 & 0 & 0 & -1 & \cdots & 0 & -1 \\
-1 & 0 & 0 & 1 & & -1 & 0 \\
0 & -1 & 1 & 0 & & 0 & -1 \\
& \vdots & & & \ddots & & \\
-1 & 0 & -1 & 0 & & 0 & 1 \\
0 & -1 & 0 & -1 & & 1 & 0
\end{array}\right)
$$

Figure 11: The block matrix $Y$ 
There exists a matrix of columns from $A_{D^{\prime}}$, with no column used more than $\operatorname{mind}(G)$ times, with nonzero permanent. Let $K$ denote the sequence of edges of $G$ which index this matrix. Consider a new matrix

$$
M=\left(\begin{array}{lllllll}
M_{1} & M_{1} & M_{2} & M_{2} & \cdots & M_{k} & M_{k} B(K)
\end{array}\right)
$$

where $M_{j}=A_{2 j-1}-A_{2 j}$ for $j=1,2, \ldots, k$.

The properties of the columns of $A$ outlined above imply that the matrix $M$ can be written as follows: $M=\left(\begin{array}{cc}R & X(K) \\ 0 & A_{D^{\prime}}(K)\end{array}\right)$, where $R$ has all constant rows:

$$
R=\left(\begin{array}{ccccc}
-1 & -1 & -1 & & -1 \\
1 & 1 & 1 & \cdots & 1 \\
-1 & -1 & -1 & & -1 \\
& \vdots & & \ddots & \\
1 & 1 & 1 & & 1
\end{array}\right) .
$$

Since per $M=\operatorname{per} R \times \operatorname{per} A_{D^{\prime}}(K)$, each of per $R$ and $\operatorname{per} A_{G}(K)$ are nonzero, and any column of $M$ appears in the linear combination of at most 2 columns of $R$, Lemma 4.2.2 implies that $\operatorname{mind}(F) \leq \max \{2, \operatorname{mind}(G)\}$.

Now consider the matrix $A_{H}$. Let $e_{0}=u v$, and orient it from $v$ to $u$. The matrix $A_{H}$ is precisely $A_{F}$ with a row and column added for $e_{0}$ (say, as the first row and column). It can be depicted in block form $A_{H}=\left(\begin{array}{cc}Y^{\prime} & X^{\prime} \\ Z^{\prime} & A_{G}\end{array}\right)$, where $Y^{\prime}$ and $Z^{\prime}$ are the matrices depicted in Figure 12 on page 100.

Let $A_{0}, A_{1}, \ldots, A_{2 k}$ denote the first $2 k+1$ columns of $A_{H}$, corresponding to the 


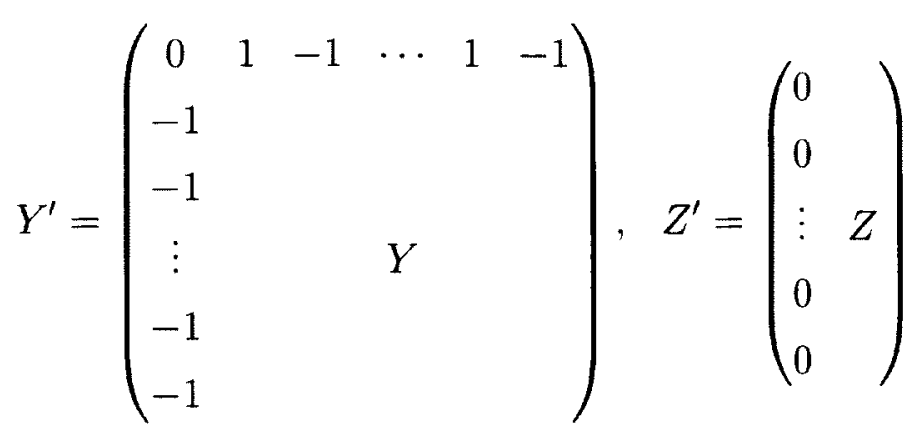

Figure 12: The matrices $Y^{\prime}$ and $Z^{\prime}$

edges $e_{0}, e_{1}, \ldots, e_{2 k}$. Form a new matrix

$$
N=\left(\begin{array}{lllllllll}
N_{0} & N_{0} & N_{1} & N_{2} & N_{2} & \cdots & N_{k} & N_{k} & B(K)
\end{array}\right)
$$

so that $N_{0}=A_{0}$ and $N_{j}=A_{2 j-1}-A_{2 j}$ for $j=1,2, \ldots, k$. Arguing as before, $N=\left(\begin{array}{cc}R^{\prime} & X^{\prime}(K) \\ 0 & A_{G}(K)\end{array}\right)$, where $R^{\prime}$ is the following square matrix:

$$
R^{\prime}=\left(\begin{array}{cccccc}
0 & 0 & 2 & 2 & & 2 \\
-1 & -1 & -1 & -1 & \cdots & -1 \\
-1 & -1 & 1 & 1 & & 1 \\
& \vdots & \vdots & & \ddots & \\
-1 & -1 & 1 & 1 & & 1
\end{array}\right)
$$

It is shown in [15] that per $R^{\prime} \neq 0$. Hence per $N=\operatorname{per} R^{\prime} \times \operatorname{per} A_{G}(K) \neq 0$, and since any column of $N$ appears in the linear combination of at most 2 columns of $R^{\prime}$, Lemma 4.2 .2 implies thats $\operatorname{mind}(H) \leq \max \{2, \operatorname{mind}(G)\}$. 
We may now proceed with the proof of Theorem 4.2.1. By carefully orienting the edges of a graph, applying the lemmas above will give us a matrix with non-zero permanent; applying the permanent method gives the result.

Theorem 4.2.7. If $G$ is a nice graph on at least 3 vertices, then $\operatorname{mind}(G) \leq 2 \Delta(G)$.

Proof. We first show that the statement is true for connected graphs on 3 or 4 vertices. If $G$ is a tree, cycle, or complete graph, then $\operatorname{mind}(G) \leq 2$ by Corollary 4.2 .4 and Proposition 4.2.5, and hence the theorem holds for the following graphs: $P_{3}, K_{3}, P_{4}$, $K_{1,3}, C_{4}$, and $K_{4}$. There are two remaining graphs on 4 vertices to consider: $K_{3}$ with a leaf, and $C_{4}$ with a chord. Consider the orientation of $K_{3}$ with a leaf, given in Figure 13. The associated polynomial is $P_{D}=\left(x_{2}+x_{4}\right)\left(x_{3}-x_{1}-x_{4}\right)\left(x_{4}-x_{2}\right)\left(x_{1}+x_{2}-x_{3}\right)$; the coefficient of $x_{1}^{2} x_{2}^{2}$ is nonzero, and hence $\operatorname{mind}(G) \leq 3<6=2 \Delta(G)$.

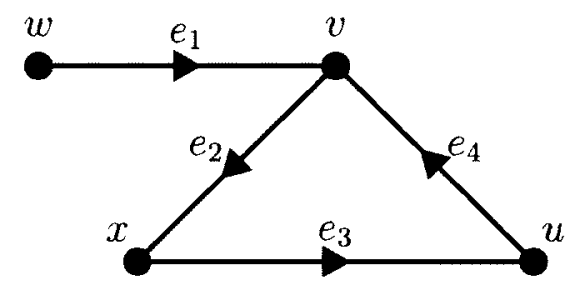

Figure 13: An orientation of $K_{3}$ with a leaf

Suppose now that $G$ is $C_{4}$ with a chord, oriented as in Figure 14 and with vertices and edge as given in Figure 14 (see page 102). The associated polynomial is

$$
P_{D}=\left(x_{2}-x_{4}-x_{5}\right)\left(-x_{1}+x_{3}\right)\left(-x_{2}+x_{4}+x_{5}\right)\left(x_{1}-x_{3}-x_{5}\right)\left(-x_{1}-x_{2}+x_{3}+x_{4}\right),
$$

and the coefficient of $x_{1} x_{2} x_{3} x_{4}^{2}$ is nonzero. Hence mind $(G) \leq 3<6=2 \Delta(G)$. 


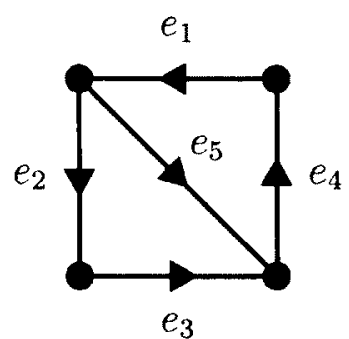

Figure 14: An orientation of $C_{4}$ with a chord

We proceed now by induction on $|V(G)|$. We may assume that $G$ is connected, since Proposition 4.1.2 states that $\operatorname{mind}(G)$ is at most the largest monomial index of its components. Let $G$ be a connected graph on at least 5 vertices, and for any graph $H$ with $|V(H)|<|V(G)|$, assume that $\operatorname{mind}(H) \leq 2 \Delta(H)$.

We may assume that $G$ is not a complete graph, and hence there exist $u, v, w \in V(G)$ such that the induced subgraph $G[\{u, v, w\}]$ is a path of length 2 (or, $u v w$ is an induced 2-path). Choose this 2-path such that $d(u)+d(w)$ is minimum. The ultimate goal will be to apply an inductive argument to $G-\{u, w\}$, however we must concern ourselves with whether or not this subgraph of $G$ is nice. To this end, we define the following sets of edges:

$$
\begin{aligned}
\mathcal{F} & =\text { the edges of those components in } G-\{u, w\} \text { isomorphic to } K_{2} \\
E_{u} & =\{e \in E(G) \mid e \ni u, e \neq u v\} \\
E_{w} & =\{e \in E(G) \mid e \ni w, e \neq v w\} \\
E_{v} & =\{e \in E(G) \mid e \ni v, e \neq u v, v w\} \\
E^{*} & =E(G) \backslash\left(E_{u} \cup E_{v} \cup E_{w} \cup\{u v, v w\}\right)
\end{aligned}
$$


The path $u v w$ and the sets of edges $E_{u}, E_{v}, E_{w}$ are shown in Figure 15.

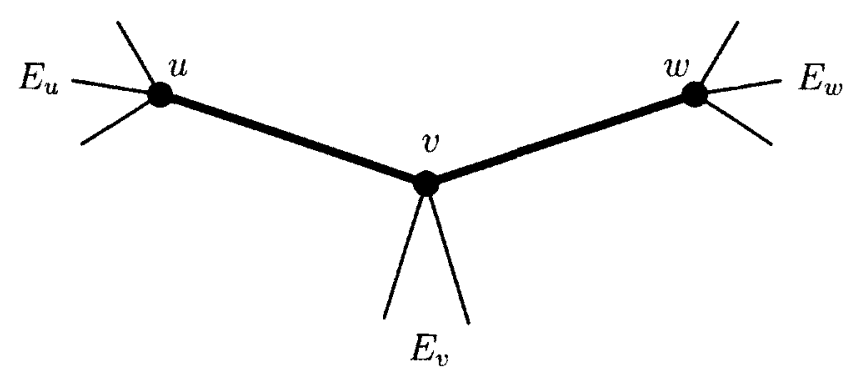

Figure 15: The induced 2-path $u v w$ in $G$

Case 1: $E_{v} \cap \mathcal{F} \neq \emptyset$

We first consider the case that deleting $u$ and $w$ leaves a component isomorphic to $K_{2}$ containing the vertex $v$. Suppose $E_{v} \cap \mathcal{F} \neq \emptyset$. There can be only one edge in this intersection, otherwise the connected component containing $v$ in $G-\{u, w\}$ would have two or more edges. This implies that, since $u v, v w \in E(G)$, we have that $N_{G}(v)=\{u, w, x\}$ for some vertex $x \in V(G)$. Since $\{v, x\}$ induces a graph isomorphic to $K_{2}$ in $G-\{u, w\}$, we have that $N_{G}(x) \subseteq\{u, v, w\}$.

If $x$ is adjacent to both $u$ and $w$, then $v$ and $x$ are adjacent twins. Suppose that $G \backslash\{v, x\}$ is not nice; we will show that this contradicts the choice of $u v w$ which minimizes $d_{G}(u)=d_{G}(w)$. If $G$ is not nice, then $u, w$, or both $u$ and $w$ are adjacent to exactly one vertex in $G$ other than $v$ and $x$; without loss of generality, suppose that $u y \in E(G), y \neq v, x$. Since $y \notin N_{G}(x)$, the vertices $y, u, x$ induce a 2-path; furthermore, $d_{G}(y)+d_{G}(x)=1+3=4$. This contracts our choice of $u v w$, since $d(u)+d(w) \geq 3+2=5$. Thus, $G-\{v, x\}$ is a nice graph, and so, by Lemma $4.2 .6, \operatorname{mind}(G) \leq \max \{2, \operatorname{mind}(G-\{v, x\})\}$. By the induction hypothesis, 
$\operatorname{mind}(G-\{v, x\}) \leq 2 \Delta(G-\{v, x\})$, and so

$$
\operatorname{mind}(G) \leq \max \{2, \operatorname{mind}(G-\{v, x\})\} \leq \max \{2,2 \Delta(G-\{v, x\})\} \leq 2 \Delta(G)
$$

We may now assume that $x$ is not adjacent to at least one of $u$ and $w$. If $w \notin N_{G}(x)$, then both $u v w$ and $x v w$ are induced 2-paths in $G$. By the minimality of $d(u)+d(w)$, we must have that $d(u) \leq d(v)$. If $u$ is adjacent to $x$, then $d(v)=2$ and, since $u$ is adjacent to $v$ as well, $d(u)=2$ and $N_{G}(u)=\{v, x\}$. Otherwise, if $u$ is not adjacent to $x$, then $d_{G}(u)=d_{G}(x)=1$ and $N_{G}(u)=\{v\}$. In either case, $u$ and $x$ are twins. If $G-\{u, x\}$ is not nice, then the only edge not incident to $u$ or $x$ is the edge $v w$, contradicting our choice of $G$ with $|V(G)| \geq 5$. Assume that $G-\{u, x\}$ is nice. By Lemma 4.2.6, $\operatorname{mind}(G) \leq \max \{2, \operatorname{mind}(G-\{u, x\})\}$, and by the induction hypothesis, $\operatorname{mind}(G-\{u, x\}) \leq 2 \Delta(G-\{u, x\}))$. Thus,

$$
\operatorname{mind}(G) \leq \max \{2, \operatorname{mind}(G-\{u, x\})\} \leq \max \{2,2 \Delta(G-\{u, x\})\} \leq 2 \Delta(G)
$$

If $u \notin N_{G}(x)$ and $w \in N_{G}(x)$, then the exact same argument holds as for $u \in N_{G}(x)$ and $w \notin N_{G}(x)$. Having considered all possible neighbourhoods of $x$, we conclude that if $E_{v} \cap \mathcal{F}$ is nonempty, then $\operatorname{mind}(G) \leq 2 \Delta(G)$.

Case 2: $E_{v} \cap \mathcal{F}=\emptyset$

Suppose that $E_{v} \cap \mathcal{F}=\emptyset$. The argument proceeds as follows: after choosing a "good" orientation $D$ of $G$, we will construct a matrix whose columns are linear combinations of $A_{D}$ with no column of $A_{D}$ being used more than $2 \Delta(G)$ times and with nonzero permanent. The result will then follow by Lemma 4.2.2. 
Let $D$ be an orientation of $G$ where the edges of $E_{u} \cup\{u v\}$ and $E_{v}$ are oriented toward $u$ and $v$, respectively, and the edges of $E_{w} \cup\{v w\}$ are oriented away from $w$; see Figure 16. Let $c_{u v}$ and $c_{v w}$ be the columns of $A_{D}$ associated with the edges $u v$

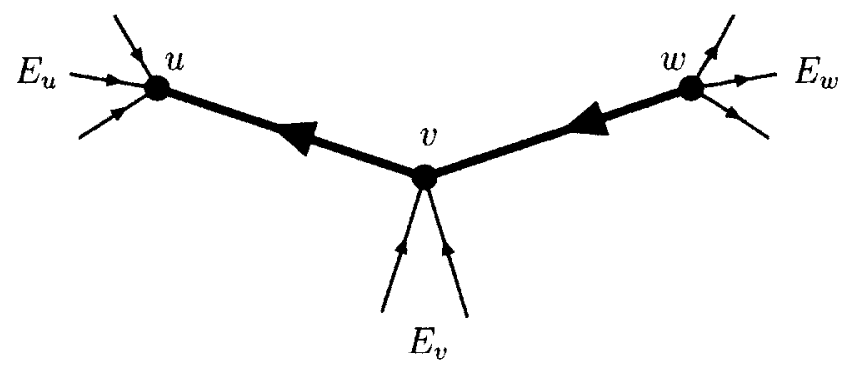

Figure 16: An orientation $D$ of a graph $G$ with an induced 2-path uvw

and $v w$, respectively, and let $c=c_{u v}-c_{v w}$; see Figure 17 .

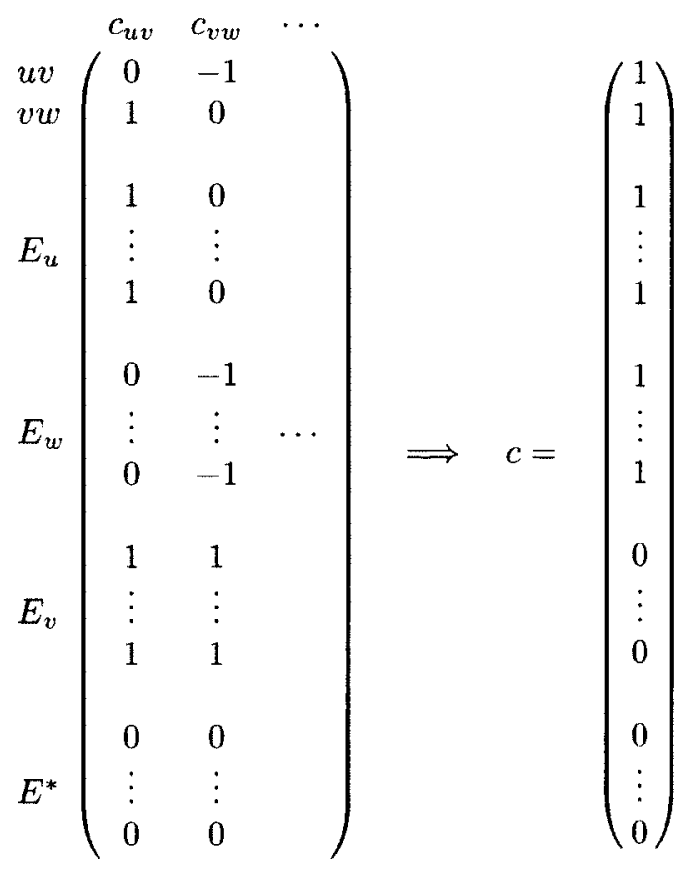

Figure 17: An operation on two columns of $A_{D}$ 
We must still concern ourselves with the possibility that deleting $u$ and $w$ from $G$ gives a graph which is not nice. If a component of $G-\{u, w\}$ is isomorphic to $K_{2}$, then one vertex of this component must be adjacent to either $u$ or $w$ in $G$. Let $\mathcal{F}=\left\{f_{1}, \ldots, f_{k}\right\}$. For each $f_{i} \in \mathcal{F}$, let $e_{i}$ be an edge from $E_{u}$ or $E_{w}$ to which $f_{i}$ is adjacent. Let $F$ denote this collection of edges from $E_{u} \cup E_{w}$, and let $F_{u}=\left\{e: e \in E_{u} \cap F\right\}$ and $F_{w}=\left\{e: e \in E_{w} \cap F\right\}$.

Let $H=G-\{u, w\}-\mathcal{F}$ and $D(H)$ be the corresponding sub-digraph of $D$. Since we have removed all components isomorphic to $K_{2}, H$ is nice. Since $H$ has fewer vertices than $G$, by the induction hypothesis, $\operatorname{mind}(H) \leq 2 \Delta(H)$. Hence, there exists a matrix $L_{H}$ consisting of columns of $A_{D(H)}$, none repeated more than $2 \Delta(H)$ times, with $\operatorname{per}\left(L_{H}\right) \neq 0$. Let $K$ denote the sequence of edges which indexes the columns of $L_{H}$. Recall that, for an $m \times n$ matrix $A, A^{(k)}$ is the $m \times k n$ matrix consisting of $k$ consecutive copies of $A$ (see page 92). Let $L_{G}$ be the following block matrix:

$$
L_{G}=\left(c^{(d(u)+d(w))}\left|A_{D}(F)\right| A_{D}(K)\right)=\begin{array}{r}
E_{u} \cup E_{w} \cup\{u v, v w\} \\
\mathcal{F}(H)
\end{array}\left(\begin{array}{ccc}
J_{d(u)+d(w)} & K_{1} & X_{1} \\
0 & K_{2} & X_{2} \\
0 & 0 & L_{H}
\end{array}\right) \text {, }
$$

where the blocks are as follows:

- $J_{d(u)+d(w)}$ is the $(d(u)+d(w)) \times(d(u)+d(w))$ all 1's matrix.

- $K=\left(\begin{array}{l}K_{1} \\ K_{2}\end{array}\right)$ having entries in the column corresponding to $e_{i} \in F_{u}$ as follows:

- 1 in each row indexed by the other edges from $E_{u}$,

- 1 in the row indexed by $u v$,

- -1 in the row indexed by $f_{i}$, and 
- 0 in all other entries.

If $e_{i} \in F_{w}$, the entries follow the same pattern with the signs swapped. Since the column associated with $e_{i}$ has only one non-zero entry in the rows indexed by $\mathcal{F}, K_{2}$ is diagonal with $\left|F_{u}\right|$ entries being -1 and $\left|F_{w}\right|$ entries being 1.

- $X=\left(\begin{array}{c}X_{1} \\ X_{2}\end{array}\right)$, the $(|E(G)|-|E(H)|) \times|E(H)|$ submatrix of $A_{D}(K)$ whose rows are indexed by $E(G) \backslash E(H)$; and

- $L_{H}$, is the matrix with per $\left(L_{H}\right) \neq 0$ defined above.

Since $J_{d(u)+d(w)}, K_{2}$, and $L_{H}$ are all square matrices,

$$
\begin{aligned}
\operatorname{per}\left(L_{G}\right) & =\operatorname{per}\left(J_{d(u)+d(w)}\right) \cdot \operatorname{per}\left(K_{2}\right) \cdot \operatorname{per}\left(L_{H}\right) \\
& =(d(u)+d(w)) ! \cdot(-1)^{\left|F_{u}\right|}(1)^{\left|F_{u}\right|} \cdot \operatorname{per}\left(L_{H}\right) \neq 0
\end{aligned}
$$

Since the sets $\{u v, v w\}, F$, and $E(H)$ are pairwise disjoint, no column is used more than $\max \{d(u)+d(w), 1, \operatorname{mind}(H)\}$ times. Lemma 4.2 .2 states that $\operatorname{pind}\left(A_{D}\right) \leq \max \{d(u)+d(w), 1, \operatorname{mind}(H)\}$. Since $\operatorname{pind}\left(A_{D}\right)=\operatorname{mind}(G)($ Lemma 4.1.6.1) and $\operatorname{mind}(H) \leq 2 \Delta(H)$ by induction,

$$
\operatorname{mind}(G) \leq \max \{d(u)+d(w), 1, \operatorname{mind}(H)\} \leq \max \{2 \Delta(G), 1,2 \Delta(H)\} \leq 2 \Delta(G)
$$

Theorem 4.2.8. If $G$ is any graph, then $\operatorname{tmind}(G) \leq\left\lceil\frac{2}{3} \Delta(G)\right\rceil$.

Proof. The proof follows as that of Theorem 4.2.7, with a different matrix of interest. Assume that, for any graph $H$ with $|V(H)|<|V(G)|, \operatorname{tmind}(H) \leq\left\lceil\frac{2}{3} \Delta(H)\right\rceil$. Choose an induced 2-path uvw as before, and assume that $d(u) \leq d(w)$. Let $L_{G}^{\prime}$ be 
the following matrix consisting of linear combinations of columns from $M_{D}$ for the orientation $D$ of $G$ :

$$
L_{G}^{\prime}=\left(c_{u}^{(r)}\left|c_{w}^{(s)}\right| c^{(t)}\left|A_{D}(F)\right| A_{D}(K)\right)
$$

where

$$
r=\min \left\{d(u),\left\lceil\frac{d(u)+d(w)}{3}\right\rceil\right\}, \quad s=\left\lceil\frac{d(u)+d(w)}{3}\right\rceil, \quad t=d(u)+d(w)-r-s
$$

Let $A^{*}$ be the following matrix:

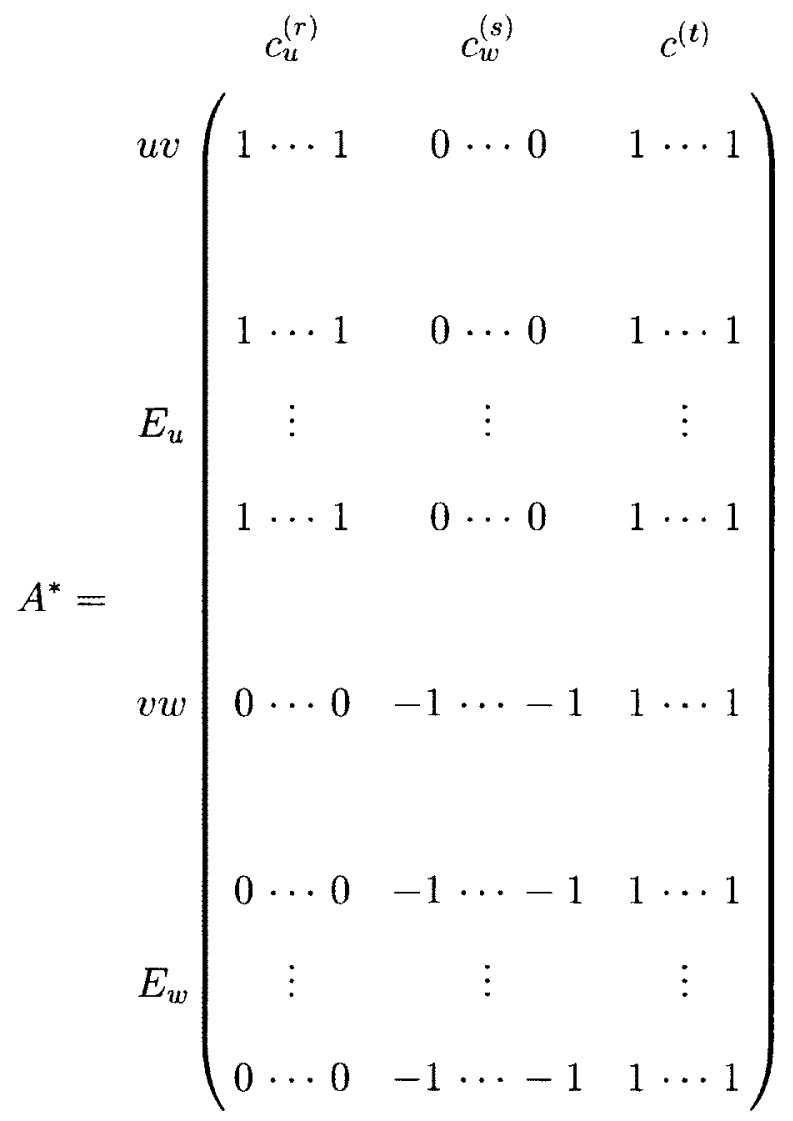


The matrix $L_{G}^{\prime}$ can then be rewritten as a block matrix:

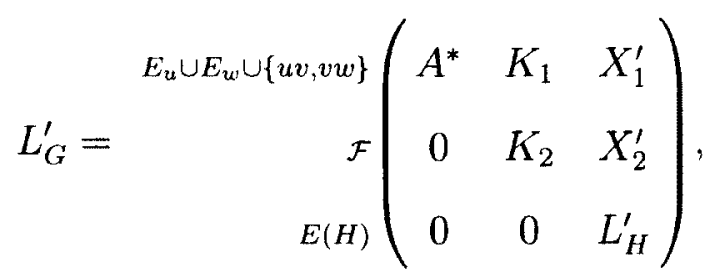

where $K_{1}$ and $K_{2}$ are defined as in the proof of Theorem 4.2.7 and $L_{H}^{\prime}$ is the matrix guaranteed by the induction hypothesis with non-zero permanent and no column repeated more than $\operatorname{pind}\left(M_{D(H)}\right)=\operatorname{tmind}(H) \leq\left\lceil\frac{2 \Delta(H)}{3}\right\rceil$ times (recall that Lemma 4.1.6.2 states that $\left.\operatorname{pind}\left(M_{D(H)}\right)=\operatorname{tmind}(H)\right)$.

The permanent of $A^{*}$ is $\operatorname{per}\left(A^{*}\right)=r !\left(\begin{array}{c}d(u) \\ r\end{array}\right) \times s !\left(\begin{array}{c}d(w) \\ s\end{array}\right)(-1)^{s} \times t ! \neq 0$. Since $A^{*}, K_{2}$, and $L_{H}^{\prime}$ are all square matrices,

$$
\begin{aligned}
\operatorname{per}\left(L_{G}^{\prime}\right) & =\operatorname{per}\left(A^{*}\right) \cdot \operatorname{per}\left(K_{2}\right) \cdot \operatorname{per}\left(L_{H}\right) \\
& =\operatorname{per}\left(A^{*}\right) \cdot(-1)^{\left|F_{u}\right|}(1)^{\left|F_{w}\right|} \cdot \operatorname{per}\left(L_{H}\right) \neq 0 .
\end{aligned}
$$

We now consider the number of times the columns, $c_{u}, c_{w}$, and $c$ appear in $A^{*}$. If $r=d(u)$, then $t=d(w)-\left\lceil\frac{d(u)+d(w)}{3}\right\rceil \leq d(w)-\frac{d(w)}{3} \leq\left\lceil\frac{2 d(w)}{3}\right\rceil$. If $r=\left\lceil\frac{d(u)+d(w)}{3}\right\rceil$, then $t=d(u)+d(w)-2\left\lceil\frac{d(u)+d(w)}{3}\right\rceil \leq \frac{d(u)+d(w)}{3} \leq\left\lceil\frac{2 d(w)}{3}\right\rceil$. In either case, $\max \{r, s, t\} \leq\left\lceil\frac{2 d(w)}{3}\right\rceil$. Since the sets $F$ and $E(H)$ are pairwise disjoint, no column of $M_{D}$ is used more than $\max \left\{\left\lceil\frac{2 d(w)}{3}\right\rceil, 1, \operatorname{tmind}(H)\right\}$ times. Lemma 4.2 .2 states that $\operatorname{pind}\left(M_{D}\right) \leq \max \left\{\left\lceil\frac{2 d(w)}{3}\right\rceil, 1, \operatorname{tmind}(H)\right\}$, and so

$$
\begin{aligned}
\operatorname{tmind}(G) & \leq \max \left\{\left\lceil\frac{2 d(w)}{3}\right\rceil, 1, \operatorname{tmind}(H)\right\} \leq \max \left\{\left\lceil\frac{2 \Delta(G)}{3}\right\rceil,\left\lceil\frac{2 \Delta(H)}{3}\right\rceil\right\} \\
& \leq\left\lceil\frac{2 \Delta(G)}{3}\right\rceil .
\end{aligned}
$$


The proof of Theorem 4.2.1 easily follows.

Theorem 4.2.1. If $G$ is a nice graph with maximum degree $\Delta(G)$, then $\operatorname{ch}_{\Sigma}^{e}(G) \leq 2 \Delta(G)+1$. If $G$ is any graph, then $\operatorname{ch}_{\Sigma}^{t}(G) \leq\left\lceil\frac{2}{3} \Delta(G)\right\rceil+1$.

Proof. Lemma 4.1.1 states that $\operatorname{ch}_{\Sigma}^{e}(G) \leq \operatorname{mind}(G)+1$ and $\operatorname{ch}_{\Sigma}^{t}(G) \leq \operatorname{tmind}(G)+1$; applying Lemma 4.1.1 to Theorems 4.2.7 and 4.2.8 gives the desired result.

By Proposition 2.4.8, we immediately obtain a bound on $\operatorname{ch}_{\Pi}^{e}(G)$.

Theorem 4.2.9. If $G$ is a nice graph with maximum degree $\Delta(G)$, then $\operatorname{ch}_{\Pi}^{e}(G) \leq 4 \Delta(G)+2$.

Proof. Proposition 2.4.8 states that $\operatorname{ch}_{\Pi}^{e}(G) \leq 2 \operatorname{ch}_{\Sigma}^{e}(G)$, and so $\operatorname{ch}_{\Pi}^{e}(G) \leq 2(2 \Delta(G)+1)$.

Proposition 2.4 .8 also relates $\operatorname{ch}_{\Pi}^{t}(G)$ to $\operatorname{ch}_{\Pi}^{e}(G)$, however this relation shows that $\operatorname{ch}_{\Pi}^{t}(G) \leq 2\left(\left\lceil\frac{2}{3} \Delta(G)\right\rceil+1\right)$ and we know from Proposition 2.4.3 that $\operatorname{ch}_{\Pi}^{t}(G) \leq \operatorname{ch}(G) \leq \Delta(G)+1$.

In the proof of Theorem 4.2.7, no column corresponding to a vertex of the graph is chosen. As such, Theorems 4.2.7 and 4.2.8 imply the following results on $(k, l)$-weight choosability:

Corollary 4.2.10. If $G$ is a nice graph, then it is $(1,2 \Delta(G)+1)$-weight choosable.

Corollary 4.2.11. If $G$ is any graph, then it is $\left(\left\lceil\frac{2}{3} \Delta(G)\right\rceil+1,\left\lceil\frac{2}{3} \Delta(G)\right\rceil+1\right)$-weight choosable.

By following the proof of Theorem 4.2.8 and letting $r=s=1$ and $t=d(u)+d(w)-2$, the following result is similarly obtained: 
Corollary 4.2.12. If $G$ is a nice graph, then it is $(2,2 \Delta(G)-1)$-weight choosable.

Recall that Przybyło's proof that $\chi_{\Sigma}^{t}(G) \leq 3[75]$ in fact shows that weights from $\{1,2\}$ for the vertices and $\{1,2,3\}$ for the edges of a graph admit a weighting which is a proper vertex colouring by sums. Corollary 4.2 .12 is thus particularly relevant, since a natural approach to the List 1-2 Conjecture is to ask for the smallest $k$ such that every graph is $(2, k)$-weight choosable.

\subsection{Colouring graph products by list-weightings}

Though the bounds presented in Section 4.2 represent progress on the List 1-2-3 and List 1-2 Conjectures, there is still room for improvement. As such, we will consider some classes of graphs where smaller upper bounds can be obtained.

The following lemma provides a decomposition approach for the classes of graphs which we will study:

Lemma 4.3.1. Let $G$ be a graph, and let $H$ be an induced subgraph of $G$ containing a 2-factor. Let $X$ be a minimal edge cut separating $V(H)$ from $V(G) \backslash V(H)$. If the components of $G-H-X$ are $C_{1}, \ldots, C_{k}$, then

$$
\begin{aligned}
\operatorname{mind}(G) & \leq \max \{|X|+\operatorname{mind}(H), \operatorname{mind}(G-X)\} \\
& =\max \left\{\operatorname{mind}(H)+|X|, \operatorname{mind}\left(C_{1}\right), \ldots, \operatorname{mind}\left(C_{k}\right)\right\}
\end{aligned}
$$

Proof. Let $|V(H)|=v$ and $F=\left\{e_{1}, \ldots, e_{v}\right\}$ be a 2 -factor of $H$. Let $D$ be an orientation of $G$ such that the cycles of $F$ are directed. Define the column vector $c=\sum_{i=1}^{v} c_{i}$ where $c_{i}$ is the column of $A_{D}$ corresponding to $e_{i}$. For each $e \in E(H) \backslash F$ 
there are two edges of $F$ incident to each of the head and tail of $e$, and for each $e \in F$ there is one edge of $F$ incident to each of the head and tail of $e$. Hence, the entries of $c$ are nonzero in the rows indexed by the edges of $X$ and 0 in all other entries.

There exists a matrix $L_{G-X}$ consisting of columns of $A_{G-X}$ with no column of $A_{D}$ repeated more than $\operatorname{mind}(G-X)$ times and $\operatorname{per}\left(L_{G-X}\right) \neq 0$. Let $K$ denote the sequence of edges of $G-X$ which index $A_{G-X}$. Consider the following matrix:

$$
L=\left(c^{(|X|)} A_{D}(K)\right)=\left(\begin{array}{cc}
M & N \\
0 & L_{G-X}
\end{array}\right)
$$

where $(M N)$ is indexed by $X$, each row of $M$ is constant, and every entry of $M$ is nonzcro. Any column indexed by $e \in E(G) \backslash F$ is used at most mind $(G-X)$ times in the construction of $L$, and any edge from $F$ is used at most $|X|+\operatorname{mind}(H)$ times. Clearly, $\operatorname{per}(L)=\operatorname{per}(M) \operatorname{per}\left(L_{G}-X\right) \neq 0$, and hence $\operatorname{mind}(G)=\max \{|X|+\operatorname{mind}(H), \operatorname{mind}(G-X)\}$. Since $\operatorname{mind}(G-X)=\max \left\{\operatorname{mind}\left(C_{1}\right), \ldots, \operatorname{mind}\left(C_{k}\right), \operatorname{mind}(H)\right\}$ by Proposition 4.1.2, the result follows.

Recall that the Cartesian product of two graphs $G$ and $H$, denoted by $G \square H$, is defined as the graph having vertex set $V(G) \times V(H)$ where two vertices $\left(u, u^{\prime}\right)$ and $\left(v, v^{\prime}\right)$ are adjacent if and only if either $u=v$ and $u^{\prime}$ is adjacent to $v^{\prime}$ in $H$ or $u^{\prime}=v^{\prime}$ and $u$ is adjacent to $v$ in $G$. Some results on Cartesian products of graphs were presented in Section 3.4; for instance, if $G$ and $H$ are regular and bipartite, then $\chi_{\Sigma}^{e}\left(K_{n} \square H\right), \chi_{\Sigma}^{e}\left(C_{t} \square H\right)$, and $\chi_{\Sigma}^{e}(G \square H)$ are at most 2 for $n \geq 4$, and $t \geq 4, t \neq 5$. Lemma 4.3.1 may be used to bound $\operatorname{ch}_{\Sigma}^{e}(G \square H)$ for many more graphs $G$ and $H$. 
Let $G$ be a graph, $T$ a tree, and let $\mathcal{V}=\left\{V_{t} \subseteq V(G): t \in V(T)\right\}$ be a family of subsets of $V(G)$ which are indexed by the vertices of $T$. The pair $(T, \mathcal{V})$ is called a tree-decomposition of $G$ if the follow three conditions are satisfied:

1. $V(G)=\bigcap_{t \in T} V_{t}$

2. for every $e \in E(G)$, there exists a $t \in V(T)$ such that both ends of $e$ are in $V_{t}$; and

3. if $v \in V(G)$ lies in two distinct $V_{s}, V_{t} \in \mathcal{V}$, then $v \in V_{r}$ for every $r \in V(T)$ which lies on the unique path connecting $s$ and $t$ in $T$.

The subsets of $V(G)$ in $\mathcal{V}$ are called the bags of the tree-decomposition $(T, \mathcal{V})$. The width of a tree-decomposition is $\max \left\{\left|V_{t}\right|-1: t \in V(T)\right\}$, and the tree-width of a graph $G$, denoted $\operatorname{tw}(G)$, is the minimum width of a tree-composition of $G$.

For proofs of the following well known propositions, see [38].

Proposition 4.3.2. If $H$ is a subgraph of $G$, then $\operatorname{tw}(H) \leq \operatorname{tw}(G)$.

A graph is chordal if it has no induced cycles of length 4 or more; every chordal graph has a vertex which is simplicial, that is a vertex whose neighbourhood induces a clique. A chordal completion of a graph $G$ is a chordal graph $H$ with vertex set $V(H)=V(G)$, containing $G$ as a subgraph, and with a minimal number of edges with respect to these properties. Note that, since $G$ is a subgraph of $K_{|V(G)|}$ which is chordal, $G$ has a chordal completion.

Proposition 4.3.3. Let $G$ be a graph. If $H$ is a chordal completion of $G$, then $\operatorname{tw}(G)=\omega(H)-1$.

The following consequence of Proposition 4.3.3 will be particularly useful: 
Corollary 4.3.4. Every graph $G$ has a vertex $v \in V(G)$ such that $d_{G}(v) \leq \operatorname{tw}(G)$.

Proof. Let $H$ be a chordal completion of $G$. If $v$ is a simplicial vertex of $H$, then $d_{G}(v) \leq d_{H}(v) \leq \omega(H)-1=\operatorname{tw}(G)$.

We now prove the following result on Cartesian products of graphs. Note that, for the graph $G \square H$ and vertex $v \in V(G)$, the subgraph induced by the set of vertices $\{(v, x): x \in V(H)\}$ is denoted $(v, H)$.

Theorem 4.3.5. Let $H$ be a regular graph on $n \geq 3$ vertices which contains a 2-factor. If $G$ is a graph with tree-width $\operatorname{tw}(G)=t$, then $(1) \operatorname{mind}(G \square H) \leq n t+\operatorname{mind}(H)$ and $(2) \operatorname{ch}_{\Sigma}^{e}(G \square H) \leq n t+\operatorname{mind}(H)+1$.

Proof. We may assume that $G$ is connected. The proof of (1) is by induction on $|V(G)| ;$ the statement is true when $G$ is a single vertex, since $\operatorname{tw}(G)=0$ and $\operatorname{ch}_{\Sigma}^{e}(H) \leq \operatorname{mind}(H)+1$ is guaranteed by Lemma 4.1.1.

Suppose $|V(G)| \geq 2$. By Corollary 4.3.4, there is a $v \in V(G)$ such that $d_{G}(v) \leq \operatorname{tw}(G)$. Let $X$ be the minimal edge cut for $(v, H)$. Since $|X|=n \cdot d_{G}(v)$ and $\operatorname{tw}(G-v) \leq \operatorname{tw}(G)$ by Proposition 4.3.2, Lemma 4.3.1 implies that

$$
\begin{aligned}
\operatorname{mind}(G \square H) & \leq \max \left\{\operatorname{mind}(H)+n d_{G}(v), \operatorname{mind}((G \square H)-X)\right\} \\
& \leq \max \{\operatorname{mind}(H)+n t, \operatorname{mind}((G \square H)-(v, H)\} \\
& \leq \max \{\operatorname{mind}(H)+n t, \operatorname{mind}((G-v) \square H)\} \\
& \leq \max \{\operatorname{mind}(H)+n t, n \operatorname{tw}(G-v)+\operatorname{mind}(H)\} \\
& \leq \operatorname{mind}(H)+n t .
\end{aligned}
$$

Part (2) follows directly from Lemma 4.1.1. 
Since $\operatorname{mind}\left(K_{n}\right)$ and $\operatorname{mind}\left(C_{n}\right)$ are at most 2 (Corollary 4.2.4 and Proposition 4.2.5), the following corollary is obtained:

Corollary 4.3.6. For any integer $n \geq 3$ and any graph $G$

1. $\operatorname{ch}_{\Sigma}^{e}\left(G \square K_{n}\right) \leq n \cdot \operatorname{tw}(G)+3$, and

2. $\operatorname{ch}_{\Sigma}^{e}\left(G \square C_{n}\right) \leq n \cdot \operatorname{tw}(G)+3$.

In particular, if $G=T$ is a tree, then $\operatorname{ch}_{\Sigma}^{e}\left(T \square K_{n}\right) \leq n+1$ and $\operatorname{ch}_{\Sigma}^{e}\left(T \square C_{n}\right) \leq n+1$. Since $\Delta\left(T \square K_{n}\right)$ and $\Delta\left(T \square C_{n}\right)$ may be arbitrarily high for any fixed $n$, this gives a noticeable improvement on the bound from Theorem 4.2.1. More generally, if $G$ is $K_{4}$-minor free (equivalently, $G$ is series-parallel), then $\operatorname{tw}(G)=2$ and $\operatorname{ch}_{\Sigma}^{e}\left(G \square K_{n}\right) \leq 2 n+1$ and $\operatorname{ch}_{\Sigma}^{e}\left(G \square C_{n}\right) \leq 2 n+1$.

\subsection{Additive colourings from list-weightings}

In Section 2.3.2, we considered vertex weightings which properly colour the vertices of a graph by sums (among other operations). Czerwiński et al. [36] call such a weighting an additive colouring, and define the additive colouring number of $G$, denoted here by $\chi_{\Sigma}^{v}(G)$, to be the smallest $k$ for which $G$ has an additive colouring into $[k]$. They propose the following upper bound on $\chi_{\Sigma}^{v}(G)$ :

Additive Colouring Conjecture (Czerwiński, Grytczuk, Żelazny, [36]). For any graph $G, \chi_{\Sigma}^{v}(G) \leq \chi(G)$.

We will consider the usual list variation of this problem. Denote by $\operatorname{ch}_{\Sigma}^{v}(G)$ the smallest $k$ such that $G$ has an additive colouring from any assignment of lists of size $k$ 
to the vertices of $G$, and call $\operatorname{ch}_{\Sigma}^{v}(G)$ the additive choosability number of $G$. The following conjecture is offered in the spirit of the List 1-2-3 and List 1-2 Conjectures:

Additive List Colouring Conjecture. For any graph $G, \operatorname{ch}_{\Sigma}^{v}(G)=\chi_{\Sigma}^{v}(G)$.

One may directly try to show that $\operatorname{ch}_{\Sigma}^{v}(G) \leq \chi(G)$, which would also prove the Additive Colouring Conjecture (since clearly $\operatorname{ch}_{\Sigma}^{v}(G) \geq \chi_{\Sigma}^{v}(G)$ ).

First consider a simple class of graphs - complete graphs.

Proposition 4.4.1. If $G=K_{n}$ for any $n \geq 2$, then $\operatorname{ch}_{\Sigma}^{v}(G)=n$.

Proof. It is well known that $\operatorname{ch}(G)=n$; we will show additive list colourings of $K_{n}$ are precisely proper list-vertex colourings of $K_{n}$.

Assign to each vertex a list of real numbers. Let $\phi$ be a vertex colouring of $G$ from these lists, and let $c(v)=\sum_{u \in N_{G}(v)} \phi(u)$. Clearly $c(v)=\sum_{u \in N_{G}(v)} \phi(u)-\phi(v)$, and hence $c(u) \neq c(v) \Longleftrightarrow \phi(u) \neq \phi(v)$ for $u, v \in V(G)$.

Recall that the best published universal bound on $\chi_{\Sigma}^{v}(G)$, stated as Theorem 2.3.8, grows very large in terms of the acyclic chromatic number of $G$, whose best bounds, in turn, appear to grow very large with $\Delta(G)$. As a first step toward the Additive List Colouring Conjecture, the following theorem gives a much improved bound on $\chi_{\Sigma}^{v}(G)$ by showing that $\operatorname{ch}_{\Sigma}^{v}(G)$ is, at worst, quadratic in $\Delta(G)$ :

Theorem 4.4.2. For any graph $G, \operatorname{ch}_{\Sigma}^{v}(G) \leq \Delta(G)^{2}+1$.

Proof. Without loss of generality, assume that $G$ is connected. The proof is by induction on $|V(G)|$. The statement is true if $|V(G)| \leq 3$. Let $G$ be a graph on at least 4 vertices and let $v \in V(G)$ be any vertex. By induction, there exists a vertex weighting of $G-v$ by lists which is an additive colouring of $G-v$. Note that the 
sum which colours $v$ is now determined, and choosing $w(v)$ only changes the colour of the vertices $u \in N(v)$. Since each $u \in N(v)$ has at most $\Delta(G)$ neighbours in $G$, there are at most $\Delta(G)^{2}$ forbidden values for $w(v)$ and so an admissible choice for $w(v)$ exists.

Note that, if $G$ is chordal, then this bound can further be improved.

Theorem 4.4.3. If $G$ is a chordal graph, then $\operatorname{ch}_{\Sigma}^{v}(G) \leq \Delta(G)(\chi(G)-1)+1$.

Proof. Without loss of generality, assume that $G$ is connected. The proof is by induction on $|V(G)|$. The statement is true if $|V(G)| \leq 3$. Let $G$ be a graph on at least 4 vertices and let $v \in V(G)$ be a simplicial vertex. Note that $d_{G}(v) \leq \omega(G)-1$. Since $G$ is perfect, this implies that $d_{G}(v) \leq \chi(G)-1$. By induction, there exists a vertex weighting of $G-v$ by lists which is an additive colouring of $G-v$. The sum which colours $v$ is now determined, and choosing $w(v)$ only changes the colour of the vertices $u \in N(v)$. Since each $u \in N(v)$ has at most $\Delta(G)$ neighbours in $G$, there are at most $\Delta(G) d_{G}(v) \leq \Delta(G)(\chi(G)-1)$ forbidden values for $w(v)$ and so an admissible choice for $w(v)$ exists.

Finally, the theorem below shows that the Additive Colouring Conjecture is true for complete multipartite graphs, and in fact a stronger bound than conjectured is attainable for list-weightings.

Theorem 4.4.4. If $G$ is a complete multipartite graph $K_{t_{1}, t_{2}, \cdots, t_{k}}$ and $t=\min \left\{t_{i}: 1 \leq i \leq k\right\}$, then $\operatorname{ch}_{\Sigma}^{v}(G) \leq\left\lceil\frac{k-1}{t}+1\right\rceil$.

Proof. Let $V_{i}$ denote the colour class of size $t_{i}$. Let $\mathcal{L}$ be a list-assignment of $V(G)$ such that, for each $1 \leq i \leq k$, each $v \in V_{i}$ receives a list $L_{v}$ of size $\left|L_{v}\right|=\left\lceil\frac{k-1}{t_{i}}+1\right\rceil$; note that $\left|L_{v}\right| \leq\left\lceil\frac{k-1}{t}+1\right\rceil$ for every $v \in V(G)$. 
Let $e=v_{i} v_{j} \in E(G)$, where $v_{i} \in V_{i}, v_{j} \in V_{j}$. For a list-weighting $w$ into $\mathcal{L}$, denote $w\left(V_{i}\right)=\sum_{x \in V_{i}} w(x)$. For any weighting function $w$ from $\mathcal{L}$ and resulting vertex colouring by sums $c$,

$$
\begin{aligned}
c\left(v_{i}\right) \neq c\left(v_{j}\right) & \Longleftrightarrow \sum_{x \in N_{G}\left(v_{i}\right)} w(x) \neq \sum_{y \in N_{G}\left(v_{j}\right)} w(y) \\
& \Longleftrightarrow \sum_{x \in V(G)} w(x)-w\left(V_{i}\right) \neq \sum_{y \in V(G)} w(y)-w\left(V_{j}\right) \\
& \Longleftrightarrow w\left(V_{i}\right) \neq w\left(V_{j}\right)
\end{aligned}
$$

It suffices then to find a weighting function $w$ such that $w\left(V_{i}\right) \neq w\left(V_{j}\right)$ for each $1 \leq i, j \leq k$.

The minimum number of possible sums attainable by choosing one element from $m$ lists of size $r$ is $m r-m+1$, and hence there are at least $t_{i}\left[\left(\frac{k-1}{t_{i}}+1\right)-1\right\rceil+1 \geq k$ attainable values of $w\left(V_{i}\right)$. Let $L_{i}$ denote this list of $k$ attainable values. Let $H$ be a complete graph having vertex set $V(H)=\left\{V_{1}, \ldots V_{t}\right\}$, and assign to each $V_{i}$ the list $L_{i}$. Since $\operatorname{ch}_{\Sigma}^{v}\left(K_{k}\right)=k$ by Proposition 4.4.1, there exists an additive colouring of $H$ given by some list-weighting $w^{\prime}: V(H) \rightarrow \cup_{1 \leq i \leq k} L_{k}$. For each set $V_{i} \subset V(G)$, there exists a function $w_{i}: V_{i} \rightarrow \cup_{x \in V_{i}} L_{x}$ such that $w^{\prime}\left(V_{i}\right)=\sum_{x \in V_{i}} w_{i}(x)$. Letting $w\left(v_{i}\right)=w_{i}\left(v_{i}\right)$ if $v_{i} \in V_{i}$ thus gives $w\left(V_{i}\right)=w^{\prime}\left(V_{i}\right)$ for each $1 \leq i \leq k$, and hence the desired additive colouring of $G$.

Note that in the case of regular complete $k$-partite graphs, this implies that $\operatorname{ch}_{\Sigma}^{v}(G) \leq\left\lceil\frac{\chi(G)-1}{\alpha(G)}+1\right\rceil$. This generalizes a result of Chartrand, Okamoto, and Zhang [34], who show that $\chi_{\Sigma}^{v}(G)=\left[\frac{\chi(G)-1}{\alpha(G)}+1\right\rceil$ for such graphs only in the case when each part has size $\left(\begin{array}{c}n+l-1 \\ l-1\end{array}\right)$ for positive integers $n$ and $l \geq 2$. 


\section{Chapter 5}

\section{Edge Weightings Which Colour Vertices in Digraphs by Sums}

Edge weightings which properly vertex colour graphs by sums may be extended to the realm of digraphs by defining the colour of a vertex induced by an edge weightings to be the difference between the sum of the inbound arc weights and the sum of the

outbound arc weights. Bartnicki et al. [15] ask if their Combinatorial Nullstellensatz method, explained in Section 4.1, may be extended to digraphs and thus give an alternate proof of Theorem 2.4.1, which states that $\operatorname{ch}_{\Sigma}^{e}(D) \leq 2$ for every digraph $D$. It shown in this chapter that their method can be so extended; an earlier version of this chapter appears in a paper co-authored by myself, Khatirinejad, Naserasr, Newman, and Stevens [65].

\subsection{Main Result}

A square $n \times n$ matrix $M$ with complex entries is hermitian if $M$ is its own conjugate transpose; that is, $a_{i j}=\overline{a_{j i}}$ for all $1 \leq i, j \leq n$. If $M$ is real, then it is hermitian 
if and only if it is symmetric. A square $n \times n$ hermitian matrix $M$ with complex entries is positive semi-definite if $x^{*} M x \geq 0$ for every $x \in \mathbb{C}^{n}$, where $x^{*}$ denotes the conjugate transpose of $x$. If the entries of $M$ and $x$ are real, then this is equivalent to requiring that $x^{T} M x \geq 0$. Equivalently, $M$ is positive semi-definite if all of its eigenvalues are non-negative, or if it may be written as a product $M=A^{T} A$ for some matrix $A$.

The main tool, aside from the Combinatorial Nullstellensatz, is the following classical theorem due to Schur; see [71, Theorem 2.5, p. 26] for an English presentation and proof of this theorem:

Theorem 5.1.1 (Schur [81]). If $M$ is a positive semi-definite hermitian matrix, then $\operatorname{per}(M) \geq \operatorname{det}(M)$, with equality if and only if $M$ is diagonal or $M$ has a zero row.

Theorem 5.1.2. For any digraph $D, \operatorname{ch}_{\Sigma}^{e}(D) \leq 2$.

Proof. Let $D$ be a digraph with vertex set $V(D)$ and $\operatorname{arc}$ set $A(D)$. Let $|A(D)|=m$. Assign a variable $x_{a}$ and a list $\mathcal{L}_{a}$ of size 2 to each $a \in A(D)$. To every vertex $v \in V(D)$, associate the term

$$
P_{v}=\sum_{(u, v) \in A(D)} x_{(u, v)}-\sum_{(v, u) \in A(D)} x_{(v, u)}
$$

that is, the sum of the variables of the inbound arcs minus the sum of the variables of the outbound arcs. Finally, if $P_{D}$ is defined to be the polynomial $P_{D}=\prod_{(u, v) \in A(D)}\left(P_{u}-P_{v}\right)$, then $\operatorname{ch}_{\Sigma}^{e}(D) \leq 2$ if and only if there exists an $x_{a} \in \mathcal{L}_{a}$ for every $a \in A(D)$ such that $P_{D} \neq 0$.

Each monomial in $P_{D}$ has total degree $m$. By showing that the coefficient of 
$\prod_{a \in A(D)} x_{a}$ is nonzero, the result is obtained by applying the Combinatorial Nullstellensatz.

For every $a=(u, v) \in A(D), P_{u}-P_{v}=\sum_{a^{\prime} \in A(D)} M_{a, a^{\prime}} x_{a^{\prime}}$, where $M$ is an $m \times m$ matrix indexed by the arcs of $D$ and defined by

$$
M_{(u, v),\left(u^{\prime}, v^{\prime}\right)}= \begin{cases}2 & \text { if }(u, v)=\left(u^{\prime}, v^{\prime}\right), \\ 1 & \text { if } u=u^{\prime}, v \neq v^{\prime} \text { or } u \neq u^{\prime}, v=v^{\prime} \\ -1 & \text { if } v=u^{\prime} \text { or } u=v^{\prime}, \\ 0 & \text { otherwise. }\end{cases}
$$

The polynomial $P_{D}$ is obtained by multiplying together the entries from $M \mathbf{x}$, where $\mathbf{x}$ is the vector of variables which correspond to the arcs of $D$. In the expansion of $P_{D}$, before collecting like terms, the coefficient $c$ of any term $c \prod_{a \in A(D)} x_{a}$ is thus the product of the entries of a transversal of $M$. Hence, the coefficient of $\prod_{a \in A(D)} x_{a}$ in $P_{D}$ is $\operatorname{per}(M)$.

Note that $M=X X^{T}$, where $X$ is the $m \times n$ vertex-arc incidence matrix of $D$, that is, $X_{(u, v), w}$ equals 1 if $u=w,-1$ if $v=w$, and 0 otherwise. By Schur's theorem, since $X X^{T}$ is a positive semi-definite matrix that is neither diagonal nor has a zero row, $\operatorname{per}(M)=\operatorname{per}\left(X X^{T}\right)>\operatorname{det}\left(X X^{T}\right) \geq 0$. Hence, the coefficient of $\prod_{a \in A(D)} x_{a}$ in $P_{D}$ is nonzero, and since the highest exponent of the term $\prod_{a \in A(D)} x_{a}$ is 1 , the Combinatorial Nullstellensatz states that lists of size 2 for each variable permit a choice of values for the variables such that $P_{D} \neq 0$. Therefore, $\operatorname{ch}_{\Sigma}^{e}(D) \leq 2$.

This theorem not only establishes the best possible bound on $\operatorname{ch}_{\Sigma}^{e}(G)$, but it also yields the following interesting results which are not, at first glance, weight 
choosability problems. Recall that the weighted degree of a vertex in a digraph $D$ is $d_{D}(v)=d^{+}(v)-d^{-}(v)$.

Corollary 5.1.3. Every graph $G$ has an orientation $D$ such that the weighted degrees of $D$ give a proper vertex colouring of $G$.

Proof. Let $D^{\prime}$ be any orientation of $G$. Let $\mathcal{L}_{e}=\{-1,1\}$ for each $e \in E\left(D^{\prime}\right)$ in Theorem 5.1.2. The digraph $D$ obtained from $D^{\prime}$ by swapping the direction of those arcs with weight -1 gives the desired result.

Note that in the vertex colouring guaranteed by the above result, the parity of the colour assigned to a vertex will be the same as the parity of its degree. This implies that the number of colours used to colour a $d$-regular graph via Corollary 5.1 .3 is at most $d+1$, which is one more than the number of colours guaranteed by Brooks' Theorem in the case of non-complete graphs which are not odd cycles and precisely the chromatic number if $G=K_{n}$ or $G=C_{2 n+1}$.

Corollary 5.1.4. Every digraph $D$ has a subgraph $F$ such that the weighted degrees of $F$ give a proper vertex colouring of $D$.

Proof. Let $\mathcal{L}_{e}=\{0,1\}$ for each $e \in E(D)$ in Theorem 5.1.2. 


\section{Chapter 6}

\section{List Weightings Which Distinguish Vertices by Sequences}

\subsection{Introduction}

As a natural relaxation of properly colouring vertices by sums with edge weightings, Karoński et al. [64] and Addario-Berry et al. [1] considered properly colouring vertices by multisets, since two multisets are distinct if the sums of their elements are distinct. As a further relaxation, it makes sense to consider proper colourings by sequences, since two sequences are distinct if their underlying multisets are distinct. This chapter gives the first known results on these vertex colouring edge weightings.

We must first define how to induce a sequence of weights from an edge weighting. One natural way to do this is to define an ordering of the edge set of a graph and use it to determine the order of the elements seen by a vertex. More precisely, let $E(G)=\left\{e_{1}, e_{2}, \ldots, e_{m}\right\}$ be the edge set of a graph $G, \prec$ a total order on $E(G)$,

and let $w: E(G) \rightarrow S$ be an edge weighting of $G$. For a vertex $v \in V(G)$, let $I_{v}=\left\{i: e_{i} \ni v\right\}$. A colouring of $V(G)$ from $w$ by sequences is obtained by 
constructing a sequence for each $v \in V(G)$ by taking the multiset $\left\{\left\{w\left(e_{i}\right): i \in I_{v}\right\}\right\}$ and ordering the elements according to $w\left(e_{i}\right) \prec w\left(e_{j}\right)$ if and only if $e_{i} \prec e_{j}$.

For example, consider $C_{5}$ with vertices and edges labelled as in Figure 18. We will consider two edge orderings and attempt to properly colour the vertices by sequences for each.

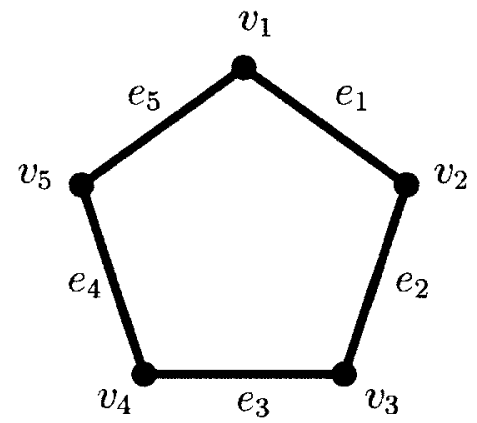

Figure 18: A labelled 5-cycle

If the edges are ordered $e_{1} \prec e_{2} \prec e_{3} \prec e_{4} \prec e_{5}$, then there is a 2-edge weighting $w: E\left(C_{5}\right) \rightarrow\{a, b\}$ which is a proper colouring by sequences, given in Table 2.

\begin{tabular}{|l|l|}
\hline Weighting & Colouring \\
\hline$w\left(e_{1}\right)=a$ & $c\left(v_{1}\right)=a a$ \\
$w\left(e_{2}\right)=b$ & $c\left(v_{2}\right)=a b$ \\
$w\left(e_{3}\right)=a$ & $c\left(v_{3}\right)=b a$ \\
$w\left(e_{4}\right)=b$ & $c\left(v_{4}\right)=a b$ \\
$w\left(e_{5}\right)=a$ & $c\left(v_{5}\right)=b a$ \\
\hline
\end{tabular}

Table 2: An edge 2-weighting that properly colours $V\left(C_{5}\right)$ by sequences

However, for the ordering $e_{1} \prec e_{3} \prec e_{5} \prec e_{2} \prec e_{4}$, the vertex colours given by a 
weighting $w$ are

$$
\begin{aligned}
& c\left(v_{1}\right)=w\left(e_{1}\right) w\left(e_{5}\right), \\
& c\left(v_{2}\right)=w\left(e_{1}\right) w\left(e_{2}\right), \\
& c\left(v_{3}\right)=w\left(e_{3}\right) w\left(e_{2}\right), \\
& c\left(v_{4}\right)=w\left(e_{3}\right) w\left(e_{4}\right), \\
& c\left(v_{5}\right)=w\left(e_{5}\right) w\left(e_{4}\right) .
\end{aligned}
$$

In order to have a proper colouring,

$$
\begin{aligned}
& w\left(e_{5}\right) \neq w\left(e_{2}\right), \\
& w\left(e_{1}\right) \neq w\left(e_{3}\right), \\
& w\left(e_{2}\right) \neq w\left(e_{4}\right), \\
& w\left(e_{3}\right) \neq w\left(e_{5}\right) .
\end{aligned}
$$

If $w$ is a weighting with only two edge weights, then inequalities (6.1.1) and (6.1.3) imply that $w\left(e_{4}\right)=w\left(e_{5}\right)$, while (6.1.2) and (6.1.4) imply that $w\left(e_{1}\right)=w\left(e_{5}\right)$. Together, this forces $c\left(v_{1}\right)=c\left(v_{5}\right)$, and hence $C_{5}$ cannot be properly vertex coloured by sequences with two edge weights for the ordering $e_{1} \prec e_{3} \prec e_{5} \prec e_{2} \prec e_{4}$.

Clearly the order of the edges plays a significant role in vertex colouring by sequences. As such, we consider the following two problems:

Problem 6.1.1. Given a graph $G$, what is the smallest value of $k$ such that there is an edge k-weighting of $G$ which is a proper colouring of $V(G)$ by sequences for some ordering of $E(G)$ ? 
Problem 6.1.2. Given a graph $G$, what is the smallest value of $k$ such that there is an edge k-weighting of $G$ which is a proper colouring of $V(G)$ by sequences for every ordering of $E(G)$ ?

These parameters will be called $\chi_{\sigma}^{e}(G)$ and $\chi_{\sigma}^{e}(G)$, respectively. For the listweighting variations, $\operatorname{ch}_{\sigma^{*}}^{e}(G)$ and $\operatorname{ch}_{\sigma}^{e}(G)$ will be used. As mentioned in the introduction, colouring by sequences is a natural relaxation of colouring by multisets (which is, in turn, a relaxation of colouring by sums). The relationship between these colouring parameters is addressed in Section 6.3.

\subsection{Colouring by sequences for some $E(G)$ ordering}

The case when one is free to choose an "optimal" ordering of the edges of a graph $G$ is the easier of the two problems to analyze. In this section, Problem 6.1.1 is solved completely for edge weightings and total weightings for graphs and multigraphs.

We begin with the simple case of cycles.

Proposition 6.2.1. If $n \geq 3, \operatorname{ch}_{\sigma^{*}}^{e}\left(C_{n}\right)=2$.

Proof. The $n=3$ case is trivial. Let $n \geq 4, V\left(C_{n}\right)=\left\{v_{1}, \ldots, v_{n}\right\}$, and $E\left(C_{n}\right)=\left\{v_{i} v_{i+1}: 1 \leq i \leq n\right\}$ with index addition taken $\bmod n$. Let $e_{i}=v_{i} v_{i+1}$. For each $i=1, \ldots, n$, let $L_{e_{i}}$ be a set of 2 elements. Choose $w\left(e_{2}\right) \in L_{e_{2}}$ and $w\left(e_{n}\right) \in L_{e_{n}}$ such that $w\left(e_{2}\right) \neq w\left(e_{n}\right)$. For each $i=3, \ldots, n-1$, let $w\left(e_{i}\right) \in L_{e_{i}} \backslash\left\{w\left(e_{i-1}\right)\right\}$ and let $w\left(e_{1}\right) \in L_{e_{1}} \backslash\left\{w\left(e_{n-1}\right)\right\}$. The resulting vertex colouring by sequences is proper.

The prefix of length $t$ of a sequence $a_{1} a_{2} \cdots a_{n}$ is the subsequence $a_{1} a_{2} \cdots a_{t}$. A vertex colouring by sequences, $c$, is prefix distinguishing if, for any $u v \in E(G)$ with 
$d(u) \geq d(v) \geq 2, c(v)$ is not the prefix of $c(u)$; in other words, if $c(v)=a_{1} a_{2} \cdots a_{k}$ and $c(u)=b_{1} b_{2} \cdots b_{l}$ for some $l \geq k$, then there exists an index $i, 1 \leq i \leq k$, such that $a_{i} \neq b_{i}$. By proving a stronger statement about prefix distinguishing colourings by sequences, one can show that $\operatorname{ch}_{\sigma^{*}}^{e}(G) \leq 2$ for every nice graph $G$. Note that we use $|S|$ to denote the length of a sequence $S$.

Proposition 6.2.2. Let $G$ be a nice graph with ordered edge set $E(G)$. If $w$ is an edge weighting which gives a prefix distinguishing colouring by sequences, then w properly colours $V(G)$ by sequences.

Proof. Let $c(v)$ be the sequence of edge weights associated with $v \in V(G)$. Consider $u v \in E(G)$. If $d(u)$ and $d(v)$ are both at least 2, then $c(u) \neq c(v)$ since $c$ is prefix distinguishing. If either $d(u)$ or $d(v)$ is 1 , say $d(u)=1$, then $d(v) \neq 1$ since $G$ is nice, and hence $c(u) \neq c(v)$ since $|c(u)|=1 \neq|c(v)|$.

Theorem 6.2.3. Let $G$ be a nice connected graph and for each $e \in E(G)$ let $L_{e}$ be a set of two real numbers. There is an ordering of $E(G)$ and values $w(e) \in L_{e}$, $e \in E(G)$, such that $w$ is a prefix distinguishing vertex colouring by sequences.

Proof. We prove the statement by induction on $|V(G)|$. The theorem is true if $|V(G)|=3$; assume $|V(G)| \geq 4$. Let $d=\delta(G)$, let $x \in V(G)$ be a vertex of minimum degree, and let $G^{\prime}=G-x$ (note that no component of $G^{\prime}$ is isomorphic to $K_{2}$ ). For various values of $d$, it will be shown that an edge weighting $w^{\prime}$ which gives a prefix distinguishing vertex colouring by sequences of $G^{\prime}$, say $c^{\prime}$, can be extended to $G$. Let $w$ and $c$ denote the extended edge weighting and vertex colouring of $G$, respectively. In each case we consider, the ordering of the edges of $E\left(G^{\prime}\right)$ which gives $c^{\prime}$ is extended by appending the edges incident to $x$ to the end of the ordering (and hence, the weights of these edges to the ends of the colours of vertices in $N_{G}(x)$ ). 
Suppose $d=1$, and let $N_{G}(x)=\{y\}$. If $d_{G^{r}}(y)=1$, let $z$ be the neighbour of $y$ in $G^{\prime}$ and choose $w(x y) \in L_{x y}$ different from the second entry in $c^{\prime}(z)$. Otherwise, $d_{G^{\prime}}(y) \geq 2$ and so, since $c^{\prime}$ is prefix distinguishing, any choice of $w(x y) \in L_{x y}$ gives a prefix distinguishing colouring of $V(G)$.

Suppose $d=2$. If $G$ is a cycle, then the result follows by Proposition 6.2.1. Assume $G$ is not a cycle. One may choose $x$ such that one of its neighbours has degree at least 3 in $G$; call this neighbour $y_{1}$. Let $y_{2}$ denote the other neighbour of $x$. There are two forbidden values of $c(x)$ given by the length 2 prefixes of $c^{\prime}\left(y_{1}\right)$ and $c^{\prime}\left(y_{2}\right)$. If $d_{G^{\prime}}\left(y_{2}\right)=1$, let $z$ denote the neighbour of $y_{2}$ in $G^{\prime}$ and choose $w\left(x y_{2}\right) \in L_{x y_{2}}$ different from the second entry in $c^{\prime}(z)$. There are then at least three possible colours for $c(x)$, and so at least one permissible choice of $w\left(x y_{1}\right) \in L_{x y_{1}}$. Suppose that $d_{G^{\prime}}\left(y_{2}\right) \geq 2$. There are at least four possible colours for $c(x)$, and so at least one permissible choice of $w\left(x y_{1}\right) \in L_{x y_{1}}$ and $w\left(x y_{2}\right) \in L_{x y_{2}}$, and hence at least two permissible choices which give the desired $c$.

Suppose $d \geq 3$, and let $N_{G}(x)=\left\{y_{1}, \ldots y_{d}\right\}$. Order $E(G)$ beginning with the edges of $E\left(G^{\prime}\right)$ as ordered by the induction hypothesis, and adding $x y_{1} \prec \ldots \prec x y_{d}$ to the end of the ordering. Since $c^{\prime}$ is prefix distinguishing in $G^{\prime}$, any choices of $w\left(x y_{i}\right) \in L_{x y_{i}}, i=1, \ldots, d$, will be prefix distinguishing in $G$ except perhaps between $x$ and some $y_{i}$. Since $\delta\left(G^{\prime}\right) \geq d-1$, the length of each sequence $c^{\prime}\left(y_{i}\right)$ is at least $d-1$. Forbid $x$ from receiving the same $(d-1)$-prefix as any of $y_{1}, y_{2}, \ldots, y_{d}$. There are $2^{d-1}$ choices for the weights of $x y_{1}, \ldots x y_{d-1}$, and hence for the prefix of length $d-1$ of $c(x)$. Since $d \geq 3$, it follows that $2^{d-1}>d$ and hence at least one $(d-1)$-prefix does not conflict with any of the $(d-1)$-prefixes of the colours assigned to $y_{1}, y_{2}, \ldots, y_{d}$. Any choice of $x y_{d} \in L_{x y_{d}}$ completes the weighting. 
Our desired result immediately follows from Proposition 6.2.2 and Theorem 6.2.3:

Corollary 6.2.4. If $G$ is a nice graph, then $\operatorname{ch}_{\sigma^{*}}^{e}(G) \leq 2$.

The following corollary is also easily deduced from Theorem 6.2.3:

Corollary 6.2.5. For any graph $G, \operatorname{ch}_{\sigma^{*}}^{t}(G) \leq 2$.

Proof. It is trivial to show that the result holds if $G$ has a connected component which is a single edge. Assume that $G$ is nice. Let $\mathcal{L}=\left\{L_{v}, L_{e}: v \in V(G), e \in E(G)\right\}$ be the set of lists assigned to the elements of $G$, and let $V(G)=\left\{v_{1}, v_{2}, \ldots, v_{n}\right\}$. Let $H$ be the graph with vertex set $V(H)=V(G) \cup\left\{u_{1}, u_{2}, \ldots, u_{n}\right\}$ and edge set $E(H)=E(G) \cup\left\{u_{1} v_{1}, u_{2} v_{2}, \ldots, u_{n} v_{n}\right\}$. Assign the list $L_{v_{i}}$ to the edge $u_{i} v_{i} \in E(H)$, and to each $e \in E(G) \cap E(H)$ assign the list $L_{e}$. By Theorem 6.2.3, there exists an ordering of $E(H)$ and a weighting function $w^{\prime}: E(H) \rightarrow \cup_{L \in \mathcal{L}} L$ such that $V(H)$ is properly coloured by sequences. Let $w$ be the weighting function on $G$ defined by $w(e)=w^{\prime}(e)$ for $e \in E(G)$ and $w\left(v_{i}\right)=w^{\prime}\left(u_{i} v_{i}\right)$ for $v_{i} \in V(G)$. By taking the ordering on $E(H)$ and replacing the edge $u_{i} v_{i}$ with the vertex $v_{i}$, one obtains an ordering on $V(G) \cup E(G)$ for which $w$ colours $V(G)$ with the same colours that $w^{\prime}$ colours $V(G) \cap V(H)$; call this colouring $c$. Since any two adjacent vertices in $G$ are adjacent in $H$, this colouring is proper.

We present an alternate proof sketch of Corollary 6.2.5. One may order $V(G) \cup E(G)$ so that the edges appear first, in an order which permits an edge 2-listweighting which gives a prefix-distinguishing vertex colouring by sequences from the given lists of potential edge weights as guaranteed by Theorem 6.2.3. By placing the vertices at the end of the ordering of $V(G)$ and choosing any vertex weights, one does not change the fact that the vertex colouring by sequences is prefix-distinguishing for 
those adjacent vertices each having degree at least 2. Furthermore, vertices of degree 1, which had sequences of length 1 as colours, are not adjacent to vertices of degree 1 and hence no colouring conflict is introduced between a degree 1 vertex and its neighbour.

Theorem 6.2.3 also easily extends to multigraphs. A multigraph is nice if it has no loopless connected component with exactly two vertices.

Theorem 6.2.6. If $M$ is a nice multigraph, then there is an ordering of $E(M)$ such that, for any assignment of lists of size 2 to the edges of $M$, there exists an edge 2-list-weighting $w$ which gives a prefix distinguishing vertex colouring by sequences.

Proof. Let $G$ be the underlying simple graph of $M$ and apply Theorem 6.2.3 to $G$; denote by $c$ the resulting colouring of $G$. The edges of $E(M) \backslash E(G)$ will be assigned to the end of the ordering of $E(G)$. For every $u v \in E(M)$ such that $d_{G}(u) \geq d_{G}(v) \geq 2$, any assignment of weights to edges in $E(M) \backslash E(G)$ preserves the prefix distinguishing vertex colouring. Consider $x \in V(G)$ with $d_{G}(x)=1$. If $d_{M}(x)=1$ as well, then any assignment of weights to the remaining edges will preserve the prefix-distinguishing vertex colouring. If $d_{M}(x) \geq 2$, then let $e \in E(M) \backslash E(G)$ be incident to $x$ and let $y$ be the other end of $e$. Choosing $w(e)$ different from the second entry in $c(y)$ preserves the prefix-distinguishing vertex colouring.

The proof of the following corollary follows exactly as the proof of Corollary 6.2.5.

Corollary 6.2.7. If $M$ is a nice multigraph, then there is an ordering of $E(M) \cup V(M)$ such that, for any assignment of lists of size 2 to the edges and vertices of $M$, there exists a total 2-list-weighting $w$ which gives a prefix distinguishing vertex colouring by sequences. 


\subsection{Colouring by sequences for any $E(G)$ ordering}

We now turn our attention to the problem of determining $\chi_{\sigma}^{e}(G), \chi_{\sigma}^{t}(G), \operatorname{ch}_{\sigma}^{e}(G)$, and $\operatorname{ch}_{\sigma}^{t}(G)$ for a graph $G$. Each bound is clearly bounded above by its multiset counterpart, e.g., $\chi_{\sigma}^{e}(G) \leq \chi_{m}^{e}(G)$. In general, it is not clear for which graphs these bounds are tight. For example, consider three small cycles $-C_{3}, C_{4}, C_{5}$.

Proposition 6.3.1. If $G=C_{3}$, then $\chi_{\sigma}^{e}(G)<\chi_{m}^{e}(G)$.

Proof. Since every vertex of $G$ has degree 2, two multiset colours of adjacent vertices are equal if and only if their sums are equal. By Proposition 3.2.9, $\chi_{\Sigma}^{e}(G)=3$ and so $\chi_{m}^{e}(G)=3$. Let $e_{1} \prec e_{2} \prec e_{3}$ be an ordering of the edges of $G$ and let $w\left(e_{1}\right)=w\left(e_{3}\right)=a$ and $w\left(e_{2}\right)=b$. The resulting sequences properly colour $V(G)$, and hence $\chi_{\sigma}^{e}(G)=2$.

Proposition 6.3.2. If $G \in\left\{C_{4}, C_{5}\right\}$, then $\chi_{\sigma}^{e}(G)=\chi_{m}^{e}(G)$.

Proof. By Proposition 3.2.9 and the discussion of the above proof, $\chi_{m}^{e}\left(C_{4}\right)=2$ and $\chi_{m}^{e}\left(C_{5}\right)=3$. Clearly $\chi_{\sigma}^{e}\left(C_{4}\right)>1$, and hence $\chi_{\sigma}^{e}\left(C_{4}\right)=2$; it was shown that $\chi_{\sigma}^{e}\left(C_{5}\right)=3$ in the introduction to the chapter.

The following proposition follows from two bounds stated in Chapter 2 $\chi_{m}^{e}(G) \leq 4$ for every nice graph (Theorem 2.2.3) and $\chi_{\Sigma}^{t}(G) \leq 3$ for every graph (Theorem 2.3.2):

Proposition 6.3.3. If a graph $G$ is nice then $\chi_{\sigma}^{e}(G) \leq 4$. For any graph $G$, $\chi_{\sigma}^{t}(G) \leq 3$

In light of the 1-2-3 and 1-2 Conjectures, the following two weaker conjectures are offered: 
Conjecture 6.3.4. If $G$ is a nice graph, then $\operatorname{ch}_{\sigma}^{e}(G) \leq 3$.

Conjecture 6.3.5. For any graph $G, \operatorname{ch}_{\sigma}^{t}(G) \leq 2$.

Conjectures 6.3.4 and 6.3.5 are verified in this section for $d$-regular graphs of sufficiently large degree and for general graphs with $\delta(G)$ sufficiently large in terms of $\Delta(G)$.

The approach in the remainder of this chapter relies on the probabilistic method. A (finite) probability space is a pair $(\Omega, \mathbf{P})$, where $\Omega$ is a (finite) set and $\mathbf{P}$ is a function $\mathbf{P}: \Omega \rightarrow[0,1]$ satisfying $\sum_{\omega \in \Omega} \mathbf{P}=1$. The probability of $\omega \in \Omega$ is $\mathbf{P}(\omega)$. An event $A$ in a probability space is a subset $A \subseteq \Omega$, and the probability of $A$ is defined to be $\mathbf{P}(A)=\sum_{\omega \in A} \mathbf{P}(\omega)$. To use the probabilistic method, one defines a probability space which contains all structures satisfying some set of conditions and if the probability of a "desirable" structure occurring is shown to be greater than zero, then that structure must exist.

For a set of events $\left\{A_{i}: i \in I\right\}$ in a probability space and a subset $K \subseteq I$, define $A_{K}:=\bigcap_{i \in K} A_{i}$ and $\bar{A}_{K}:=\bigcap_{i \in K} \bar{A}_{i}$. Let $J \subseteq I \backslash\{i\}$. The event $A_{i}$ is mutually independent of the set of events $\left\{A_{i}: i \in J\right\}$ if, for every set $J^{\prime} \subseteq J$,

$$
\mathbf{P}\left(A_{i} \cap A_{J^{\prime}}\right)=\mathbf{P}\left(A_{i}\right) \times \mathbf{P}\left(A_{J^{\prime}}\right)
$$

or, equivalently,

$$
\mathbf{P}\left(A_{i} \mid A_{J^{\prime}}\right)=\mathbf{P}\left(A_{i}\right)
$$

The main tool which will be used is the well known and powerful Lovász Local Lemma. 
Lovász Local Lemma (Erdős, Lovász [43]). Let $\left\{A_{i}: i \in I\right\}$ be events in a probability space, and for each $A_{i}$ let $J_{i} \subseteq I$ be a set of indices such that $A_{i}$ is mutually independent of $\left\{A_{j}: j \notin J_{i} \cup\{i\}\right\}$. If there exist real numbers $0<x_{i}<1$ for each $i \in I$ such that $\mathbf{P}\left(A_{i}\right)<x_{i} \prod_{j \in J_{i}}\left(1-x_{j}\right)$, then

$$
\mathbf{P}\left(\bar{A}_{I}\right) \geq \prod_{i \in I}\left(1-x_{i}\right)>0
$$

For an event $A_{i}$, the set $J_{i}$ indicated in the Lovász Local Lemma is called the dependency set of $A_{i}$. If the maximum size of a dependency set, taken over all $A_{i}$, is $D$, then setting $x_{i}=\frac{1}{D+1}$ in the Lovász Local Lemma for each $i \in I$ gives the symmetric version of the Local Lemma.

Symmetric Local Lemma (Spencer [83]). Let $\left\{A_{i}: i \in I\right\}$ be a set of events in a probability space, and for each $A_{i}$ let $J_{i} \subseteq I$ be a set of indices such that $A_{i}$ is mutually independent of $\left\{A_{j}: j \notin J_{i} \cup\{i\}\right\}$. If $\left|J_{i}\right| \leq D$ for all $i \in I$ and $\mathbf{P}\left(A_{i}\right)<\frac{1}{e(D+1)}$ for all $i \in I$, then $\mathbf{P}\left(\bar{A}_{I}\right)>0$.

Let $\left\{A_{e}: e \in E(G)\right\}$ be a set of events in a probability space which are indexed by the edge set of a graph $G$. An edge $e \in E(G)$ is covered by the event $A_{u v}$ if $e$ is adjacent to $u v$, and uncovered otherwise. Suppose that $e$ is covered by $A_{u v}$. If the event $A_{u v}$ occurs, then the weights of all edges incident to $u$ and $v$ are known, and hence one edge incident to $e$ has predetermined weight. Since the number of edges $e$ which are covered by $A_{u v}$ is bounded in terms of $\Delta(G)$, an application of the Symmetric Local Lemma gives a sufficient condition for $\operatorname{ch}_{\sigma}^{e}(G) \leq 3$.

Theorem 6.3.6. Let $G$ be a graph with minimum degree $\delta(G)=\delta$ and maximum degree $\Delta(G)=\Delta$. If $\delta>\log _{3}\left(2 \Delta^{2}-2 \Delta+1\right)+2$, then $\operatorname{ch}_{\sigma}^{e}(G) \leq 3$. 
Proof. For each $e \in E(G)$, let $L_{e}$ be a list of 3 elements associated with $e$. Fix an arbitrary ordering of $E(G)$. Choose $w(e)$ randomly from $L_{e}$ with uniform probability and let $c(u)$ denote the resulting sequence of weights of edges incident to $u \in V(G)$. For an edge $u v \in E(G)$, let $A_{u v}$ denote the event that $c(u)=c(v)$. Note that, if $d(u) \neq d(v)$, then $\mathbf{P}\left(A_{u v}\right)=0$. Suppose that $d(u)=d(v)=d$. If $u v$ occupies the same entry in the edge orderings of $u$ and $v$, then the probability of $A_{u v}$ is $\frac{1}{3^{d-1}}$. If they occupy different places, then the probability of $A_{u v}$ is $\frac{1}{3} \cdot \frac{1}{3} \cdot \frac{1}{3^{d-2}}=\frac{1}{3^{d}}$. Hence, $\mathbf{P}\left(A_{u v}\right) \leq \frac{1}{3^{\delta-1}}$.

Let $J_{u v} \subseteq E(G) \backslash\{u v\}$ be the set of edges where $j \in J_{u v}$ if and only if $A_{j}$ covers an edge incident to $u$ or $v$, and $j \neq u v$; that is, $j$ is incident to $u v$ or an edge incident to $u v$. The "bad" event $A_{u v}$ is independent of $\left\{A_{e}: e \notin J_{u v} \cup\{u v\}\right\}$, since no edge adjacent to $u v$ will have a weight detcrmincd by an event $A_{e}$ with $e \notin J_{u v}$. Hence, $D=\max \left\{\left|J_{e}\right|: e \in E(G)\right\} \leq 2(\Delta-1)+2(\Delta-1)^{2}=2 \Delta(\Delta-1)$.

The probability of a bad event $A_{u v}$ is

$$
\mathbf{P}\left(A_{u v}\right) \leq \frac{1}{3^{\delta-1}}<\frac{1}{3^{\log _{3}\left(2 \Delta^{2}-2 \Delta+1\right)+1}}<\frac{1}{e(2 \Delta(\Delta-1)+1)} \leq \frac{1}{e(D+1)} .
$$

By the Symmetric Local Lemma, $\mathbf{P}\left(\overline{A_{E(G)}}\right)>0$, and hence there exists an edge weighting such that no event $A_{u v}$ occurs; that is, an edge weighting exists which colours $V(G)$ by sequences for any ordering of $E(G)$.

If two adjacent vertices have distinct degrees, then their associated sequences will certainly differ. Hence, regular graphs are of particular interest. The following corollary is easily obtained from Theorem 6.3.6:

Corollary 6.3.7. If $G$ is a d-regular graph, $d \geq 6$, then $\operatorname{ch}_{\sigma}^{e}(G) \leq 3$. 
A similar argument gives $\operatorname{ch}_{\sigma}^{e}(G) \leq 4$ if $G$ is 5-regular, $\operatorname{ch}_{\sigma}^{e}(G) \leq 5$ if $G$ is 4-regular, and $\operatorname{ch}_{\sigma}^{e}(G) \leq 6$ if $G$ is 3-regular.

We now consider total weightings. Since the List 1-2 Conjecture implies that 2 weights should suffice for a proper colouring by sums, we consider total 2-listweightings.

Theorem 6.3.8. Let $G$ be a graph with minimum degree $\delta(G)=\delta$ and maximum degree $\Delta(G)=\Delta$. If $\delta>\log _{2}\left(e\left(2 \Delta^{2}-2 \Delta+1\right)\right)$, then $\operatorname{ch}_{\sigma}^{t}(G) \leq 2$. In particular, if $G$ is d-regular for $d \geq 9$, then $\operatorname{ch}_{\sigma}^{t}(G) \leq 2$.

Proof. The proof follows exactly as that of Theorem 6.3.6, except with $\mathbf{P}\left(A_{u v}\right) \leq \frac{1}{2^{\delta}}$. Hence,

$$
\mathbf{P}\left(A_{u v}\right) \leq \frac{1}{2^{\delta}}<\frac{1}{e(2 \Delta(\Delta-1)+1)} \leq \frac{1}{e(D+1)}
$$

and so the result follows by the Symmetric Local Lemma.

Finally, we examine $\operatorname{ch}_{\sigma}^{e}(M)$ and $\operatorname{ch}_{\sigma}^{t}(M)$ for a multigraph $M$. An application of the Local Lemma shows that as long as the maximum edge multiplicity is no more than the minimum degree less a logarithmic term in terms of maximum degree, then the bounds in Conjectures 6.3.4 and 6.3.5 can be obtained for multigraphs.

Theorem 6.3.9. Let $M$ be a loopless multigraph with maximum edge multiplicity $\mu(G)=\mu$, minimum degree $\delta(G)=\delta$, and maximum degree $\Delta(G)=\Delta$.

(1) If $\mu<\delta-1-\log _{3}\left(2 \Delta^{2}-2 \Delta+1\right)$, then $\operatorname{ch}_{\sigma}^{e}(M) \leq 3$.

(2) If $\mu<\delta-\frac{1}{2}-\log _{2}\left(2 \Delta^{2}-2 \Delta+1\right)$, then $\operatorname{ch}_{\sigma}^{t}(M) \leq 2$.

Proof. (1) Let $L_{e}$ be a list of 3 elements associated with the edge $e$. Fix an arbitrary ordering of $E(G)$. For an edge $e$, choose its weight $w(e)$ randomly from $L_{e}$ 
with uniform probability. Let $c(u)$ denote the resulting sequence of weights of edges incident to $u \in V(G)$. For an edge $e=u v$, let $A_{e}$ denote the event that $c(u)=c(v)$ If $u, v \in V(G)$ are adjacent vertices, and $l$ is the number of edges between them, then

$$
\mathbf{P}\left(A_{u v}\right) \leq 1 / 3^{\delta-l} \leq 1 / 3^{\delta-\mu}
$$

The size of the dependency set $J_{e}$ is the number of adjacent pairs of vertices from which one vertex is of distance at most one from $u$ or $v$, and hence the maximum size of a dependency set is $D \leq 2 \Delta(\Delta-1)$. The probability of a bad event is

$$
\frac{1}{3^{\delta-\mu}}<\frac{1}{3^{\log _{3}\left(2 \Delta^{2}-2 \Delta+1\right)+1}}=\frac{1}{3(2 \Delta(\Delta-1)+1)}<\frac{1}{e(D+1)}
$$

and so the result follows by the Symmetric Local Lemma.

(2) Applying the same argument to a random total list-weighting from lists of size 2 , the probability of a bad event is

$$
\frac{1}{2^{\delta-\mu+1}}<\frac{1}{2^{\log _{2}\left(2 \Delta^{2}-2 \Delta+1\right)+\frac{3}{2}}}=\frac{1}{2^{3 / 2}(2 \Delta(\Delta-1)+1)}<\frac{1}{e(D+1)}
$$

and so the result follows by the Symmetric Local Lemma.

By considering edge $k$-weightings rather than edge $k$-list-weightings one can reduce the size of a bad event's dependency set in graphs with no short cycles, and hence obtain improved bounds.

Given an ordering of the edges of a graph $G$, denote by $e_{i}^{u}$ the $i^{\text {th }}$ edge incident to $u$ with respect to the ordering of $E(G)$. A set of events $K \subseteq\left\{A_{e}: e \in E(G) \backslash\{u v\}\right\}$ leaves the edge $u v \in E(G)$ open if at least one of $\left\{e_{i}^{u}, e_{i}^{v}\right\} \backslash\{u v\}$ is left uncovered by 
$K$ for each $1 \leq i \leq \max \{\operatorname{deg}(u), \operatorname{deg}(v)\}$

Lemma 6.3.10. Let $G$ be a graph with ordered edge set $E(G)=\left\{e_{1}, \ldots, e_{m}\right\}$. Let $w: E(G) \rightarrow[k]$ be a random edge $k$-weighting where, for each $e \in E(G), w(e)$ is chosen with uniform probability from $[k]$; denote by $c(u)$ the resulting sequence of edge weights associated with $u \in V(G)$. For an edge $u v \in E(G)$, let $A_{u v}$ be the event that $c(u)=c(v)$. If there exists a set of events $K \subseteq\left\{A_{e}: e \in E(G) \backslash\{u v\}\right\}$ such that $K$ leaves uv open, then $A_{u v}$ is mutually independent of $K$.

Proof. It suffices to prove that $\mathbf{P}\left(A_{u v} \mid K\right)=\mathbf{P}\left(A_{u v}\right)$, since any proper subset of $K$ leaves more edges adjacent to $u v$ uncovered than does $K$. If $\operatorname{deg}(u) \neq \operatorname{deg}(v)$, then $\mathbf{P}\left(A_{u v}\right)=\mathbf{P}\left(A_{u v} \mid K\right)=0$.

Assume $\operatorname{deg}(u)=\operatorname{deg}(v)=d$ and suppose that, for some $i, u v=e_{i}^{u}=e_{i}^{v}$. Clearly $\mathbf{P}\left(A_{u v}\right)=\frac{1}{k^{d-1}}$. Let

$$
\begin{gathered}
U=\left\{j: e_{j}^{u} \text { is uncovered by } K\right\} \backslash\{u v\} \\
V=\left\{j: e_{j}^{v} \text { is uncovered by } K\right\} \backslash\{u v\} .
\end{gathered}
$$

Since $u v$ is left open by $K, U \cup V=[d] \backslash\{i\}$. It follows that

$$
\mathbf{P}\left(A_{u v} \mid K\right)=\left(\frac{1}{k^{|U|}}\right)\left(\frac{k^{|U \cap V|}}{k^{|V|}}\right)=\left(\frac{1}{k^{|U|}}\right)\left(\frac{1}{k^{|V \backslash U|}}\right)=\frac{1}{k^{|U \cup V|}}=\frac{1}{k^{d-1}}=\mathbf{P}\left(A_{u v}\right)
$$

If $u v=e_{r}^{u}=e_{s}^{v}$ for some $r \neq s$, then $\mathbf{P}\left(A_{u v}\right)=\frac{1}{k^{d-2}} \times \frac{k}{k^{3}}=\frac{1}{k^{d}}$. Again, let $U=\left\{j: e_{j}^{u}\right.$ is uncovered by $\left.K\right\} \backslash\{u v\}$ and $V=\left\{j: e_{j}^{v}\right.$ is uncovered by $\left.K\right\} \backslash\{u v\}$. 
Since $u v$ is left open by $A_{K}, r \in V$ and $s \in U$, and so

$$
\begin{aligned}
\mathbf{P}\left(A_{u v} \mid K\right) & =\left(\frac{1}{k^{|U \backslash\{s\}|}}\right)\left(\frac{k^{|U \cap V \backslash\{r, s\}|}}{k^{|V \backslash\{r\}|}}\right) \mathbf{P}\left(w(u v)=w\left(e_{r}^{u}\right)=w\left(e_{s}^{v}\right)\right) \\
& =\left(\frac{1}{k^{\mid U \backslash \backslash\{s\}}}\right)\left(\frac{1}{k^{|V \backslash U \backslash\{r\}|}}\right)\left(\frac{1}{k^{2}}\right) \\
& =\frac{1}{k^{|U U V \backslash\{r, s\}|}}\left(\frac{1}{k^{2}}\right) \\
& =\frac{1}{k^{d-2}} \times \frac{1}{k^{2}}=\mathbf{P}\left(A_{u v}\right),
\end{aligned}
$$

as desired.

Note the need for an edge $k$-weighting rather than an edge $k$-list-weighting in Lemma 6.3.10; it provides equality between $\mathbf{P}\left(A_{u v}\right)$ and $\frac{1}{k^{d-1}}$ when $u v=e_{i}^{u}=e_{i}^{v}$ for some index $i$, which is required to show that $\mathbf{P}\left(A_{u v} \mid K\right)=\mathbf{P}\left(\Lambda_{u v}\right)$.

Theorem 6.3.11. Let $G$ be a graph with minimum degree $\delta(G)=\delta$ and maximum degree $\Delta(G)=\Delta$ and girth at least 5. If $\delta>\log _{3}\left(\Delta^{2}-\Delta+1\right)+2$ then $\chi_{\sigma}^{e}(G) \leq 3$.

Proof. Fix an arbitrary ordering of $E(G)$ and for an edge $e$, choose its weight $w(e)$ randomly from $\{1,2,3\}$ with uniform probability. Let $c(u)$ denote the resulting sequence of weights of edges incident to $u \in V(G)$.

For an edge $u v \in E(G)$, let $A_{u v}$ denote the event that $c(u)=c(v)$. Let $J(u v)$ be the set of edges of distance at most 1 from $u$ not incident to $v$, and let $L(u v)=E(G) \backslash J(u v) \backslash\{u v\}$. Since the girth of $G$ is at least 5 , the distance from $u$ to any end of an edge is $L(u v)$ is at least 2. This implies that all edges incident to $u$ except $u v$ are left uncovered by the events $\left\{A_{l}: l \in L(u v)\right\}:=K_{u v}$, and hence $u v$ is left open by $K_{u v}$. By Lemma 6.3.10, this implies that $A_{u v}$ is mutually independent of $K_{u v}$; let $J_{u v}=J(u v)$ be the dependency set for $A_{u v}$. Since the maximum size of a 
dependency set is $D=\max \left\{\left|J_{e}\right|: e \in E(G)\right\} \leq(\Delta-1)+(\Delta-1)^{2}=\Delta(\Delta-1)$,

$$
\mathbf{P}\left(A_{u v}\right) \leq \frac{1}{3^{\delta-1}}<\frac{1}{3^{\log _{3}\left(\Delta^{2}-\Delta+1\right)+1}}<\frac{1}{e(\Delta(\Delta-1)+1)} \leq \frac{1}{e(D+1)}
$$

and so the result holds by the Symmetric Local Lemma.

A $(d, g)$-cage is a $d$-regular graph with girth $g$. Theorem 6.3 .11 implies that, for most cages, three edge weights suffice for adjacent vertices to receive distinct sequences.

Corollary 6.3.12. If $G$ is a $(d, g)$-cage with $d \neq 4$ and $g \geq 5$, then $\chi_{\sigma}^{e}(G) \leq 3$.

Proof. If $d=2$, then $G$ is a cycle of length $g$ and $\chi_{\sigma}^{e}(G) \leq \chi_{\Sigma}^{e}(G) \leq 3$ by Theorem 3.2.9. If $d=3$, then $G$ is 3-colourable (by Brook's Theorem). By Theorem 2.1.1, $\chi_{\Sigma}^{e}(G) \leq 3$ and so $\chi_{\sigma}^{e}(G) \leq 3$. If $d \geq 5$, then $d>\log _{3}\left(d^{2}-d+1\right)+2$, and the result follows from Theorem 6.3.11.

Recall that $\chi_{m}^{e}(G) \leq 3$ if $\delta(G) \geq 1000$ (Corollary 2.2.4). As such, the only graphs for which it remains to show that $\chi_{\sigma}^{e}(G) \leq 3$ holds are those with small minimum degree (at most 1000) and comparatively large maximum degree $\left(\Omega\left(3^{\delta(G) / 2}\right)\right)$

The other theorems from this section have similar relaxations.

Theorem 6.3.13. Let $G$ be a graph with minimum degree $\delta(G)=\delta$, maximum degree $\Delta(G)=\Delta$, and girth at least 5. If $\delta>\log _{2}\left(e\left(\Delta^{2}-\Delta+1\right)\right)$, then $\chi_{\sigma}^{t}(G) \leq 2$. In particular, if $G$ is d-regular for $d \geq 7$, then $\chi_{\sigma}^{t}(G) \leq 2$.

Proof. The proof follows exactly as that of Theorem 6.3 .11 , except with $\mathbf{P}\left(A_{u v}\right) \leq \frac{1}{2^{\delta}}$. Hence,

$$
\mathbf{P}\left(A_{u v}\right) \leq \frac{1}{2^{\delta}}<\frac{1}{e(\Delta(\Delta-1)+1)} \leq \frac{1}{e(D+1)}
$$


and so the result follows by the Symmetric Local Lemma.

Theorem 6.3.14. Let $M$ be a loopless multigraph with maximum edge multiplicity $\mu(G)=\mu$, minimum degree $\delta(G)=\delta$, maximum degree $\Delta(G)=\Delta$, and girth at least 5.

(1) If $\mu<\delta-1-\log _{3}\left(\Delta^{2}-\Delta+1\right)$, then $\chi_{\sigma}^{e}(M) \leq 3$.

(2) If $\mu<\delta-\frac{1}{2}-\log _{2}\left(\Delta^{2}-\Delta+1\right)$, then $\chi_{\sigma}^{t}(M) \leq 2$.

The proof is omitted; it is precisely the proof of Theorem 6.3 .9 with $D=\Delta(\Delta-1)$ instead of $D=2 \Delta(\Delta-1)$.

\subsection{Sequence irregularity strength}

Recall that the irregularity strength of a graph $G$ is the smallest integer $k$ such that $G$ has an edge $k$-weighting giving every vertex in $G$ a distinct sum of incident edge weights. The specific sequence irregularity strength of $G$, denoted $\mathrm{s}_{\sigma^{*}}^{e}(G)$, is the smallest $k$ such that there exists an ordering of $E(G)$ and an edge $k$-weighting of $G$ such every vertex receives a distinct induced sequence of incident edge weights. The general sequence irregularity strength of $G$, denoted $\mathrm{s}_{\sigma}^{e}(G)$, is the smallest $k$ such that for every ordering of $E(G)$ there exists an edge $k$-weighting of $G$ such every vertex receives a distinct induced sequence of incident edge weights.

Each "irregularity strength type" parameter has the usual natural list variant rather than each edge receiving a weight from $\{1,2, \ldots, k\}$, each is weighted from its own independently assigned list of $k$ weights. The general sequence listirregularity strength of a graph $G$ is denoted $\operatorname{ls}_{\sigma}^{e}(G)$; the other parameters are 
extended similarly. As with the 1-2-3 Conjecture variations, one could weight the vertices of $G$ as well as the edges; the corresponding parameters have " $t$ " in place of "e" in the superscript (e.g. $\operatorname{ls}_{\sigma}^{t}(G)$ for total list-weightings which distinguish vertices by sequences for any ordering of $E(G))$.

Let $n_{i}$ denote the number of vertices of degree $i$ in a graph $G$, and let $M_{G}=\max \left\{\left[n_{i}^{1 / i}\right]: 1 \leq i \leq \Delta(G)\right\}$. We make the following conjectures, which motivate the results that follow:

Conjecture 6.4.1. If $G$ is a nice graph, then $\mathrm{s}_{\sigma}^{e}(G)=M_{G}$.

Conjecture 6.4.2. If $G$ is a nice graph, then $\operatorname{ls}_{\sigma}^{e}(G)=M_{G}$.

It can be easily seen that $M_{G}$ is a lower bound on both $\mathrm{s}_{\sigma}^{e}(G)$ and $\operatorname{ls}_{\sigma}^{e}(G)$ by considering $\mathrm{s}_{\sigma^{*}}^{e}(G)$ (which bounds each parameter from below).

Proposition 6.4.3. For any nice graph $G, \mathrm{~s}_{\sigma^{*}}^{e}(G) \geq M_{G}$.

Proof. Let $w$ be an edge $k$-weighting of $G$. There are at most $k^{d}$ sequences which can be constructed from $\{1,2, \ldots, k\}$. For $w$ to distinguish vertices by sequences, we must have $k^{d} \geq n_{d}$ for each $\delta(G) \leq d \leq \Delta(G)$; the result immediately follows.

Theorem 2.6.4, due to Burris and Schelp, implies that $\mathrm{s}_{s}^{e}(G) \leq C M_{G}$ for some constant which relies only on $\Delta(G)$; together with Proposition 6.4.3, this proves that $\mathrm{s}_{\sigma}^{e}(G) \in \Theta\left(M_{G}\right)$ for graphs with bounded maximum degree.

Corollary 6.4.4. If $G$ is a nice graph, then $M_{G} \leq \mathrm{s}_{\sigma^{*}}^{e}(G) \leq \mathrm{s}_{\sigma}^{e}(G) \leq C M_{G}$ where $C$ depends only on $\Delta(G)$.

Theorem 2.6.3, due to Aigner, Triesch, and Tuza, states that $\mathrm{s}_{m}^{e}(G) \leq(5 e(d+1) ! n)^{1 / d}$, which gives an improved constant $C$ for $d$-regular 
graphs. In fact, it follows quite easily from the proof details (see [5]) that their bound holds for $\mathrm{ls}_{m}^{e}(G)$. By directly considering colouring by sequences, these bounds can be further improved.

Theorem 6.4.5. If $G$ is a nice $d$-regular graph, then $\operatorname{ls}_{\sigma}^{e}(G) \leq\left\lceil(2 e(d+1)(n-d))^{1 / d-1}\right\rceil$.

Proof. Fix an arbitrary ordering of $E(G)$. Let $L_{e}$ be a set of size $k=\left\lceil(2 e(d+1)(n-d))^{1 / d-1}\right\rceil$ associated with the edge $e$; choose its weight $w(e)$ randomly from $L_{e}$ with uniform probability. Let $c(u)$ denote the resulting sequence of weights of edges incident to $u \in V(G)$.

For an edge $e=u v$, let $A_{e}$ denote the event that $c(u)=c(v)$. By the same argument in the proof of Theorem 6.3.6, $\mathrm{P}\left(A_{e}\right) \leq 1 / k^{d-1}$. For a non-adjacent pair of vertices $p=\{u, v\}, \mathbf{P}\left(A_{p}\right) \leq 1 / k^{d}$ where $A_{p}$ is the event that $c(u)=c(v)$.

The size of a dependency set $J_{e}$ for an edge $e=u v$ is the number of edges of distance at most one from $e$ plus the number of nonadjacent pairs of vertices containing $u, v$, or a neighbour of $u$ or $v$; in other words, the total number of pairs of vertices containing at least one vertex in $N(u) \cup N(v)$. Hence,

$$
\left|J_{e}\right| \leq\left(\begin{array}{l}
n \\
2
\end{array}\right)-\left(\begin{array}{c}
n-2 d \\
2
\end{array}\right)=d(2 n-2 d-1)
$$

Similarly, the size of $J_{p}$ is

$$
\left|J_{p}\right| \leq\left(\begin{array}{l}
n \\
2
\end{array}\right)-\left(\begin{array}{c}
n-2 d-2 \\
2
\end{array}\right)=(d+1)(2 n-2 d-3) .
$$


The probability of a bad event $A \in\left\{A_{e}, A_{p}: e \in E(G), p \in(V(G) \times V(G)) \backslash E(G)\right\}$ is

$$
\mathbf{P}(A) \leq \frac{1}{k^{d-1}} \leq \frac{1}{2 e(d+1)(n-d)}<\frac{1}{e\left(\max \left\{\left|J_{e}\right|,\left|J_{p}\right|\right\}+1\right)}
$$

and so the result holds by the Symmetric Local Lemma.

A bound for total list weightings is similarly obtained:

Theorem 6.4.6. For any d-regular graph $G, d \geq 2, \operatorname{ls}_{\sigma}^{t}(G) \leq\left\lceil(2 e(d+1)(n-d))^{1 / d}\right\rceil$.

The proof is precisely the same as that of Theorem 6.4.5, with $\mathbf{P}(A) \leq \frac{1}{k^{d}}$ instead of $\mathbf{P}(A) \leq \frac{1}{k^{d-1}}$.

The theorems above are motivated by Theorem 2.6.3, which considers $d$-regular graphs. However, they easily generalize to graphs with arbitrary maximum and minimum degrees. The proofs are omitted but follow exactly as those of Theorems 6.4.5 and 6.4.6.

Theorem 6.4.7. If $G$ is a graph with minimum degree $\delta(G)=\delta$ and maximum degree $\Delta(G)=\Delta$, then

$$
\operatorname{ls}_{\sigma}^{e}(G) \leq\left\lceil(2 e(\Delta+1)(n-\Delta))^{1 / \delta-1}\right\rceil \text {, and } \operatorname{ls}_{\sigma}^{t}(G) \leq\left\lceil(2 e(\Delta+1)(n-\Delta))^{1 / \delta}\right\rceil \text {. }
$$

As a consequence, there is a constant bound on general sequence list irregularity strength for graphs with sufficiently large minimum degree.

Corollary 6.4.8. Let $n, k \in \mathbb{Z}^{+}$. If $G$ is a graph on $n$ vertices with minimum degree $\delta(G)=\delta>\log _{k}\left(\frac{e}{2}(n+1)^{2}+1\right)+1$, then $\operatorname{ls}_{\sigma}^{e}(G) \leq k$. 
Proof. Consider the function $f(\Delta)=(\Delta+1)(n-\Delta)=-\Delta^{2}+(n-1) \Delta+n$. Note that $f$ is maximized when $\Delta=\frac{1}{2}(n-1)$, and so $(\Delta+1)(n-\Delta) \leq \frac{1}{4}(n+1)^{2}$. Since $\delta(G)>\log _{k}\left(\frac{e}{2}(n+1)^{2}+1\right)+1$

$$
\begin{aligned}
& k^{\delta-1}>\left(\frac{e}{2}(n+1)^{2}+1\right) \\
\Longrightarrow & k>(2 e(\Delta+1)(n-\Delta))^{1 / \delta-1} \\
\Longrightarrow & k \geq\left\lceil(2 e(\Delta+1)(n-\Delta))^{1 / \delta-1}\right\rceil \geq \operatorname{ls}_{\sigma}^{e}(G) .
\end{aligned}
$$

Finally, we extend the results on irregularity strength to multigraphs; the proof follows similarly to that of Theorem 6.3.9.

Theorem 6.4.9. Let $M$ be a loopless multigraph with maximum edge multiplicity $\mu(G)=\mu$, minimum degree $\delta(G)=\delta$, and maximum degree $\Delta(G)=\Delta$. For any positive integer $k$,

(1) if $\mu<\delta-\log _{k}(2 e(\Delta+1)(n-\Delta))$, then $\operatorname{ls}_{\sigma}^{e}(M) \leq k$.

(2) if $\mu<\delta-\log _{k}(2 e(\Delta+1)(n-\Delta))+1$, then $\operatorname{ls}_{\sigma}^{t}(M) \leq k$.

Proof. (1) Let $L_{e}$ be a list of $k$ elements associated with the edge $e$. Fix an arbitrary ordering of $E(G)$. For an edge $e$, choose its weight $w(e)$ randomly from $L_{e}$ with uniform probability. Let $c(u)$ denote the resulting sequence of weights of edges incident to $u \in V(G)$.

For two vertices $u, v \in V(G)$, let $A_{\{u, v\}}$ denote the event that $c(u)=c(v)$. Let $e$ denote a pair of adjacent vertices and $p$ denote a pair of non-adjacent vertices. As in the proof of Theorem 6.4.5, the sizes of the dependency sets $J_{e}$ and $J_{p}$ are

$$
\left|J_{e}\right| \leq\left(\begin{array}{c}
n \\
2
\end{array}\right)-\left(\begin{array}{c}
n-2 \Delta \\
2
\end{array}\right)=\Delta(2 n-2 \Delta-1)
$$




$$
\left|J_{p}\right| \leq\left(\begin{array}{c}
n \\
2
\end{array}\right)-\left(\begin{array}{c}
n-2 \Delta-2 \\
2
\end{array}\right)=(\Delta+1)(2 n-2 \Delta-3)
$$

If $u, v \in V(G)$ are adjacent vertices, and $l$ is the number of edges between them, then $\mathbf{P}\left(A_{u v}\right) \leq 1 / k^{\delta-l} \leq 1 / k^{\delta-\mu}$. For a pair of non-adjacent vertices $p, \mathbf{P}\left(A_{u v}\right) \leq 1 / k^{\delta}$. Together, the probability of a bad event $A \in\left\{A_{e}, A_{p}: e \in E(G), p \in(V(G) \times V(G)) \backslash E(G)\right\}$ is

$$
\mathbf{P}(A) \leq \frac{1}{k^{\delta-\mu}}<\frac{1}{(2 e(\Delta+1)(n-\Delta)} \leq \frac{1}{e\left(\max \left\{\left|J_{e}\right|,\left|J_{p}\right|\right\}+1\right)}
$$

and so the result follows by the Symmetric Local Lemma.

(2) The proof follows exactly as that of (1), with the maximum probability of a bad event now $\mathbf{P}(A) \leq \mathbf{P}\left(A_{u v}\right) \leq 1 / k^{\delta-l+1} \leq 1 / k^{\delta-\mu+1}$. 


\section{Chapter 7}

\section{Minimally Intersecting Multisets and Injective Colourings}

In Chapter 6, we took the idea of edge weightings which properly colour vertices by multisets and modified the problem by considering colouring by sequences. This chapter is motivated by a different variation on multiset colourings. An edge weighting which colours vertices by multisets requires only that adjacent vertices have different multisets. We will consider, instead, a colouring that requires these multisets to be as different as possible.

\subsection{Motivation and definitions}

While the initial motivation for edge weightings which induce vertex multisets that are as different as possible between neighbours comes from the 1-2-3 Conjecture, one may also find motivation for this type of colouring from the apparently unrelated field of fractional colourings. A $t$-fold colouring of a graph $G$ is an assignment of $t$

distinct colours to each vertex so that adjacent vertices do not share a colour. The 
$t$-fold chromatic number of a graph $G$, denoted $\chi_{t}(G)$, is the smallest $n$ such that $G$ has a $t$-fold colouring from $[n]$, and the fractional chromatic number of a graph is $\chi_{f}(G)=\inf _{t} \frac{\chi_{t}(G)}{t}$.

These vertex colourings were first introduced by Stahl [84] under the name $t$-tuple vertex colourings (a misnomer, since no order is given to the colours at a vertex), who pointed out that such colourings are characterized by homomorphisms (defined in Section 7.3) into Kneser graphs, where the Kneser graph $K G_{n, t}$ has all $t$-subsets of $[n]$ as its vertices and any pair of disjoint subsets form an edge. Johnson and Holroyd [59] extended this concept to that of a $(t, \lambda)$ overlap colouring of a graph $G$, where each vertex is given $t$ colours and any two adjacent vertices share exactly $\lambda$ colours. The Johnson graph $J_{n, t}$ is the graph with all $t$-subsets of $[n]$ as its vertices and an edge joining any two subsets which share $t-1$ elements. As such, $(t, t-1)$ overlap colourings are characterized by homomorphisms into Johnson graphs.

Two natural relaxations of $t$-fold vertex colourings and overlap colourings arise. Allowing vertices to receive sets of colours of arbitrary size, one may require either of the following conditions to hold:

- adjacent vertices receive sets which intersect in no more than $t$ entries, or

- adjacent vertices receive sets which are distinct in at least $t$ entries.

The former colouring is called a $t$-intersecting set vertex colouring and the latter a $t$-non-intersecting set vertex colouring. In this terminology, a $t$-fold vertex colouring is also a 0 -intersecting set colouring and a $t$-non-intersecting set colouring. We similarly define a $t$-intersecting multiset vertex colouring and $t$-nonintersecting set vertex colouring, where vertices receive multisets of colours rather than sets. 
Let $w: E(G) \rightarrow\{1, \ldots, k\}$ be an edge $k$-weighting of a graph $G$ and let $m_{w}(v)=m(v)$ be the multiset of weights of edges incident to $v \in V(G)$. It is clear that $w$ cannot induce a 0 -intersecting multiset vertex colouring, since $w(u v) \in m(u) \cap m(v)$ for any edge $u v \in E(G)$. However, one could ask for the smallest $k$ such that $G$ has an edge $k$-weighting which is a 1 -intersecting multiset vertex colouring. The smallest such $k$ is denoted $\chi_{\cap}^{e}(G)$. All of the usual variations - total weightings, vertex weightings, list weightings - are denoted in the usual fashion.

Consider a third motivation - injective colourings of line graphs. A vertex colouring $c: V(G) \rightarrow[k]$ is said to be injective if $c(u) \neq c(v)$ whenever $N_{G}(u) \cap N_{G}(v)$ is nonempty. Note that injective vertex colourings are not necessarily proper. The smallest $k$ for which $G$ has an injective colouring is called the injective chromatic number, and is denoted $\chi_{i}(G)$. This parameter was introduced by Hahn, Kratochvíl, Šrán̆, and Sotteau [52]; much of what is known about the parameter may be found in [39]. An edge colouring of a graph $G$ is injective if the corresponding vertex colouring of $L(G)$ is injective. The smallest $k$ for which $G$ has an injective edge colouring is called the injective chromatic index, and is denoted $\chi_{i}^{\prime}(G)$; by definition, $\chi_{i}^{\prime}(G)=\chi_{i}(L(G))$. The results that follow on the injective chromatic index of a graph constitute the first known study of this parameter.

Note the similarities between injective edge colourings and 1-intersecting multiset vertex colouring from edge weightings. Each forbids two edges to receive the same colour (or weight) if they are the ends of a path of length 3 or are in a cycle of length 3. The difference between the two colourings/weightings is that injective edge colourings further forbid edges in a "claw" (that is, a subgraph isomorphic to $K_{1,3}$ ) to receive the same colour while 1 -intersecting multiset vertex colourings from edge 
weightings do not. The following two propositions summarize these observations:

Proposition 7.1.1. An edge weighting $w$ is a 1-intersecting multiset vertex colouring if and only if $w\left(e_{1}\right) \neq w\left(e_{2}\right)$ for any two edges $e_{1}, e_{2} \in E(G)$ for which there exists an edge $e \in E(G) \backslash\left\{e_{1}, e_{2}\right\}$ such that $e_{1} e e_{2}$ is a path or a cycle.

Proposition 7.1.2. An edge colouring $c$ is injective if and only if, $w\left(e_{1}\right) \neq w\left(e_{2}\right)$ for any two edges $e_{1}, e_{2} \in E(G)$ for which there exists an edge $e \in E(G) \backslash\left\{e_{1}, e_{2}\right\}$ such that (1) $e_{1} e e_{2}$ is a path, (2) $e_{1} e e_{2}$ is a cycle, or (3) $e_{1}, e_{2}$, e form a $K_{1,3}$ subgraph of G (not necessarily induced).

\subsection{Relation to other edge colourings}

We begin with a simple relation between these newly defined colouring parameters.

Proposition 7.2.1. If $G$ is a nice graph, then $\chi_{\cap}^{e}(G) \leq \chi_{i}^{\prime}(G)$.

Proof. Let $G$ be a nice graph with $\chi_{i}^{\prime}(G)=k$, and let $w: E(G) \rightarrow[k]$ be an injective edge colouring of $G$. We show that $w$ is also a 1-intersecting multiset vertex colouring of G. By Proposition 7.1.1, it suffices to show that for any 3-path or 3-cycle uvxy, we have $w(u v) \neq w(x y)$. Let $u v x y$ be a 3-path or 3-cycle. In $L(G), u v$ and $x y$ are adjacent to a common neighbour, specifically $v x$. Since $w$ is injective, $w(u v) \neq w(x y)$ as desired. Hence $w$ is an edge weighting from a set of size $k$ that is a 1-intersecting multiset vertex colouring of $G$. It follows that $\chi_{\cap}^{e}(G) \leq k=\chi_{i}^{\prime}(G)$.

We will see that $\chi_{\cap}^{e}(G)$ and $\chi_{i}^{\prime}(G)$ are equal for some graphs (Theorem 7.4.1), but may also differ arbitrarily (Theorem 7.4.7). 
The $k^{\text {th }}$ power of a graph $G$, denoted $G^{k}$, is a graph with vertex set $V(G)$ and an edge joining $u, v \in V\left(G^{k}\right)$ if there exists a $u v$-path in $G$ of length at most $k$.

Proposition 7.2.2. If $G$ is a graph and $H=L(G)^{2}$ is the square of the line graph of $G$, then $\chi_{i}^{\prime}(G) \leq \chi(H)$.

Proof. We may assume that $G$ is a connected graph. The statement is clearly true if $|V(G)| \leq 3$. Let $\chi(H)=k$ and let $w: V(H) \rightarrow[k]$ be a proper vertex colouring of $H$. Since $G$ is connected and $|V(G)| \geq 3$, there exists an edge $u v \in E(G)$ with two adjacent edges $f_{1}$ and $f_{2}$. Without loss of generality, $f_{1} \ni u$. If $f_{2} \ni u$, then $d_{L(G)}\left(f_{1}, f_{2}\right)=1$. If $f_{2} \ni v$, then $d_{L(G)}\left(f_{1}, f_{2}\right)=2$. By the choice of $w, w\left(f_{1}\right) \neq w\left(f_{2}\right)$, and so $w$ is an injective edge colouring, implying that $\chi_{i}^{\prime}(G) \leq k$.

Given a graph $G$, the maximum degree of a vertex in $L(G)^{2}$ is at most

$$
2(\Delta(G)-1)+2(\Delta(G)-1)(\Delta(G)-1)=2 \Delta(G)^{2}-2 \Delta(G),
$$

and so the following corollary is obtained:

Corollary 7.2.3. For any graph $G, \chi_{\cap}^{e}(G) \leq \chi_{i}^{\prime}(G) \leq 2 \Delta(G)^{2}-2 \Delta(G)+1$.

Proposition 7.2.4. If $G$ is a nice graph and $w: E(G) \rightarrow[k]$ is a proper edge colouring which is also an injective edge colouring, then $w$ is a proper vertex colouring of $L(G)^{2}$.

Proof. Let $e_{1}, e_{2} \in E(G)$ have distance at most 2 in $L(G)$. If $e_{1} e_{2} \in E(L(G))$, then $w\left(e_{1}\right) \neq w\left(e_{2}\right)$ since $w$ is a proper edge colouring of $G$. If $e_{1} e_{2} \notin E(L(G))$, then there exists a path of length 2 connecting $e_{1}$ and $e_{2}$ in $L(G)$. Let $f \in V(L(G))=E(G)$ be the internal vertex of this path; since $f \in N_{L(G)}\left(e_{1}\right) \cap N_{L(G)}\left(e_{2}\right)$, the injectivity of $w$ 
implies that $w\left(e_{1}\right) \neq w\left(e_{2}\right)$. Thus, $e_{1}$ and $e_{2}$ receive distinct colours from $w$ and so $w$ is a proper vertex colouring of $L(G)^{2}$.

Corollary 7.2.5. If $G$ is a graph with $\delta(G) \geq 3$, then $\chi_{i}^{\prime}(G)=\chi\left(L(G)^{2}\right)$.

Proof. We first show that $\chi_{i}^{\prime}(G) \geq \chi\left(L(G)^{2}\right)$. Let $w$ be an injective edge colouring of $G$, and consider two adjacent edges $u v, u z \in E(G)$. Since $\delta(G) \geq 3$, there exists a vertex $x$ distinct from $\{v, z\}$ such that $u x \in E(G)$. Since $u x \in N_{L(G)}(u v) \cap N_{L(G)}(u z)$, it follows that $w(u v) \neq w(u z)$ by the injectivity of $w$. Hence, $w$ is a proper edge colouring of $G$ and so, by Proposition 7.2.4, $w$ is a proper vertex colouring of $L(G)^{2}$. Hence, $\chi_{i}^{\prime}(G) \geq \chi\left(L(G)^{2}\right)$. By Proposition 7.2.2, $\chi_{i}^{\prime}(G) \leq \chi\left(L(G)^{2}\right)$, and hence $\chi_{i}^{\prime}(G)=\chi\left(L(G)^{2}\right)$.

Proposition 7.2.6. If $G$ is a nice graph, then $\chi_{\Gamma}^{e}(G) \leq\left(\begin{array}{c}\chi\left(G^{2}\right) \\ 2\end{array}\right)$.

Proof. Let $G$ be a nice graph with $\chi\left(G^{2}\right)=k$, and let $c: V\left(G^{2}\right) \rightarrow[k]$ be a proper vertex colouring of $G^{2}$. Define an edge weighting $w$ of $G$ by $w(u v)=\{c(u), c(v)\}$ for all $u v \in E(G)$. We show that $w$ is a 1-intersecting multiset vertex colouring of G. By Proposition 7.1.1, it suffices to show that for any 3-path or 3-cycle uvxy, we have $w(u v) \neq w(x y)$. Let $u v x y$ be a 3-path or 3-cycle. Since $u v x$ is a 2-path, $c(x) \notin\{c(u), c(v)\}$ and hence $w(u v)=\{c(u), c(v)\} \neq\{c(x), c(y)\}=w(x y)$. Hence $w$ is an edge weighting from a set of size $\left(\begin{array}{l}k \\ 2\end{array}\right)$ that is a 1-intersecting multiset vertex colouring of $G$. It follows that $\chi_{\cap}^{e}(G) \leq\left(\begin{array}{c}\chi\left(G_{2}^{2}\right) \\ 2\end{array}\right)$.

\subsection{A homomorphism approach}

A homomorphism $\phi$ from a graph $G$ to a graph $H$ is a function from the vertex set of $G$ to the vertex set of $H, \phi: V(G) \rightarrow V(H)$, such that if $u v \in E(G)$, then 
$\phi(u) \phi(v) \in E(H)$; one writes $\phi: G \rightarrow H$ for brevity. One may also define a homomorphism between digraphs $\phi: D \rightarrow D^{\prime}$ requiring that if $(u, v) \in A(D)$, then $(\phi(u), \phi(v)) \in A\left(D^{\prime}\right)$. Since both $t$-tuple vertex colourings and $(t, \lambda)$-overlap colourings are characterizable by homomorphisms into particular graphs, we will examine 1-intersecting multiset vertex colourings and injective edge colourings through the homomorphism lens as well.

Theorem 7.3.1. Let $G$ and $H$ be graphs. If $\phi: G \rightarrow H$ is a homomorphism and $D_{H}$ is an orientation of $H$, then

$$
\chi_{\cap}^{e}(G) \leq \sum_{a \in A\left(D_{H}\right)}\left|\phi^{-1}(t(a))\right|
$$

where $t(a)$ denotes the tail of $a$ in $D_{H}$.

Proof. Note that $G$ naturally inherits an orientation from $D_{H}$, say $D_{G}$, where $(u, v) \in A\left(D_{G}\right)$ if and only if $(\phi(u), \phi(v)) \in A\left(D_{H}\right)$.

Consider an edge $e=u v \in E(G)$ and its image $\phi(e)=\phi(u) \phi(v) \in E(H)$. If $a$ is the orientation of $\phi(e)$ in $D_{H}$, then one of $\phi(u), \phi(v)$ is $t(a)$; without loss of generality, say $t(a)=\phi(u)$, and so $u$ is the tail of $e$ 's orientation in $D_{G}$, which is denoted $t(e)$.

Define an edge weighting $w$ by $w(e)=(\phi(e), t(e))$. We show that $w$ is a 1intersecting multiset vertex colouring of $G$. By Proposition 7.1.1, it suffices to show that for any 3-path or 3-cycle $u v x y$, we have $w(u v) \neq w(x y)$. Let $u v x y$ be a 3-path or 3-cycle, and suppose that $w(u v)=w(x y)$. This implies that (1) $\phi(u x)=\phi(v y)$, and (2) $t(u x)=t(v y)$. If $t(u x)=t(v y)$, then $x=y$. This implies that $\phi(u) \phi(x)=\phi(u x)=\phi(v x)=\phi(v) \phi(x)$, and in turn this implies that $\phi(u)=\phi(v)$, a contradiction of the fact that $\phi$ is a homomorphism and that $H$ is loopless. Hence $w$ 
is an edge weighting which is a 1-intersecting multiset vertex colouring of $G$.

We now determine the number of weights given by $w$. If two edges $e_{1}, e_{2} \in E(G)$ have $\phi\left(e_{1}\right)=\phi\left(e_{2}\right)=f$, and this edge $f \in E(H)$ is oriented as $a \in A\left(D_{H}\right)$, then $w\left(e_{1}\right)$ differs from $w\left(e_{2}\right)$ only if $t\left(e_{1}\right) \neq t\left(e_{2}\right)$. Hence, for each $f \in E(H)$, the number of edge weights which have $f$ as their first coordinate is the number of distinct tails of edges which are mapped to $f$. Summing over all edges in $E(H)$, or equivalently over all arcs in $A\left(D_{H}\right)$, the bound stated in the theorem is obtained.

Corollary 7.3.2. Let $G$ be a k-colourable graph. If $c: V(G) \rightarrow\{0,1, \ldots, k-1\}$ is a proper $k$-colouring of $V(G)$ such that $C_{i}$ is the colour class containing all vertices coloured $i$ and $\left|C_{i-1}\right| \geq\left|C_{i}\right|$ for each $i=1, \ldots, k-1$, then $\chi_{\cap}^{e}(G) \leq \sum_{i=0}^{k-1} i\left|C_{i}\right|$.

Proof. Let $I I$ be the complete graph on $\{0,1, \ldots, k-1\}$, and let $\phi: G \rightarrow H$ be the homomorphism such that $\phi(v)=i$ if and only if $c(v)=i$. Let $D$ be the digraph on $\{0,1, \ldots, k-1\}$ with $(i, j) \in A(D)$ if and only if $i>j$ (so, $H$ is oriented as a transitive tournament). For an arc $(i, j) \in A(D)$,

$$
\left|\phi^{-1}(t((i, j)))\right|=\left|\phi^{-1}(i)\right|=\left|C_{i}\right|
$$

Since there are $i$ arcs in $D$ with tail $i$, the result follows by Theorem 7.3.1.

Corollary 7.3.3. If $G$ is 3-colourable, then $\chi_{n}^{e}(G) \leq|V(G)|$.

Proof. Let $c: V(G) \rightarrow[3]$ be a proper colouring of $V(G)$ with colour classes $C_{0}, C_{1}, C_{2}$, where $\left|C_{0}\right| \geq\left|C_{1}\right| \geq\left|C_{2}\right| . \quad$ By Corollary 7.3.2, $\chi_{\cap}^{e}(G) \leq 2\left|C_{2}\right|+\left|C_{1}\right| \leq\left|C_{2}\right|+\left|C_{1}\right|+\left|C_{0}\right|=|V(G)|$.

An alternating 3-walk in a digraph $D$ is defined to be a set of three arcs 
$\{(u, v),(w, v),(w, x)\} \subset A(D)$ (note that we allow $u=x$; see Figure 19).

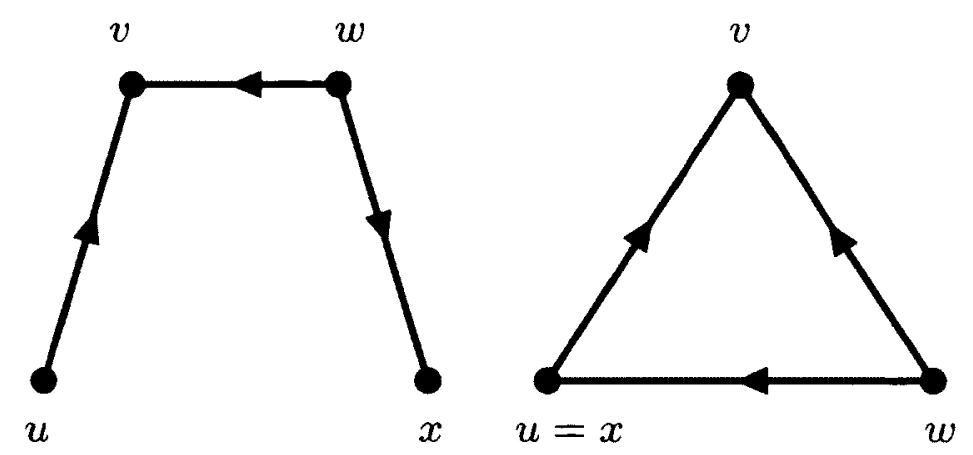

Figure 19: Two types of alternating 3-walks

Theorem 7.3.4. Let $G$ be a graph and let $D_{G}$ be an orientation of $G$ which does not contain an alternating 3-walk. If $D_{H}$ is a loopless digraph with underlying graph $H$ and there exists a homomorphism $\phi: D_{G} \rightarrow D_{H}$, then $\chi_{n}^{e}(G) \leq \chi\left(L(H)^{2}\right)$.

Proof. Let $c: E(H) \rightarrow[k]$ be an edge colouring which properly colours the vertices of $L(H)^{2}$. Noting that $\phi$ is also a homomorphism between $G$ and $H$, define $w(e)=c(\phi(e))$ for each $e \in E(G)$. Let $u v, x y \in E(G)$ be distinct edges, joined by the edge $v x \in E(G)$ (note that $u$ and $y$ need not be distinct).

Suppose that $w(u v)=w(x y)$. This implies $c(\phi(u v))=c(\phi(x y))$. If $\phi(u v)=\phi(x y)$, then $\phi(u) \phi(v)=\phi(x) \phi(y)$, and so $\phi(u)=\phi(x)$ and $\phi(v)=\phi(y)$. Without loss of generality, say that $(\phi(x), \phi(v)) \in A\left(D_{H}\right)$. This implies that $(\phi(u), \phi(v)),(\phi(x), \phi(y)) \in A\left(D_{H}\right)$, giving an alternating 3 walk in $D_{G}$ with arcs $(u, v),(x, v),(x, y)$, a contradiction. Hence $\phi(u v) \neq \phi(x y)$. However, this implies that $\phi(u v)$ and $\phi(x y)$ are two distinct edges in $H$, joined by an edge $\phi(v x) \in E(H)$, which 
receive the same colour, contradicting the choice of $c$. This gives that $w(u v) \neq w(x y)$, and so by Proposition 7.1.1, $w$ gives a 1-intersecting multiset vertex colouring.

A vertex colouring $c: V(G) \rightarrow S$ is $P_{3}$-nonrepetitive if, for any 3-path $u v w x$, either $c(u) \neq c(w)$ or $c(v) \neq c(x)$.

Corollary 7.3.5. If $G$ has a proper vertex 3 -colouring which is $P_{3}$-nonrepetitive, then $\chi_{\cap}^{e}(G) \leq 3$

Proof. Let $c: V(G) \rightarrow[3]$ be a proper vertex colouring of $G$ which is $P_{3}$-nonrepetitive. Let $D_{G}$ be the orientation of $G$ obtained by orienting $u v \in E(G)$ as $(u, v) \in A\left(D_{G}\right)$ if and only if

- $c(u)=1$ and $c(v)=2$,

- $c(u)=2$ and $c(v)=3$, or

- $c(u)=3$ and $c(v)=1$.

If $D_{G}$ has an alternating 3-walk $W=\{(u, v),(w, v),(w, x)\}$, then $c(u)=c(w)$ and $c(v)=c(x)$ by choice of $D_{G}$. This contradicts the supposition that $c$ is $P_{3^{-}}$ nonrepetitive, and hence $G$ contains no alternating 3 -walks.

Let $H$ be a complete graph on $V(H)=\{1,2,3\}$ and $D_{H}$ be a cyclic orientation on $E(H)$, with $\left.A\left(D_{H}\right)=(1,2),(2,3),(3,1)\right\}$. Clearly $L(H)$ is also isomorphic to $K_{3}$, as is its square. The mapping $\phi: D_{G} \rightarrow D_{H}$ given by $\phi(u)=c(u)$ is a homomorphism, and so by Theorem 7.3.4, $\chi_{\cap}^{e}(G) \leq \chi\left(L(H)^{2}\right)=3$.

A homomorphism $\phi: G \rightarrow H$ is locally injective if, for every $x \in V(G)$ and every distinct $u, v \in N(x), \phi(u) \neq \phi(v)$. 
Theorem 7.3.6. Let $G$ and $H$ be simple graphs. If there exists a locally injective homomorphism $\phi: G \rightarrow H$, then $\chi_{\cap}^{e}(G) \leq \chi_{\cap}^{e}(H)$.

Proof. Let $w^{\prime}: E(H) \rightarrow S$ be an edge weighting which gives a 1-intersecting multiset vertex colouring of $H$, and define $w(u v)=w^{\prime}(\phi(u v))$ for an edge $u v \in E(G)$. Consider $u v, v x, x y \in E(G)$. Since $\phi$ is locally injective, $\phi(u) \neq \phi(x)$ and $\phi(v) \neq \phi(y)$. Hence, $\phi(u v), \phi(v x), \phi(x y) \in E(H)$ are distinct edges. This gives that $w^{\prime}(\phi(u v)) \neq w^{\prime}(\phi(x y))$, and so $w(u v) \neq w(x y)$. The result follows from Proposition 7.1.1.

Theorem 7.3.7. Let $G$ and $H$ be simple graphs. If there exists a locally injective homomorphism $\phi: G \rightarrow H$, then $\chi_{i}^{\prime}(G) \leq \chi_{i}^{\prime}(H)$.

Proof. Let $w^{\prime}: E(H) \rightarrow S$ be an injective edge colouring of $H$, and define $w(u v)=w^{\prime}(\phi(u v))$ for an edge $u v \in E(G)$. Since $w^{\prime}$ is an injective edge colouring, it is also a 1-intersecting multiset vertex colouring by Theorem 7.2.1. By the proof of Theorem 7.3.6, for edges $u v, v x, x y \in E(G), w(u v) \neq w(x y)$.

Now, consider edges $u x, u y, u z \in E(G)$. Since $\phi$ is locally injective, $\phi(x) \neq \phi(y) \neq \phi(z)$, and so $\phi(u x), \phi(u y), \phi(u z) \in E(H)$ are distinct edges. This gives that $w^{\prime}(\phi(u x)), w^{\prime}(\phi(u y)), w^{\prime}(\phi(u z))$ are distinct, and so $w(u x), w(u y), w(u z)$ are distinct. By Proposition 7.1.2, $w$ is an injective edge colouring of $G$.

If $G^{\prime}$ is a subgraph of a graph $G$, then the homomorphism $\phi: G^{\prime} \rightarrow G$ defined by $\phi(v)=v$ is locally injective. This observation, together with Theorems 7.3 .6 and 7.3.7, implies that $\chi_{n}^{e}(G)$ and $\chi_{i}^{\prime}(G)$ are monotonic parameters with respect to subgraphs.

Corollary 7.3.8. If $G^{\prime}$ is a subgraph of $G$, then $\chi_{\cap}^{e}\left(G^{\prime}\right) \leq \chi_{\cap}^{e}(G)$ and $\chi_{i}^{\prime}\left(G^{\prime}\right) \leq \chi_{i}^{\prime}(G)$. 
Finally, one can prove that there is a homomorphism characterization of graphs for which $\chi_{\cap}^{e}(G)=k$ if one moves from graphs to relational systems, a concept from universal algebra. A relational system is a pair $(A, R)$ where $A$ is a set and $R$ is a $k$-ary relation on $A$ (that is, a set of $k$-tuples from $A$ ). For example, a digraph is a relational system where $R$ is a binary relation, and a graph may be thought of as a relational system where $R$ is a binary reflexive relation. As with graphs, one can consider homomorphisms between relational systems. Let $\mathcal{A}=(A, R)$ and $\mathcal{B}=(B, S)$ be relational systems with $k$-ary relations. A homomorphism between $\mathcal{A}$ and $\mathcal{B}$ is a map $\phi: A \rightarrow B$ such that $\left(\phi\left(a_{1}\right), \phi\left(a_{2}\right), \ldots, \phi\left(a_{k}\right)\right) \in S$ for all $\left(a_{1}, a_{2}, \ldots, a_{k}\right) \in R$ (see [27] for further details). Such a homomorphism is often written $\phi: \mathcal{A} \rightarrow \mathcal{B}$.

Theorem 7.3.9. Let $G$ be a graph, and define $R_{G}$ to be a 4-ary relation on $V(G)$ where $(a, b, c, d) \in R_{G}$ if and only if abcd is a 3-path in $G$ or abcd is a 3-cycle in $G$ (and so $a=d$ ). Let $S(k)$ be the set of all multisets from $[k]$ of size at most $\Delta(G)$, and define $R_{S(k)}$ to be the 4-ary relation on $S(k)$ where $(w, x, y, z) \in R_{S(k)}$ if and only if $|w \cap x|=|x \cap y|=|y \cap z|=1$ and $w \cap x \neq y \cap z$. If $\mathcal{G}=\left(V(G), R_{G}\right)$ and $\mathcal{S}_{k}=\left(S(k), R_{S(k)}\right)$, then $\chi_{n}^{e}(G)=k$ if and only if $k$ is the smallest integer such that there exists a homomorphism $\phi: \mathcal{G} \rightarrow \mathcal{S}_{k}$.

Proof. Suppose $\chi_{n}^{e}(G)=k$, and let $w$ be a $k$-edge weighting which colours $V(G)$ by 1intersecting multisets. Denote by $m(u)$ the multiset of weights from the edges incident to $u \in V(G)$, and let $\phi: \mathcal{G} \rightarrow \mathcal{S}_{k}$ be defined by $\phi(u)=m(u)$. If $a b c d$ is a 3-path in $G$, then $|m(a) \cap m(b)|=|m(b) \cap m(c)|=|m(c) \cap m(d)|=1$ and $m(a) \cap m(b) \neq m(c) \cap m(d)$ (otherwise the common element occurs in both $m(b)$ and $m(c)$ and is not $w(b c)$ ). If $a b c$ is a 3-cycle in $G$, then $|m(a) \cap m(b)|=|m(b) \cap m(c)|=|m(c) \cap m(a)|=1$ and $m(a) \cap m(b), m(a) \cap m(c)$, and $m(b) \cap m(c)$ are all distinct. Hence $\phi$ is the desired 
homomorphism.

Suppose that $\phi: \mathcal{G} \rightarrow \mathcal{S}_{k}$ is the desired homomorphism. For each $e=u v \in E(G)$, let $w(e)=\phi(u) \cap \phi(v)$. Let $e_{1}=u_{1} v_{1}$ and $e_{2}=u_{2} v_{2}$ be two edges with $u_{1}$ adjacent to $u_{2}$. Clearly $v_{1} u_{1} u_{2} v_{2}$ is either a 3 -path or 3 -cycle in $G$. If $w\left(e_{1}\right)=w\left(e_{2}\right)$, then $\phi\left(u_{1}\right) \cap \phi\left(v_{1}\right)=\phi\left(u_{2}\right) \cap \phi\left(v_{2}\right)$, contradicting the fact that $\left(\phi\left(v_{1}\right), \phi\left(u_{1}\right), \phi\left(u_{2}\right), \phi\left(v_{2}\right)\right) \in R_{S}$. Hence $w\left(e_{1}\right) \neq w\left(e_{2}\right)$ for all such pairs of edges, and so $w$ gives a 1-intersecting multiset vertex colouring by Proposition 7.1.1.

Understanding when such homomorphisms exist is likely no small task, but the extension of this weighting problem into the realm of universal algebra may hopefully open up new avenues of exploration.

\subsection{Parameter values for classes of graphs}

We first consider the simple case of complete graphs, where we determine that the bound given by Theorem 7.3.1 is in fact the exact value of $\chi_{n}^{e}(G)$. Combined with previous results, we obtain a lower bound on $\chi_{\cap}^{e}(G)$ and $\chi_{i}^{\prime}(G)$ in terms of the clique number of $G$.

Theorem 7.4.1. If $G=K_{n}, n \geq 2$, then $\chi_{\cap}^{e}(G)=\chi_{i}^{\prime}(G)=\left(\begin{array}{l}n \\ 2\end{array}\right)$.

Proof. Assigning each edge of $G$ a unique colour clearly gives an injective edge colouring, and so $\chi_{i}^{\prime}(G) \leq\left(\begin{array}{c}n \\ 2\end{array}\right)$. For any two distinct edges $e_{1}, e_{2}$, one end of $e_{1}$ must be adjacent to an end of $e_{2}$, and hence $e_{1}$ and $e_{2}$ must receive distinct weights to achieve a 1-intersecting multiset vertex colouring. Thus $\chi_{\cap}^{e}(G) \geq\left(\begin{array}{l}n \\ 2\end{array}\right)$, and by Proposition $7.2 .1,\left(\begin{array}{l}n \\ 2\end{array}\right) \leq \chi_{\cap}^{e}(G) \leq \chi_{i}^{\prime}(G) \leq\left(\begin{array}{c}n \\ 2\end{array}\right)$. 
A lower bound on $\chi_{\Gamma}^{e}(G)$ can now by obtained by applying Corollary 7.3 .8 to a maximal complete subgraph of a graph $G$.

Corollary 7.4.2. For any nice graph $G,\left(\begin{array}{c}\omega(G) \\ 2\end{array}\right) \leq \chi_{\cap}^{e}(G) \leq \chi_{i}^{\prime}(G)$.

We now turn our attention to complete multipartite graphs where, unlike with complete graphs, the values of $\chi_{n}^{e}(G)$ and $\chi_{i}^{\prime}(G)$ may vastly differ. First, consider the case of edge weightings which are 1-intersecting multiset vertex colourings. As with complete graphs, we find that $\chi_{\cap}^{e}\left(K_{m, n}\right)$ meets the bound given in Theorem 7.3.1.

Theorem 7.4.3. If $G=K_{m, n}, m, n \in \mathbb{Z}^{+}$, then $\chi_{n}^{e}(G)=\min \{m, n\}$.

Proof. Let $C_{0} \cup C_{1}$ be the bipartition of $V(G)$ into stable sets of size $m$ and $n$, respectively; without loss of generality, assume $m \geq n$. By Corollary 7.3.2, $\chi_{\cap}^{e}(G) \leq 0 \times\left|C_{0}\right|+1 \times\left|C_{1}\right|=n$. Now, suppose that $\chi_{n}^{e}(G)<n$. Since each vertex in $C_{0}$ has $n$ neighbours, at least two edges incident to each vertex in $C_{0}$ must receive the same weight; for each $v_{i} \in C_{0}, 1 \leq i \leq m$, let $w_{i}$ be such a weight. Since $m \geq n$, there must exist two vertices $v_{r}, v_{s}$ such that $w_{r}=w_{s}$. Denote by $v_{r} x_{r}, v_{r} y_{r}, v_{s} x_{s}, v_{s} y_{s}$ four edges which have the same weight $w_{r}=w_{s}=w$. Clearly $x_{r}$ must be distinct from either $x_{s}$ or $y_{s}$; without loss of generality, say that $x_{r} \neq x_{s}$. It follows that $v_{r} x_{s} \in E(G)$ and each of $v_{r}$ and $x_{s}$ are incident to an edge weighted $w$ which is not $v_{r} x_{s}$, a contradiction of Proposition 7.1.1. Thus $\chi_{n}^{e}(G)=n=\min \{m, n\}$.

Theorem 7.4.4. Let $k \geq 3$ be an integer, $n_{0}, n_{1}, \ldots, n_{k-1} \in \mathbb{Z}^{+}$. If $G=K_{n_{0}, n_{1}, \cdots, n_{k-1}}$ and $n_{0} \geq n_{1} \geq \cdots \geq n_{k-1}$, then $\chi_{\cap}^{e}(G)=\sum_{i=0}^{k-1} i n_{i}$.

Proof. Let $\left\{C_{0}, C_{1}, \ldots, C_{k-1}\right\}$ be a $k$-partition of $V(G)$ into stable sets with $\left|C_{i}\right|=n_{i}$, $0 \leq i \leq k-1$, and let $G_{i j}=G\left[C_{i} \cup C_{j}\right]$. Note that the set $\left\{G_{i j}: 0 \leq i, j \leq k-1\right\}$ 
induces an edge partition of $G$. Let $w$ be a 1-intersecting multiset vertex colouring of $G$. The sets $\left\{w(e): e \in G_{i j}\right\}$ and $\left\{w(e): e \in G_{k l}\right\}$ are disjoint for any distinct $G_{i j}$ and $G_{k l}$, since any edges $e \in E\left(G_{i j}\right)$ and $f \in E\left(G_{k l}\right)$ are connected by a distinct edge in $G$. This implies that $\chi_{n}^{e}(G) \geq \sum_{i=0}^{k-1} \sum_{j=0}^{i-1} \chi_{\cap}^{e}\left(G_{i j}\right)$, and so, by Theorem 7.4.3, $\chi_{\cap}^{e}(G) \geq \sum_{i=0}^{k-1} \sum_{j=0}^{i-1} n_{i}=\sum_{i=0}^{k-1} i n_{i}$. By Theorem 7.3.2, $\chi_{\cap}^{e}(G) \leq \sum_{i=0}^{k-1} i n_{i}$, and so $\chi_{n}^{e}(G)=\sum_{i=0}^{k-1} i n_{i}$.

Now, we consider the case of injective edge colourings.

Theorem 7.4.5. If $G=K_{2, n}$, then $\chi_{i}^{\prime}(G)=n$.

Proof. Let $u, v \in V(G)$ be the two vertices of degree $n$. If $n=2$, then $G$ is a 4-cycle and $\chi_{i}^{\prime}(G)=2$ is obtained by assigning $w(e)=a$ to the edges incident to $u$ and $w(e)=b$ to the edges incident to $v$. Suppose $n \geq 3$. Any pair of edges incident to $u$ have a common adjacent edge, and hence every edge incident to $u$ must receive a distinct colour. This implies that $\chi_{i}^{\prime}(G) \geq n$.

Let $x_{1}, x_{2}, \ldots, x_{n}$ be the vertices of degree 2 , and assign the weights $w(e)=i$ if $e$ is incident to $x_{i}$. Take any two edges, say $e_{1}$ and $e_{2}$, adjacent to a common edge, say $e_{3}$. First suppose that all three edges share an end. They must all be incident to either $u$ or $v\left(\right.$ say $u$ ). Hence $e_{1}=u x_{i}, e_{2}=u x_{j}$, and $e_{3}=u x_{k}$ for some distinct $i, j, k \in[n]$, and so $w\left(e_{1}\right)=i, w\left(e_{2}\right)=j$ and $w\left(e_{3}\right)=k$ are all distinct. Now, suppose that $e_{1} e_{3} e_{2}$ is a path, $P$. Without loss of generality, assume $e_{3}=u x_{i}$ for some $i \in[n]$. It follows that $e_{1}=u x_{j}$ for some $j \in[n] \backslash\{i\}$ and $e_{2}=v x_{i}$, and hence $w\left(e_{1}\right)=j \neq i=w\left(e_{2}\right)$. Thus $w$ is an injective edge colouring of $G$, and $\chi_{i}^{\prime}(G)=n$.

Theorem 7.4.6. Let $k \geq 2$ be an integer, $n_{1}, n_{2}, \ldots, n_{k} \in \mathbb{Z}^{+}$, and let $G=K_{n_{1}, \cdots, n_{k}}$. If $n_{i} \geq 3$ for each $1 \leq i \leq k$, then $\chi_{i}^{\prime}(G)=\sum_{1 \leq i<j \leq k} n_{i} n_{j}$. 
Proof. Let $\left\{C_{1}, C_{2}, \ldots, C_{k}\right\}$ be a $k$-partition of $V(G)$ into stable sets with $\left|C_{i}\right|=n_{i}$, $1 \leq i \leq k$. Consider any two distinct edges of $G, e_{1}, e_{2} \in E(G)$. If $e_{1}=u v$ and $e_{2}=x y$ have ends lying in different parts of $G$, say $v \in C_{i}$ and $y \in C_{j}$, then the edge $v y$ connects $e_{1}$ and $e_{2}$; hence $e_{1}$ and $e_{2}$ must receive distinct colours. Now suppose that $e_{1}$ and $e_{2}$ connect vertices in the same parts, say $u, x \in C_{i}$ and $v, y \in C_{j}(i \neq j)$. If $e_{1}$ and $e_{2}$ are adjacent, say $u=x$, then there exists a third edge $e_{3}$ incident to $u$ and hence adjacent to $e_{1}$ and $e_{2}$. This implies that $e_{1}$ and $e_{2}$ must receive distinct colours in an injective edge colouring. If $e_{1}$ and $e_{2}$ are not adjacent, then the edge $u y$ connects $e_{1}$ and $e_{2}$ (as does $v x$ ), and so $e_{1}$ and $e_{2}$ must receive distinct colours. By exhaustion, this gives that every edge of $G$ must receive a distinct colour in an injective edge colouring of $G$, and hence $\chi_{i}^{\prime}(G)=|E(G)|=\sum_{1 \leq i<j \leq k} n_{i} n_{j}$.

Note that, if $G$ is the complete $k$-partite graph $K_{n, n, \cdots, n}(k \geq 2)$, then $\chi_{\cap}^{e}(G)=n\left(\begin{array}{c}k \\ 2\end{array}\right)$ and $\chi_{i}^{\prime}(G)=n^{2}\left(\begin{array}{c}k \\ 2\end{array}\right)$. Even though $\chi_{i}^{\prime}(G)$ and $\chi_{n}^{e}(G)$ may appear to be related at first glance, we can now see that they may differ arbitrarily even for graphs with fixed chromatic number.

Corollary 7.4.7. If $k \geq 2$ is an integer and $N$ is any positive number, then there exists a graph $G$ with $\chi(G)=k$ and $\chi_{i}^{\prime}(G)-\chi_{\cap}^{e}(G)>N$.

Proof. Let $G=K_{n, n, \cdots, n}$ be a complete $k$-partite graph with $n>\sqrt{\frac{2 N}{k^{2}-k}}+1$. Clearly $\chi(G)=k$ and, by Theorems 7.4.6 and 7.4.4,

$$
\chi_{i}^{\prime}(G)-\chi_{\cap}^{e}(G)=n^{2}\left(\begin{array}{l}
k \\
2
\end{array}\right)-n\left(\begin{array}{l}
k \\
2
\end{array}\right)>(n-1)^{2}\left(\begin{array}{l}
k \\
2
\end{array}\right)>\frac{2 N}{k^{2}-k}\left(\begin{array}{l}
k \\
2
\end{array}\right)=N .
$$

Finally, we consider $\chi_{\cap}^{e}(G)$ for a collection of other interesting classes of graphs. If $G$ is a graph with $\Delta(G) \leq 2$, then it is easy to see that $\chi_{n}^{e}(G)=\chi_{\Sigma}^{e}(G)$, and hence 
$\chi_{n}^{e}(P)=2$ for any path $P, \chi_{n}^{e}\left(C_{4 k}\right)=2$ for any $k \in \mathbb{Z}^{+}$, and $\chi_{n}^{e}\left(C_{n}\right)=3$ if $n$ is not a multiple of 4 . If $G$ is a star (that is, isomorphic to $K_{1, n}$ for some positive integer $n$ ), then it is easy to see that $\chi_{\cap}^{e}(G)=1$. Note that stars are the only graphs for which $\chi_{\cap}^{e}(G)=1$. This follows from the fact that $\chi_{\cap}^{e}(G)=1$ implies that any two edges of $G$ are adjacent, and the only such graphs are $K_{3}$ (which is shown to have $\chi_{\cap}^{e}\left(K_{3}\right)=3$ in Theorem 7.4.1) and the set of stars.

Stars and paths show that there exist trees with $\chi_{\cap}^{e}(G)=1$ and $\chi_{\cap}^{e}(G)=2$. The following result, which makes use of orientations containing no alternating 3-walk, shows that $\chi_{\cap}^{e}(G)$ is bounded by a constant for any tree:

Theorem 7.4.8. If $T$ is a tree, then $\chi_{\cap}^{e}(T) \leq 3$.

Proof. Consider $T$ as a rooted tree with root $x \in V(T)$. Let $c: V(T) \rightarrow\{0,1,2\}$ be defined by $c(v)=d(v, x)(\bmod 3)$. Consider a 3 -path $P=q r s t$. Without loss of generality, either $q$ or $r$ may be assumed to be the closest vertex of $P$ to $x$. If $q$ is the closest and $c(q)=a$, then the colours of the vertices of $P$ are, in order, $a, a+1, a+2, a$ $(\bmod 3)$. If $r$ is the closest and $c(r)=a$, then the colours of the vertices of $P$ are, in order, $a+1, a, a+1, a+2(\bmod 3)$. In either case, $c$ is $P_{3}$-nonrepetitive, and the result follows from Corollary 7.3.5.

Note that $\chi_{n}^{e}\left(K_{m, n}\right)=\min \{m, n\}$ implies that $\chi_{\cap}^{e}(G)$ can not be bounded by a constant for bipartite graphs in general. However, there are other infinite classes of bipartite graphs which do have bounded values of $\chi_{n}^{e}(G)$.

Theorem 7.4.9. If $G=C_{4 n} \square C_{4 m}, n, m \geq 1$, then $\chi_{\cap}^{e}(G)=4$.

Proof. Define $V(G)=\{(i, j): 0 \leq i \leq 4 n-1,0 \leq j \leq 4 m-1\}$ and say that two vertices are adjacent only if they differ by $1(\bmod 4)$ in precisely one component and 
are equal in the other component.

Suppose that $\chi_{\cap}^{e}(G) \leq 3$. Let $w: E(G) \rightarrow\{a, b, c\}$ be an edge 3-weighting which colours $V(G)$ by 1-intersecting multisets. Without loss of generality, assume that $w((1,0),(1,1))=a$ and $w((1,2)(1,3))=b$; see Figure 20. This implies that $w((2,1)(2,2))=c$, and in turn that $w((1,1)(1,2))=a$ or $b$; again, without loss of generality, say $w((1,1)(1,2))=a$. It follows that $w((2,2)(2,3))=c$, which implies that $w((1,3)(1,4))=b$. This implies that $w((1,2),(2,2)=c$. Consider the edge

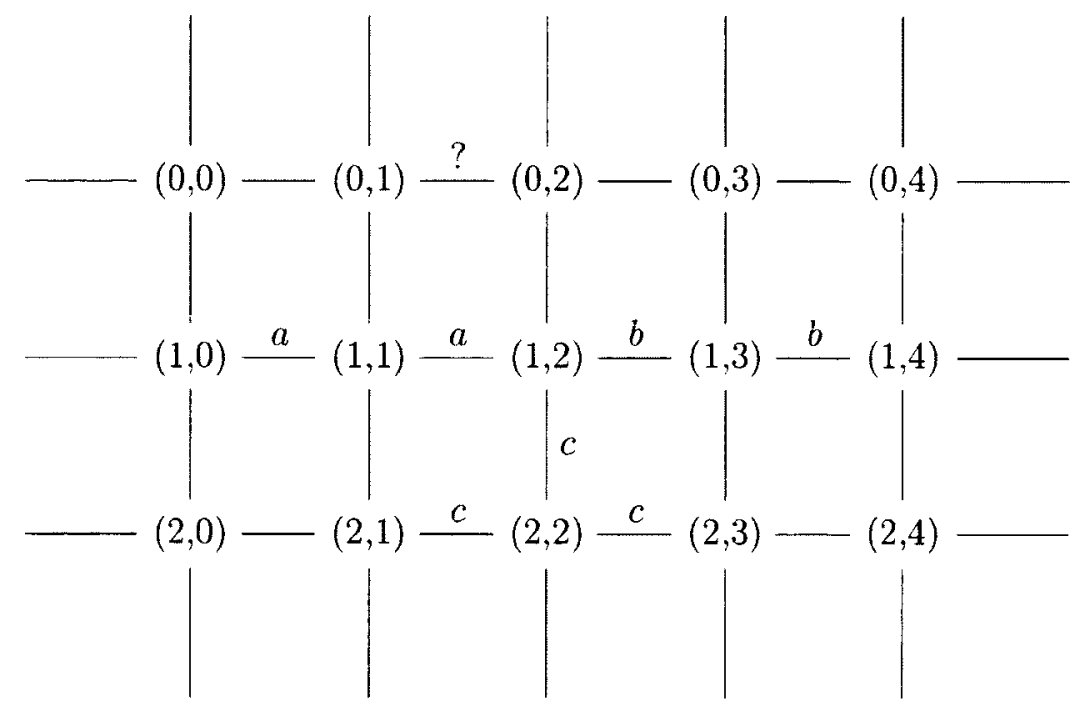

Figure 20: An edge weighting showing that $\chi_{\cap}^{e}\left(C_{4 n} \square C_{4 m}\right)>3$

$e=(0,1)(0,2)$. Since $w(e)$ must differ from the weight of any edge incident to $(1,2)$, $w(e) \neq a, b, c$, a contradiction. Hence, $\chi_{\cap}^{e}(G) \geq 4$.

To show $\chi_{\cap}^{e}(G) \leq 4$, we construct a valid edge weighting $w: E(G) \rightarrow\{a, b, c, d\}$. Every edge is incident to precisely one vertex $(i, j)$ such that $i+j$ is even. Let $e$ be 
an edge, $(i, j)$ its end with $i+j$ even, and assign $w(e)$ as follows:

$$
w(e)=\left\{\begin{array}{lll}
a & \text { if } i \text { is even and } j \equiv i & (\bmod 4) \\
b & \text { if } i \text { is even and } j \not \equiv i & (\bmod 4) \\
c & \text { if } i \text { is odd and } j \equiv i & (\bmod 4) \\
d & \text { if } i \text { is odd and } j \neq i \quad & (\bmod 4)
\end{array}\right.
$$

Let $c(v)$ denote the multiset of weights from edges incident to $v \in V(G)$. If $v=(i, j)$ with $i+j$ even, then $c(v)$ consists of 4 equal weights. If $v=(i, j)$ with $i+j$ odd, then $c(v)=\{a, b, c, d\}$. Since every edge has the form $u v=(i, j)(i, j+1)$ or $u v=(i, j)(i+1, j), c(u) \cap c(v)$ has precisely one element as desired.

The final graphs to be considered in this chapter are wheels.

Theorem 7.4.10. For any $k \in \mathbb{Z}^{+}$,

1. $\chi_{\cap}^{e}\left(W_{4 k}\right)=4$;

2. $\chi_{\cap}^{e}\left(W_{4 k+2}\right)=5$;

3. $\chi_{\cap}^{e}\left(W_{2 k+1}\right)=6$.

Proof. In each case, we consider $W_{n}$ as the disjoint union of a cycle $C_{n}$ and a star $K_{1, n}$. First note that every edge in $C_{n}$ is joined to every edge in $K_{1, n}$ by a distinct third edge. It follows that the set of edge weights used on the edges of $C_{n}$ must be disjoint from the set used to weight the edges of $K_{1, n}$ by Proposition 7.1.1. Furthermore, if the edges of $K_{1, n}$ are considered in the natural cyclic order induced by adjacency to the vertices of $C_{n}$, then it is clear that consecutive "spokes" of the wheel $W_{n}$ must 
receive distinct weights. Two edge weights are necessary and sufficient to weight the spokes of $W_{n}$ if $n$ is even and three are required in $n$ is odd. One is left now only to weight the edges of $C_{n}$. By the subgraph condition given in Theorem 7.3.8, two edge weights are required for the cycle of $W_{4 k}$ and three are required for any other wheel. Each of the parameter values in the theorem follow. 


\section{Chapter 8}

\section{Conclusion}

Although the ultimate goal of showing that $\chi_{\Sigma}^{e}(G) \leq 3$ remains out of reach for the moment, we have made a good deal of progress in many of the natural generalizations. A summary of the major results contained in the thesis are found below. Table 3 on page 181 gives a summary of some conjectured and best-known upper bounds for some parameters for proper colourings derived from weightings. In each case, assume that $G$ is a nice graph where necessary, and that $D$ is a digraph. For upper bounds established in other papers the citation is given, and for new bounds proven in this thesis the page number is given. In the process of studying variations of the 1-2-3 Conjecture, ideas for future research problems have arisen; these ideas are also included below. 


\subsection{Summary and open problems}

\section{Digraphs}

The natural generalization of the 1-2-3 Conjecture for digraphs (see [15]) colours a vertex with the difference between the sum of the weights on inbound arcs and the sum of the weights on outbound arcs.

Independently of Bartnicki et al. [15], we have proven the following result:

Theorem 5.1.2. For any digraph $D, \operatorname{ch}_{\Sigma}^{e}(D) \leq 2$.

Furthermore, it is easy to characterize precisely which digraphs have $\chi_{\Sigma}^{e}(D)=1$ (those having no adjacent vertices with equal weighted degrees) and $\chi_{\Sigma}^{e}(D)=2$ (all other digraphs).

\section{Graphs with small values of $\chi_{\Sigma}^{e}(G)$}

The study of graphs for which $\chi_{\Sigma}^{e}(G) \leq 2$ is motivated by the knowledge that almost every graph has this property (see [3]). We consider the more general problem of edge weightings from a fixed set $\{a, b\}$ which properly colour vertices by sums. In Chapter 3 , such weightings were considered for trees, cycles, complete graphs, generalized theta graphs, bipartite graphs, unicyclic graphs, and infinite classes of graph products. One particular class of bipartite graphs of interest are round graphs, or graphs having no cycles of length $1,2,3(\bmod 4)$. The following notable theorems were proven:

Theorem 3.2.13. Let $d \geq 3$ and let $a, b$ be real numbers. The graph $\Theta_{\left(m_{1}, m_{2}, \ldots, m_{d}\right)}$, where $m_{1}, m_{2}, \ldots, m_{d} \in \mathbb{Z}^{+}$, is 2-weight colourable if and only if it is not of the form $\Theta_{\left(1,4 k_{2}+1, \ldots, 4 k_{d}+1\right)}$ with $k_{2}, \ldots, k_{d} \in \mathbb{Z}^{+}$. 
Theorem 3.3.7. Every round graph is $\{a, b\}$-weight colourable for any $a, b \in \mathbb{R}$ whose ratio does not reduce to a ratio of odd integers and for which ab $>0$.

The only bipartite graphs for which it is known that $\chi_{\Sigma}^{e}(G) \geq 3$ are cycles of length $1,2,3(\bmod 4)$ and generalized theta graphs $\Theta_{\left(1,4 k_{2}+1, \ldots, 4 k_{d}+1\right)}$. This prompted the following question:

Problem 3.3.9. Is it true that all bipartite graphs except $C_{4 k+2}$ and $\Theta_{\left(1,4 k_{1}+1,4 k_{2}+1, \ldots, 4 k_{d}+1\right)}$ are 2 -weight colourable?

After developing gadget graphs which can be used to force certain edge weighting properties on appropriately defined graphs, the following open problems are suggested:

Problem 8.1.1. Does there exist a graph which is uniquely $\{1,2,3\}$-weight colourable up to isomorphism? Does there exist a $\{1,2,3\}$-weight colourable graph where certain edges are always forced to receive particular weights? Moreover, does there exist a graph with either of these properties which maintains that property when it is attached in some way to another graph?

In [66], we noted that it was unknown (at the time of submission) how difficult it is to decide if a given graph admits a $\{1,2\}$-weight colouring, or more generally an $\{a, b\}$-weight colouring. As such, we posed the following problem:

Problem 8.1.2. Is it NP-complete to decide whether a given graph is 2-weight colourable?

Dudek and Wajc [41] have recently proven that it is NP-complete to determine whether or not a graph $G$ admits an $S$-weight colouring for $S=\{1,2\}$ and $S=\{0,1\}$. 
The general problem remains open, though they suggest that their methods may work for any set of two rational numbers.

\section{List variations of the 1-2-3 and 1-2 Conjectures}

Until now, no upper bound was known on $\operatorname{ch}_{\Sigma}^{e}(G)$ or $\operatorname{ch}_{\Pi}^{e}(G)$ and no upper bound for $\operatorname{ch}_{\Sigma}^{t}(G)$ was known other than the trivial bound of $\operatorname{ch}(G)$ (and hence $\Delta(G)+1$ ). By using the Combinatorial Nullstellensatz, we have been able to show that each parameter is bounded as follows:

Theorem 4.2.1. If $G$ is a nice graph with maximum degree $\Delta(G)$, then $\operatorname{ch}_{\Sigma}^{e}(G) \leq 2 \Delta(G)+1$. If $G$ is any graph, then $\operatorname{ch}_{\Sigma}^{t}(G) \leq\left\lceil\frac{2}{3} \Delta(G)\right\rceil+1$.

Theorem 4.2.9. If $G$ is a nice graph with maximum degree $\Delta(G)$, then $\operatorname{ch}_{\Pi}^{e}(G) \leq 4 \Delta(G)+2$.

A decomposition theorem with respect to edge cuts is also proven in Theorem 4.3.1, which implies improved bounds on $\mathrm{ch}_{\Sigma}^{e}(G)$ for some Cartesian products of graphs.

Theorem 4.2.1 relies on finding an orientation of a graph $G$ such that there is a subgraph $H$ of $G$ for which adding and/or subtracting the columns of $A_{D}$ or $M_{D}$ indexed by the edges of $H$ leads to lots of cancellation (see Section 4.1 for the definitions of $A_{D}, B_{D}$ and $M_{D}$ ). This allows the construction of a new matrix where no column of the original is used too many times. This subgraph used for Theorem 4.2.1 is quite simple - an induced path of length 2. It would be interesting to know if every graph $G$ has a subgraph $H$ which fills the same role but leads to improved bounds on $\operatorname{ch}_{\Sigma}^{e}(G)$ and $\operatorname{ch}_{\Sigma}^{t}(G)$. 
Alon and Tarsi [7], and many others since, have noted a connection between vertex colourings and Eulerian sub-digraphs of orientations. Let $E E(D)$ and $E O(D)$ denote the sets of Eulerian subgraphs of a digraph $D$ which contain an even and odd number of edges, respectively (recall that a subgraph $H$ of a digraph $D$ is Eulerian if $d_{H}^{-}(v)=d_{H}^{+}(v)$ for every vertex $\left.v \in V(H)\right)$.

The following theorem, presented in [80], is a generalization of a result of Alon and Tarsi in [7]; it may be useful for calculating the permanent of a particular submatrix of $B_{D}^{(k)}$ :

Theorem 8.1.3 (Schauz $[80])$. Let $G$ be a graph with vertex set $V(G)=\left\{v_{1}, \ldots, v_{n}\right\}$ and edge set $E(G)=\left\{e_{1}, \ldots e_{m}\right\}$. Let $D$ be an orientation of $G$ and for each edge $e_{i}$ let $u_{i}$ denote its head. Let $N_{D}$ be the $m \times m$ matrix $\left[c\left(u_{1}\right) c\left(u_{2}\right) \cdots c\left(u_{m}\right)\right]$, where each $c\left(u_{i}\right)$ is the column corresponding to $u_{i}$ in $B_{D}$. The permanent of $N_{D}$ is

$$
\operatorname{per} N_{D}=|E E(D)-E O(D)| \prod_{v \in V(G)} d^{+}(v) !
$$

Recall that, for a graph $G$ with $E(G)=\left\{e_{1}, \ldots, e_{m}\right\}$ and $V(G)=\left\{v_{1}, \ldots, v_{n}\right\}$ and an orientation $D$ of $G$, we defined the polynomials

$$
\begin{aligned}
P_{D}\left(x_{1}, \ldots, x_{m}\right) & =\prod_{(u, v) \in E(D)}\left(X_{v}-X_{u}\right) \\
T_{D}\left(x_{1}, \ldots, x_{l}\right) & =\prod_{(u, v) \in E(D)}\left(Y_{v}-Y_{u}\right)
\end{aligned}
$$

where $x_{i}$ is a variable associated with $e_{i}, x_{m+j}$ is associated with $v_{j}, X_{v_{j}}=\sum_{e_{i} \ni v_{j}} x_{i}$, $Y_{v_{j}}=x_{m+j}+X_{v_{j}}$ and $l=m+n$. If $|E E(D)-E O(D)|$ were to be shown to be nonzero in Theorem 8.1.3, then the monomial $\prod_{k=m+1}^{m+n} x_{k}^{d^{+}\left(v_{k}\right)}$ has nonzero coefficient. 
If $d=\max \left\{d^{+}\left(v_{k}\right): v \in V(D)\right\}$, then this would imply that the graph is $(d, 1)$-weight choosable (and hence $d$-choosable). Since this approach only uses vertex variables, it is in fact a way to bound a graph's choosability. It is not clear if a similar extension can be found that incorporates edge variables. If so, this would give a more direct approach to the List 1-2-3 Conjecture which avoids permanents all together.

Another possible approach to solving the List 1-2-3 Conjecture is a reformulation of Ryser's Formula, given in Section 4.1.

Ryser's Formula (Ryser [78]). If $A$ is an $n \times n$ square matrix, then

$$
\operatorname{per}(A)=(-1)^{n} \sum_{S \subseteq[n]}(-1)^{|S|} \prod_{i=1}^{n} \sum_{j \in S} a_{i j}
$$

Given a graph $G$ and an orientation $D$, Ryser's Formula can be reformulated for $A_{D}$ strictly in terms of graph theoretic properties. Given a set of edges $X \subseteq E(G)$, $d_{X}(v)$ denotes the degree of $v$ in the subgraph of $G$ whose vertices are the ends of $X$ and edges are $X$.

Theorem 8.1.4. Let $G$ be a graph with $m$ edges and $D$ an orientation of $G$. If $A_{D}$ is the usual weighting edge-incidence matrix as defined in Section 4.1, then

$$
\operatorname{per}\left(A_{D}\right)=\sum_{X \subseteq E(G)}\left((-1)^{m-|X|} \prod_{(u, v) \in A(D)}\left(d_{X}(v)-d_{X}(u)\right)\right)
$$

The proof is omitted, but is not difficult. The columns of $A_{D}$ are indexed by $A(D)$, and the entries in the column indexed by $a \in A(D)$ are 1 in each row corresponding to an arc incident to the head of $a,-1$ in each row corresponding to an arc incident to the head of $a$, and 0 otherwise. The summation $\sum_{j \in S} a_{i j}$ in Ryser's formula thus becomes 
$d_{X}(v)-d_{X}(u)$ for each arc $(u, v) \in A(D)$. Again, showing that the summation given for per $\left(A_{D}\right)$ in Theorem 8.1.4 is nonzero appears to be quite difficult.

Finally, we note the following conjecture on the permanent index of invertible matrices:

Conjecture 8.1.5 (Kahn [95]). For any nonsingular $n \times n$ matrix $A$ over any field, $\operatorname{pind}(A) \leq 2$.

While similar in statement, note that this is not directly related to the problem of determining whether or not $\operatorname{pind}\left(A_{D}\right) \leq 2$ for every graph $G$ (where $D$ is an arbitrary orientation of $G$ ). An examination of the definition of the entries of $A_{D}$ shows that, if $C$ is a directed cycle in $D$, then the sum of the row vectors associated with the edges of $C$ has 0 in every entry, and hence $D$ is singular. Still, the two problems are of the same flavour; a unifying theorem could conceivably prove both Kahn's conjecture and the List 1-2-3 Conjecture.

\section{Additive colourings}

The best previously known upper bound on $\chi_{\Sigma}^{v}(G)$ gave extraordinarily large values even for graphs with small maximum degree (see Theorem 2.3.8 and Corollary 2.3.10). We have shown, via a simple inductive proof, that a much better upper bound exists.

Theorem 4.4.2. For any graph $G, \operatorname{ch}_{\Sigma}^{v}(G) \leq \Delta(G)^{2}+1$.

This bound is improved for chordal graphs in Theorem 4.4.3, and the Additive Colouring Conjecture is verified for complete multipartite graphs in Theorem 4.4.4. 


\section{Colouring by sequences}

The first major result of Chapter 6 shows that for any 2-list assignment to the edges of a nice graph $G$, there exists a weighting function into these lists and an ordering of the edges so that $V(G)$ is properly coloured by sequences, where the order of elements in a vertex's colour is determined by the ordering of $E(G)$. In notation, $\operatorname{ch}_{\sigma^{*}}^{e}(G) \leq 2$ for any nice graph $G$.

Theorem 6.2.3. Let $G$ be a nice connected graph and for each $e \in E(G)$ let $L_{e}$ be a set of two real numbers. There is an ordering of $E(G)$ and values $w(e) \in L_{e}$, $e \in E(G)$, such that $w$ is a prefix distinguishing vertex colouring by sequences.

Theorem 6.2.3 generalized to total weightings, and both results generalized to multigraphs.

Using the Lovász Local Lemma, many results were proven in Section 6.3 on edge list-weightings which properly colour vertices by sequences when the ordering of $E(G)$ is arbitrary. Firstly, in light of the List 1-2-3 and List 1-2 Conjectures, we propose the following (weaker) conjectures:

Conjecture 6.3.4. If $G$ is a nice graph then $\operatorname{ch}_{\sigma}^{e}(G) \leq 3$.

Conjecture 6.3.5. For any graph $G, \operatorname{ch}_{\sigma}^{t}(G) \leq 2$.

The major results on edge weightings are as follows (recall that many have extensions to total weightings and multigraphs):

Theorem 6.3.6. Let $G$ be a graph with minimum degree $\delta(G)=\delta$ and maximum degree $\Delta(G)=\Delta$. If $\delta>\log _{3}\left(2 \Delta^{2}-2 \Delta+1\right)+2$ then $\operatorname{ch}_{\sigma}^{e}(G) \leq 3$. 
Regular graphs are of particular interest since sequences of different lengths are necessarily distinct. It follows from the proof of Theorem 6.3.6 that if $G$ is a $d$-regular graph, then $\operatorname{ch}_{\sigma}^{e}(G) \leq 3$ if $d \geq 6, \operatorname{ch}_{\sigma}^{e}(G) \leq 4$ if $d=5, \operatorname{ch}_{\sigma}^{e}(G) \leq 5$ if $d=4$, and $\operatorname{ch}_{\sigma}^{e}(G) \leq 6$ if $d=3$.

By imposing a girth condition on $G$ and considering edge $k$-weightings, we obtain a similar result for graphs with smaller minimum degree.

Theorem 6.3.11. Let $G$ be a graph with minimum degree $\delta(G)=\delta$ and maximum degree $\Delta(G)=\Delta$ and girth at least 5. If $\delta>\log _{3}\left(\Delta^{2}-\Delta+1\right)+2$ then $\chi_{\sigma}^{e}(G) \leq 3$.

Since $\chi_{m}^{e}(G) \leq 3$ if $\delta(G) \geq 1000$ by Corollary 2.2 .4 , the only graphs for which it is possible to have $\chi_{\sigma}^{e}(G) \geq 4$ (and hence $\chi_{\sigma}^{e}(G)=4$ by Theorem 2.2.3) are those with minimum degree at most 1000 and those with maximum degree exponential in terms of the minimum degree.

In Section 6.4, a sequence variation of irregularity strength was considered. Corollary 6.4 .4 states that $M_{G} \leq \mathrm{s}_{\sigma^{*}}^{e}(G) \leq \mathrm{s}_{\sigma}^{e}(G) \leq C M_{G}$, where $M_{G}=\max \left\{\left\lceil n_{i}^{1 / i}\right\rceil: 1 \leq i \leq \Delta(G)\right\}$ and $n_{i}$ denotes the number of vertices of degree $i$ in a graph $G$. Values of $C$ can be obtained by set irregularity strength (Theorem 2.6.4, see [25]) and, in the case of $d$-regular graphs, multiset irregularity strength (Theorem 2.6.3, see [5]). In Theorem 6.4.5, this value of $C$ is improved for proper colourings by sequences in $d$-regular graphs; this bound is reformulated for general graphs as follows:

Theorem 6.4.7. If $G$ is a graph with minimum degree $\delta(G)=\delta$ and maximum degree $\Delta(G)=\Delta$, then

$$
\operatorname{ls}_{\sigma}^{e}(G) \leq\left\lceil(2 e(\Delta+1)(n-\Delta))^{1 / \delta-1}\right\rceil \text {, and } \operatorname{ls}_{\sigma}^{t}(G) \leq\left\lceil(2 e(\Delta+1)(n-\Delta))^{1 / \delta}\right\rceil \text {. }
$$


As a consequence, for any $k \in \mathbb{Z}^{+}$, any graph $G$ with minimum degree $\delta(G)>c_{k} \log n$ has $\operatorname{ls}_{\sigma}^{e}(G) \leq k$, where $c_{k}$ is a constant depending only on $k$.

Corollary 6.4.8. Let $n, k \in \mathbb{Z}^{+}$. If $G$ is a graph on $n$ vertices with minimum degree $\delta(G)=\delta>\log _{k}\left(\frac{e}{2}(n+1)^{2}+1\right)+1$, then $\operatorname{ls}_{\sigma}^{e}(G) \leq k$.

The following interesting question was communicated independently by Mike Newman and Jarosław Grytczuk:

Problem 8.1.6. Let $G$ be a graph with genus $g$ and $S$ an oriented surface of genus $g$. Given a drawing of $G$ in $S$ with no crossings, what is the smallest $k$ for which there exists an edge weighting $w: E(G) \rightarrow[k]$ such that $w$ properly colours $V(G)$ by the cycles of edge weights seen by each vertex (in the order given by the drawing on $S$ )?

The 1-2-3 Conjecture would suggest that $k \leq 3$ for any graph $G$; certainly it is at most 4 due to Theorem 2.2.3.

\section{Colouring by nearly disjoint multisets and injective edge colourings}

By using an edge weighting to assign vertices multisets such that adjacent vertices have multisets that are as different as possible (that is, the weight of the edge connecting them is the only common element), a colouring problem arises with similarities to $t$-fold vertex colourings (used to study fractional colourings) and injective colourings of line graphs. We defined $\chi_{\cap}^{e}(G)$ to be the fewest number of edge weights needed to achieve such a weighting and defined $\chi_{i}^{\prime}(G)$ to be the injective chromatic index of $G$, or the injective chromatic number of its line graph. 
Sections 7.3 and 7.4 contain a number of bounds on $\chi_{n}^{e}(G)$ and $\chi_{i}^{\prime}(G)$, the majority of which are proven by using graph homomorphisms. We saw that $\chi_{n}^{e}(G)=\chi_{i}^{\prime}(G)$ if $G$ is a complete graph. On the other hand, Theorem 7.4.7 states that, for any $k$, there exists a graph with chromatic number $k$ for which $\chi_{\Gamma}^{e}(G)$ and $\chi_{i}^{\prime}(G)$ arbitrarily differ (for example, complete bipartite graphs). The values of $\chi_{n}^{e}(G)$ and $\chi_{i}^{\prime}(G)$ are determined for both of these graph classes. Bounds on $\chi_{\cap}^{e}(G)$ are also proven for 3colourable graphs, trees, Cartesian products of cycles of length $0(\bmod 4)$, and wheels. Recall that the bounds for the latter three classes of graphs are constant (Theorems 7.4.8, 7.4.9, and 7.4.10, respectively). Some experimentation suggests that this is true for all planar graphs.

Conjecture 8.1.7. There exists an integer $k$ such that $\chi_{\cap}^{e}(G) \leq k$ for any planar graph $G$.

The graph in Figure 21, found on page 177 is shown to have $\chi_{\cap}^{e}(G) \leq 8$. Via tedious case analysis, it can also be shown that $\chi_{\cap}^{e}(G)>7$. One begins by noting that $G$ consists of a "central" $K_{4}$, with three $K_{4}$ 's pasted to the edges of its outer face. By beginning with the 6 colours necessary for the central $K_{4}$, one finds that one additional edge weight is not enough to avoid a colouring conflict. Hence, if Conjecture 8.1 .7 is true, then such a $k$ must be at least 8 .

Looking forward, note that Corollaries 7.2.3 and 7.4.2 combine to give the following bounds on $\chi_{\Gamma}^{e}(G)$ and $\chi_{i}^{\prime}(G)$ :

Theorem 8.1.8. For any nice graph $G,\left(\begin{array}{c}\omega(G) \\ 2\end{array}\right) \leq \chi_{\Pi}^{e}(G) \leq \chi_{i}^{\prime}(G) \leq 2 \Delta(G)^{2}-2 \Delta(G)$.

Neither bound is best possible, unfortunately. If $G=K_{m, n}$ is a complete bipartite graph, then $\chi_{\cap}^{e}(G)=\min \{m, n\}$ by Theorem 7.4.3, and so $\chi_{\cap}^{e}(G)>\left(\begin{array}{c}\omega(G) \\ 2\end{array}\right)=1$ when 


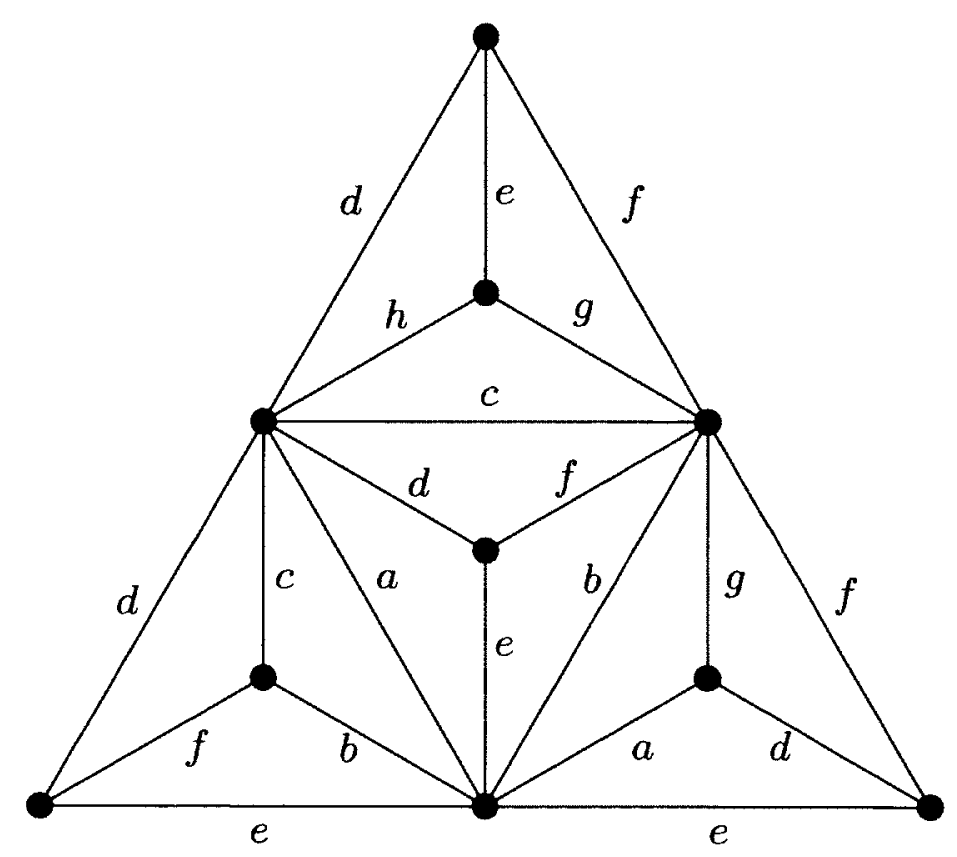

Figure 21: A planar graph with $\chi_{\cap}^{e}(G)=8$

$G$ is not a star. If $G=K_{n}$ is a complete graph, then $\chi_{\cap}^{e}(G)=\left(\begin{array}{l}n \\ 2\end{array}\right)$ by Theorem 7.4.1, which is strictly less than $2 \Delta(G)^{2}-2 \Delta(G)=2(n-1)(n-2)$ for $n \geq 3$.

Question 1. Can $\chi_{n}^{e}(G)$ can be more precisely described in terms of other graph parameter $(s)$ ?

Note the similarities between the bounds from Theorem 8.1.8 and the well known bounds on $\chi(G)$ :

$$
\begin{aligned}
\omega(G) \leq \chi(G) & \leq \Delta(G)+1 \\
C_{1} \omega(G)^{2} & \leq \chi_{\cap}^{e}(G) \leq C_{2} \Delta(G)^{2}
\end{aligned}
$$


where $C_{1}, C_{2}$ are constants. This suggests that, perhaps, $\chi_{\cap}^{e}(G)$ may be expressed as some quadratic function of $\chi(G)$. Unfortunately this is not the case, since $\chi_{n}^{e}\left(K_{m, n}\right)=\min \{m, n\}$ (and, of course, $\chi\left(K_{m, n}\right)=2$ ).

Alternately, $\chi_{\cap}^{e}(G)$ may be considered as a generalization of $\chi^{\prime}(G)$. Consider a graph $G$ which is properly edge-coloured; the subgraph of $G$ consisting of all edges with a given colour is a forest where each tree has diameter 1 (i.e. each tree is an edge) and the distance between each tree is at least 1. If $G$ is edge-weighted so that $V(G)$ is coloured by 1-intersecting multisets, then we can similarly consider the structure of a subgraph of $G$ induced by the edges with a given weight. It is not hard to see that such a subgraph is a forest, that each tree has diameter at most 2 , and the distance between any two such trees is at least 2. Conversely, any such colouring/weighting of the edges of $G$ can be seen to colour $V(G)$ by 1-intersecting multisets. This might suggest that there exist Vizing-type bounds for $\chi_{n}^{e}(G)$ in terms of $\Delta(G)^{2}$. However, we know that this is not the case, since Theorem 7.4 .3 implies that $\chi_{n}^{e}\left(K_{2, n}\right)=2$ (and, of course, $\Delta\left(K_{2, n}\right)=n$ ).

While it is not yet clear how $\chi_{\cap}^{e}(G)$ behaves, the discussion above does prompt us to suggest the following interesting colouring problem:

Problem 8.1.9. Let $c_{k}$ be an edge colouring function on a graph $G$ such that the subgraph induced by one colour is a forest of trees of diameter at most $k$, any two of which are at distance at least $k$ from one another. For a graph $G$ and an integer $k$, determine the fewest number of colours needed for such a colouring $c_{k}$ to exist. 


\section{Miscellaneous related problems}

The vast majority of the work done on proper colourings derived from weightings by sums and products focuses on real-numbered weightings. Anholcer and Cichacz have recently considered group-weightings in [12], where they define $\chi_{g}^{\Sigma}(G)$ to be the minimum $s$ such that, for any abelian group $(\Gamma,+)$ of order $s$, a graph $G$ has an edge weighting from $\Gamma$ which colours $V(G)$ by sums.

Theorem 2.2.12. If $G$ is a graph with no component having fewer than 3 vertices, then $\chi(G) \leq \chi_{g}^{\Sigma}(G) \leq \chi(G)+2$.

Since a graph is required to be able to be weighted from any finite abelian group of a given order, at least $\chi(G)$ weights are shown to be necessary. In contrast, working over the real integers when studying $\chi_{\Sigma}^{\epsilon}(G)$ allows vertex colours to be arbitrary large (subject to the degree of a vertex), and this allows one to use a constant number of weights.

Very generally speaking, weightings need only come from a semigroup (a set together with a binary operation; or, a group without an identity element or the inverse property). For example, the semigroup generated by $\{1,2,3,4,5\}$ under real number addition gives the positive integers. Of course, $\{1,2,3,4,5\}$ is not the smallest generating set for $\mathbb{Z}^{+}$; that is, of course, $\{1\}$. Clearly, a finitely generated semigroup does not always permit a edge weighting from an arbitrary generating set which is a proper vertex colouring by the semigroup's operation. The proof of the following proposition, given in Section 2.4, shows that there are finitely generated semigroups (and, in fact, monoids) for which no generating set permits a vertex colouring edge weighting. 
Proposition 2.4.7. If $G$ is a graph with $\chi(G) \geq 3$, then both $\operatorname{ch}_{\Pi}^{e}(G)$ and $\operatorname{ch}_{\Pi}^{t}(G)$ are at least 3.

In the proof, it is shown that total weighting from the set $\{-1,1\}$ for every element fails for bipartite graphs. Note that we have a finite set which generates a monoid (a group without the inverse property). Of course, since the monoid that is generated is finite, a general graph cannot possibly be properly vertex coloured from its elements. This is also the case for edge weightings which properly colour vertices by sets. Any finite set has a finite number of subsets, the set of all subsets of a finite set forms a semigroup under the union operation, and we have seen that a constant number of edge weights does not suffice for $\chi_{s}^{e}(G)$ (see Theorem 2.2.7).

This discussion prompts us to ask the following questions:

Problem 8.1.10. For a fnitely-generated infinite semigroup (or monoid) $(M, *)$, let $f(M)$ be the cardinality of the smallest subset $S_{M} \subset M$ such that, for every nice graph $G$, there exists a vertex colouring edge weighting $w: E(G) \rightarrow S_{M} . I s f(M)$ finite for every such semigroup $M$ ? If so,

1. can $S_{M}$ always be chosen so that it is itself a generating set for $M$ ?

2. what is the maximum value of $f(M)$ taken over all such $M$ ? 
Table 3: Summary of derived colouring parameter values

\begin{tabular}{|c|c|c|c|}
\hline Parameter & Conjectured upper bound & Known upper bound & Reference \\
\hline$\chi_{\Sigma}^{e}(G)$ & 3 & 5 & {$[62]$} \\
\hline$\chi_{m}^{e}(G)$ & 3 & 4 & {$[1]$} \\
\hline$\chi_{\Pi}^{e}(G)$ & 3 & 5 & {$[82]$} \\
\hline$\chi_{s}^{e}(G)$ & $\left\lceil\log _{2} \chi(G)\right\rceil+1$ & $\left\lceil\log _{2} \chi(G)\right\rceil+1(+)$ & {$[51]$} \\
\hline$\chi_{\sigma^{*}}^{e}(G)$ & 2 & 2 & pg. 127 \\
\hline \multirow[t]{2}{*}{$\chi_{\sigma}^{e}(G)$} & 3 & 4 & pg. 131 \\
\hline & & 3 if $\delta(G) \in \Omega\left(\log _{3} \Delta(G)\right)$ & pg. 133 \\
\hline$\chi_{\Sigma}^{t}(G)$ & 2 & 3 & [60] \\
\hline$\chi_{m}^{t}(G)$ & 2 & 3 & {$[60]$} \\
\hline$\chi_{\Pi}^{t}(G)$ & 2 & 3 & {$[82]$} \\
\hline$\chi_{s}^{t}(G)$ & none & $\left\lceil\log _{2} \chi(G)\right\rceil+1$ & pg. 20 \\
\hline$\chi_{\sigma^{*}}^{t}(G)$ & 2 & 2 & pg. 129 \\
\hline \multirow[t]{2}{*}{$\chi_{\sigma}^{t}(G)$} & 2 & 3 & pg. 131 \\
\hline & & 2 if $\delta(G) \in \Omega\left(\log _{2} \Delta(G)\right)$ & pg. 135 \\
\hline $\operatorname{ch}_{\Sigma}^{e}(G)$ & 3 & $2 \Delta(G)+1$ & pg. 96 \\
\hline $\operatorname{ch}_{\Sigma}^{t}(G)$ & 2 & $\left\lceil\frac{2}{3} \Delta(G)\right\rceil+1$ & pg. 96 \\
\hline $\operatorname{ch}_{\Pi}^{\epsilon}(G)$ & none $(\geq 4)$ & $4 \Delta(G)+2$ & pg. 110 \\
\hline $\operatorname{ch}_{\sigma^{*}}^{e}(G)$ & 2 & 2 & pg. 127 \\
\hline $\operatorname{ch}_{\sigma}^{e}(G)$ & 3 & 3 if $\delta(G) \in \Omega(\log \Delta(G))$ & pg. 133 \\
\hline $\operatorname{ch}_{\sigma^{*}}^{t}(G)$ & 2 & 2 & pg. 129 \\
\hline $\operatorname{ch}_{\sigma}^{t}(G)$ & 2 & 2 if $\delta(G) \in \Omega(\log \Delta(G))$ & pg. 135 \\
\hline $\operatorname{ch}_{\Sigma}^{e}(D)$ & 2 & 2 & [15], pg. 120 \\
\hline$\chi_{\Sigma}^{v}(G)$ & $\chi(G)$ & $\Delta(G)^{2}+1$ & pg. 116 \\
\hline $\operatorname{ch}_{\Sigma}^{v}(G)$ & $\operatorname{ch}(G)$ & $\Delta(G)^{2}+1$ & pg. 116 \\
\hline$\chi_{\Sigma}^{\prime t}(G)$ & none & $\frac{\Delta(G)}{2}+O(\sqrt{\Delta(G) \log \Delta(G)})$ & {$[22]$} \\
\hline$\chi_{\cap}^{e}(G)^{(0)}$ & none & $\chi_{i}^{\prime}(G)$ & 149 \\
\hline$\chi_{i}^{\prime}(G)$ & none & $2 \Delta(G)(\Delta(G)-1)$ & 150 \\
\hline
\end{tabular}

${ }^{(+)}=$holds with equality for every nice graph $G,{ }^{(0)}=$ at least $\left(\begin{array}{c}\omega(G) \\ 2\end{array}\right)($ pg. 159) 


\section{List of References}

[1] L. Addario-Berry, R. E. L. Aldred, K. Dalal, and B. A. Reed. Vertex colouring edge partitions. J. Combin. Theory Ser. B, 94(2):237-244, 2005.

[2] L. Addario-Berry, K. Dalal, C. McDiarmid, B. A. Reed, and A. Thomason. Vertex-colouring edge-weightings. Combinatorica, 27(1):1-12, 2007.

[3] L. Addario-Berry, K. Dalal, and B. A. Reed. Degree constrained subgraphs. Discrete Appl. Math., 156(7):1168-1174, 2008.

[4] M. Aigner and E. Triesch. Irregular assignments of trees and forests. SIAM J. Discrete Math., 3:439-449, 1990.

[5] M. Aigner, E. Triesch, and Z. Tuza. Irregular assignments and vertexdistinguishing edge-colorings of graphs. In Combinatorics '90 (Gaeta, 1990), volume 52 of Ann. Discrete Math., pages 1-9. North-Holland, Amsterdam, 1992.

[6] S. Akbari, M. Ghanbari, S. Jahanbekam, and M. Jamaali. List coloring of graphs having cycles of length divisible by a given number. Discrete Math., 309(3):613$614,2009$.

[7] N. Alon and M. Tarsi. Colourings and orientations of graphs. Combinatorica, 12(2):125-134, 1992.

[8] Noga Alon. Combinatorial Nullstellensatz. Combin. Probab. Comput., 8(1-2):729, 1999. Recent trends in combinatorics (Mátraháza, 1995).

[9] M. Anholcer. Product irregularity strength of graphs. Discrete Math., 309(22):6434-6439, 2009. 
[10] M. Anholcer, M. Kalkowski, and J. Przybyło. A new upper bound for the total vertex irregularity strength of graphs. Discrete Math., 309(21):6316-6317, 2009.

[11] Marcin Anholcer and Sylwia Cichacz. Group irregularity strength of connected graphs. Preprint available at http://arxiv.org/abs/1205.2569v2, 2012.

[12] Marcin Anholcer and Sylwia Cichacz. Group sum chromatic number of graphs. Preprint available at http://arxiv.org/abs/1205.2572, 2012.

[13] P.N. Balister, E. Győri, J. Lehel, and R.H. Schelp. Adjacent vertex distinguishing edge-colorings. SIAM J. Discrete Math., 21(1):237-250, 2007.

[14] Jean-Luc Baril and Olivier Togni. Neighbor-distinguishing $k$-tuple edge-colorings of graphs. Discrete Math., 309(16):5147-5157, 2009.

[15] T. Bartnicki, J. Grytczuk, and S. Niwczyk. Weight choosability of graphs. J. Graph Theory, 60(3):242-256, 2009.

[16] M. Bača, S. Jendrol', M. Miller, and J. Ryan. On irregular total labellings. Discrete Math., 307(11-12):1378-1388, 2007.

[17] Cristina Bazgan, Amel Harkat-Benhamdine, Hao Li, and Mariusz Woźniak. On the vertex-distinguishing proper edge-colorings of graphs. J. Combin. Theory Ser. B, 75(2):288-301, 1999.

[18] M. Behzad. M. Behzad, Graphs and their chromatic numbers. PhD thesis, Michigan State University, 1965.

[19] F. Bernstein. Zur theorie der trigonometrische reihen. Leipz. Ber., 60:325-328, 1908.

[20] J. A. Bondy and U. S. R. Murty. Graph theory, volume 244 of Graduate Texts in Mathematics. Springer, New York, 2008.

[21] B. Bosek, T. Bartnicki, S. Czerwiński, J. Grytczuk, G. Matecki, and W. Żelazny. Additive colorings of planar graphs. Preprint available at http://arxiv.org/abs/1202.0667v2, 2012.

[22] S. Brandt, K. Budajová, D. Rautenbach, and M. Stiebitz. Edge colouring by total labellings. Discrete Math., 310(2):199-205, 2010. 
[23] Stephan Brandt, Jozef Miškuf, and Dieter Rautenbach. Edge irregular total labellings for graphs of linear size. Discrete Math., 309(12):3786-3792, 2009.

[24] Stephan Brandt, Jozef Miškuf, Dieter Rautenbach, Friedrich Regen, and Imre Z. Ruzsa. Edge-injective and edge-surjective vertex labellings. SIAM J. Discrete Math., 24(2):666-683, 2010.

[25] A. C. Burris and R. H. Schelp. Vertex-distinguishing proper edge-colourings. J. Graph Theory, 26(2):73-82, 1997.

[26] M. I. Buršteinn. Every 4-valent graph has an acyclic 5-coloring. Soobshch. Akad. Nauk Gruzin. SSR, 93(1):21-24, 1979.

[27] Ivan Chajda and Helmut Länger. Quotients and homomorphisms of relational systems. Acta Univ. Palack. Olomuc. Fac. Rerum Natur. Math., 49(2):37-47, 2010.

[28] Gerard J. Chang, Changhong Lu, Jiaojiao Wu, and Qinglin Yu. Vertex-coloring edge-weightings of graphs. Taiwanese J. Math., 15(4):1807-1813, 2011.

[29] Gary Chartrand, Michael S. Jacobson, Jenő Lehel, Ortrud R. Oellermann, Sergio Ruiz, and Farrokh Saba. Irregular networks. Congr. Numer., 64:197-210, 1988. 250th Anniversary Conference on Graph Theory (Fort Wayne, IN, 1986).

[30] Gary Chartrand, Linda Lesniak, Donald W. VanderJagt, and Ping Zhang. Recognizable colorings of graphs. Discuss. Math. Graph Theory, 28(1):35-57, 2008.

[31] Gary Chartrand, Futaba Okamoto, Craig W. Rasmussen, and Ping Zhang. The set chromatic number of a graph. Discuss. Math. Graph Theory, 29(3):545-561, 2009 .

[32] Gary Chartrand, Futaba Okamoto, Ebrahim Salehi, and Ping Zhang. The multiset chromatic number of a graph. Math. Bohem., 134(2):191-209, 2009.

[33] Gary Chartrand, Futaba Okamoto, and Ping Zhang. Neighbor-distinguishing vertex colorings of graphs. J. Combin. Math. Combin. Comput., 74:223-251, 2010 .

[34] Gary Chartrand, Futaba Okamoto, and Ping Zhang. The sigma chromatic number of a graph. Graphs Combin., 26(6):755-773, 2010. 
[35] Tom Coker and Karen Johannson. The adjacent vertex distinguishing total chromatic number. Preprint available at http://arxiv.org/abs/1009.1785, 2010 .

[36] S. Czerwiński, J. Grytczuk, and W. Żelazny. Lucky labelings of graphs. Inform. Process. Lett., 109(18):1078-1081, 2009.

[37] Akbar Davoodi and Behnaz Omoomi. On the 1-2-3-conjecture. Preprint available at http://arxiv.org/abs/1205.3266, 2012.

[38] Reinhard Diestel. Graph theory, volume 173 of Graduate Texts in Mathematics. Springer, Heidelberg, fourth edition, 2010.

[39] Alain Doyon, Geňa Hahn, and André Raspaud. Some bounds on the injective chromatic number of graphs. Discrete Math., 310(3):585-590, 2010.

[40] Y. H. Duan, H. L. Lu, and Q. Yu. $l$-factors and adjacent vertexdistinguishing edge-weighting. Front. Math. China (submitted). Preprint available at http://arxiv.org/abs/1007.1295, 2010.

[41] Andrzej Dudek and David Wajc. On the complexity of vertex-coloring edgeweightings. Discrete Math. Theor. Comput. Sci., 13(3):45-50, 2011.

[42] N. Eaton. On two short proofs about list coloring. Online; accessed 20-January2011, 2003. http://www.math.uri.edu/ eaton/TalkUriDct03P2.pdf.

[43] P. Erdös and L. Lovász. Problems and results on 3-chromatic hypergraphs and some related questions. In Infinite and finite sets (Colloq., Keszthely, 1973; dedicated to P. Erdös on his 60th birthday), Vol. II, pages 609-627. Colloq. Math. Soc. János Bolyai, Vol. 10. North-Holland, Amsterdam, 1975.

[44] Paul Erdős, Arthur L. Rubin, and Herbert Taylor. Choosability in graphs. In Proceedings of the West Coast Conference on Combinatorics, Graph Theory and Computing (Humboldt State Univ., Arcata, Calif., 1979), Congress. Numer., XXVI, pages 125-157, Winnipeg, Man., 1980. Utilitas Math.

[45] Louis Esperet, Sylvain Gravier, Mickaël Montassier, Pascal Ocehm, and Aline Parreau. Locally identifying coloring of graphs. Preprint available at http://arxiv.org/abs/1010.5624v2, 2010. 
[46] Florent Foucaud, Iiro Honkala, Tero Laihonen, Aline Parreau, and Guillem Perarnau. Locally identifying colourings for graphs with given maximum degree. Discrete Mathematics, 312(10):1832 - 1837, 2012.

[47] O. Frank, F. Harary, and M. Plantholt. The line-distinguishing chromatic number of a graph. Ars Combin., 14:241-252, 1982.

[48] Joseph A. Gallian. A dynamic survey of graph labeling. Electron. J. Combin., 5:Dynamic Survey 6, 43 pp. (electronic), 1998.

[49] Branko Grünbaum. Acyclic colorings of planar graphs. Israel J. Math., 14:390 408, 1973.

[50] E. Győri, M. Horňák, C. Palmer, and M. Woźniak. General neighbourdistinguishing index of a graph. Discrete Math., 308(5-6):827-831, 2008.

[51] E. Györi and C. Palmer. A new type of edge-derived vertex coloring. Discrete Math., 309(22):6344-6352, 2009.

[52] Geňa Hahn, Jan Kratochvíl, Jozef Širán̆, and Dominique Sotteau. On the injective chromatic number of graphs. Discrete Math., 256(1-2):179-192, 2002.

[53] Frank Harary and Michael Plantholt. Graphs with the line-distinguishing chromatic number equal to the usual one. Utilitas Math., 23:201-207, 1983.

[54] Frank Harary and Michael Plantholt. The point-distinguishing chromatic index. In Graphs and applications (Boulder, Colo., 1982), Wiley-Intersci. Publ., pages 147-162. Wiley, New York, 1985.

[55] H. Hatami. $\delta+300$ is a bound on the adjacent vertex distinguishing edge chromatic number. J. Combin. Theory Ser. B, 95(2):246-256, 2005.

[56] Mirko Horñák and Mariusz Woźniak. On neighbour-distinguishing colourings from lists. In Fifth Cracow Conference on Graph Theory USTRON '06, volume 24 of Electron. Notes Discrete Math., pages 295-297 (electronic). Elsevier, Amsterdam, 2006. Extended abstract. Paper available at http://www.ii.uj.edu.pl/preMD/MD_29.pdf. 
[57] Sogol Jahanbekam and Douglas B. West. The 1,2-conjecture for graphs with relatively small chromatic number. Preprint available at ww. math.uiuc. edu/ west/pubs/12conj.ps, 2012.

[58] Tommy R. Jensen and Bjarne Toft. Graph coloring problems. Wiley-Interscience Series in Discrete Mathematics and Optimization. John Wiley \& Sons Inc., New York, 1995. A Wiley-Interscience Publication.

[59] A. Johnson and F. C. Holroyd. Overlap colourings of graphs. Congr. Numer., 113:221-230, 1996. Festschrift for C. St. J. A. Nash-Williams.

[60] M. Kalkowski. A note on the 1,2-conjecture. Preprint, private communication, 2009.

[61] M. Kalkowski, M. Karoniski, and F. Pfender. Vertex colouring edge weightings with integer weights at most 6. Rostock. Math. Kolloq., 64:39-43, 2009.

[62] M. Kalkowski, M. Karoński, and F. Pfender. Vertex-coloring edge-weightings: towards the 1-2-3-conjecture. J. Combin. Theory Ser. B, 100(3):347-349, 2010.

[63] M. Kalkowski, M. Karoński, and F. Pfender. A new upper bound for the irregularity strength of graphs. SIAM J. Discrete Math., 25(3):1319-1321, 2011.

[64] M. Karoński, T. Łuczak, and A. Thomason. Edge weights and vertex colours. J. Combin. Theory Ser. B, 91(1):151-157, 2004.

[65] Mahdad Khatirinejad, Reza Naserasr, Mike Newman, Ben Seamone, and Brett Stevens. Digraphs are 2-weight choosable. Electron. J. Combin., 18(1):Paper 21, 4,2011 .

[66] Mahdad Khatirinejad, Reza Naserasr, Mike Newman, Ben Seamone, and Brett Stevens. Vertex-colouring edge-weightings with two edge weights. Discrete Mathematics $\&$ Theoretical Computer Science, 14(1), 2012.

[67] J. Lehel. Facts and quests on degree irregular assignments. In Graph theory, combinatorics, and applications. Vol. 2 (Kalamazoo, MI, 1988), Wiley-Intersci. Publ., pages 765-781. Wiley, New York, 1991.

[68] L. Liu, J. Wang, and Z. Zhang. Adjacent strong edge coloring of graphs. Appl. Math. Lett., 15(5):623-626, 2002. 
[69] H. Lu, X. Yang, and Q. Yu. On vertex-coloring edge-weighting of graphs. Front. Math. China, 4(2):325-334, 2009.

[70] H. Lu, Q. Yu, and C. Zhang. Vertex-coloring 2-edge-weighting of graphs. European J. Combin., 32(1):21-27, 2011.

[71] Henryk Minc. Permanents, volume 9999 of Encyclopedia of Mathematics and its Applications. Addison-Wesley Publishing Co., Reading, Mass., 1978. With a foreword by Marvin Marcus, Encyclopedia of Mathematics and its Applications, Vol. 6 .

[72] Michael Molloy and Bruce Reed. A bound on the total chromatic number. Combinatorica, 18(2):241-280, 1998.

[73] T. Nierhoff. A tight bound on the irregularity strength of graphs. SIAM J. Discrete Math., 13(3):313-323, 2000.

[74] O. Pikhurko. Characterization of product anti-magic graphs of large order. Graphs Combin., 23(6):681-689, 2007.

[75] Jakub Przybyło. A note on neighbour-distinguishing regular graphs totalweighting. Electron. J. Combin., 15(1):Note 35, 5, 2008.

[76] Jakub Przybyło and Mariusz Woźniak. On a 1,2 conjecture. Discrete Math. Theor. Comput. Sci., 12(1):101-108, 2010.

[77] Jakub Przybyło and Mariusz Woźniak. Total weight choosability of graphs. Electron. J. Combin., 18(1):Paper 112, 11, 2011.

[78] Herbert John Ryser. Combinatorial mathematics. The Carus Mathematical Monographs, No. 14. Published by The Mathematical Association of America, 1963.

[79] N. Zagaglia Salvi. A note on the line-distinguishing chromatic number and the chromatic index of a graph. J. Graph Theory, 17(5):589-591, 1993.

[80] Uwe Schauz. Colorings and orientations of matrices and graphs. Electron. J. Combin., 13(1):Research Paper 61, 12 pp. (electronic), 2006. 
[81] I. Schur. Über endliche Gruppen und Hermitesche Formen. Math. Z., 1(2-3):184207, 1918.

[82] J. Skowronek-Kaziów. 1,2 conjecture - the multiplicative version. Inform. Process. Lett., 107(3-4):93-95, 2008.

[83] Joel Spencer. Asymptotic lower bounds for Ramsey functions. Discrete Math., 20(1):69-76, 1977/78.

[84] Saul Stahl. $n$-tuple colorings and associated graphs. J. Combinatorial Theory Ser. B, 20(2):185-203, 1976.

[85] C. Thomassen. Every planar graph is 5-choosable. J. Combin. Theory Ser. B, 62(1):180-181, 1994.

[86] L. G. Valiant. The complexity of computing the permanent. Theoret. Comput. Sci., 8(2):189-201, 1979.

[87] Satish Varagani, V. Ch. Venkaiah, Kishore Yadav, and Kishore Kothapalli. Acyclic vertex coloring of graphs of maximum degree six. In LAGOS'09-V Latin-American Algorithms, Graphs and Optimization Symposium, volume 35 of Electron. Notes Discrete Math., pages 177-182. Elsevier Sci. B. V., Amsterdam, 2009.

[88] V. G. Vizing. Some unsolved problems in graph theory. Uspehi Mat. Nauk, 23(6 (144)):117-134, 1968.

[89] V. G. Vizing. Coloring the vertices of a graph in prescribed colors. Diskret. Analiz, (29 Metody Diskret. Anal. v Teorii Kodov i Shem):3-10, 101, 1976.

[90] T. Wang and Q. Yu. On vertex-coloring 13-edge-weighting. Front. Math. China, 3(4):581-587, 2008.

[91] D. West. Irregularity strength of graphs and digraphs. Online; accessed 11-April2011. Available at http://www.math.uiuc.edu/ west/regs/irreg.html.

[92] T. Wong and X. Zhu. Total weight choosability of graphs. J. Graph Theory, 66(3):198-212, 2011. 
[93] Tsai-Lien Wong, Daqing Yang, and Xuding Zhu. List total weighting of graphs. In Fete of combinatorics and computer science, volume 20 of Bolyai Soc. Math. Stud., pages 337-353. János Bolyai Math. Soc., Budapest, 2010.

[94] Kishore Yadav, Satish Varagani, Kishore Kothapalli, and V. Ch. Venkaiah. Acyclic vertex coloring of graphs of maximum degree 5. Discrete Math., 311(5):342-348, 2011.

[95] Yang Yu. The permanent rank of a matrix. J. Combin. Theory Ser. A, 85(2):237242, 1999.

[96] Zhongfu Zhang, Xiang'en Chen, Jingwen Li, Bing Yao, Xinzhong Lu, and Jianfang Wang. On adjacent-vertex-distinguishing total coloring of graphs. Sci. China Ser. A, 48(3):289-299, 2005. 